\title{
Theory and Numerics for Shape Optimization in Superconductivity
}

\author{
Dissertation \\ zur Erlangung des Doktorgrades \\ der Mathematisch-Naturwissenschaftlichen Fakultäten \\ der Georg-August-Universität zu Göttingen
}

vorgelegt von

Harald Heese

aus Kassel

Göttingen 2006 
Rerefent:

Prof. Dr. R. Kreß

Korreferent:

Prof. Dr. R. Potthast

Tag der mündlichen Prüfung: 21. 07. 2006 
Es ist nicht genug zu wissen, man muss auch anwenden.

Goethe, Wilhelm Meisters Wanderjahre 



\begin{abstract}
We consider a mathematical model for a thin superconducting film which is magnetically shielded by permanent magnets in order to improve the current carrying capability of the film. In a first part we study the behaviour of the magnetic field of the combined system, which is characterized via a boundary value problem for Laplace's equation for the quasi-scalar magnetic potential. In a second part we formulate and analyze a related geometric optimization problem that can be interpreted as a homogenization of the current distribution in the superconducting film by means of shape optimization for the magnet boundaries.

We present a uniqueness and existence analysis for the boundary value problem based on boundary integral equations. The theoretical studies are complemented by a numerical approximation scheme for the potential, for which we prove exponential convergence rates under appropriate smoothness assumptions on the geometry. As central result for the geometric optimization problem we prove the differentiable dependence of the current distribution on the geometry, which also leads to an abstract existence result. Based on the differentiability result we derive two numerical schemes to realize the geometric optimization problem iteratively. The first approach relies on explicit parametrizations for the boundaries leading to a steepest descent scheme. The second approach uses level set methods which are based on an implicit boundary representation. The feasibility of both approaches is shown in a variety of examples.
\end{abstract}





\section{Zusammenfassung}

Wir betrachten ein mathematisches Modell eines dünnen, supraleitenden Films, der durch Permanentmagneten magnetisch abgeschirmt ist, um die Stromtragfähigkeit des Films zu erhöhen. In einem ersten Teil studieren wir das Verhalten des magnetischen Felds in dem kombinierten System, welches durch ein Randwertproblem zur Laplacegleichung für das quasi-skalare magnetische Potential charakterisiert wird. In einem zweiten Teil formulieren und analysieren wir ein verwandtes geometrisches Optimierungsproblem. Dieses kann als Homogenisierung der Stromverteilung des supraleitenden Films durch Formoptimierung der Ränder der Magneten interpretiert werden.

Im ersten Teil stellen wir eine auf Randintegralgleichungen basierende Lösungstheorie für das Randwertproblem vor. Die theoretischen Untersuchungen werden durch ein numerisches Verfahren ergänzt, für welches wir unter entsprechenden Glattheitsannahmen an die Geometrie exponentielle Konvergenzraten beweisen. Der Nachweis der differenzierbaren Abhängigkeit der Stromverteilung von der Geometrie bildet das zentrale Resultat des zweiten Teils, welches auch als Grundlage für ein abstraktes Existenzresultat für das Optimierungsproblem dient. Zudem bildet die Differenzierbarkeit den Ausgangspunkt für zwei numerische Verfahren zur Umsetzung des geometrischen Optimierungsproblems. Das erste Verfahren beruht auf der Kenntnis von expliziten Randparametrisierungen und führt zu einem Verfahren des Steilsten Abstiegs. Der zweite Ansatz benutzt Level Set Methoden, welche auf einer impliziten Randdarstellung basieren. Beide Verfahren werden an einer Reihe von Beispielen illustriert. 



\section{Acknowledgements}

Finally, I would like to thank those that have supported me throughout my studies. First of all, I thank my supervisor, Prof. Dr. Rainer Kreß, for introducing me to the topic of my thesis and for the valuable discussions. He has been ready with helpful suggestions when they were needed, allowing at the same time as much room as possible for my own development. Furthermore, my thanks go to Prof. Dr. Roland Potthast for acting as the second referee.

I would also like to thank my office mates Stefan Langer and Pedro Serranho for proof-reading parts of this thesis and for the fruitful years together. My thanks are extended to the Deutsche Forschungsgemeinschaft and the Graduiertenkolleg "Identifikation in mathematischen Modellen" for the financial support, and for the opportunity to come into contact with an even greater variety of fields in mathematics.

Above all, my deep thanks go to Ulrike Stubben for keeping up with me these days, and for being a constant resource for understanding, rest and cheerfulness. 



\section{Contents}

$\begin{array}{lll}0 & \text { Introduction } & 13\end{array}$

1 Potential theory $\quad 23$

1.1 Harmonic functions . . . . . . . . . . . . . . . . . . . 23

1.2 Layer potentials . . . . . . . . . . . . . . . . . . . . 28

1.3 An invertible integral operator . . . . . . . . . . . . . . . . 38

2 A Boundary Value Problem 41

2.1 Statement of the Problem . . . . . . . . . . . . . . . . 41

2.2 Uniqueness . . . . . . . . . . . . . . . . . . . . . . . 42

2.3 Existence . . . . . . . . . . . . . . . . . . . . . 44

2.4 Properties of the solution to the BVP . . . . . . . . . . . . 52

3 A Geometric Optimization Problem $\quad 63$

3.1 Statement of the Problem . . . . . . . . . . . . . . . . 63

3.2 Properties of the GOP . . . . . . . . . . . . . . . . . . 68

3.3 Fréchet differentiability . . . . . . . . . . . . . . . . 75

3.4 Domain sensitivity results for the BVP . . . . . . . . . . 86

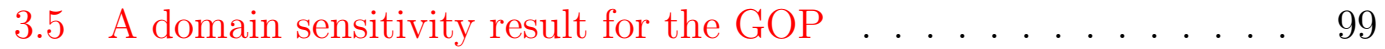

4 Numerical treatment $\quad 103$

4.1 An approximation scheme for the BVP . . . . . . . . . . . . . 104

4.2 A Steepest Descent Approach for the GOP . . . . . . . . . . . . . 110

4.3 A Level Set Approach for the GOP . . . . . . . . . . . . . . . 122

$\begin{array}{llr}5 & \text { Numerical examples } & 151\end{array}$

5.1 The Boundary Value Problem . . . . . . . . . . . . . . . . 151

5.2 The GOP using the Steepest Descent Approach . . . . . . . . . 158

5.3 The GOP using the Level Set Approach . . . . . . . . . . . . . . 173

6 Discussion and Outlook 181

6.1 Discussion . . . . . . . . . . . . . . . . . . 181

6.2 Outlook ........................ 186 


\section{Chapter 0}

\section{Introduction}

Laplace's equation is one of the most fascinating partial differential equations of mathematical physics. Its widespread occurrence in electrostatics, magnetostatics, steady state heat conduction, incompressible fluid flow, gravitation and elasticity gives evidence for its importance and versatility. Its simple nature makes it the ideal candidate to serve as a model for involved elliptic PDEs. Named after its discoverer Pierre-Simon Laplace (1749 - 1827) it has been an object of constant interest for many mathematicians such as Courant and Hilbert [19]. In its long standing history, a vast amount of methods have been developed to solve a variety of boundary value problems that can be associated with Laplace's equation, such as Dirichlet's problems or transmission problems. In a Dirichlet problem one seeks a real-valued function $u$ satisfying

$$
\left.\Delta u\right|_{D}=0,\left.\quad u\right|_{\partial D}=g
$$

for some function $g$ defined on the boundary $\partial D$ of a bounded domain. In the latter case of a transmission problem one tries to find a function $u$ that satisfies

$$
\left.\Delta u\right|_{\mathbb{R}^{2} \backslash \partial D}=0, \quad u_{+}-u_{-}=0, \quad \frac{\partial u_{+}}{\partial \nu}-a \frac{\partial u_{-}}{\partial \nu}=0
$$

for some positive constant $a \neq 1$. In the first part of the work at hand we will focus on a boundary value problem which combines the two cases described above in the sense that on some parts of the boundary we impose Dirichlet's condition whereas on others we require a possible solution to satisfy transmission conditions. For the sake of completeness we mention here that in the literature one can find also several other boundary conditions for Laplace's equation such as Neumann conditions, or impedance conditions. We refer to [48] and the references therein for a more comprehensive study on boundary value problems to Laplace's equation.

In the scientific history of boundary value problems to Laplace's equation not only the boundary conditions have played a major role, but also the boundary 
itself has been an object of interest. So, extremely specialized methods and solution formulae have been developed for circular, spherical or cylindrical objects. On the other hand the analysis has been relaxed to domains with corners or Lipschitz domains. Also unbounded surfaces, either flat or rough, have been studied extensively. Detailed descriptions of these fields can be found in [25, 74].

However, Laplace's equation does not only appear in the context of mathematical physics, it also emerges naturally in complex analysis. So, the real and imaginary part of a holomorphic function are solutions to Laplace's equation due to the Cauchy-Riemann equations. Furthermore, Cauchy's integral formula renders a beautiful way to represent holomorphic functions by an integral expression over a closed curve. Starting out from this result, it is easy to see that, in cases where the curve along which the integration is performed is not closed but an open curve, the integral expression also defines a solution to Laplace's equation in the exterior of the arc. In this way a natural construction arises that allows to study phenomena from mathematical physics that are defined in the exterior of an open curve and which can be described appropriately using Laplace's equation.

The boundary value problem, which we will consider in the second chapter will incorporate two of the described boundary structures, namely closed domains with $C^{2}$-smooth boundaries and open curves.

As manifold as the areas, boundary conditions and geometric assumptions are, that have been subject to mathematical research, as broad have been the approaches to do the analysis for these problems. Driven by the desired applicability and the mathematical fashion of the time, layer representations, series expansions or finite element methods have been developed among others (see [24, 47]). In our description we will follow the classical approach and seek solutions via layer representations and integral equation methods.

In our case the work on Laplace's equation has been motivated from a problem in magnetostatics from theoretical physics. In [30], Genenko et al. have considered a macroscopic model for the Meissner state of a thin superconducting film in a magnetic environment, which we have picked up for our considerations. In the following we will describe the modelling process that finally leads to the boundary value problem for which we will do the analysis in the first part of this work.

A priori, the model of a thin superconducting film in a magnetic environment is three-dimensional. It consists of a thin volume sample with thickness $d<<W$, where $W$ is the width, and an arbitrary finite number of permanent magnets. Both the superconducting film and the magnets are extended infinitely long in $x_{3}$-direction and are assumed to have a constant cross-section in the $x_{1} x_{2}$-plane. All physical constants are assumed to be independent of the $x_{3}$-coordinate, and the (unknown) magnetic field $\mathbf{B}$ is homogeneous in that direction. Furthermore, the superconducting film carries a transport current of total strength $I$ which is distributed over the film according to some unknown current density distribution $j: \mathbb{R}^{3} \rightarrow \mathbb{R}^{3}$. Figure 1 illustrates the three-dimensional situation decribed above for a geometric setup as it appears in experimental physics (see [41, 43]). 


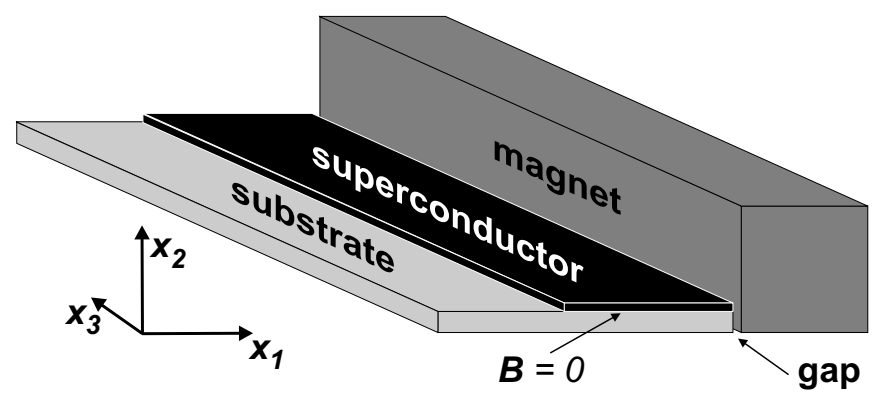

Figure 1: Geometric setup in three dimensions

In this framework we are interested in deducing the magnetic field $\mathbf{B}$ and the current distribution $j$. The model is reduced to a two-dimensional problem by considering the cross-section in the $x_{1} x_{2}$-plane and the corresponding quasi-scalar magnetic vector-potential

$$
A\left(x, x_{3}\right):=(0,0, u(x)), \quad x \in \mathbb{R}^{2},
$$

for some twice continuously differentiable function $u$. Defining

$$
\mathbf{B}:=\operatorname{curl} A
$$

we obtain that $\mathbf{B}$ satisfies the fundamental equations

$$
\begin{aligned}
\operatorname{div} \mathbf{B} & =0, \\
\operatorname{curl} \mathbf{B} & =(0,0,-\Delta u)=-\mu_{0} j
\end{aligned}
$$

of magnetostatics for a non-zero constant $\mu_{0}$ and the unknown current density distribution $j: \mathbb{R}^{3} \rightarrow \mathbb{R}^{3}$, i.e. we can think of $\mathbf{B}$ as a magnetic field. The current density $j$ is thought of as the magnetic analogon to the charge density, and it is only non-zero where the medium is penetrated by an electric current. Consequently, it has to vanish inside the magnets and in the exterior domain and may support electric sources only inside the superconducting film or on the surface of the magnets. We see from the above that the scalar magnetic potential $u$ is a solution to Laplace's equation in the interior and exterior of the magnets apart from the superconducting film. The surface of the magnets may be regarded as an interface between two materials with different magnetic permeabilities $\mu_{1}$ and $\mu_{2}$. At such interfaces $S$ between two media $V_{1}$ and $V_{2}$ the corresponding magnetic fields $\mathbf{B}_{1}$ and $\mathbf{B}_{2}$ are known to behave like

$$
\begin{aligned}
\mathbf{B}_{1} \cdot n_{S} & =\mathbf{B}_{2} \cdot n_{S}, \\
\frac{1}{\mu_{1}} \mathbf{B}_{1} \times n_{S} & =\frac{1}{\mu_{2}} \mathbf{B}_{2} \times n_{S},
\end{aligned}
$$

where $n_{S}$ denotes the surface normal to $S$. From the identity

$$
\mathbf{B}=\left(\frac{\partial u}{\partial x_{2}},-\frac{\partial u}{\partial x_{1}}, 0\right)^{T}
$$


and the fact that the surface normal to $S$ is of the form $n_{S}=\left(n_{1}, n_{2}, 0\right)^{T}$ due to the constant cross-section with respect to the $x_{3}$-direction, we can derive corresponding conditions that have to be imposed on $u$. These are, in particular,

$$
\begin{aligned}
u_{+} & =u_{-}, \\
\frac{1}{\mu_{+}} \frac{\partial u_{+}}{\partial \nu} & =\frac{1}{\mu_{-}} \frac{\partial u_{-}}{\partial \nu},
\end{aligned}
$$

where $\nu=\left(n_{1}, n_{2}\right)$ is the projection of $n_{S}$ onto the $x_{1} x_{2}$-plane which we identify with $\mathbb{R}^{2}$, and where the '+'- and '-'-signs signify whether the surface of the magnet is approached from direction $+\nu$ or $-\nu$.

In terms of theoretical physics the Meissner state of a superconducting medium $V$ is characterized by

$$
\mathbf{B}=0 \quad \text { in } V
$$

which particularly implies

$$
\mathbf{B} \cdot n_{\partial V}=0 \quad \text { on } \partial V
$$

where $\partial V$ is the surface of the superconducting medium $V$ and $n_{\partial V}$ is the surface normal. Using the relation (1) between $\mathbf{B}$ and $u$ and the form of the surface normal we can translate (2) into the condition

$$
u=\text { const } \quad \text { on } \partial V \text {, }
$$

that has to be imposed on $u$. As the potential corresponding to a certain magnetic field $\mathbf{B}$ is determined only up to a constant, we may even assume that

$$
u=0 \quad \text { on } \partial V \text {. }
$$

Interestingly, the above model enforces some constraints on the macroscopic behaviour of the magnetic field $\mathbf{B}$. Although we think of $\mathbf{B}$ as a continuously varying vector field, there exists a characteristic quantity $\lambda$ called penetration depth, according to which the magnetic field $\mathbf{B}$ is homogeneous on scales smaller than $\lambda$. The thin superconducting films that have been considered both in experiments (see [43]) and in the physical model (see [30]) satisfy the assumption $d \leq \lambda$ for the thickness of the film. Consequently, we may assume that the magnetic field $\mathbf{B}$ does not vary over the thickness of the film. This then justifies the modelling of the formally two-dimensional cross-section of the superconducting film as a one-dimensional open curve $\Gamma$. The conditions imposed on $u$ in the above are unaffected by this modelling assumption as they were formulated either for the exterior of the superconducting film or in terms of the surface $\partial V$, which now coincides with the open curve $\Gamma$.

The carrying of a non-zero transport current may be interpreted from the point of view of Biot-Savart's law

$$
\mathbf{B}(x)=\frac{\mu}{4 \pi} \iiint_{V} \frac{j(y) \times(x-y)}{|x-y|^{3}} d y .
$$


Using the assumed homogeneity with respect to the $x_{3}$-direction, we may obtain an area integral over the cross-section from the three-dimensional volume integral given in (3) with an asymptotic behaviour of $|x|^{-1}$ for $|x| \rightarrow \infty$. This implies that the potential $u$ corresponding to the magnetic field $\mathbf{B}$ which is generated by the current density $j$ is allowed to grow at a logarithmic rate, and thereby motivates the choice of the condition

$$
u(x)-\frac{I}{2 \pi|\Gamma|} \int_{\Gamma} \ln \frac{1}{|x-y|} d s(y)=\mathcal{O}(1), \quad|x| \rightarrow \infty,
$$

to be imposed on $u$. It will be the issue of Section 2.4 to verify that (4) indeed yields a total transport current $I$ in the sense of Ampère's law.

From an applicational viewpoint it is often not satisfactory to stop once an understanding of a certain model has been achieved. The aim is rather to influence or control certain aspects or quantities of the model in order to achieve a better behaviour of the model with respect to some goal. These ideas have also been present in the field of applied mathematics for a long time. Research in these areas is known generally under the name of optimization or control theory, which in its classical form mainly deals with optimizing the value of an objective functional that depends on one or several scalar parameters, which are furthermore subject to some linear or non-linear constraints (see, for example, [75] for an introduction). In the last thirty years a special branch called shape optimization has developed within the field of optimization, which concerns itself with problems where the parameter is a geometric quantity such as a domain in $n$-space or some characteristic of such a domain. In problems of this type the connection between the parameter and the objective cannot always be described through a 'simple' functional dependence, but only via the solution of a partial differential equation which then is regarded as dependent on the domain as parameter. Let us mention in this context the books by Pironneau [57], and Sokolowski and Zolesio [70], as well as the more recent monographs by Delfour and Zolesio [22] or Haslinger and Mäkinen [33] for further reference on shape optimization.

Again, various strategies have been developed to treat this type of problems which are in almost every case motivated by the nature of the parameter and the objective. Focussing on optimization with respect to domains and their boundary shape we may coarsely distinguish between two groups of approaches, which differ in their perspective of the domain boundary. On the one side we find the group of methods relying on explicit boundary representation. The methods of this group assume that the boundary of the domain is given explicitly in terms of parametrizations or in the case of polygons in terms of its corners. On the other side there are the methods relying on implicit boundary representation, such as so-called level set methods, that have become extremely popular within the last decade. In this approach the boundary is given as the 0-level set of a globallydefined, real-valued function $\phi$ using the convention that a point $x$ is inside the 


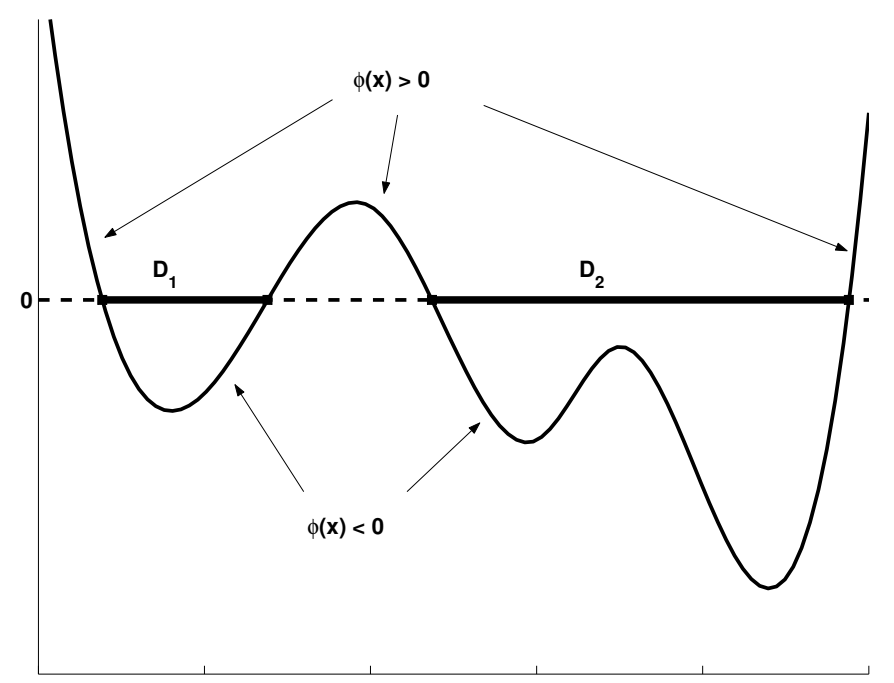

Figure 2: One-dimensional example of a level set function

domain $D$ if $\phi(x)<0$, and it is outside the domain $D$ if $\phi(x)>0$. Figure 2 gives a one-dimensional illustration for such a level set function $\phi$, where the domain $D$ consists of two connected components $D_{1}$ and $D_{2}$.

Common to both of these groups are the tools from general optimization theory with which the related problem is attacked. Starting from an initial guess the objective yields a 'direction' or an 'update' which improves the behaviour of the problem with respect to that same objective. In the first group this is interpreted as a simple iterative update process

$$
z_{n+1}:=z_{n}+h_{n}, \quad n \in \mathbb{N} \cup\{0\},
$$

where $z_{n}$ is the explicit boundary representation of the iterate $\partial D_{n}$, and $h_{n}$ is the update. In the latter group the interpretation is given by an evolution equation of the form

$$
\frac{\partial \phi(x, t)}{\partial t}+\langle V(x, t), \operatorname{grad} \phi(x, t)\rangle=0
$$

in artificial time, where $V$ is a driving vector field obtained from the objective in some appropriate way, advecting $\phi$ towards the optimum.

We will realize and compare both kinds of approaches in this work by applying them to a Geometric Optimization Problem that is derived from the macroscopic model for the Meissner state of a thin superconducting film in a magnetic environment introduced above in the following way.

The Meissner state of superconducting medium is very fragile with respect to mainly two quantities. On the one hand it is limited to comparatively low temperatures (either $<-270^{\circ} \mathrm{C}$ for pure copper or $<-150^{\circ} \mathrm{C}$ for so-called hightemperature YBCO-superconductors), on the other hand it can be maintained 
only for small magnetic fields B. In the model for the thin film described above it was assumed that an electric current penetrates the superconducting film. This current generates a magnetic field in the exterior of the film, which will eventually cause the breakdown of the Meissner state if the total current $I$ is too large. From the perspective of Ampère's law, the current $I$ is distributed over the film according to

$$
I=\iint j d x
$$

where $j$ is the current density which may not be homogeneous. Furthermore, there are two characteristic quantities, the Ginzburgh-Landau depairing current $j_{0}$ and the critical value of first flux entry $H^{*}$, that govern a possible breakdown. While $j_{0}$ is a local and microscopic quantity that gives an upper bound for the current density above which the film immediately becomes normally conducting, the flux $H^{*}$ is a macroscopic quantity that gives an upper bound on the strength of the magnetic field, above which the nature of the superconducting state changes, in the sense that the superconducting medium is then penetrated by a stationary magnetic field (Shubnikov phase). It has been observed that this penetration of the superconducting medium always starts at the boundary. Hence, it is the objective to develop a magnetic environment for a thin superconducting film such that the Meissner state is stabilized for transport currents as high as possible. Ideally this would mean to have a homogeneous current density of strength $j_{0}-\varepsilon$. As the magnetic field of the thin superconducting film is affected by the magnetic self-field of the permanent magnets that build the magnetic environment, their shape may be used to control the distribution of the current density $j$ inside the film. In other words, given a superconducting film $\Gamma$ and a total transport current $I$, it is the objective to

Find a shape for the magnetic environment such that $j \approx$ const.

It will be the main subject of the second part of this work to analyze the Geometric Optimization Problem stated above in mathematically rigorous terms, and to provide according numerical approximation schemes with appropriate examples. In the discussion of a proposed approximation scheme using explicit boundary representation, we will identify certain drawbacks such as topological stiffness or separation problems. This mainly motivates the chances for a second approach, where then the geometric optimization problem is realized in the methodology of implicit boundary representation

The work at hand is organized as follows.

In Chapter 1 we shortly review basic concepts from classical potential theory, and state and prove a version of Green's Theorem for bounded domains of class $C^{1}$ that contain an open arc in their interior. We present layer potentials over closed 
curves as an important class of harmonic functions and concern ourselves with the single-layer potential for an open arc $\Gamma$, which is also known in the literature, but lacks a comprehensive description up to now. To this end we introduce the function space $C^{*}(\Gamma)$ in Definition 1.13 as the appropriate space for the densities of the single-layer potential. In this setting we state and prove basic properties of $C^{*}(\Gamma)$ and the single-layer potential for the open arc. The chapter is closed with the examination of a specific integral operator $L$ which will be a central ingredient for the existence analysis of Chapter 2.

In Chapter 2 we consider the Boundary Value Problem for the Laplace equation that arises from the modelling process described in the introduction. We formulate the problem rigorously in Problem 2.1 and prove uniqueness with the proof being based on the version of Green's Theorem from Chapter 1. The existence of a solution is established following the classical approach via boundary integral equations. Using the cosine substitution as the key tool, we derive an operator equation of the second kind which is well-posed due to the invertibility of $L$ (see Theorem 2.10). The chapter is concluded by identifying the jump $\left[\frac{\partial u}{\partial \nu_{\Gamma}}\right]$ of the normal derivative of the solution across $\Gamma$ as a current distribution for $\Gamma$. Following an idea of Hayashi [34] from 1973, we show in Theorem 2.15 that $\left[\frac{\partial u}{\partial \nu_{\Gamma}}\right]$ is the solution to a Cauchy type integral equation, for which a characterization in terms of the behaviour at the endpoints of $\Gamma$ can be obtained.

Chapter 3 deals with the analysis of the Geometric Optimization Problem that has been formulated above in loose terms. We state the Geometric Optimization Problem in mathematical terms in Problem 3.3, deriving the $L_{2}$-type objective functional

$$
F\left(\partial D_{\theta}\right):=\frac{1}{2} \int_{\Gamma} w(x)\left[\widetilde{F}\left(\partial D_{\theta}\right)-\frac{I}{|\Gamma|}\right]^{2} d s
$$

where $\widetilde{F}$ the current mapping that relates an admissible domain $D_{\theta}$ with shape parameter $\theta$ to the current distribution for the associated Boundary Value Problem. The choice of $F$ is subsequently justified from an applicational point of view.

In Section 3.2 and Section 3.3 we prepare the sensitivity analysis by deriving the factorization (3.13) for $F$, and by compiling classical and establishing spezialized results on Fréchet differentiability for parameter dependent operators.

Based on these abstract results we concern ourselves in Section 3.4 with the sensitivity analysis for the solution to the Boundary Value Problem. The key result is the differentiability of the double-layer potential with respect to perturbations of the domain (Theorem 3.28) similar to the work of Potthast [58]. The sensitivity result Theorem 3.33 then constitutes the first of three central results of this thesis, stating the differentiable dependence of the solution to the Boundary Value Problem on the shape parameter. 
In Theorem 3.36 a sensitivity result for the functional $F$ is derived, which forms the second central result. It is based on the results of Section 3.4 and the factorization (3.13). The chapter is concluded by Theorem 3.37 which is the third central result of this thesis. It states the existence of a minimizer for the Geometric Optimization Problem 3.3 under suitable assumptions on the shape parameter.

Chapter 4 is concerned with three approximation schemes. As a necessary basis, we present in Section 4.1 a combined collocation and quadrature method as an approximation scheme for the solution to the Boundary Value Problem. For this standard approach we also adopt the convergence analysis from [35].

The Steepest Descent Algorithm in Section 4.2 is a first numerical approach to the Geometric Optimization Problem relying on explicit boundary representation. We assume the boundary to be given by a parametrization in polar coordinates with the radial function being either a trigonometric polynomial or a linear combination of specially designed radial basis functions (4.19). Approaches of this kind are well-known from the literature.

In Section 4.3 we present a Level Set Algorithm as an alternative approach that is based on implicit boundary representation. To combine the differentiability results from Chapter 3 with the level set idea that has been described in the introduction, we derive two specially designed algorithms. The first one resolves the boundary in the context of a level set method, the second one extends the 'boundary' velocity to the level set grid. Furthermore, Section 4.3 gives an introduction to the level set idea in general and provides the reader with a description of the other algorithms from the literature that are used in the context of our algorithm.

The feasibility of the presented numerical algorithms for solving the Boundary Value Problem 2.1 and the Geometric Optimization Problem 3.3 is verified in Chapter 5, where we give the according numerical examples. Furthermore, we discuss the results from a physical point of view presenting an example for the behaviour of the magnetic field and the current distribution for a simple geometry. The observations are then compared with the corresponding behaviour of the solutions to the Geometric Optimization Problem that we obtain from the different methods described in Chapter 4.

The work is concluded with a comparison of the advantages and drawbacks of explicit and implicit boundary representation and an outlook in Chapter 6 . 


\section{Chapter 1}

\section{Potential theory}

In this chapter we present a compilation of some of the central results from potential theory that contribute directly to this work. We follow [47] and [31] in our description. We will moreover adapt and extend the results that have been present in the literature for the case of domains to the situation of cracks and domains with cracks.

\subsection{Harmonic functions}

We start our presentation by introducing the notion of harmonic functions.

Definition 1.1. A twice continuously differentiable, real-valued function $u$ on a domain $\Omega \subset \mathbb{R}^{2}$ is called harmonic, if it satisfies Laplace's equation

$$
\Delta u=0 \text { in } \Omega
$$

where

$$
\Delta u:=\frac{\partial^{2} u}{\partial x_{1}^{2}}+\frac{\partial^{2} u}{\partial x_{2}^{2}}
$$

Many properties of harmonic functions rely on Green's Theorem. We state it here together with two corollaries as it will be the key ingredient for the uniqueness theorem of Section 2.2.

Theorem 1.2 (Green's Theorem). Let $\Omega \subset \mathbb{R}^{2}$ be a bounded domain of class $C^{1}$ and let $\nu$ be the unit normal to the boundary $\partial \Omega$ directed into the exterior of $\Omega$. Furthermore, let $u \in C^{2}(\bar{\Omega})$ and $v \in C^{1}(\bar{\Omega})$. Then $u$ and $v$ satisfy Green's first theorem

$$
\int_{\Omega}\{\langle\operatorname{grad} v, \operatorname{grad} u\rangle+v \Delta u\} d x=\int_{\partial \Omega} v \frac{\partial u}{\partial \nu} d s .
$$


Proof. see [47], p. 69.

We observe that Green's theorem also holds under weaker conditions on the boundary regularity of $\Omega$. In particular, $\Omega$ is allowed to have finitely many corners. For a more detailed study we refer to [49] or [50].

From Green's Theorem we can immediately infer an additional property of harmonic functions in the interior of $\Omega$, which will be stated in the following corollary.

Corollary 1.3. Let $\Omega \subset \mathbb{R}^{2}$ be a bounded domain of class $C^{1}$, let $\nu$ denote the unit normal to the boundary $\partial \Omega$ directed into the exterior of $\Omega$, and let $u \in C^{2}(\bar{\Omega})$ be harmonic. Then

$$
\int_{\partial \Omega} \frac{\partial u}{\partial \nu} d s=0
$$

Proof. Apply Theorem 1.2 to $u$ and $v \equiv 1$.

Corollary 1.3 can be formulated analogously for the exterior with the additional requirement that $u$ is bounded in the unbounded exterior.

Corollary 1.4. Let $\Omega \subset \mathbb{R}^{2}$ be a bounded, simply connected domain of class $C^{1}$ and let $\nu$ be the unit normal to the boundary $\partial \Omega$ directed into the exterior of $\Omega$. Furthermore, let $u \in C^{2}\left(\mathbb{R}^{2} \backslash \Omega\right)$ be harmonic and bounded in $\mathbb{R}^{2} \backslash \Omega$. Then

$$
\int_{\partial \Omega} \frac{\partial u}{\partial \nu} d s=0
$$

Proof. see [47], pp. 69.

In the modelling process from the introduction we have come across harmonic functions that are defined in the exterior of an open arc. To analyze the behaviour of these functions adequately, we will formulate and prove a version of Green's Theorem for domains that contain an open arc in the interior. To this end, let us first define rigorously what we mean by an open arc.

Definition 1.5. Let $\gamma:[a, b] \rightarrow \mathbb{R}^{2}$ be injective, regular (i.e. $\gamma^{\prime} \neq 0$ ), and $k$-times differentiable. Then

$$
\Gamma:=\gamma([a, b]) \subset \mathbb{R}^{2}
$$

is called an open arc of class $C^{k}$. The endpoints of $\Gamma$ will be denoted by $z_{a}:=\gamma(a)$ and $z_{b}:=\gamma(b)$, the unit normal $\nu_{\Gamma}$ to $\Gamma$ will be given by

$$
\nu_{\Gamma}(x):=\left(\begin{array}{cc}
0 & -1 \\
1 & 0
\end{array}\right) \frac{\gamma^{\prime}(t)}{\left\|\gamma^{\prime}(t)\right\|}, \quad x=\gamma(t), t \in[a, b] .
$$


For the case of $\Gamma$ being parametrized by arclength, i.e. $\left\|\gamma^{\prime}\right\|=1$, we will also use the abbreviation

$$
\left[\gamma^{\prime}\right]^{\perp}=\left(\begin{array}{cc}
0 & -1 \\
1 & 0
\end{array}\right) \gamma^{\prime}(t), \quad t \in[a, b]
$$

for the normal $\nu_{\Gamma}$.

Theorem 1.6. Let $\Omega \subset \mathbb{R}^{2}$ be a bounded domain of class $C^{1}$ containing an open arc $\Gamma$ of class $C^{3}$ in its interior, and let $\nu_{\Omega}$ be the unit normal directed into the exterior of $\Omega$. Let $u \in C^{2}(\bar{\Omega} \backslash \Gamma) \cap C(\bar{\Omega})$ be harmonic in $\bar{\Omega} \backslash \Gamma$ and satisfy $u=0$ on $\Gamma$. Then

$$
\operatorname{grad} u \in L^{2}(\Omega)
$$

and the following version of Green's Theorem holds:

$$
\int_{\Omega}|\operatorname{grad} u|^{2} d x=\int_{\partial \Omega} u \frac{\partial u}{\partial \nu} d s .
$$

Proof. For the first statement we follow [18, 46, 51]. We choose a monotonically increasing, odd function $\psi \in C^{1}(\mathbb{R})$ that satisfies $\psi(t)=0$ in $[-1,1]$ and $\psi(t)=t$ for $|t| \geq 2$. Then we define functions

$$
u_{n}:=\frac{\psi(n u)}{n}
$$

noting that all $u_{n}$ belong to $C^{1}(\Omega)$, vanish in a neighbourhood $U_{n}$ of $\Gamma$ and converge uniformly to $u$ on $\bar{\Omega}$.

Picking a smooth, simply connected subset $S_{n}$ of $U_{n}$ that contains $\Gamma$, we can apply Green's theorem to $u$ and $u_{n}$ in $\bar{\Omega} \backslash S_{n}$ to find

$$
\begin{aligned}
\int_{\Omega \backslash S_{n}}\left\langle\operatorname{grad} u_{n}, \operatorname{grad} u\right\rangle d x-\int_{\Omega \backslash S_{n}} u_{n} \Delta u d x \\
=\int_{\partial \Omega} u_{n} \frac{\partial u}{\partial \nu_{\Omega}} d s-\int_{\partial S_{n}} u_{n} \frac{\partial u}{\partial \nu_{S_{n}}} d s
\end{aligned}
$$

where $\nu_{S_{n}}$ denotes the exterior unit normal to $S_{n}$. Now the second term on the right hand side of (1.6) vanishes since $u_{n} \equiv 0$ on $\partial S_{n} \subset U_{n}$. Similarly the second term on the left hand side vanishes due to $\Delta u=0$ on $\left(\bar{\Omega} \backslash S_{n}\right) \subset(\bar{\Omega} \backslash \Gamma)$.

Moreover, grad $u_{n}$ vanishes identically on $S_{n}$, where it is defined on the complement of $\Gamma$ which is of measure zero. Consequently, for each $n \in \mathbb{N}$ equation (1.6) reduces to

$$
\int_{\Omega}\left\langle\operatorname{grad} u_{n}, \operatorname{grad} u\right\rangle d x=\int_{\partial \Omega} u_{n} \frac{\partial u}{\partial \nu_{\Omega}} d s
$$


where the integrals are understood in the Lebesgue sense. Turning to the integrand on the left hand side of (1.7) we calculate

$$
\operatorname{grad} u_{n}=\psi^{\prime}(n u) \operatorname{grad} u \text {. }
$$

This yields pointwise convergence for

$$
\left\langle\operatorname{grad} u_{n}, \operatorname{grad} u\right\rangle \rightarrow|\operatorname{grad} u|^{2}, \quad x \in \bar{\Omega} \backslash M,
$$

where $M:=\Gamma \cup\{x \in \bar{\Omega} \backslash \Gamma: u(x)=0$, grad $u(x) \neq 0\}$ is of measure zero. From the properties of $\psi$ and (1.8) we see that $\left\langle\operatorname{grad} u_{n}, \operatorname{grad} u\right\rangle$ is non-negative on the set $\bar{\Omega} \backslash \Gamma$, so that with the help of Fatou's lemma we find

$$
\begin{aligned}
\int_{\Omega}|\operatorname{grad} u|^{2} d x & =\int_{\Omega} \lim _{n \rightarrow \infty}\left\langle\operatorname{grad} u_{n}, \operatorname{grad} u\right\rangle d x \\
& \leq \lim _{n \rightarrow \infty} \int_{\Omega}\left\langle\operatorname{grad} u_{n}, \operatorname{grad} u\right\rangle d x \\
& =\lim _{n \rightarrow \infty} \int_{\partial \Omega} u_{n} \frac{\partial u}{\partial \nu_{\Omega}} d s .
\end{aligned}
$$

Due to the positivity of the left hand side we can estimate the right hand side further using the triangle inequality and the fact that the $u_{n}$ converge uniformly. We obtain

$$
\lim _{n \rightarrow \infty} \int_{\partial \Omega} u_{n} \frac{\partial u}{\partial \nu_{\Omega}} d s \leq \lim _{n \rightarrow \infty}\left\|u_{n}-u\right\|_{\infty, \partial \Omega} \int_{\partial \Omega}\left|\frac{\partial u}{\partial \nu_{\Omega}}\right| d s+\left|\int_{\partial \Omega} u \frac{\partial u}{\partial \nu_{\Omega}} d s\right|<\infty .
$$

This then completes the proof of the first statement.

For the second statement we observe that (1.8) yields the estimate

$$
\left|\left\langle\operatorname{grad} u_{n}, \operatorname{grad} u\right\rangle\right| \leq\left\|\psi^{\prime}\right\|_{\infty}|\operatorname{grad} u|^{2}
$$

on $\bar{\Omega} \backslash M$, where the right hand side is integrable due to the first statement. Now the pointwise convergence (1.9) allows us to employ Lebesgue's dominated convergence theorem to obtain

$$
\begin{aligned}
\int_{\Omega}|\operatorname{grad} u|^{2} d x & =\lim _{n \rightarrow \infty} \int_{\Omega}\left\langle\operatorname{grad} u_{n}, \operatorname{grad} u\right\rangle d x \\
& =\lim _{n \rightarrow \infty} \int_{\partial \Omega} u_{n} \frac{\partial u}{\partial \nu_{\Omega}} d s .
\end{aligned}
$$

Then (1.5) follows by interchanging the integration and taking the limit in the right hand side of the above, which can be done due to the uniform convergence of $u_{n}$ to $u$ on $\bar{\Omega}$. 
The statement of Green's Theorem both in the classical case of Theorem 1.2 as well as in the case of domains with cracks (Theorem 1.6) requires differentiability of the solution up to the boundary. For the Boundary Value Problem we are going to consider in Chapter 2 we would like to relax these requirements. In order to achieve this, we need the concept of parallel curves from differential geometry. We state the central properties of parallel curves in the following lemma.

Lemma 1.7. Let $\Gamma$ be a regular, injective, open arc of class $C^{k}, k \geq 2$, with unit normal $\nu_{\Gamma}$. Then for $h>0$ sufficiently small the parallel arcs

$$
\Gamma_{ \pm, h}:=\left\{x \pm h \nu_{\Gamma}(x) \in \mathbb{R}^{2}: x \in \Gamma\right\}
$$

are also regular, injective and open. They are of class $C^{k-1}$ with the unit normal $\nu_{\Gamma, h}(y)=\nu_{\Gamma}(x)$ for $y=x \pm h \nu_{\Gamma}(x)$. Furthermore the line element on $\Gamma_{ \pm, h}$ can be expressed in terms of $\Gamma$ by

$$
d s(y)=|1 \mp h \kappa(x)| d s(x),
$$

where $\kappa$ denotes the oriented curvature on $\Gamma$.

Proof. Consider arclength parametrization $\gamma:\left[0, L_{\Gamma}\right] \rightarrow \mathbb{R}^{2}$ for $\Gamma$, and observe that $\Gamma_{+, h}$ can be also parametrized depending linearly on $\gamma$ through

$$
\widetilde{\gamma}=\gamma+h\left[\gamma^{\prime}\right]^{\perp}
$$

Furthermore, we have by differentiating the equation $\left\|\gamma^{\prime}\right\|=1$ that $\left\langle\gamma^{\prime \prime}, \gamma^{\prime}\right\rangle=0$ which implies

$$
\gamma^{\prime \prime}=\left\langle\gamma^{\prime \prime},\left[\gamma^{\prime}\right]^{\perp}\right\rangle\left[\gamma^{\prime}\right]^{\perp}
$$

By differentiating (1.12) and inserting (1.13) it follows that

$$
\begin{aligned}
\widetilde{\gamma}^{\prime} & =\gamma^{\prime}+h\left[\gamma^{\prime \prime}\right]^{\perp} \\
& =\gamma^{\prime}+h\left\langle\gamma^{\prime \prime},\left[\gamma^{\prime}\right]^{\perp}\right\rangle\left[\left[\gamma^{\prime}\right]^{\perp}\right]^{\perp} \\
& =\gamma^{\prime}-h\left\langle\gamma^{\prime \prime},\left[\gamma^{\prime}\right]^{\perp}\right\rangle \gamma^{\prime} \\
& =(1-h \kappa) \gamma^{\prime}
\end{aligned}
$$

as $\kappa=\operatorname{sign}\left(\left\langle\gamma^{\prime \prime},\left[\gamma^{\prime}\right]^{\perp}\right\rangle\right)\left\|\gamma^{\prime \prime}\right\|=\operatorname{sign}\left(\left\langle\gamma^{\prime \prime},\left[\gamma^{\prime}\right]^{\perp}\right\rangle\right) \sqrt{\left\langle\gamma^{\prime \prime},\left[\gamma^{\prime}\right]^{\perp}\right\rangle^{2}}$. So, (1.12) implies that $\Gamma_{+, h}$ is of class $C^{k-1}$, and (1.14) tells that for any $\tau \in\left[0, L_{\Gamma}\right]$ the tangential directions of $\Gamma$ and $\Gamma_{+, h}$ coincide. Hence, the unit normal to $\Gamma_{h}$ in

$$
y=\widetilde{\gamma}(\tau)=\gamma(\tau)+h\left[\gamma^{\prime}(\tau)\right]^{\perp}=x+h \nu_{\Gamma}(x)
$$

is given by the unit normal to $\Gamma$ in $x=\gamma(\tau)$. Similarly, the line elements relate via (1.14) as

$$
d s(y)=\left\|\widetilde{\gamma}^{\prime}(\tau)\right\| d \tau=|1-h \kappa(\gamma(\tau))|\left\|\gamma^{\prime}(\tau)\right\| d \tau=|1-h \kappa(x)| d s(x) .
$$


Since $\kappa$ remains bounded, we observe from (1.14) that $\widetilde{\gamma}$ is regular for sufficiently small $h>0$. Injectivity and openness are also satisfied for $h$ sufficiently small. The case of $\Gamma_{-, h}$ is proved analogously.

Note that the result of Lemma 1.7 also applies to the case of closed curves $\Sigma$, in which case we think of $\Sigma$ as being parametrized from $\left[0, L_{\Sigma}\right)$ with a counterclockwise orientation. The parallel curves $\Sigma_{+, h}$ and $\Sigma_{-, h}$ are then also closed due to the periodicity of the parametrization.

\section{$1.2 \quad$ Layer potentials}

The existence analysis for the Boundary Value Problem of Section 2.1 will be based on using layer potentials as ansatz functions. The properties of these layer potentials are well-known (see, for example, [47] for a detailed description). We will therefore present just the needed properties without giving detailed proofs. Moreover, we will employ single layer potentials over an open arc. To our knowledge, there is no comprehensive description of the properties for this particular case available. We will therefore give sketches for some of the proofs.

Theorem 1.8. For $x, y \in \mathbb{R}^{2}, x \neq y$, the function

$$
\Phi(x, y):=\frac{1}{2 \pi} \ln \frac{1}{|x-y|}
$$

is called the fundamental solution to the Laplace equation in $\mathbb{R}^{2}$. For fixed $y \in \mathbb{R}^{2}$ it is harmonic in $\mathbb{R}^{2} \backslash\{y\}$.

Proof. The result follows from a direct computation.

In the context of electrostatics the fundamental solution is interpreted as an electric or magnetic monopole located at a fixed $y \in \mathbb{R}^{2}$, giving rise to an electric or magnetic potential in $\mathbb{R}^{2} \backslash\{y\}$.

Definition 1.9. Let $D \subset \mathbb{R}^{2}$ be a bounded, simply connected domain of class $C^{2}$. For a function $\varphi \in C(\partial D)$ the operator

$$
\left(\mathcal{S}^{D} \varphi\right)(x):=\int_{\partial D} \Phi(x, y) \varphi(y) d s(y), \quad x \in \mathbb{R}^{2} \backslash \partial D
$$

is called (logarithmic) single-layer potential operator with density $\varphi$. 
The single-layer potential for the electric case may be viewed as a distributed monopole, where the charge is distributed over the contour $\partial D$ according to the density $\varphi$.

Theorem 1.10. Let $D \subset \mathbb{R}^{2}$ be a bounded, simply connected domain of class $C^{2}$. Then the single-layer potential operator with density $\varphi \in C(\partial D)$ given by (1.16) has the following properties:

1. $\mathcal{S}^{D} \varphi$ is continuous in all of $\mathbb{R}^{2}$, and on the boundary $\partial D$ we have

$$
\left(\mathcal{S}^{D} \varphi\right)(x)=\int_{\partial D} \Phi(x, y) \varphi(y) d s(y), \quad x \in \partial D
$$

where the integral exists as an improper integral.

2. $\mathcal{S}^{D} \varphi$ is harmonic in $\mathbb{R}^{2} \backslash \partial D$.

3. For the normal derivative on the boundary $\partial D$ we have

$$
\frac{\partial\left(\mathcal{S}^{D} \varphi\right)_{ \pm}}{\partial \nu}(x)=\int_{\partial D} \frac{\partial \Phi(x, y)}{\partial \nu(x)} \varphi(y) d s(y) \mp \frac{1}{2} \varphi(x), \quad x \in \partial D,
$$

where

$$
\frac{\partial\left(\mathcal{S}^{D} \varphi\right)_{ \pm}}{\partial \nu}(x):=\lim _{h \searrow 0}\left\langle\nu(x), \operatorname{grad}\left(\mathcal{S}^{D} \varphi\right)(x \pm h \nu(x))\right\rangle
$$

is understood in the sense of uniform convergence on $\partial D$, and where the integral exists as an improper integral.

4. $\mathcal{S}^{D} \varphi$ is bounded if and only if the density satisfies

$$
\int_{\partial D} \varphi d s=0
$$

Proof. See [47], pp. 78, for the first three properties. For the necessity of the fourth property we apply Corollaries 1.3 and 1.4 to interior and exterior parallel surfaces of $\partial D$ and then use the jump relation of the third property to derive the condition on the density. The sufficiency of (1.19) can be seen from the asymptotic behaviour

$$
\Phi(x, y)=\frac{1}{2 \pi} \ln \frac{1}{|x|}+\mathcal{O}\left(\frac{1}{|x|}\right)
$$

of the fundamental solution, which holds uniformly for all $y \in \partial D$. 
Definition 1.11. Let $D \subset \mathbb{R}^{2}$ be a bounded, simply connected domain of class $C^{2}$ with unit normal $\nu_{D}$ to the boundary $\partial D$ pointing into the exterior of $D$. For a function $\varphi \in C(\partial D)$ the operator

$$
\left(\mathcal{T}^{D} \varphi\right)(x):=\int_{\partial D} \frac{\partial \Phi(x, y)}{\partial \nu_{D}(y)} \varphi(y) d s(y), \quad x \in \mathbb{R}^{2} \backslash \partial D,
$$

is called (logarithmic) double-layer potential operator with density $\varphi$.

Analogously to the single-layer potential the double-layer potential can be interpreted as distributed electric or magnetic dipoles, where the direction of the dipole is given by the unit normal $\nu_{D}$.

Theorem 1.12. Let $D \subset \mathbb{R}^{2}$ be a bounded, simply connected domain of class $C^{2}$. Then the double-layer potential operator with density $\varphi \in C(\partial D)$ given by (1.21) has the following properties:

1. $\mathcal{T}^{D} \varphi$ is harmonic in $\mathbb{R}^{2} \backslash \partial D$.

2. $\mathcal{T}^{D} \varphi$ can be continuously extended from $D$ to $\bar{D}$ and from $\mathbb{R}^{2} \backslash \bar{D}$ to $\mathbb{R}^{2} \backslash D$ with limiting values

$$
\left(\mathcal{T}^{D} \varphi\right)_{ \pm}(x)=\int_{\partial D} \frac{\partial \Phi(x, y)}{\partial \nu_{D}(y)} \varphi(y) d s(y) \pm \frac{1}{2} \varphi(x), \quad x \in \partial D
$$

where

$$
\left(\mathcal{T}^{D} \varphi\right)_{ \pm}(x):=\lim _{h \searrow 0}\left(\mathcal{T}^{D} \varphi\right)\left(x \pm h \nu_{D}(x)\right)
$$

and where the integral exists as an improper integral.

3. For $x \in \partial D$ let $x_{ \pm h}:=x \pm h \nu_{D}(x)$. Then $\mathcal{T}^{D} \varphi$ satisfies

$$
\lim _{h \searrow 0}\left\langle\nu_{D}(x), \operatorname{grad}\left(\mathcal{T}^{D} \varphi\right)\left(x_{+h}\right)-\operatorname{grad}\left(\mathcal{T}^{D} \varphi\right)\left(x_{-h}\right)\right\rangle=0
$$

uniformly for all $x \in \partial D$.

4. $\mathcal{T}^{D} \varphi$ is bounded on $\mathbb{R}^{2}$.

Proof. See [47], pp. 78, for the first three properties. The fourth property follows again from the asymptotic behaviour

$$
\operatorname{grad} \Phi(x, y)=\mathcal{O}\left(\frac{1}{|x|}\right)
$$

of the fundamental solution, which holds again uniformly for $y \in \partial D$. 
In order to study the boundary value problem that arises from the physical problem described in the introduction we also need to consider potentials that are distributed over an open arc. We introduce the single-layer potential in the following. It will turn out necessary and convenient to consider densities of a specific shape when considering layer potentials over open arcs (see, for example, [46]). Therefore we introduce the following function space.

Definition 1.13. Let $\Gamma \subset \mathbb{R}^{2}$ be an open arc of class $C^{3}$ with endpoints $z_{-1}, z_{+1}$ as in Definition 1.5. Choosing a regular parametrization $\gamma:[-1,1] \rightarrow \mathbb{R}^{2}$ for $\Gamma$, we define $C^{*}(\Gamma)$ as the set of all functions $\varphi \in C\left(\Gamma \backslash\left\{z_{-1}, z_{+1}\right\}\right)$ satisfying

$$
\varphi(x)=\frac{\widetilde{\varphi}(\arccos t)}{\left|\gamma^{\prime}(t)\right| \sqrt{1-t^{2}}}, \quad x=\gamma(t), t \in(-1,1), \widetilde{\varphi} \in C_{2 \pi, e}^{0, \alpha},
$$

where $C_{2 \pi, e}^{0, \alpha}$ is the space of $2 \pi$-periodic and even functions on $\mathbb{R}$ that are bounded and uniformly Hölder continuous with Hölder exponent $\alpha \in(0,1]$.

The space $C^{*}(\Gamma)$ is introduced using a particular parametrization $\gamma$. The following lemma will show that $C^{*}(\Gamma)$ is nevertheless well-defined.

Lemma 1.14. The space $C^{*}(\Gamma)$ is independent of the choice of the parametrization $\gamma$.

Proof. Let us first note that without loss of generality we can assume that $\Gamma$ is parametrized from $[-1,1]$, as any smooth, regular parametrization $z:[a, b] \rightarrow \mathbb{R}^{2}$ of $\Gamma$ can be equivalently transformed to a regular parametrization from $[-1,1]$ of the same smoothness due to the linear diffeomorphism $G:[-1,1] \rightarrow[a, b]$ given by $G(t):=a+\frac{b-a}{2}(t+1)$.

Consequently, let $z:[-1,1] \rightarrow \mathbb{R}^{2}$ be a regular parametrization of $\Gamma$ and assume that $\varphi \in C\left(\Gamma \backslash\left\{z_{-1}, z_{+1}\right\}\right)$ satisfies

$$
\varphi(x)=\frac{\widetilde{\varphi}(\arccos t)}{\left|z^{\prime}(t)\right| \sqrt{1-t^{2}}}, \quad x=z(t), t \in(-1,1), \widetilde{\varphi} \in C_{2 \pi, e}^{0, \alpha} .
$$

Then there exists a function $h:[-1,1] \rightarrow[-1,1]$, which is three-times continuously differentiable, surjective and strictly increasing such that $z(h(t))=\gamma(t)$ for all $t \in[-1,1]$.

Let us first consider the auxiliary function

$$
f(t):=\frac{1-\cos ^{2} t}{1-(h(\cos t))^{2}}, \quad t \in(0, \pi),
$$


and prove that is has an extension in $C^{1}(\mathbb{R})$. We observe that $f$ is well-defined and as smooth as $h$ on $(0, \pi)$. Using Taylor expansions around $t=0$ and $t=\pi$ for the numerator and denominator of $f$, we see that $f$ can be continuously extended in $t=0$ by $\left(h^{\prime}(1)\right)^{-1}$ and in $t=\pi$ by $\left(h^{\prime}(-1)\right)^{-1}$, which are both different from zero due to $h$ being strictly increasing. Similarly, we obtain that the first derivative of $f$ can be continuously extended in $t=0$ and $t=\pi$ by zero. Hence, $f$ can be extended to a once continuously differentiable, even function on $\mathbb{R}$.

In the next step, we will formally represent $\varphi$ satisfying (1.24) in the form of Definition 1.13 with a function $\widetilde{\widetilde{\varphi}} \in C_{2 \pi, e}^{0, \alpha}$. Using $\gamma^{\prime}(t)=z^{\prime}(h(t)) h^{\prime}(t)$ we calculate for $x=z(h(t))$ that

$$
\begin{aligned}
\varphi(x) & =\frac{\widetilde{\varphi}(\arccos h(t))}{\left|z^{\prime}(h(t))\right| \sqrt{1-(h(t))^{2}}} \\
& =\frac{\widetilde{\varphi}(\arccos h(t))}{\left|\gamma^{\prime}(t)\right| \sqrt{1-t^{2}}} h^{\prime}(t) \sqrt{\frac{1-t^{2}}{1-(h(t))^{2}}} \\
& =\frac{\widetilde{\varphi}(\arccos t)}{\left|\gamma^{\prime}(t)\right| \sqrt{1-t^{2}}},
\end{aligned}
$$

where we have set

$$
\widetilde{\widetilde{\varphi}}(t):=\widetilde{\varphi}(\arccos h(\cos t)) h^{\prime}(\cos t) \sqrt{\frac{1-\cos ^{2} t}{1-(h(\cos t))^{2}}} .
$$

Now the statement follows if we can show that $\widetilde{\widetilde{\varphi}}$ is indeed an element of $C_{2 \pi, e}^{0, \alpha}$. For the factor

$$
h^{\prime}(\cos t) \sqrt{\frac{1-\cos ^{2} t}{1-(h(\cos t))^{2}}}=h^{\prime}(\cos t) \sqrt{f(t)}
$$

we notice immediately that it is $2 \pi$-periodic and even with the derivative

$$
\frac{1}{2} h^{\prime}(\cos t) \frac{f^{\prime}(t)}{\sqrt{f(t)}}-h^{\prime \prime}(\cos t) \sin t \sqrt{f(t)}
$$

being well-defined and continuous for all $t \in \mathbb{R}$. Hence, it is an element of $C_{2 \pi, e}^{0, \alpha}$ by compact embedding. For the first factor we calculate for $t \in(0, \pi)$

$$
(\arccos h(\cos t))^{\prime}=\frac{h^{\prime}(\cos t) \sin t}{\sqrt{1-(h(\cos t))^{2}}}=h^{\prime}(\cos t) \sqrt{f(t)}
$$

noting that the derivative remains bounded since the right-hand side can be continuously extended to $\mathbb{R}$, although the extension of the left hand side to $\mathbb{R}$ is discontinuous at the points $k \pi$ for $k \in \mathbb{Z}$ since we consider $\arccos h(\cos t)$ to 
be extended as an even function to $\mathbb{R}$. This implies that $\arccos h(\cos t) \in C_{2 \pi, e}^{0,1}$. Now the statement for $\widetilde{\widetilde{\varphi}}$ follows using the chain and product rule for Hölder continuous functions.

The existence analysis of Section 2.3 will show that the characterization for $C^{*}(\Gamma)$ appears generically. Nevertheless, the behaviour of the elements of $C^{*}(\Gamma)$ is not too obvious, when they are interpreted simply as functions on $\Gamma$, independently of the underlying parametrization $\gamma$. The following lemma will therefore discuss the integrability of elements of $C^{*}(\Gamma)$.

Lemma 1.15. For each function $\varphi \in C^{*}(\Gamma)$ the expression

$$
|\varphi(x)| \sqrt{\left|x-z_{-1}\right|\left|x-z_{+1}\right|}, \quad x \in \Gamma,
$$

is uniformly bounded. Furthermore, the integral

$$
\int_{\Gamma}|\varphi| d s
$$

exists, whereas the integral

$$
\int_{\Gamma}|\varphi|^{2} d s
$$

exists if and only if the related function $\widetilde{\varphi} \in C_{2 \pi, e}^{0, \alpha}$ vanishes at the endpoints $z_{-1}$ and $z_{+1}$.

Proof. To see the condition (1.27) we parametrize $\Gamma$ by $\gamma:[-1,1] \rightarrow \mathbb{R}^{2}$ according to Definition 1.13, and obtain

$$
|\varphi(x)| \sqrt{\left|x-z_{-1}\right|\left|x-z_{+1}\right|} \leq \frac{|\widetilde{\varphi}(\arccos t)|}{\left|\gamma^{\prime}(t)\right|} \sqrt{\frac{|\gamma(t)-\gamma(-1)||\gamma(t)-\gamma(1)|}{1-t^{2}}} .
$$

As $0<m \leq\left|\gamma^{\prime}(t)\right|$ for all $t \in[-1,1]$ due to $\gamma$ being regular, the first factor on the right-hand side can be uniformly bounded by $C:=m^{-1}\|\widetilde{\varphi}\|_{\infty}$. The boundedness of the second factor on the right-hand side is derived from the Taylor expansions

$$
\gamma(t)-\gamma(-1)=(1+t) \int_{0}^{1} \gamma^{\prime}(-1+\lambda(t+1)) d \lambda
$$

as well as

$$
\gamma(1)-\gamma(t)=(1-t) \int_{0}^{1} \gamma^{\prime}(t+\lambda(1-t)) d \lambda
$$

by substitution into the numerator. The second factor then reads

$$
\left(\left|\int_{0}^{1} \gamma^{\prime}(-1+\lambda(t+1)) d \lambda\right|\left|\int_{0}^{1} \gamma^{\prime}(t+\lambda(1-t)) d \lambda\right|\right)^{\frac{1}{2}}
$$


and is obviously bounded as each component of $\gamma^{\prime}$ is bounded and continuous.

The $L_{1}$ property of $\varphi$ can be established directly from Definition 1.13 by parametrizing $\Gamma$ by $\gamma:[-1,1] \rightarrow \mathbb{R}^{2}$. Substituting $t=\cos s$ it follows that

$$
\int_{\Gamma}|\varphi| d s=\int_{-1}^{1} \frac{|\widetilde{\varphi}(\arccos t)|}{\left|\gamma^{\prime}(t)\right| \sqrt{1-t^{2}}}\left|\gamma^{\prime}(t)\right| d t=\int_{0}^{\pi} \frac{|\widetilde{\varphi}(s)|}{\sqrt{1-\cos ^{2} s}} \sin s d s \leq \pi\|\widetilde{\varphi}\|_{\infty} .
$$

For the square integrability we transform the integral as before using the parametrization $\gamma$ and substituting $t=\cos s$ to obtain

$$
\int_{\Gamma}|\varphi|^{2} d s=\int_{0}^{\pi} \frac{|\widetilde{\varphi}(s)|^{2}}{\left|\gamma^{\prime}(\cos s)\right||\sin s|} d s
$$

where $\widetilde{\varphi} \in C_{2 \pi, e}^{0, \alpha}$ is the function associated to $\varphi \in C^{*}(\Gamma)$ as in Definition 1.13.

Let us first consider sufficiency, and let $\widetilde{\varphi}$ satisfy $\widetilde{\varphi}(0)=0$. Then we can estimate

$$
|\widetilde{\varphi}(t)|=|\widetilde{\varphi}(t)-\widetilde{\varphi}(0)| \leq M_{\widetilde{\varphi}}|t|^{\alpha}
$$

for some constant $M_{\widetilde{\varphi}}>0$ and $\alpha \in(0,1]$. Using the series expansion of the sine we can estimate the integrand on the right-hand side of (1.28) for $s>0$ by

$$
\frac{|\widetilde{\varphi}(s)|^{2}}{\left|\gamma^{\prime}(\cos s)\right||\sin s|} \leq \frac{M_{\widetilde{\varphi}}^{2}}{m} s^{2 \alpha-1} f_{0}(s),
$$

where $f_{0}$ is continuous and bounded in a neighbourhood of $s=0$. As $\alpha$ is positive the right-hand side of (1.29) is improperly integrable with respect to zero. With a similar argument using $\widetilde{\varphi}(\pi)=0$ we see that the integrand on the right hand side of (1.28) also in a neighbourhood of $\pi$ has an improperly integrable majorante. Combining both arguments we obtain that the integral $\int_{\Gamma}|\varphi|^{2} d s$ is finite.

For necessity we assume that $\widetilde{\varphi}(0) \neq 0$. Then there exists $\delta>0$ such that $|\widetilde{\varphi}(s)|^{2} \geq \frac{1}{2}|\widetilde{\varphi}(0)|^{2}>0$ for all $s \in[0, \delta]$. Furthermore, $\left|\gamma^{\prime}(\cos s)\right| \leq M$ for some constant $M$ since $\gamma$ is continuously differentiable. Hence, we can estimate the integrand in the right-hand side of $(1.28)$ on $[0, \delta]$ from below by

$$
\frac{|\widetilde{\varphi}(s)|^{2}}{\left|\gamma^{\prime}(\cos s)\right||\sin s|} \geq \frac{|\widetilde{\varphi}(0)|^{2}}{2 M} \sin ^{-1} s
$$

Noting that the antiderivative $\ln \tan \frac{s}{2}$ of $\sin ^{-1} s$ is unbounded near $s=0$ we have found a minorante for (1.28) in a neighbourhood of zero which is non-integrable. The statement then follows by combining the above with a similar argument for the case of $\widetilde{\varphi}(\pi) \neq 0$. 
The property (1.27) of the elements of $C^{*}(\Gamma)$ is now sufficient to guarantee a nice behaviour of the single-layer potential. Thus, we will denote by $C^{-\frac{1}{2}}(\Gamma)$ the space of all functions $\psi \in C\left(\Gamma \backslash\left\{z_{-1}, z_{+1}\right\}\right)$ for which (1.27) holds. Note in particular that for this reason the elements of $C^{-\frac{1}{2}}(\Gamma)$ are allowed to develop singularities of order $-\frac{1}{2}$ in the endpoints of the arc. This means that $\psi \in C^{-\frac{1}{2}}(\Gamma)$ may be written in the form

$$
\psi(x)=\frac{\widehat{\psi}(x)}{\sqrt{\left|x-z_{-1}\right|\left|x-z_{+1}\right|}}, \quad x \in \Gamma \backslash\left\{z_{-1}, z_{+1}\right\},
$$

for some bounded, continuous function $\widehat{\psi}$ on $\Gamma$. We now define the single-layer potential over the open arc for densities $\psi \in C^{-\frac{1}{2}}(\Gamma)$.

Definition 1.16. Let $\Gamma \subset \mathbb{R}^{2}$ be an open arc of class $C^{3}$ with endpoints $z_{-1}, z_{+1}$. For a function $\varphi \in C^{-\frac{1}{2}}(\Gamma)$ the operator

$$
\left(\mathcal{S}^{\Gamma} \varphi\right)(x):=\int_{\Gamma} \Phi(x, y) \varphi(y) d s(y), \quad x \in \mathbb{R}^{2} \backslash \Gamma
$$

is called (logarithmic) single-layer potential operator with density $\varphi$.

The physical interpretation of $\mathcal{S}^{\Gamma} \varphi$ is analogous to the case of a closed boundary contour. Formally, the difference between the case of a closed contour and the case of an open arc is that the integral (1.31) exists only as an improper integral with respect to the endpoints of $\Gamma$. This is due to the possibly singular behaviour of the density at the endpoints $z_{ \pm 1}$ stated above.

Theorem 1.17. Let $\Gamma \subset \mathbb{R}^{2}$ be an open arc of class $C^{3}$ with endpoints $z_{-1}, z_{+1}$. Then the single-layer potential operator with density $\varphi \in C^{-\frac{1}{2}}(\Gamma)$ given by (1.31) has the following properties:

1. $\mathcal{S}^{\Gamma} \varphi$ is continuous in all of $\mathbb{R}^{2}$, and on $\Gamma$ we have

$$
\left(\mathcal{S}^{\Gamma} \varphi\right)(x)=\int_{\Gamma} \Phi(x, y) \varphi(y) d s(y), \quad x \in \Gamma
$$

where the integral exists as an improper integral.

2. $\mathcal{S}^{\Gamma} \varphi$ is harmonic in $\mathbb{R}^{2} \backslash \Gamma$.

3. For the normal derivative on $\Gamma$ we have

$$
\frac{\partial\left(\mathcal{S}^{\Gamma} \varphi\right)_{ \pm}}{\partial \nu}(x)=\int_{\Gamma} \frac{\partial \Phi(x, y)}{\partial \nu(x)} \varphi(y) d s(y) \mp \frac{1}{2} \varphi(x)
$$


for all $x \in \Gamma \backslash\left\{z_{-1}, z_{+1}\right\}$, where

$$
\frac{\partial\left(\mathcal{S}^{\Gamma} \varphi\right)_{ \pm}}{\partial \nu}(x):=\lim _{h \searrow 0}\left\langle\nu(x), \operatorname{grad}\left(\mathcal{S}^{\Gamma} \varphi\right)(x \pm h \nu(x))\right\rangle
$$

is understood in the sense of locally uniform convergence on $\Gamma$, and where the integral exists as an improper integral.

4. $\mathcal{S}^{\Gamma} \varphi$ is bounded if the density satisfies

$$
\int_{\Gamma} \varphi d s=0
$$

Proof. The second property is proved in the same way as in Theorem 1.10, noting that the density $\varphi \in C^{-\frac{1}{2}}(\Gamma)$ is integrable.

Introducing the cut-off function

$$
h(t):= \begin{cases}0, & t \in\left[0, \frac{1}{2}\right] \\ 2 t-1, & t \in\left[\frac{1}{2}, 1\right] \\ 1, & t \in[1, \infty)\end{cases}
$$

and extending $\Gamma$ to a simple, closed curve $\widetilde{\Gamma}$ of class $C^{2}$ we define functions $u_{n}:=\mathcal{S}^{\widetilde{\Gamma}} \varphi_{n}$ through

$$
\varphi_{n}(x):= \begin{cases}\varphi(x) h\left(n\left|x-z_{-1}\right|\right) h\left(n\left|x-z_{+1}\right|\right), & x \in \Gamma, \\ 0, & x \in \widetilde{\Gamma} \backslash \Gamma .\end{cases}
$$

Then by the first property of Theorem 1.10 the functions $u_{n}$ are continuous on $\mathbb{R}^{2}$ and their values on $\Gamma$ are given by (1.32) with density $\varphi_{n}$. Now the first property follows since for $\varphi \in C^{-\frac{1}{2}}(\Gamma)$ we have uniform convergence of $u_{n}$ to $\left(\mathcal{S}^{\Gamma} \varphi\right)$ on compact subsets of $\mathbb{R}^{2}$ (see, for example, [36, 47] for more elaborate proofs).

The proof of the jump relation (1.33) again uses the cut-off function $h$ and the densities $\varphi_{n}$ on the closed curve $\widetilde{\Gamma}$. For any compact subset of $\Gamma^{*} \subset \Gamma \backslash\left\{z_{-1}, z_{+1}\right\}$ we can find $N \in \mathbb{N}$ sufficiently large, such that the neighbourhoods of the endpoints $z_{ \pm 1}$ given by

$$
\Gamma_{N, \pm}:=\left\{x \in \Gamma: N\left|x-z_{ \pm 1}\right| \leq 1\right\}
$$

are connected and satisfy $\Gamma^{*} \cap\left(\Gamma_{N,-} \cup \Gamma_{N,+}\right)=\emptyset$. Then for any $x \in \Gamma^{*}$ and $z:=x \pm h \nu_{\Gamma}(x)$ with $h>0$ sufficiently small we split the derivative of $S^{\Gamma} \varphi$ in direction $\nu_{\Gamma}(x)$ for the point $z \notin \Gamma$ using $\varphi_{N}$ into 


$$
\begin{aligned}
\left\langle\nu_{\Gamma}(x)\right. & \left., \operatorname{grad}\left(S^{\Gamma} \varphi\right)(z)\right\rangle \\
& =\left\langle\nu_{\Gamma}(x), \operatorname{grad}\left(S^{\Gamma} \varphi_{N}\right)(z)\right\rangle+\left\langle\nu_{\Gamma}(x), \operatorname{grad}\left(S^{\Gamma}\left(\varphi-\varphi_{N}\right)\right)(z)\right\rangle .
\end{aligned}
$$

Considering the first term on the right hand side of (1.36), we see that it coincides with the analogous expression with $S^{\Gamma} \varphi_{N}$ exchanged against $S^{\widetilde{\Gamma}} \varphi_{N}$ as $\varphi_{N}$ can be interpreted as a continuous density on the closed curve $\widetilde{\Gamma}$. Hence, we can apply Theorem 1.10 to the first term on the right-hand side of (1.36), and we obtain for $x \in \Gamma^{*}$

$$
\begin{aligned}
\frac{\partial\left(\mathcal{S}^{\Gamma} \varphi_{N}\right)_{ \pm}}{\partial \nu}(x) & =\lim _{h \searrow 0}\left\langle\nu_{\Gamma}(x), \operatorname{grad}\left(S^{\widetilde{\Gamma}} \varphi_{N}\right)\left(x \pm h \nu_{\Gamma}(x)\right)\right\rangle \\
& =\int_{\Gamma} \frac{\partial \Phi(x, y)}{\partial \nu(x)} \varphi_{N}(y) d s(y) \mp \frac{1}{2} \varphi(x)
\end{aligned}
$$

in the sense of uniform convergence on $\Gamma^{*}$, where we have used again that the integration reduces from $\widetilde{\Gamma}$ to $\Gamma$ as $\varphi_{N}$ vanishes on $\widetilde{\Gamma} \backslash \Gamma$. Moreover, we have used that $\varphi_{N}$ and $\varphi$ coincide on the complement of the neighbourhoods $\Gamma_{N, \pm}$ of the endpoints $z_{ \pm 1}$. Hence, we have replaced $\varphi_{N}$ by $\varphi$ in the second term on the right-hand side as $\Gamma^{*} \cap\left(\Gamma_{N,-} \cup \Gamma_{N,+}\right)=\emptyset$.

Next, we consider the second term on the right-hand side of (1.36). We note again from the properties of $\varphi_{N}$ that the domain of integration for the singlelayer potential $S^{\Gamma}\left(\varphi-\varphi_{N}\right)$ does not intersect with $\Gamma^{*}$. Consequently, the gradient of $S^{\Gamma}\left(\varphi-\varphi_{N}\right)$ is well-defined and continuous on $\Gamma^{*}$, and it can be obtained by interchanging differentiation and integration. Thus, we obtain for $x \in \Gamma^{*}$

$$
\begin{aligned}
\frac{\partial\left(\mathcal{S}^{\Gamma}\left(\varphi-\varphi_{N}\right)\right)_{ \pm}}{\partial \nu}(x) & =\lim _{h \searrow 0}\left\langle\nu_{\Gamma}(x), \operatorname{grad}\left(S^{\Gamma}\left(\varphi-\varphi_{N}\right)\right)\left(x \pm h \nu_{\Gamma}(x)\right)\right\rangle \\
& =\int_{\Gamma} \frac{\partial \Phi(x, y)}{\partial \nu(x)}\left(\varphi(y)-\varphi_{N}(y)\right) d s(y)
\end{aligned}
$$

in the sense of uniform convergence on $\Gamma^{*}$. Combining the results for the two terms on the right-hand side of (1.36) we see that also the limit $h \searrow 0$ for the left-hand side of (1.36) exists in the sense of uniform convergence on $\Gamma^{*}$, and that it is given by the sum of the corresponding terms on the right-hand side. This then implies (1.33) and proves the third property.

For the boundedness of $\left(\mathcal{S}^{\Gamma} \varphi\right)$ we appeal again to the asymptotic behaviour (1.20) of the fundamental solution as in the proof to Theorem 1.10.

The fourth property of Theorem 1.17 gives a sufficient condition for the boundedness of the single-layer potential over the open arc $\Gamma$. For any density $\varphi \in C^{-\frac{1}{2}}(\Gamma)$ 
we can modify this density by introducing the operator $M$ defined by

$$
(M \varphi)(x):=\varphi(x)-\frac{1}{|\Gamma|} \int_{\Gamma} \varphi d s, \quad x \in \Gamma \backslash\left\{z_{-1}, z_{+1}\right\},
$$

such that the modified density $M \varphi$ satisfies (1.34). Accordingly, we will call single-layer potentials with densities of the shape of (1.37) modified single-layer potentials.

\subsection{An invertible integral operator}

Layer potentials over closed contours may also be considered in spaces with higher regularity. In this section we will set off from the modified single-layer potential, for which it can be proven that it is invertible in the space of Hölder continuous functions. Based on this invertible operator we will deduce the invertibility of an integral operator defined on $[0, \pi]$ for functions in $C_{2 \pi, e}^{0, \alpha}$.

Theorem 1.18. Let $\mathcal{C} \subset \mathbb{R}^{2}$ be a closed, simple curve of class $C^{2}$. Then the modified single-layer potential $S_{0}: C^{0, \alpha}(\mathcal{C}) \rightarrow C^{1, \alpha}(\mathcal{C})$ given by

$$
\left(S_{0} \varphi\right)(z):=\frac{1}{2 \pi} \int_{\mathcal{C}} \ln \frac{1}{|\zeta-z|}\left(\varphi(\zeta)-\frac{1}{|\mathcal{C}|} \int_{\mathcal{C}} \varphi d s\right) d s(\zeta)+\frac{1}{|\mathcal{C}|} \int_{\mathcal{C}} \varphi d s
$$

with a real-valued, Hölder continuous density $\varphi \in C^{0, \alpha}(\mathcal{C})$ is bijective with a bounded inverse $S_{0}^{-1}: C^{1, \alpha}(\mathcal{C}) \rightarrow C^{0, \alpha}(\mathcal{C})$.

Proof. see [47], pp. 118.

In the following lemma we will apply the result of Theorem 1.18 to the unit circle in $\mathbb{R}^{2}$ and relate the operator $S_{0}$ to an operator with a weakly singular kernel that maps functions in $C^{0, \alpha}([0,2 \pi])$ to functions in $C^{1, \alpha}([0,2 \pi])$.

Lemma 1.19. Let $\mathcal{C}=\{(\cos t, \sin t): t \in[0,2 \pi]\}$ be the unit circle in $\mathbb{R}^{2}$. Then the modified single-layer potential (1.38) coincides with the integral

$$
\frac{1}{4 \pi} \int_{0}^{2 \pi}\left[-\ln \left(4 \sin ^{2} \frac{\tau-t}{2}\right)+2\right] \widetilde{\varphi}(\tau) d \tau, \quad t \in[0,2 \pi]
$$

where $\widetilde{\varphi}(\tau):=\varphi(\cos \tau, \sin \tau)$. 
Proof. We re-arrange (1.38) such that $\varphi$ occurs only in the outer integration and parametrize $\mathcal{C}$ by $x(t):=(\cos t, \sin t)$ to obtain

$$
\left(S_{0} \widetilde{\varphi}\right)(t)=\frac{1}{4 \pi} \int_{0}^{2 \pi} k_{0}(t, \tau) \widetilde{\varphi}(\tau) d \tau
$$

where the kernel $k_{0}$ is given by

$$
k_{0}(t, \tau)=2\left|x^{\prime}(\tau)\right|\left[\ln \frac{1}{|x(\tau)-x(t)|}-\frac{1}{2 \pi} \int_{0}^{2 \pi} \ln \left(\frac{1}{|x(\sigma)-x(t)|}\right)\left|x^{\prime}(\sigma)\right| d \sigma+1\right] .
$$

Using addition theorems for the sine and cosine we find that

$$
\begin{aligned}
|x(\tau)-x(t)|^{2} & =(\cos \tau-\cos t)^{2}+(\sin \tau-\sin t)^{2} \\
& =4 \sin ^{2} \frac{\tau-t}{2} \cos ^{2} \frac{\tau+t}{2}+4 \sin ^{2} \frac{\tau-t}{2} \sin ^{2} \frac{\tau+t}{2} \\
& =4 \sin ^{2} \frac{\tau-t}{2}
\end{aligned}
$$

As $\left|x^{\prime}(\tau)\right|^{2}=(-\sin \tau)^{2}+(\cos \tau)^{2}=1$ we can simplify the expression for $k_{0}$ to

$$
k_{0}(t, \tau)=2\left[-\frac{1}{2} \ln \left(4 \sin ^{2} \frac{\tau-t}{2}\right)+\frac{1}{4 \pi} \int_{0}^{2 \pi} \ln \left(4 \sin ^{2} \frac{\sigma-t}{2}\right) d \sigma+1\right] .
$$

We observe by substituting $s=\sigma-t$ into the integral expression and using the periodicity of the sine that the integral term vanishes (see [47], Theorem 8.21). Hence, the kernel $k_{0}$ coincides with the kernel of (1.39).

With the simple representation (1.40) for $S_{0}$ in the special case of the unit circle at hand, we are now ready to introduce the integral operator mapping functions in $C_{2 \pi, e}^{0, \alpha}$ to functions in $C_{2 \pi, e}^{1, \alpha}$ and prove that it is invertible.

Corollary 1.20. The integral operator $L: C_{2 \pi, e}^{0, \alpha} \rightarrow C_{2 \pi, e}^{1, \alpha}$ given by

$$
(L \psi)(t):=\frac{1}{4 \pi} \int_{0}^{2 \pi}\left[-\ln \left(4 \sin ^{2} \frac{\tau-t}{2}\right)+2\right] \psi(\tau) d \tau
$$

is bijective with a bounded inverse $L^{-1}: C_{2 \pi, e}^{1, \alpha} \rightarrow C_{2 \pi, e}^{0, \alpha}$. On $[0, \pi]$ it has the representation

$$
(L \psi)(t)=\frac{1}{4 \pi} \int_{0}^{\pi}\left\{-\ln \left[4(\cos t-\cos \tau)^{2}\right]+4\right\} \psi(\tau) d \tau .
$$


Proof. By virtue of Theorem 1.18 and Lemma 1.19 the operator $L$ is clearly invertible as an operator from $C_{2 \pi}^{0, \alpha}$ to $C_{2 \pi}^{1, \alpha}$ by periodic extension. To establish surjectivity from $C_{2 \pi, e}^{0, \alpha}$ to $C_{2 \pi, e}^{1, \alpha}$ we consider $f \in C_{2 \pi, e}^{1, \alpha} \subseteq C_{2 \pi}^{1, \alpha}$. For such $f$ there exists $\psi \in C_{2 \pi}^{0, \alpha}$, satisfying $L \psi=f$. Using the periodicity we find

$$
f(-t)=\frac{1}{4 \pi} \int_{0}^{2 \pi}\left[-\ln \left(4 \sin ^{2} \frac{\tau-t}{2}\right)+2\right] \psi(-\tau) d \tau
$$

for all $t \in \mathbb{R}$. Now we conclude from the fact that $f$ is even, that

$$
0=f(t)-f(-t)=\frac{1}{4 \pi} \int_{0}^{2 \pi}\left[-\ln \left(4 \sin ^{2} \frac{\tau-t}{2}\right)+2\right](\psi(\tau)-\psi(-\tau)) d \tau
$$

again for all $t \in \mathbb{R}$. Since $L$ is injective it follows that

$$
\psi(\tau)-\psi(-\tau)=0
$$

for all $\tau \in \mathbb{R}$, and thus we have $\psi \in C_{2 \pi, e}^{0, \alpha}$. A comparison of (1.42) and (1.40) for functions $\psi \in C_{2 \pi, e}^{0, \alpha}$ shows that $L$ maps even functions to even functions, whence we can conclude that $L$ is bijective as an operator from $C_{2 \pi, e}^{0, \alpha}$ to $C_{2 \pi, e}^{1, \alpha}$. The boundedness of the inverse then finally follows from Banach's Theorem.

For the representation (1.41) we split the integral (1.40) at $\pi$, use periodicity, evenness of $\psi$ and properties of the integral to obtain

$$
\begin{aligned}
(L \psi)(t)= & \frac{1}{4 \pi} \int_{0}^{\pi}\left[-\ln \left(4 \sin ^{2} \frac{\tau-t}{2}\right)+2\right] \psi(\tau) d \tau \\
& +\frac{1}{4 \pi} \int_{0}^{\pi}\left[-\ln \left(4 \sin ^{2} \frac{\tau+t}{2}\right)+2\right] \psi(\tau) d \tau .
\end{aligned}
$$

Now adding the two parts and employing the identity

$$
\ln \left[4(\cos t-\cos \tau)^{2}\right]=\ln \left(4 \sin ^{2} \frac{t-\tau}{2}\right)+\ln \left(4 \sin ^{2} \frac{t+\tau}{2}\right)
$$

for $t, \tau \in \mathbb{R}$ with $t \neq \tau$ yields (1.41). 


\section{Chapter 2}

\section{A Boundary Value Problem}

In this chapter we will analyze the Boundary Value Problem to Laplace's equation that arises from the mathematical modelling of a thin superconducting film in a magnetic environment as presented in the introduction.

After stating the problem mathematically rigorously, we will establish unique solvability for the problem. The formulation of the problem in Section 2.1 and the results of Section 2.3 are adaptations from [35], where the problem has been examined for a special case. The line of arguments in Section 2.2 differs from [35], where the uniqueness proof was based on the maximum principle for harmonic functions. In Section 2.2 we argue via Green's Theorem for domains with cracks (see Theorem 1.6) instead. The chapter will be closed by introducing the so-called current distribution. We will furthermore identify the current distribution as an inherent property of the solution and give a characterization for this property.

\subsection{Statement of the Problem}

Problem 2.1. Let $D=\bigcup_{i=1}^{n} D_{i} \subset \mathbb{R}^{2}$ be the union of finitely many open, bounded, simply connected $C^{2}$-domains, whose closures are pairwise disjoint. Define $D_{e}:=\mathbb{R}^{2} \backslash \bar{D}$ and let $\nu_{D}$ be the unit normal to $\partial D$ directed into $D_{e}$. Furthermore, let $\Gamma \subset D_{e}$ be an open arc of class $C^{3}$ as defined in Definition 1.5 and let

$$
\mu(x):=\mu_{e} \chi_{e}(x)+\sum_{i=1}^{n} \mu_{i} \chi_{i}(x), \quad x \in \mathbb{R}^{2} \backslash \partial D,
$$

where $\chi_{e}$ and $\chi_{i}$ are the characteristic functions of $D_{e}$ and $D_{i}$, and where $\mu_{e}>0$ and $\mu_{i}>0$ denote the magnetic permeabilities in the respective domains. The geometric setup is depicted in Figure 2.1 for the special case of $D=D_{1} \cup D_{2}$. 


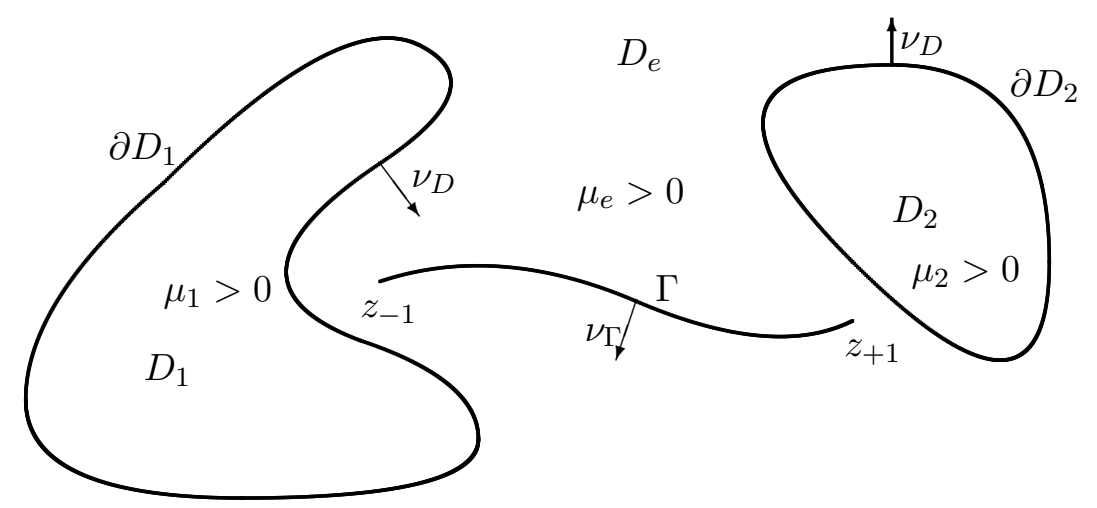

Figure 2.1: Geometric setup for Problem 2.1 for the case $D=D_{1} \cup D_{2}$.

Determine a function $u \in C^{2}\left(\mathbb{R}^{2} \backslash(\partial D \cup \Gamma)\right) \cap C\left(\mathbb{R}^{2} \backslash \partial D\right)$ satisfying the Laplace equation

$$
\Delta u=0 \quad \text { in } \mathbb{R}^{2} \backslash(\partial D \cup \Gamma)
$$

as well as the boundary conditions

$$
\begin{aligned}
u & =0 & & \text { on } \Gamma, \\
u_{+}-u_{-} & =0 & & \text { on } \partial D, \\
\frac{1}{\mu_{+}} \frac{\partial u_{+}}{\partial \nu_{D}}-\frac{1}{\mu_{-}} \frac{\partial u_{-}}{\partial \nu_{D}} & =0 & & \text { on } \partial D
\end{aligned}
$$

in the sense of uniform convergence on $\partial D$. Furthermore, for $|x| \rightarrow \infty$ it is required that

$$
u(x)-\left(\mathcal{S}^{\Gamma}\left(\frac{I}{|\Gamma|}\right)\right)(x)=\mathcal{O}(1)
$$

holds uniformly for all directions. Here $I \in \mathbb{R}$ is a given constant, $|\Gamma|$ denotes the length of $\Gamma$, and $\mathcal{S}^{\Gamma}$ is the single-layer potential as defined in Definition 1.16.

Note that $\mathcal{S}^{\Gamma}\left(\frac{I}{|\Gamma|}\right)$ is well-defined since $\frac{I}{|\Gamma|} \in C^{-\frac{1}{2}}(\Gamma)$.

\subsection{Uniqueness}

The uniqueness proof will be divided in two stages, first showing that the zero function is the only solution if the parameter $I$ is equal to zero, and secondly showing that the difference of two solutions for the same parameter $I$ yields a solution for the parameter $I=0$. 
Theorem 2.2. Let the parameter I in Problem 2.1 be equal to zero. Then each solution to Problem 2.1 vanishes identically.

Proof. Let $w$ be a solution to Problem 2.1 with parameter $I=0$, and assume that $w$ is not constant. Then there exists a disc $B \subset D \cup\left(D_{e} \backslash \Gamma\right)$ such that

$$
\int_{B}|\operatorname{grad} w|^{2} d x>0
$$

We introduce parallel curves $\partial D_{i, \pm h}:=\left\{x \pm h \nu_{D}(x): x \in \partial D_{i}\right\}$ which for sufficiently small $h>0$ are simple and of class $C^{1}$ according to Lemma 1.7. These curves form the boundaries to the corresponding domains $D_{i,-h} \subset D_{i} \subset D_{i,+h}$, and we can apply Green's first theorem (1.1) to $u=v=w$ in $D_{i,-h}$ to find

$$
\int_{D_{i,-h}}|\operatorname{grad} w|^{2} d x=\int_{\partial D_{i,-h}} w \frac{\partial w}{\partial \nu_{D}} d s
$$

We have that $\Gamma$ and all $D_{i,+h}$ are contained in a sufficiently large sphere $\Omega_{R}$, so that we can apply Theorem 1.6 to $u$ in the domain $\widetilde{\Omega}:=\Omega_{R} \backslash\left(\bigcup_{=1}^{n} D_{i,+h}\right)$ to obtain

$$
\int_{\widetilde{\Omega}}|\operatorname{grad} w|^{2} d x=\int_{\partial \Omega_{R}} w \frac{\partial w}{\partial \nu_{\Omega}} d s-\sum_{i=1}^{n} \int_{\partial D_{i,+h}} w \frac{\partial w}{\partial \nu_{D}} d s .
$$

As the parameter $I$ equals zero, condition (2.6) reduces to

$$
w(x)=\mathcal{O}(1)
$$

uniformly for all directions as $|x| \rightarrow \infty$, telling that $w$ is bounded. Now the asymptotics for $|x| \rightarrow \infty$ for bounded harmonic functions (see [47], p. 74) tell that

$$
\int_{\partial \Omega_{R}} w \frac{\partial w}{\partial \nu_{\Omega}} d s \rightarrow 0, \quad R \rightarrow \infty
$$

As $w$ and $\frac{\partial w}{\partial \nu_{D}}$ exist in the sense of uniform convergence on $\partial D_{i}$, we interchange the integration and the limit $h \searrow 0$ in (2.8) and (2.9), and use the boundary conditions (2.4) and (2.5) to obtain

$$
\int_{D_{e}}|\operatorname{grad} w|^{2} d x=-\sum_{i=1}^{n} \frac{\mu_{e}}{\mu_{i}} \int_{D_{i}}|\operatorname{grad} w|^{2} d x .
$$

Since all quotients $\frac{\mu_{e}}{\mu_{i}}$ are positive, this yields a contradiction to (2.7). Thus, $w$ is constant, and the boundary condition (2.3) implies $w \equiv 0$.

Theorem 2.3. Problem 2.1 admits at most one solution for any parameter $I \in \mathbb{R}$. 
Proof. Let $u_{1}$ and $u_{2}$ be two solutions to Problem 2.1 with parameter $I$. Then their difference $u_{1}-u_{2}$ is also an element of $C^{2}\left(\mathbb{R}^{2} \backslash(\partial D \cup \Gamma)\right) \cap C\left(\mathbb{R}^{2} \backslash \partial D\right)$ that is harmonic in $\mathbb{R}^{2} \backslash(\partial D \cup \Gamma)$ and satisfies the boundary conditions (2.3), (2.4) and (2.5) as they are homogeneous and linear in the solution. For the behaviour at infinity we calculate

$$
\left(u_{1}-u_{2}\right)-\mathcal{S}^{\Gamma}\left(\frac{0}{|\Gamma|}\right)=\left(u_{1}-\mathcal{S}^{\Gamma}\left(\frac{I}{|\Gamma|}\right)\right)-\left(u_{2}-\mathcal{S}^{\Gamma}\left(\frac{I}{|\Gamma|}\right)\right)=\mathcal{O}(1)
$$

due to the linearity of the single-layer potential operator $\mathcal{S}^{\Gamma}$ and condition (2.6) being satisfied for the solutions $u_{1}$ and $u_{2}$. This implies that $u_{1}-u_{2}$ is a solution to Problem 2.1 with parameter $I=0$, and thus vanishes identically by virtue of Theorem 2.2 , i.e. the solutions $u_{1}$ and $u_{2}$ coincide.

\section{$2.3 \quad$ Existence}

We now turn to constructing a solution for Problem 2.1 and thereby resolving the question of unique solvability. We seek a solution of Problem 2.1 by ansatz functions in the form of combined single- and double-layer potentials

$$
u(x):=\mu(x)\left[\left(\mathcal{S}^{\Gamma, I} \varphi\right)(x)+\sum_{k=1}^{n}\left(\mathcal{T}^{D_{k}} \psi_{k}\right)(x)\right], \quad x \in \mathbb{R}^{2} \backslash \partial D
$$

where $\mu$ is given by (2.1). The operators $\mathcal{T}^{D_{k}}, k=1, \ldots, n$, denote double-layer potentials as defined in Theorem 1.11 with densities $\psi_{k} \in C\left(\partial D_{k}\right)$, whereas $\mathcal{S}^{\Gamma, I}$ is a modified single-layer potential given by

$$
\left(\mathcal{S}^{\Gamma, I} \varphi\right)(x):=\mathcal{S}^{\Gamma}\left((M \varphi)+\frac{I}{|\Gamma|}\right)(x)+\frac{1}{|\Gamma|} \int_{\Gamma} \varphi d s
$$

with a density $\varphi \in C^{*}(\Gamma)$ and the operator $M$ as defined in (1.37). We are immediately able to state two theorems that are crucial to the question of unique solvability.

Theorem 2.4. Let

$$
q_{k}:=\frac{\mu_{e}-\mu_{k}}{\mu_{e}+\mu_{k}}, \quad k=1, \ldots, n,
$$

where the constants $\mu_{1}, \ldots, \mu_{n}$ and $\mu_{e}$ are given as in Problem 2.1. Then the function $u$ defined by (2.10) is a solution to Problem 2.1 provided the densities $\left(\varphi, \psi_{1}, \ldots, \psi_{n}\right)$ satisfy the following system of integral equations 


$$
\begin{aligned}
\widetilde{S}^{\Gamma} \varphi+\sum_{j=1}^{n} K_{j}^{D, \Gamma} \psi_{j} & =-S^{\Gamma}\left(\frac{I}{|\Gamma|}\right) \\
2 q_{k} \widetilde{S}_{k}^{\Gamma, D} \varphi+\psi_{k}+2 q_{k} \sum_{j=1}^{n} K_{j, k}^{D} \psi_{j} & =-2 q_{k} S_{k}^{\Gamma, D}\left(\frac{I}{|\Gamma|}\right), \quad k=1, \ldots, n .
\end{aligned}
$$

Here, the occuring integral operators are defined by

$$
\begin{array}{rlrl}
\left(K_{j, k}^{D} \psi_{j}\right)(x) & :=\int_{\partial D_{j}} \frac{\partial \Phi(x, y)}{\partial \nu(y)} \psi_{j}(y) d s(y), & x \in \partial D_{k}, \\
\left(K_{j}^{D, \Gamma} \psi_{j}\right)(x) & :=\int_{\partial D_{j}} \frac{\partial \Phi(x, y)}{\partial \nu(y)} \psi_{j}(y) d s(y), & x \in \Gamma, \\
\left(S^{\Gamma} \varphi\right)(x) & :=\int_{\Gamma} \Phi(x, y) \varphi(y) d s(y), & x \in \Gamma, \\
\left(S_{k}^{\Gamma, D} \varphi\right)(x) & :=\int_{\Gamma} \Phi(x, y) \varphi(y) d s(y), & x \in \partial D_{k}, \\
\left(\widetilde{S}^{\Gamma} \varphi\right)(x) & :=\left(S^{\Gamma}(M \varphi)\right)(x)+\frac{1}{|\Gamma|} \int_{\Gamma} \varphi d s, & & x \in \Gamma, \\
\left(\widetilde{S}_{k}^{\Gamma, D} \varphi\right)(x) & :=\left(S_{k}^{\Gamma, D}(M \varphi)\right)(x)+\frac{1}{|\Gamma|} \int_{\Gamma} \varphi d s, & & x \in \partial D_{k} .
\end{array}
$$

Proof. Let $\left(\varphi, \psi_{1}, \ldots, \psi_{n}\right)$ be a solution to (2.13) and define $u$ according to (2.10) with the parameter $I$ being taken from the right hand side of (2.13). Now the properties of the layer potentials (see Theorems 1.12 and 1.17) show that $u$ is twice continuously differentiable and harmonic in $\mathbb{R}^{2} \backslash(\Gamma \cup \partial D)$ and continuous on $\mathbb{R}^{2} \backslash \partial D$.

Considering the first equation of (2.13) we note that by adding $S^{\Gamma}\left(\frac{I}{\Gamma \mid}\right)$, the left hand side is well-defined and coincides with the restriction of $u$ to $\Gamma$. Since the right hand side vanishes, it follows that $u$ also satisfies (2.3).

Adding $2 q_{k} S^{\Gamma}\left(\frac{I}{|\Gamma|}\right)$ to the second equation of (2.13) and multiplying by $\frac{\mu_{e}+\mu_{k}}{2}$ leads to

$$
\begin{aligned}
& \mu_{e}\left[\widetilde{S}_{k}^{\Gamma, D} \varphi+S_{k}^{\Gamma, D}\left(\frac{I}{|\Gamma|}\right)+\frac{\psi_{k}}{2}+\sum_{j=1}^{n} K_{j, k}^{D} \psi_{j}\right] \\
&=\mu_{k}\left[\widetilde{S}_{k}^{\Gamma, D} \varphi+S_{k}^{\Gamma, D}\left(\frac{I}{|\Gamma|}\right)-\frac{\psi_{k}}{2}+\sum_{j=1}^{n} K_{j, k}^{D} \psi_{j}\right] \quad \text { on } \partial D_{k} .
\end{aligned}
$$


Since the single-layer potential as well as the double-layer potentials over the boundaries $\partial D_{j}$ for $j \neq k$ are continuous across $\partial D_{k}$, it follows from the jump relations (1.22) for $\mathcal{T}^{D_{k}}$ that the left-hand side of (2.20) coincides with $u$ when the boundary $\partial D_{k}$ is approached from the exterior of $D_{k}$, while the right-hand side coincides with $u$ when the boundary $\partial D_{k}$ is approached from the interior. Consequently, (2.20) implies $u_{+}=u_{-}$on $\partial D_{k}$, i.e. $u$ satisfies (2.4).

Furthermore, the single-layer potential and the double-layer potentials over the boundary contours $\partial D_{j}$ for $j \neq k$ are continuously differentiable across $\partial D_{k}$. This, together with the jump relation (1.23) for the normal derivative of the double-layer potential over $\partial D_{k}$, implies

$$
\frac{1}{\mu_{e}} \frac{\partial u_{+}}{\partial \nu_{D_{k}}}=\frac{1}{\mu_{k}} \frac{\partial u_{-}}{\partial \nu_{D_{k}}} \quad \text { on } \partial D_{k}
$$

Hence, $u$ satisfies (2.5).

Using the identity

$$
\mathcal{S}^{\Gamma, I} \varphi=\mathcal{S}^{\Gamma}\left(\frac{I}{|\Gamma|}\right)+\mathcal{S}^{\Gamma}(M \varphi)+\frac{1}{|\Gamma|} \int_{\Gamma} \varphi d s
$$

we note that the condition (2.6) at infinity reduces to

$$
\mathcal{S}^{\Gamma}(M \varphi)+\sum_{k=1}^{n} \mathcal{T}^{D_{k}} \psi_{k}=\mathcal{O}(1)
$$

which is true due to the fourth property of Theorems 1.12 and 1.17 and the fact that $\int_{\Gamma} M \varphi d s=0$. Thus, $u$ is a solution to Problem 2.1 .

Theorem 2.5. The system of integral equations given by (2.13) admits at most one solution for any $I \in \mathbb{R}$.

Proof. As the system (2.13) is linear in $\left(\varphi, \psi_{1}, \ldots, \psi_{n}\right)$, it is sufficient to prove that the homogeneous system only has the trivial solution. So let $\left(\varphi, \psi_{1}, \ldots, \psi_{n}\right)$ be a solution to (2.13) with a homogeneous right-hand side. We then note that the homogeneous right-hand side can be interpreted as the parameter $I$ being equal to zero. Now using $\left(\varphi, \psi_{1}, \ldots, \psi_{n}\right)$ and $I=0$, we define $u$ as in (2.10). Then Theorem 2.4 tells that $u$ is a solution to Problem 2.1 for $I=0$. From Theorem 2.2 it follows that $u$ vanishes identically. So we have

$$
\left[\left(\mathcal{S}^{\Gamma, I} \varphi\right)(x)+\sum_{k=1}^{n}\left(\mathcal{T}^{D_{k}} \psi_{k}\right)(x)\right] \equiv 0, \quad x \in \mathbb{R}^{2} \backslash(\Gamma \cup \partial D),
$$


as $\mu$ is positive. Thus, the interior and exterior limits on $\partial D_{k}$ vanish as well, and the jump relations (1.22) for the double-layer potential $\mathcal{T}^{D_{k}}$ lead to

$$
\begin{aligned}
\psi_{k} & =\left[\mathcal{T}^{D_{k}} \psi_{k}\right]_{+}-\left[\mathcal{T}^{D_{k}} \psi_{k}\right]_{-} \\
& =\left[\mathcal{S}^{\Gamma, I} \varphi+\sum_{j=1}^{n} \mathcal{T}^{D_{j}} \psi_{j}\right]_{+}-\left[\mathcal{S}^{\Gamma, I} \varphi+\sum_{j=1}^{n} \mathcal{T}^{D_{j}} \psi_{j}\right]_{-}=0
\end{aligned}
$$

on $\partial D_{k}$ for $k=1, \ldots, n$, since the single-layer potential over $\Gamma$ and the doublelayer potentials over $\partial D_{j}$ for $j \neq k$ are continuous across $\partial D_{k}$.

Turning to the single-layer potential we see that $u \equiv 0$ in $\mathbb{R}^{2} \backslash(\Gamma \cup \partial D)$ especially implies

$$
\frac{\partial u_{ \pm}}{\partial \nu_{\Gamma}}=0 \quad \text { on } \Gamma \backslash\left\{z_{-1}, z_{+1}\right\} .
$$

From this, using the jump relations (1.33) for the single-layer potential together with the continuous differentiability of the double-layer potentials across $\Gamma$, we conclude that

$$
\begin{aligned}
(M \varphi) & =\frac{\partial}{\partial \nu_{\Gamma}}\left[\mathcal{S}^{\Gamma}(M \varphi)\right]_{-}-\frac{\partial}{\partial \nu_{\Gamma}}\left[\mathcal{S}^{\Gamma}(M \varphi)\right]_{+} \\
& =\frac{\partial}{\partial \nu}\left[\mathcal{S}^{\Gamma, I} \varphi+\sum_{j=1}^{n} \mathcal{T}^{D_{j}} \psi_{j}\right]_{-}-\frac{\partial}{\partial \nu}\left[\mathcal{S}^{\Gamma, I} \varphi+\sum_{j=1}^{n} \mathcal{T}^{D_{j}} \psi_{j}\right]_{+}=0
\end{aligned}
$$

on $\Gamma \backslash\left\{z_{-1}, z_{+1}\right\}$, as

$$
\mathcal{S}^{\Gamma, I} \varphi=\mathcal{S}^{\Gamma}(M \varphi)+\frac{1}{|\Gamma|} \int_{\Gamma} \varphi d s
$$

for $I=0$. Having $\psi_{k}=0, k=1, \ldots, n$, and $M \varphi=0$, we now note from (2.11) and $u \equiv 0$ in $\mathbb{R}^{2} \backslash(\Gamma \cup \partial D)$ that

$$
\frac{1}{|\Gamma|} \int_{\Gamma} \varphi d s=0
$$

which implies $\varphi=0$ using $M \varphi=0$ and (1.37). Hence the only solution to the homogeneous form of (2.13) is the trivial one.

The form of the second equation of (2.13) suggests an approach via Riesz's theory for compact operators. But unfortunately, Riesz's theory is not directly applicable to (2.13) in its present form as the first equation appears to be an operator equation of the first kind. Instead, we will proceed by splitting the operator $\widetilde{S}^{\Gamma}$ into an invertible and a compact part through parametrizing $\Gamma$ and, subsequently, introducing a cosine substitution as suggested by Multhopp [52] in the form applied by Kress [46] to the Dirichlet scattering problem for the open arc. 
Executing the parametrization over $\Gamma$ and substituting the cosine in both the image space and pre-image space of the first equation of (2.13) yields the identity

$$
\left(\widetilde{S}^{\Gamma} \varphi\right)(\gamma(\cos t))=(L \widetilde{\varphi})(t)+\left(L_{0} \widetilde{\varphi}\right)(t),
$$

where the operator $L$ for $t \in[0, \pi]$ is defined by

$$
(L \widetilde{\varphi})(t):=\frac{1}{4 \pi} \int_{0}^{\pi}\left\{-\ln \left[4(\cos t-\cos \tau)^{2}\right]+4\right\} \widetilde{\varphi}(\tau) d \tau
$$

and the operator $L_{0}$ is given through

$$
\left(L_{0} \widetilde{\varphi}\right)(t):=\frac{1}{4 \pi} \int_{0}^{\pi}\left\{k_{0}(t, \tau)-4\right\} \widetilde{\varphi}(\tau) d \tau, \quad t \in[0, \pi] .
$$

Here, the integral kernel $k_{0}$ is given by

$$
\begin{aligned}
k_{0}(t, \tau):= & \ln \frac{4(\cos t-\cos \tau)^{2}}{|\gamma(\cos t)-\gamma(\cos \tau)|^{2}}+\frac{4 \pi}{|\Gamma|} \\
& -\frac{1}{|\Gamma|} \int_{0}^{\pi} \ln \left(\frac{1}{|\gamma(\cos t)-\gamma(\cos \sigma)|^{2}}\right)\left|\gamma^{\prime}(\cos \sigma)\right| \sin \sigma d \sigma
\end{aligned}
$$

for $t, \tau \in \mathbb{R}$ with $t \neq \tau$, and we have set

$$
\widetilde{\varphi}(\tau):=\varphi(\gamma(\cos \tau))\left|\gamma^{\prime}(\cos \tau)\right| \sin \tau
$$

for the density.

Similarly, by introducing the parametrization for $\Gamma$ and the cosine substitution in the second equation of (2.13) we obtain

$$
\left(\widetilde{S}_{i}^{\Gamma, D} \varphi\right)(x)=\left(\widetilde{K}_{i}^{\Gamma, D} \widetilde{\varphi}\right)(x)
$$

for $i=1, \ldots, n$, where the operators $\widetilde{K}_{i}^{\Gamma, D}$ are defined as

$$
\left(\widetilde{K}_{i}^{\Gamma, D} \widetilde{\varphi}\right)(x):=\frac{1}{2 \pi} \int_{0}^{\pi} \widetilde{k}_{i}^{\Gamma, D}(x, \tau) \widetilde{\varphi}(\tau) d \tau, \quad x \in \partial D_{i},
$$

with integral kernel $\widetilde{k}_{i}^{\Gamma, D}$ given by

$$
\begin{aligned}
\widetilde{k}_{i}^{\Gamma, D}(x, \tau):= & \ln \frac{1}{|x-\gamma(\cos \tau)|}+\frac{2 \pi}{|\Gamma|} \\
& -\frac{1}{|\Gamma|} \int_{0}^{\pi} \ln \left(\frac{1}{|x-\gamma(\cos \sigma)|}\right)\left|x^{\prime}(\cos \sigma)\right| \sin \sigma d \sigma
\end{aligned}
$$

for $x \in \partial D_{i}$ and $\tau \in \mathbb{R}$ and the density given as in (2.24). 
Notice that in both cases checking the equalities (2.21) and (2.25) is done by re-arranging (2.18) and (2.19) such that the density $\varphi$ only occurs in the outer integration. In the case of (2.21) we additionally have added and substracted the kernel of $L$ and split the resulting expression suitably.

Parametrizing and substituting likewise in the other expressions of the first equation of (2.13) we obtain the system

$$
\begin{aligned}
\left(L+L_{0}\right) \widetilde{\varphi}+\sum_{j=1}^{n} \widetilde{K}_{j}^{D, \Gamma} \psi_{j} & =-\widehat{S}^{\Gamma}\left(\frac{I}{|\Gamma|}\right), \\
2 q_{k} \widetilde{K}_{k}^{\Gamma, D} \widetilde{\varphi}+\psi_{k}+2 q_{k} \sum_{j=1}^{n} K_{j, k}^{D} \psi_{j} & =-2 q_{k} S_{k}^{\Gamma, D}\left(\frac{I}{|\Gamma|}\right),
\end{aligned}
$$

where for $t \in[0, \pi]$ and $j=1, \ldots, n$, the operators $\widetilde{K}_{j}^{D, \Gamma}$ and $\widehat{S}^{\Gamma}$ are defined as

$$
\left(\widetilde{K}_{j}^{D, \Gamma} \psi_{j}\right)(t):=\int_{\partial D_{j}} \frac{\partial \Phi(\gamma(\cos t), y)}{\partial \nu(y)} \psi_{j}(y) d s(y)
$$

and, respectively,

$$
\left(\widehat{S}^{\Gamma}\left(\frac{I}{|\Gamma|}\right)\right)(t):=\left(S^{\Gamma}\left(\frac{I}{|\Gamma|}\right)\right)(\gamma(\cos t))
$$

To simplify the notation we can re-write (2.28) in matrix notation introducing

$$
\begin{gathered}
S:=\left(\begin{array}{cccc}
L & 0 & \cdots & 0 \\
0 & I d & \cdots & 0 \\
\vdots & \vdots & \ddots & \vdots \\
0 & 0 & \cdots & I d
\end{array}\right), \\
A:=\left(\begin{array}{cccc}
L_{0} & \widetilde{K}_{1}^{D, \Gamma} & \cdots & \widetilde{K}_{n}^{D, \Gamma} \\
2 q_{1} \widetilde{K}_{1}^{\Gamma, D} & 2 q_{1} K_{1,1}^{D} & \cdots & 2 q_{1} K_{n, 1}^{D} \\
\vdots & \vdots & \ddots & \vdots \\
2 q_{n} \widetilde{K}_{n}^{\Gamma, D} & 2 q_{n} K_{1, n}^{D} & \cdots & 2 q_{n} K_{n, n}^{D}
\end{array}\right),
\end{gathered}
$$

for the operators of the left-hand side, and

$$
f:=\left(\begin{array}{llll}
-\widehat{S}^{\Gamma}\left(\frac{I}{|\Gamma|}\right) & -2 q_{1} S_{1}^{\Gamma, D}\left(\frac{I}{|\Gamma|}\right) & \cdots & -2 q_{n} S_{n}^{\Gamma, D}\left(\frac{I}{|\Gamma|}\right)
\end{array}\right)^{T}
$$

for the right-hand side. Accordingly, we introduce the product spaces

$$
W_{0}:=C_{2 \pi, e}^{0, \alpha} \times C\left(\partial D_{1}\right) \times \ldots \times C\left(\partial D_{n}\right)
$$


and

$$
W_{1}:=C_{2 \pi, e}^{1, \alpha} \times C\left(\partial D_{1}\right) \times \ldots \times C\left(\partial D_{n}\right),
$$

finding that (2.28) now reads

$$
(S+A) \Psi=f
$$

where we have set $\Psi:=\left(\widetilde{\varphi}, \psi_{1}, \ldots, \psi_{n}\right) \in W_{0}$.

Lemma 2.6. For each solution $\left(\widetilde{\varphi}, \psi_{1}, \ldots, \psi_{n}\right) \in W_{0}$ of (2.36) the corresponding tupel $\left(\varphi, \psi_{1}, \ldots, \psi_{n}\right)$ with $\varphi \in C^{*}(\Gamma)$ given through

$$
\varphi(x):=\frac{\widetilde{\varphi}(\arccos t)}{\left|\gamma^{\prime}(t)\right| \sqrt{1-t^{2}}}, \quad x=\gamma(t), t \in(-1,1)
$$

is a solution to (2.13). Furthermore, if a solution to (2.36) exists, it is uniquely determined.

Proof. Let $\left(\widetilde{\varphi}, \psi_{1}, \ldots, \psi_{n}\right)$ be a solution to (2.36). Then $\varphi$ defined by (2.37) is an element of $C^{*}(\Gamma)$ since the elements of $C^{*}(\Gamma)$ are characterized exactly by $(2.37)$ indepedently of the parametrization $\gamma$. Performing the parametrization of $\Gamma$ and the cosine substitution as above, we note that we are left to prove that indeed

$$
\widetilde{\varphi}(\tau)=\varphi(\gamma(\cos \tau))\left|\gamma^{\prime}(\cos \tau)\right| \sin \tau
$$

which is immediately true by inserting the definition (2.37) for $\varphi$ into (2.24) as the square root term cancels against the sine term and the norms also cancel. The uniqueness claim now is a consequence of the definition of $\varphi$ in combination with Theorem 2.5.

Lemma 2.6 relates a solution of (2.36) to the densities of the ansatz function given in (2.10). With this relation in hand we can now analyze (2.36) in the scope of Riesz's theory for compact operators. We prepare the central result of this chapter by examining the mapping properties of the operators $S$ and $A$. Furthermore, we will show that the right-hand side $f$ belongs to $W_{1}$.

Lemma 2.7. The operator $S$ given by (2.31) is invertible from $W_{0}$ to $W_{1}$.

Proof. We observe that $S$ is a diagonal operator with the entries being either the operator $L$, which is invertible from $C_{2 \pi, e}^{0, \alpha}$ to $C_{2 \pi, e}^{1, \alpha}$ due to Corollary 1.20, or the identity on $C\left(\partial D_{k}\right)$ for $k=1, \ldots, n$. Hence, $S$ is invertible from $W_{0}$ to $W_{1}$. 
Lemma 2.8. The operator A given by (2.32) is a compact operator mapping from $W_{0}$ to $W_{1}$.

Proof. For detailed proofs of the mapping properties of the diagonal operators we refer to [36]. There it has been shown that the operators $K_{i, i}^{D}$ have continuous kernels, and are hence compact operators from $C\left(\partial D_{i}\right)$ to $C\left(\partial D_{i}\right)$. It has been shown furthermore that the kernel of the operator $L_{0}$ can be extended to an even, $2 \pi$-periodic, twice continuously differentiable function on $\mathbb{R}^{2}$. Consequently $L_{0}$ is a bounded operator from $C_{2 \pi, e}^{0, \alpha}$ to $C_{2 \pi, e}^{2}$, and thus compact as an operator mapping to $C_{2 \pi, e}^{1, \alpha}$ by compact embedding.

For the off-diagonal operators $K_{i, j}^{D}, i \neq j$, we observe that they are restrictions of the double-layer potential operators $\mathcal{T}_{i}{ }^{D}$ to the $C^{2}$-smooth boundaries $\partial D_{j}$ that do not intersect with their respective domain of integration. So it can be seen from (2.14) that the corresponding kernels are continuous. Consequently, the operators $K_{i, j}^{D}$ are compact mappings from $C\left(\partial D_{i}\right)$ to $C\left(\partial D_{j}\right)$.

Similarly, the operators $K_{i}^{D, \Gamma}$ are restrictions of the double-layer potential operators $\mathcal{T}_{i}{ }^{D}$ to $\Gamma$. Hence, the kernels of these operators are non-singular. In particular they are twice continuously differentiable on $\Gamma$. As the operators $\widetilde{K}_{i}^{D, \Gamma}$ are obtained from the operators $K_{i}^{D, \Gamma}$ by substituting the twice-differentiable, $2 \pi$-periodic and even function $\gamma(\cos t)$ in the image domain $\Gamma$, they are bounded operators from $C\left(\partial D_{i}\right)$ to $C_{2 \pi, e}^{2}$. The compactness of the operators $\widetilde{K}_{i}^{D, \Gamma}$ as operators from $C\left(\partial D_{i}\right)$ to $C_{2 \pi, e}^{1, \alpha}$ now follows by compact embedding.

Finally, for the operators $\widetilde{K}_{i}^{\Gamma, D}$ it can be seen from (2.27) that the kernels are continuous on $\partial D_{i} \times \mathbb{R}$ as $\Gamma$ and $\partial D_{i}$ are disjoint for any $i=1, \ldots, n$. Consequently, the operators $\widetilde{K}_{i}^{\Gamma, D}$ are compact from $C_{2 \pi, e}^{1, \alpha}$ to $C\left(\partial D_{i}\right)$.

Now $A$ itself is a compact operator from $W_{0}$ to $W_{1}$ as it is the sum of compact operators in each of its components.

Lemma 2.9. The function $f$ given by (2.33) is an element of $W_{1}$.

Proof. Let us consider the first component of $f$. It can be seen from (2.30) that the operator $\widehat{S}$ is mapping to $2 \pi$-periodic and even functions. Furthermore, we know from [47], Theorem 7.28, that the single-layer potential is uniformly Hölder continuously differentiable on its domain of integration provided the density is itself uniformly Hölder continuous. As this holds true for the constant $\frac{I}{|\Gamma|}$, it follows that $-\widehat{S}^{\Gamma}\left(\frac{I}{|\Gamma|}\right) \in C_{2 \pi, e}^{1, \alpha}$. For the other components of $f$ we observe that the operators $S_{i}^{\Gamma, D}$ are the restricions of the single-layer potential operator $\mathcal{S}^{\Gamma}$ to the $C^{2}$-smooth boundary contours $\partial D_{i}$. Hence, $-2 q_{i} S_{i}^{\Gamma, D}\left(\frac{I}{|\Gamma|}\right)$ is a continuous function due to Theorem 1.17, and thus $f$ is an element of $W_{1}$. 
With these properties for the operators and the right-hand side of (2.36) in hand, we can now formulate and prove the main result of the chapter - the unique solvability of the Boundary Value Problem 2.1.

Theorem 2.10. Problem 2.1 is uniquely solvable for any parameter $I \in \mathbb{R}$.

Proof. Riesz's theory for compact operators establishes bijectivity for operator equations of the form of (2.36), provided $S$ is boundedly invertible, $A$ is compact, and the operator is injective.

Now the results of Lemma 2.7, Lemma 2.8 and Lemma 2.9 state that (2.36) holds in $W_{1}$, that $S$ is indeed invertible, and $A$ is indeed compact. Furthermore, the operator $S+A$ has a trivial nullspace due to Lemma 2.6, and thus we obtain from Riesz's theory that $S+A$ is also surjective, which means that (2.36) has a solution for each right hand side $f \in W_{1}$. Now by virtue of Lemma 2.6 and Theorem 2.4, the corresponding potential $u$ defined by (2.10) is a solution to Problem 2.1 with parameter $I$. The uniqueness is due to Theorem 2.3.

\subsection{Properties of the solution to the Boundary Value Problem}

In the final section of this chapter we will examine certain properties of the solution to Problem 2.1 that are motivated from the physical situation described in the introduction. The main objective here is to identify and characterize the jump

$$
\left[\frac{\partial u}{\partial \nu_{\Gamma}}\right]:=\frac{\partial u_{-}}{\partial \nu_{\Gamma}}-\frac{\partial u_{+}}{\partial \nu_{\Gamma}}
$$

of the normal derivative of the solution $u$ to Problem 2.1 across $\Gamma$ as a distributed electric current that is penetrating $\Gamma$ in positive $x_{3}$-direction, if we think of Problem 2.1 embedded into $\mathbb{R}^{3}$.

Definition 2.11. Let $\Gamma \subset \mathbb{R}^{2}$ be an open arc of class $C^{3}$. A continuous function $j: \Gamma \backslash\left\{z_{-1}, z_{+1}\right\} \rightarrow \mathbb{R}$ is called current distribution for $\Gamma$, if it satisfies

$$
\int_{\Gamma} j d s=I,
$$

where $I \in \mathbb{R}$ is the total current. 
From an Ampèrian viewpoint a current is not penetrating the entire of a volume sample as a scalar quantity, but it is regarded as a vectorial quantity that depends on the position within the sample. Consequently, the current $I_{\Sigma}$ penetrating a particular cross-section $\Sigma$ is given by

$$
I_{\Sigma}:=\int_{\Sigma}\left\langle\nu_{\Sigma}, \mathbf{j}(x)\right\rangle d x
$$

where $\nu_{\Sigma}$ is the surface normal to $\Sigma$ and $\mathbf{j}$ is the vector-valued current distribution. The modelling assumptions from the introduction tell that in the case discussed, $\mathbf{j}$ is assumed to be given as $j_{0} \nu_{\Sigma}$ for some scalar distribution $j_{0}: \Sigma \rightarrow \mathbb{R}$. Furthermore, the surface $\Sigma$ is assumed to have height $\delta<<W$, where $W$ is the width, and $j_{0}$ is assumed to be constant along the height of $\Sigma$. So introducing local coordinates $x=(u, v)$ such that $x \in \Sigma$ implies $|v| \leq \delta$ and defining

$$
j(u):=2 \delta j_{0}(u, 0)=\int_{-\delta}^{\delta} j_{0}(u, v) d s(v)
$$

we find

$$
\int j(u) d s(u)=\int_{\Sigma} j_{0}(x) d x=\int_{\Sigma}\left\langle\nu_{\Sigma}, \mathbf{j}(x)\right\rangle d x
$$

which renders Definition 2.11 sensible.

We will see in the next lemma that the jump (2.38) is indeed a current distribution in the sense of Definition 2.11.

Lemma 2.12. Let $\Gamma \subset \mathbb{R}^{2}$ be an open arc of class $C^{3}$ with endpoints $z_{ \pm 1}$. Then the solution $u$ to Problem 2.1 with parameter I satisfies

$$
\int_{\Gamma}\left[\frac{\partial u}{\partial \nu}\right] d s=I
$$

i.e. $\left[\frac{\partial u}{\partial \nu}\right]$ is a current distribution in the sense of Definition 2.11. Furthermore, we have that $\left[\frac{\partial u}{\partial \nu}\right] \in C^{*}(\Gamma)$.

Proof. Let $u$ be the solution to Problem 2.1 with parameter $I$. Then we know from Section 2.3 that $u$ can be represented in the form of (2.10). Hence, the normal derivatives on $\Gamma$ are given by

$$
\frac{\partial u_{ \pm}}{\partial \nu_{\Gamma}}=\frac{\partial}{\partial \nu_{\Gamma}}\left(\mathcal{S}^{\Gamma}\left(M \varphi+\frac{I}{|\Gamma|}\right)\right)_{ \pm}+\sum_{k=1}^{n} \frac{\partial}{\partial \nu_{\Gamma}}\left(\mathcal{T}^{D_{k}} \psi_{k}\right) .
$$

As the double-layer potentials are continuously differentiable across $\Gamma$ we obtain for the jump

$$
\begin{aligned}
{\left[\frac{\partial u}{\partial \nu_{\Gamma}}\right](x) } & =\frac{\partial}{\partial \nu_{\Gamma}}\left(\mathcal{S}^{\Gamma}\left(M \varphi+\frac{I}{|\Gamma|}\right)\right)_{-}(x)-\frac{\partial}{\partial \nu_{\Gamma}}\left(\mathcal{S}^{\Gamma}\left(M \varphi+\frac{I}{|\Gamma|}\right)\right)_{+}(x) \\
& =(M \varphi)(x)+\frac{I}{|\Gamma|}
\end{aligned}
$$


for $x \in \Gamma \backslash\left\{z_{-1}, z_{+1}\right\}$ using the jump relation (1.33) with the density $\varphi \in C^{*}(\Gamma)$. This implies that $\left[\frac{\partial u}{\partial \nu_{\Gamma}}\right]$ is given as the sum of $\varphi \in C^{*}(\Gamma)$ and a constant. Now the constants are contained in $C^{*}(\Gamma)$ as

$$
\left|\gamma^{\prime}(\cos t) \sin t\right|, \quad t \in[0, \pi]
$$

has an extension in $C_{2 \pi, e}^{0, \alpha}$ according to Lemma 3.8, and thus $\left[\frac{\partial u}{\partial \nu_{\Gamma}}\right] \in C^{*}(\Gamma)$. Finally, with the aid of Lemma 1.15 we see that

$$
\begin{aligned}
\int_{\Gamma}\left[\frac{\partial u}{\partial \nu_{\Gamma}}\right] d s & =\int_{\Gamma}(M \varphi)+\frac{I}{|\Gamma|} d s \\
& =\int_{\Gamma} \varphi d s-\frac{1}{|\Gamma|} \int_{\Gamma} \varphi d s \int_{\Gamma} 1 d s+\frac{I}{|\Gamma|} \int_{\Gamma} 1 d s=I .
\end{aligned}
$$

In combination with Definition 2.11, Lemma 2.12 has given a physical meaning to the jump (2.38) of the solution to Problem 2.1, but it has by no means characterized the jump. A cursory glance on the form of (2.38), that has been deduced as

$$
\left[\frac{\partial u}{\partial \nu_{\Gamma}}\right]=\varphi-\frac{1}{|\Gamma|} \int_{\Gamma} \varphi d s+\frac{I}{|\Gamma|}
$$

in the proof of Lemma 2.12, might give rise to the claim that the current distribution develops singularities of square root type at the endpoints of $\Gamma$ since the characterization of $\varphi \in C^{*}(\Gamma)$ contains the factor $\left(1-t^{2}\right)^{-\frac{1}{2}}$. This disregards the influence of the associated function $\widetilde{\varphi} \in C_{2 \pi, e}^{0, \alpha}$ in a dangerous fashion. According to Lemma 1.15 the behaviour of $\widetilde{\varphi}$ near $t=0$ and $t=\pi$ does not only determine whether $\varphi$ is square-integrable or not, but $\widetilde{\varphi}$ also determines whether $\varphi$ is bounded or not. In particular, if the zero of $\widetilde{\varphi}$ at $t=0$ or $t=\pi$ is stronger than order $\frac{1}{2}$ (it is of order $\alpha$ anyway, since $\widetilde{\varphi} \in C_{2 \pi, e}^{0, \alpha}$ ), it is stronger than the singularity. Thus, it yields a function $\varphi \in C^{*}(\Gamma)$ that is bounded in a neighbourhood of that particular endpoint.

In the remainder of this section we will therefore investigate into the behaviour of the current distribution (2.38) near the endpoints $z_{ \pm 1}$ of $\Gamma$. Our discussion is mainly based on the book by Muskelishvili [53]. The ideas presented there have been used in the context of boundary integral equations over open arcs by Hayashi in [34], where a full theory for the direct scattering problem for open arcs has been developed.

We will first derive a characterization of the behaviour of a solution to a Cauchy type integral equation over a union of open arcs near their endpoints. Secondly, we will apply this result to the solution of Problem 2.1 by identifying the current distribution (2.38) as the solution to a Cauchy type integral equation over $\Gamma$. 
Theorem 2.13. Let $L=\bigcup_{i=1}^{n} L_{i}$ be the union of non-intersecting, disjoint, regular, smooth, open arcs with endpoints $\left(z_{ \pm i}\right), i=1, \ldots, n$. Let $f \in C^{0, \alpha}(L)$, and suppose that there exists a solution $\varphi$ to

$$
\int_{L} \frac{\varphi(\zeta)}{\zeta-z} d \zeta=f(z), \quad z \in L
$$

Then $\varphi$ is given by

$$
\varphi(z)=\frac{1}{i \pi} \frac{\sqrt{R_{1}(z)}}{\sqrt{R_{2}(z)}} \int_{L} \frac{f(\zeta)}{\zeta-z} \frac{\sqrt{R_{2}(\zeta)}}{\sqrt{R_{1}(\zeta)}} d \zeta+\frac{P_{n-1}(z)}{\sqrt{R_{1}(z) R_{2}(z)}}, \quad z \in L
$$

where $P_{n-1}$ is a polynomial of degree $n-1$ and

$$
R_{1 / 2}(z)=\prod_{j \in I_{1 / 2}}\left(z-z_{j}\right), \quad z \in L
$$

Here, $I_{1}$ is the set of all endpoints where $\varphi$ is bounded, whereas $I_{2}$ is the set of all endpoints where $\varphi$ is unbounded.

Proof. see [53], p. 251.

We remark here that the formulation of Theorem 2.13 supposes the existence of a solution to (2.41). This implies in particular that the right-hand side $f$ satisfies certain solvability conditions in the case where $\left|I_{1}\right| \geq n$. From this explicit inversion formula we can now deduce the behaviour of the solution $\varphi$ to (2.41) near the endpoints $z_{ \pm i}$ of $L$.

Corollary 2.14. Under the assumptions of Theorem 2.13 let $\varphi$ be a solution to equation (2.41). Then the following holds:

1. Let $z_{j}$ be an endpoint, where $\varphi$ is bounded. Then

$$
\varphi\left(z_{j}\right)=0
$$

2. Let $z_{j}$ be an endpoint, where $\varphi$ is unbounded. Then for a neighbourhood $U$ of $z_{j}$

$$
\varphi(z)=\left(z-z_{j}\right)^{-\frac{1}{2}} \varphi_{0}(z), \quad z \in U \cap L,
$$

where $\varphi_{0}$ is bounded in $U \cap L$. 
Proof. We begin with the first statement. So let $z_{j}$ be an endpoint, where $\varphi$ is bounded. It follows from [53], $\S 29$, that near $z_{j}$ we have

$$
\int_{L} \frac{f(\zeta)}{\zeta-z} \frac{\sqrt{R_{2}(\zeta)}}{\sqrt{R_{1}(\zeta)}} d \zeta=f_{0}(z)\left(z-z_{j}\right)^{-\alpha_{0}}
$$

where $\alpha_{0}<\frac{1}{2}$ and $f_{0}$ is bounded in a neighbourhood of $z_{j}$, yielding

$$
\frac{1}{i \pi} \frac{\sqrt{R_{1}(z)}}{\sqrt{R_{2}(z)}} \int_{L} \frac{f(\zeta)}{\zeta-z} \frac{\sqrt{R_{2}(\zeta)}}{\sqrt{R_{1}(\zeta)}} d \zeta=\left(z-z_{j}\right)^{\frac{1}{2}-\alpha_{0}} \frac{f_{0}(z) \sqrt{\widetilde{R}_{1}(z)}}{i \pi \sqrt{R_{2}(z)}},
$$

where $R_{1}$ and $\widetilde{R}_{1}$ are related by $R_{1}(z)=\widetilde{R}_{1}(z)\left(z-z_{j}\right)$. Thus, we observe by re-arranging (2.42) and plugging in the above that

$$
\frac{P_{n-1}(z)}{\sqrt{R_{1}(z) R_{2}(z)}}=\varphi(z)-\left(z-z_{j}\right)^{\frac{1}{2}-\alpha_{0}} \frac{f_{0}(z) \sqrt{\widetilde{R}_{1}(z)}}{i \pi \sqrt{R_{2}(z)}}
$$

is also bounded in a neighbourhood of $z_{j}$, which implies that $P$ vanishes at $z_{j}$. Since $P$ is a polynomial, it has to contain the factor $\left(z-z_{j}\right)$, and $z_{j}$ being arbitrary yields

$$
\varphi(z)=\frac{1}{i \pi} \frac{\sqrt{R_{1}(z)}}{\sqrt{R_{2}(z)}} \int_{L} \frac{f(\zeta)}{\zeta-z} \frac{\sqrt{R_{2}(\zeta)}}{\sqrt{R_{1}(\zeta)}} d \zeta+\frac{\sqrt{R_{1}(z)}}{\sqrt{R_{2}(z)}} Q_{n-p-1}(z), \quad z \in L,
$$

where $p=\left|I_{1}\right|$ and $Q_{n-p-1}$ satisfies $P_{n-1}=Q_{n-p-1} R_{1}$. In the case, where $p \geq n$, we have $Q_{n-p-1} \equiv 0$. Now we notice from (2.43) and (2.44) that

$$
\varphi\left(z_{j}\right)=0, \quad \text { for all } j \in I_{1} .
$$

For the second statement, let $z_{j}$ be an endpoint, where $\varphi$ is unbounded. Reordering the right-hand side of (2.44) leads to

$$
\varphi(z)=\frac{1}{\sqrt{R_{2}(z)}}\left(\frac{\sqrt{R_{1}(z)}}{i \pi} \int_{L} \frac{f(\zeta)}{\zeta-z} \frac{\sqrt{R_{2}(\zeta)}}{\sqrt{R_{1}(\zeta)}} d \zeta+\sqrt{R_{1}(z)} Q_{n-p-1}(z)\right)
$$

near $z_{j}$. Again, from [53], §29, it follows that

$$
\int_{L} \frac{f(\zeta)}{\zeta-z} \frac{\sqrt{R_{2}(\zeta)}}{\sqrt{R_{1}(\zeta)}} d \zeta
$$

is bounded, from which we conclude that

$$
\varphi_{0}(z):=\frac{\sqrt{R_{1}(z)}}{i \pi} \int_{L} \frac{f(\zeta)}{\zeta-z} \frac{\sqrt{R_{2}(\zeta)}}{\sqrt{R_{1}(\zeta)}} d \zeta+\sqrt{R_{1}(z)} Q_{n-p-1}(z), \quad z \in L,
$$

is also bounded near $z_{j}$ for all $z_{j} \in I_{2}$. 
Now it remains to identify the jump (2.38) as the solution to an equation of the form of (2.41), which will be done in the following Theorem.

Theorem 2.15. Let $I \in \mathbb{R} \backslash\{0\}$, and consider a solution $u$ to Problem 2.1 for the parameter I. Then the behaviour of the jump $\left[\frac{\partial u}{\partial \nu_{\Gamma}}\right]$ of the normal derivative of $u$ across $\Gamma$ near an endpoint $z_{i} \in\left\{z_{-1}, z_{+1}\right\}$ of $\Gamma$ is characterized as follows:

i) Either $\left[\frac{\partial u}{\partial \nu_{\Gamma}}\right]$ is bounded in a neighbourhood of $z_{i}$. Then we have that

$$
\left[\frac{\partial u}{\partial \nu_{\Gamma}}\right]\left(z_{i}\right)=0
$$

ii) $\operatorname{Or}\left[\frac{\partial u}{\partial \nu_{\Gamma}}\right]$ is unbounded in a neighbourhood of $z_{i}$. Then the singularity is of the form

$$
\left[\frac{\partial u}{\partial \nu_{\Gamma}}\right](x)=\left(\left|x-z_{i}\right|\right)^{-\frac{1}{2}} f_{i}^{*}(x), \quad \Gamma \ni x \rightarrow z_{i},
$$

where the functions $f_{i}^{*} \in C(\Gamma), i= \pm 1$, are bounded in a neighbourhood of $z_{i}$.

Proof. The proof is organized as follows. Firstly, we identify $\left[\frac{\partial u}{\partial \nu_{\Gamma}}\right]$ as the solution to a first kind integral equation. Secondly, we show that this integral equation can be transformed to a complex integral equation of Cauchy type. Thirdly, we will verify that the obtained Cauchy type integral equation has a Hölder continuous right-hand side. Finally, we will derive the statement from Theorem 2.13 and Corollary 2.14.

Step 1: Let $u$ be the solution to Problem 2.1 for the parameter $I$. Then we know that $u$ can be represented by (2.10) and satisfies the homogeneous Dirichlet condition (2.3) on $\Gamma$. Using the representation (2.40), a comparison with (2.10) reveals that the jump is a solution to

$$
\int_{\Gamma} \Phi(x, y)\left[\frac{\partial u}{\partial \nu_{\Gamma}}\right](y) d s(y)=-\frac{1}{|\Gamma|} \int_{\Gamma} \varphi d s-\int_{\partial D} \frac{\partial \Phi(x, y)}{\partial \nu_{D}(y)} \psi(y) d s(y),
$$

which holds for $x \in \Gamma$. Note that in order to keep the notation as simple as possible, we have written the double-layer potential over the boundary $\partial D$, which may consist of several connected components. The density $\psi$ also has to be understood in this sense.

The right-hand side of (2.47) is three times continuously differentiable along $\Gamma$, since $\Gamma$ is of class $C^{3}$ and the functions are analytic in a neighbourhood of $\Gamma$. Consequently, the left-hand side of (2.47) is also differentiable and due to (2.40) 
and $\varphi$ being an element of $C^{*}(\Gamma)$, we can carry out the differentiation under the integral (see [47], Theorem 7.28). Now differentiation of (2.47) yields

$$
\int_{\Gamma} \frac{\partial \Phi(x, y)}{\partial t_{\Gamma}(x)}\left[\frac{\partial u}{\partial \nu_{\Gamma}}\right](y) d s(y)=-\frac{\partial}{\partial t_{\Gamma}(x)} \int_{\partial D} \frac{\partial \Phi(x, y)}{\partial \nu_{D}(y)} \psi(y) d s(y)
$$

for $x \in \Gamma$, which completes the first step.

Step 2: Now let $\widetilde{\Gamma}:=\Gamma \cup \Gamma^{c}$ be a closed, simple, piecewise smooth curve of length $2 L>|\Gamma|$ which we parametrize by arclength, i.e. there exists $\gamma:[0,2 L] \rightarrow \mathbb{R}^{2}$ such that

$$
\begin{aligned}
\Gamma & =\{\gamma(t): t \in[0,|\Gamma|]\}, \\
\Gamma^{c} & =\{\gamma(t): t \in[|\Gamma|, 2 L]\} \\
\left\|\gamma^{\prime}(t)\right\| & =1, \quad t \in[0,2 L] .
\end{aligned}
$$

Introducing complex variables $z$ and $\zeta$, we define

$$
\Sigma:=\left\{z=e^{\frac{i \pi s}{L}}: s \in[0,|\Gamma|]\right\}
$$

as well as

$$
\begin{aligned}
\widetilde{\varphi}(\zeta)=\widetilde{\varphi}\left(e^{\frac{i \pi \tau}{L}}\right) & :=\left[\frac{\partial u}{\partial \nu_{\Gamma}}\right](\gamma(\tau)), \quad \tau \in(0,|\Gamma|), \\
h(z)=h\left(e^{\frac{i \pi s}{L}}\right) & :=\frac{\partial}{\partial t_{\Gamma}(\gamma(s))} \int_{\partial D} \frac{\partial \Phi(\gamma(s), y)}{\partial \nu_{D}(y)} \psi(y) d s(y), \quad s \in[0,|\Gamma|], \\
k(z, \zeta)=k\left(e^{\frac{i \pi s}{L}}, e^{\frac{i \pi \tau}{L}}\right) & :=\frac{L}{i \pi} \frac{\partial \Phi(\gamma(s), \gamma(\tau))}{\partial t_{\Gamma}(\gamma(s))} e^{-\frac{i \pi \tau}{L}}+\frac{1}{2 \pi} \frac{1}{e^{\frac{i \pi \tau}{L}}-e^{\frac{i \pi s}{L}}}
\end{aligned}
$$

for $s, \tau \in[0,|\Gamma|], s \neq \tau$. Then, by parametrizing (2.48) and adding

$$
\frac{i}{2 L} \int_{0}^{|\Gamma|} \frac{e^{\frac{i \pi \tau}{L}}}{e^{\frac{i \pi \tau}{L}}-e^{\frac{i \pi s}{L}}}\left[\frac{\partial u}{\partial \nu_{\Gamma}}\right](\gamma(\tau)) d \tau
$$

to both sides, we obtain

$$
\begin{aligned}
& \frac{1}{2 \pi} \int_{0}^{|\Gamma|} \frac{\left[\frac{\partial u}{\partial \nu_{\Gamma}}\right](\gamma(\tau))}{e^{\frac{i \pi \tau}{L}}-e^{\frac{i \pi s}{L}}} \frac{i \pi}{L} e^{\frac{i \pi \tau}{L}} d \tau-\frac{\partial}{\partial t_{\Gamma}(\gamma(s))} \int_{\partial D} \frac{\partial \Phi(\gamma(s), y)}{\partial \nu_{D}(y)} \psi(y) d s(y) \\
& =\int_{0}^{|\Gamma|}\left(\frac{L}{i \pi} \frac{\partial \Phi(\gamma(s), \gamma(\tau))}{\partial t_{\Gamma}(\gamma(s))} e^{-\frac{i \pi \tau}{L}}+\frac{1}{2 \pi} \frac{1}{e^{\frac{i \pi \tau}{L}}-e^{\frac{i \pi s}{L}}}\right)\left[\frac{\partial u}{\partial \nu_{\Gamma}}\right](\gamma(\tau)) \frac{i \pi}{L} e^{\frac{i \pi \tau}{L}} d \tau .
\end{aligned}
$$

Employing the complex variables $z$ and $\zeta$, bringing $h(\gamma(s))$ to the other side and multiplying by $-2 i$ leads to

$$
\frac{1}{i \pi} \int_{\Sigma} \frac{\widetilde{\varphi}(\zeta)}{\zeta-z} d \zeta=-2 i h(z)-2 i \int_{\Sigma} k(z, \zeta) \widetilde{\varphi}(\zeta) d \zeta, \quad z \in \Sigma
$$


which implies that we have transformed (2.48) into an integral equation of Cauchy type on an $\operatorname{arc} \Sigma$ in the complex plane.

Step 3: We now investigate the right-hand side of (2.49). We therefore turn back to the parametrized version of (2.49) finding that $h(\gamma(s)) \in C^{0, \alpha}$ due to the sufficient regularity of $\Gamma$ and the double-layer potential.

For the other summand of the right hand-side we will consider the parametrized kernel

$$
k\left(e^{\frac{i \pi t}{L}}, e^{\frac{i \pi \tau}{L}}\right) \frac{i \pi}{L} e^{\frac{i \pi \tau}{L}}=\frac{1}{2 \pi}\left(\frac{\left\langle t_{\Gamma}(\gamma(t)), \gamma(t)-\gamma(\tau)\right\rangle}{|\gamma(t)-\gamma(\tau)|^{2}}+\frac{i \pi}{L} \frac{e^{\frac{i \pi \tau}{L}}}{e^{\frac{i \pi \tau}{L}}-e^{\frac{i \pi t}{L}}}\right)
$$

and show that it can be continuously extended for $t=\tau$ together with its first order derivative with respect to $t$. We first note from (2.50) that for $t \neq \tau$ the kernel is obviously continous and continously differentiable with respect to $t$. For the continuous extension for $t=\tau$ we calculate

$$
\begin{aligned}
2 \pi k\left(e^{\frac{i \pi t}{L}}, e^{\frac{i \pi \tau}{L}}\right) \frac{i \pi}{L} e^{\frac{i \pi \tau}{L}} & \frac{\left\langle t_{\Gamma}(\gamma(t)), \gamma(t)-\gamma(\tau)\right\rangle}{|\gamma(t)-\gamma(\tau)|^{2}}+\frac{i \pi}{L} \frac{e^{\frac{i \pi \tau}{L}}}{e^{\frac{i \pi \tau}{L}}-e^{\frac{i \pi s}{L}}} \\
= & \frac{\left\langle t_{\Gamma}(\gamma(t)), \gamma(t)-\gamma(\tau)\right\rangle}{|\gamma(t)-\gamma(\tau)|^{2}}+\frac{\pi}{2 L} \cot \left(\frac{\pi}{2 L}(\tau-t)\right)+\frac{i \pi}{2 L} \\
= & \frac{\left\langle t_{\Gamma}(\gamma(t)), \gamma(t)-\gamma(\tau)\right\rangle(\tau-t)+|\gamma(t)-\gamma(\tau)|^{2}}{|\gamma(t)-\gamma(\tau)|^{2}(\tau-t)}+\frac{i \pi}{2 L} \\
& \quad+\frac{\pi}{2 L} \cot \left(\frac{\pi}{2 L}(\tau-t)\right)-\frac{1}{\tau-t} .
\end{aligned}
$$

The identity for the cotangens used above is a clever but straightforward computation which can be found for example in [47, 49]. From the series expansion of the cotangens around $\tau-t=0$ we observe that in the series expansion of

$$
\frac{\pi}{2 L} \cot \left(\frac{\pi}{2 L}(\tau-t)\right)-\frac{1}{\tau-t}
$$

around $\tau-t=0$ the smallest term is of order $(\tau-t)^{1}$. Hence (2.51) can be continuously extended for $t=\tau$ together with its first order derivative with respect to $t$. Using the Taylor expansions

$$
\begin{aligned}
& \gamma(t)-\gamma(\tau)=(t-\tau) \int_{0}^{1} \gamma^{\prime}(\tau+\lambda(t-\tau)) d \lambda \\
& \gamma(\tau)-\gamma(t)=\gamma^{\prime}(t)(\tau-t)+(\tau-t)^{2} \int_{0}^{1}(1-\lambda) \gamma^{\prime \prime}(t+\lambda(\tau-t)) d \lambda
\end{aligned}
$$


we find that the other term in the representation of the kernel can be written as

$$
\begin{aligned}
& \frac{\left\langle t_{\Gamma}(\gamma(t)), \gamma(t)-\gamma(\tau)\right\rangle(\tau-t)+|\gamma(t)-\gamma(\tau)|^{2}}{|\gamma(t)-\gamma(\tau)|^{2}(\tau-t)} \\
& =-\frac{\left\langle\gamma(\tau)-\gamma(t)-\gamma^{\prime}(t)(\tau-t), \gamma(t)-\gamma(\tau)\right\rangle}{|\gamma(t)-\gamma(\tau)|^{2}(\tau-t)} \\
& =\frac{\left\langle\int_{0}^{1}(1-\lambda) \gamma^{\prime \prime}(t+\lambda(\tau-t)) d \lambda, \int_{0}^{1} \gamma^{\prime}(\tau+\lambda(t-\tau)) d \lambda\right\rangle}{\left|\int_{0}^{1} \gamma^{\prime}(\tau+\lambda(t-\tau)) d \lambda\right|^{2}} .
\end{aligned}
$$

Since the denominator remains finite for $(t-\tau) \rightarrow 0$ this establishes that $k$ can be continuously extended. Using the quotient rule to differentiate the above expression with respect to $t$, we see that the denominator just gets squared. Hence, also the first order derivative of $k$ with respect to $t$ can be continuously extended for $t=\tau$.

Now we can conclude that the second term of the right-hand side of (2.49) given by

$$
-2 i \int_{0}^{|\Gamma|} k\left(e^{\frac{i \pi t}{L}}, e^{\frac{i \pi \tau}{L}}\right) \frac{i \pi}{L} e^{\frac{i \pi \tau}{L}} \widetilde{\varphi}\left(e^{\frac{i \pi \tau}{L}}\right) d \tau
$$

is continuously differentiable on $[0,|\Gamma|]$, since the integrand is continuously differentiable with respect to $t$ on $[0,|\Gamma|]$, and since the integrand and its derivative with respect to $t$ are integrable with respect to $\tau$ with an integrable majorant given by

$$
M_{1 / 2}\left|\left[\frac{\partial u}{\partial \nu_{\Gamma}}\right](\gamma(\tau))\right|
$$

where

$$
M_{1}:=\sup _{(t, \tau) \in[0,|\Gamma|]^{2}}\left|k\left(e^{\frac{i \pi t}{L}}, e^{\frac{i \pi \tau}{L}}\right) \frac{i \pi}{L} e^{\frac{i \pi \tau}{L}}\right|, \quad M_{2}:=\sup _{(t, \tau) \in[0, \mid \Gamma]^{2}}\left|\widetilde{k}_{t}(t, \tau)\right| .
$$

The Hölder continuity is then obtained by embedding.

Step 4: Summarizing, we have shown in the first three steps that $\left[\frac{\partial u}{\partial \nu_{\Gamma}}\right]$ is a solution to the integral equation (2.48), which can be regarded as a Cauchy type integral equation over a section of the complex unit circle with a Hölder continuous right-hand side. Thus, we can apply Theorem 2.13 and Corollary 2.14, and it follows that the solution

$$
\widetilde{\varphi}(\zeta)=\left[\frac{\partial u}{\partial \nu_{\Gamma}}\right](\gamma(\arg \zeta))
$$

to the Cauchy integral equation (2.49) vanishes at all endpoints of $\Sigma$, where it is bounded and develops singularities of order $-\frac{1}{2}$ at all endpoints, where it is unbounded. 
The result of Theorem 2.15 falls short against the result achieved in [34] by Hayashi. There, the argument is considered from the opposite end. It is proven that a solution to an equation similar to (2.49) satisfies the analogue of the characterization given in Theorem 2.15. In a second step it is then proven that the solution, which is unbounded at both endpoints, exists for all right-hand sides. In a third step this solution is finally identified as the unique solution to the Direct Scattering Problem for the open arc.

We have just mimicked the first of these steps. Since the results of other steps have already been derived in the methodology of Section 2.3, we were not able to follow the path of [34] beyond the above characterization. Following Hayashi further would have meant to provide an alternative existence theory.

In view of the result of Theorem 2.15 we notice that Lemma 1.15 tells that the density $\widetilde{\varphi}$ associated to $\varphi$ either vanishes at $t=0$ or $t=\pi$ with a zero of order $\alpha_{0}>\frac{1}{2}$, or it does not vanish at all. If it does not vanish, the singularity is of square root type as we have already seen in Lemma 1.15.

With this - still rather vague - characterization of the current distribution (2.38) we finish the investigations into the Boundary Value Problem and the properties of its solution and turn to the related Geometric Optimization Problem. We will return to the Boundary Value Problem in Sections 4.1 and 5.1, where we present an approximation scheme for the solution $u$ and related numerical examples. 


\section{Chapter 3}

\section{A Geometric Optimization Problem}

This chapter is devoted to the Geometric Optimization Problem that occurs in the modelling process for the Meissner state of a thin suprerconducting film from the introduction. We will present the problem in mathematical terms, relate it again to the physical model justifying the mathematical formulations with observations from experimental and theoretical physics.

After studying the range and injectivity of the so-called current mapping that relates the geometry to the corresponding current distribution on $\Gamma$, we derive a factorization for the objective functional of the Geometric Optimization Problem that will be at the heart of the further analysis. We then recall and develop a series of important tools from the theory of differentiation in Banach spaces. Based on these results, we study the differentiable dependence of the objective functional of the Geometric Optimization Problem on perturbations of the domain by presenting a sensitivity analysis for the solution to the Boundary Value Problem 2.1 as well as for the objective functional. The chapter will be closed by an existence result for the minimizer of the Geometric Optimization Problem under suitable assumptions on the geometry.

\subsection{Statement of the Problem}

In this section we will present and motivate the Geometric Optimization Problem which forms the core of the second part of this work. In the introduction we have already mentioned that the field of shape optimization, to which the problem at hand belongs, is concerned with optimization problems, that contain the geometry as a parameter or variable. Hence, we will lay the foundations for the understanding of the Geometric Optimization Problem by defining what we would like to understand as an admissible domain. 
Definition 3.1 (Admissible domain). Let $\Gamma \subset \mathbb{R}^{2}$ be an open arc of class $C^{3}$ in the sense of Definition 1.5. Then a domain $D=\bigcup_{i=1}^{n} D_{i} \subset \mathbb{R}^{2}$ will be called admissible, if it satisfies

- $D$ is bounded, and $\bar{D}$ is contained in $\mathbb{R}^{2} \backslash \Gamma$.

- the boundary $\partial D$ is of class $C^{2}$.

- each component $D_{i}$ is simply connected.

- $\bar{D}_{i} \cap \bar{D}_{j}=\emptyset$ for all $i \neq j$.

The family of admissible domains will be parametrized by the parameter $\theta$. The family of admissible boundaries will be denoted by

$$
\mathcal{U}_{a d}:=\left\{\partial D_{\theta}: D_{\theta} \text { is admissible }\right\} .
$$

If we compare the definition of an admissible domain with the geometric assumptions of the Boundary Value Problem 2.1, we notice that Problem 2.1 can be formulated for each geometric setup $\left(\Gamma, D_{\theta}\right)$ with an admissible domain $D_{\theta}$. As we have proven unique solvability of Problem 2.1 (see Theorem 2.10), we can associate to each geometric setup $\left(\Gamma, D_{\theta}\right)$ a corresponding solution $u=u_{\theta}$ if we assume that the other occuring parameters are fixed and given. Furthermore, in Section 2.4 we have identified the current distribution on $\Gamma$ as a characteristic quantity of the solution $u_{\theta}$ to the Boundary Value Problem for the geometric setup $\left(\Gamma, D_{\theta}\right)$ (see Lemma 2.12). This motivates the following definition.

Definition 3.2 (Current mapping). Let $\Gamma \subset \mathbb{R}^{2}$ be an open arc of class $C^{3}$ in the sense of Definition 1.5, and let $I \neq 0, \mu_{e}>0$ and $\mu_{i}>0, i=1, \ldots, n$, be given. Then the mapping

$$
\begin{aligned}
\widetilde{F}: \mathcal{U}_{a d} & \rightarrow C^{*}(\Gamma), \\
\partial D_{\theta} & \mapsto\left[\frac{\partial u_{\theta}}{\partial \nu_{\Gamma}}\right],
\end{aligned}
$$

where $\left[\frac{\partial u_{\theta}}{\partial \nu_{\Gamma}}\right]$ is given by (2.38), is called current mapping. It is well-defined by virtue of Theorem 2.10.

The current mapping associates the shape of the boundary of $D$ to the jump of the normal derivative across the open arc, or, speaking in terms of the application which is behind the Boundary Value Problem, it is linking the form of the magnetic environment to the current distribution in the superconducting film. Based on this correspondence we can now formulate the Geometric Optimization Problem using the language of shape optimization. 
Problem 3.3 (Geometric Optimization Problem). Let $\Gamma \subset \mathbb{R}^{2}$ be an open arc of class $C^{3}$ in the sense of Definition 1.5, and let the parameters $I \neq 0, \mu_{e}>0$ and $\mu_{i}>0, i=1, \ldots, n$, be given. Consider

$$
\min _{\partial D_{\theta} \in \mathcal{U}_{a d}} F\left(\partial D_{\theta}\right)
$$

where

$$
F\left(\partial D_{\theta}\right):=\frac{1}{2} \int_{\Gamma} w(x)\left[\widetilde{F}\left(\partial D_{\theta}\right)-\frac{I}{|\Gamma|}\right]^{2} d s
$$

for the weight function $w: \Gamma \rightarrow \mathbb{R}_{0}^{+}$given by

$$
w(\gamma(\cos t)):=\left|\gamma^{\prime}(\cos t) \sin t\right|, \quad t \in[0, \pi] .
$$

Before analyzing Problem 3.3 in the subsequent sections of this chapter, let us pick up the discussion from the introduction in order to justify the choice of the functional (3.4) and the weight function defined in (3.5). For the moment, let us remark that the weight function $w$, as it is defined in (3.5), depends on the parametrization $\gamma$ of $\Gamma$, although this is not a necessary feature. We will discuss the advantages and drawbacks of the choice (3.5) for $w$ in more detail at the end of this section.

Experiments with superconducting materials (see, for example [41, 43]) as well as the basic theory on superconductivity (see [11, 40]) indicate that the Meissner state of a superconducting medium does not only depend on the temperature, but also (and mainly) on the magnetic field exterior to the medium. In particular, a breakdown of the Meissner state of a superconducting medium may be caused by two different phenomena.

In a first scenario, the magnetic field $\mathbf{B}$ starts to penetrate the superconducting medium at some critical value of first flux entry $H^{*}$. This marks a breakdown of the Meissner state, which is defined through $\mathbf{B}=0$ inside the superconducting medium. Nevertheless, the medium still remains in a superconducting state, the so-called Shubnikov phase, even for field values larger than $H^{*}$. In the Shubnikov phase, the magnetic field already $\mathbf{B}$ penetrates the superconducting medium, but due to geometric pinning forces it is detained from moving, keeping it thus in a superconducting state up to some other critical value $H_{c_{2}}$, above which also the Shubnikov phase breaks down, and the medium becomes normally conducting. It has been shown experimentally (see $[10,41]$ ) that the penetration of the magnetic field into the superconducting medium mainly occurs at defects in the material structure of the medium. Since the edges of a superconducting film naturally form defects in the material structure, this is where the penetration of the film usually starts.

In a second scenario, the magnetic field $\mathbf{B}$ is retained from penetrating the superconducting medium even for field values higher that $H^{*}$ due to some magnetic 
or geometric forces. Even in this case the Meissner state cannot be maintained for arbitrary large field values. The reason for this is a microscopic quantity, the so-called Ginzburgh-Landau depairing current $j_{0}$ (see [8]), which depends on local material properties. The magnetic field $\mathbf{B}$ generates so-called shielding currents in the superconducting medium, which cause a breakdown of the Meissner state to the normally conducting state if the current density distribution exceeds $j_{0}$ locally. In [8] it has also been shown that the shielding currents generated in the Shubnikov phase by field values of $H_{c_{2}}$ are still one order of magnitude smaller than $j_{0}$.

From these two scenarios we can now formulate the objective in terms of the mathematical model. An optimized magnetic environment for the Meissner state of a superconducting film should meet two main issues:

- Firstly, it should aim at reducing the values of the magnetic field locally near the endpoints of the film in order to prevent the Meissner state from collapsing into the Shubnikov phase.

- Secondly it should aim at increasing the total current carrying capability of the film being completely in Meissner state. In view of Ampère's law (5), this corresponds to increasing the local values of the current distribution in the film which are uniformly bounded by the depairing current $j_{0}$.

Interestingly, the mathematical model which we have used for the Boundary Value Problem 2.1 is linear with respect to the transport current $I$. This can be seen immediately from the right-hand side of the linear system (2.13). This is an unphysical behaviour which renders it impossible to directly maximize the total transport current. Even maximization with respect to $I$ under the constraint

$$
\left[\frac{\partial u_{\theta}}{\partial \nu_{\Gamma}}\right]<j_{0}
$$

is not sensible due to the possibly singular behaviour of the current distribution at the endpoints of $\Gamma$ (see Theorem 2.15). Consequently we will, for a fixed total current, optimize the shape of the magnetic environment with the aim of homogenizing the current distribution over the cross-section of the film. In a second step one can then determine the maximal total current under the side constraint that (3.6) holds on $\Gamma$ apart from small neighbourhoods of the endpoints. This is done by exploiting the linear relation between the total current $I$ and the current distribution $\left[\frac{\partial u_{\theta}}{\partial \nu_{\Gamma}}\right]$ that is apparent from (2.40) and (2.13). The exclusion of small neighbourhoods of the endpoints for the side constraint is also justified from the physical perspective. It is well accepted that at the edges of a superconducting medium additional phenomena influence the behaviour of the medium. These phenomena, which are called barrier effects, have not been incorporated into the model making it reliable only away from the edges of the medium. Focussing 
again on the objective of the Geometric Optimization Problem, the discussion has shown that it is the primary goal to homogenize the current distribution. This then leads naturally to the choice of a differentiable $L_{2}$-type functional for the current distribution with the target value $\frac{I}{|\Gamma|}$ being a perfectly homogeneous current distribution for the total current $I$.

The characterization of the current distribution in Theorem 2.15 has stated two possibilities for the behaviour of $\left[\frac{\partial u_{\theta}}{\partial \nu_{\Gamma}}\right]$ at the endpoints, namely either an unbounded behaviour, or the vanishing of the current distribution at the endpoints. These possibilities in view, the question arises why one does not try to optimize in such a way that the second possibility holds, since this would be the best way of dealing with the first of the two main issues stated above.

As an answer we adduce two arguments. Firstly, the overall objective is to maximize the current carrying capability under the restriction (3.6). In view of this, an almost homogeneous current is the best one can do regardless of the behaviour in small neighbourhoods of the endpoints, which are negligible anyway due to the argument given above. Secondly, Theorem 2.15 only gives necessary conditions for the behaviour of the current distribution at the endpoints. In our numerical experience the case of a current distribution vanishing at the endpoints has never occured, and we have not been able to give sufficient conditions for this case to occur either. The choice (3.4) for the objective functional is not prejudiced with respect to the behaviour at the endpoint anyway. It will simply favour the behaviour that allows the largest current transport.

Let us close this section with a short remark on the weight function $w$ occuring in (3.4). The current mapping $\widetilde{F}$ maps into the space $C^{*}(\Gamma)$, which can be seen from (2.40). For the elements of $C^{*}(\Gamma)$ we have shown in Lemma 1.15 that they are square integrable if and only if the related functions $\widetilde{\varphi} \in C_{2 \pi, e}^{0, \alpha}$ vanish at the endpoints of $\Gamma$. In view of the result of Theorem 2.15 we immediately see that the current distribution is not square integrable if the function $f_{ \pm 1}^{*}$ in (2.46) does not vanish at $z_{ \pm 1}$. During our numerical studies of the Geometric Optimization Problem we have experienced no situation where either $f_{ \pm 1}^{*}$ or the related function $\widetilde{\varphi}$ vanished at the endpoints. Hence, a classical $L_{2}$-functional is inacceptible. A closer study of the proof of Lemma 1.15 reveals that the introduction of a weight function $w$, vanishing at the endpoints with a whatsoever small degree, restores the square integrability for elements of $C^{*}(\Gamma)$. The particular choice (3.5) for $w$ then has the critical advantage that it inverts the process of how $\varphi \in C^{*}(\Gamma)$ is obtained from the well-behaved, square-integrable function $\widetilde{\varphi} \in C_{2 \pi, e}^{0, \alpha}$ as can be seen from the definition of $C^{*}(\Gamma)$. We will see in the next section that this choice for $w$ yields a particularly simple and appealing structure for the functional $F$ due to the above advantage. This most desirable effect outweighs by far the drawback that (3.5) depends on the parametrization $\gamma$. Generally speaking, the reasoning above has shown that the only requirement for a weight function $w$ is that it vanishes at the endpoints $z_{ \pm 1}$ of $\Gamma$. This leaves a considerable amount of freedom 
for a possible choice of $w$ and includes also weight functions that do not depend on the parametrization of $\Gamma$. The choice of an appropriate weight function is a crucial issue of of the design of Problem 3.3 as different weight functions may influence the result of the minimization heavily. Our particular choice for $w$ is less influenced from the point of view of appropriate design, but rather from the appealing structure of $F$ that can be obtained in that way.

\subsection{Global properties of the current mapping and the objective functional}

In this section we analyze the Geometric Optimization Problem 3.3 globally, compiling various properties of the current mapping $\widetilde{F}$ from Definition 3.2 and the functional $F$ defined in (3.4). From the rather vague nature of these properties we draw the conclusion that one should rather exploit local properties of $F$ in order to solve Problem 3.3 numerically.

Considering $F$ in detail, we see that it is built as a weighted $L_{2}$-functional for which one expects nice properties such as convexity. On the other hand it is not obvious how to exploit this desirable behaviour, as the range of the current mapping $\widetilde{F}$ determines the domain on which this $L_{2}$-type functional acts. Since the properties of $\widetilde{F}$ have not yet been studied, we turn to this issue with an immediate observation.

Corollary 3.4. Let $\Gamma \subset \mathbb{R}^{2}$ be an open arc of class $C^{3}$ in the sense of Definition 1.5, and let $I \neq 0, \mu_{e}>0$ and $\mu_{i}>0, i=1, \ldots, n$, be given. Then $a$ function $\varphi$ that is in the range of the current mapping $\widetilde{F}$ can be characterized by

i) $\varphi \in C^{*}(\Gamma)$.

ii) In a neighbourhood $U\left(z_{-1}\right)$ of $z_{-1}$ the function $\varphi$ is either bounded and satisfies $\varphi\left(z_{-1}\right)=0$, or it is unbounded and satisfies

$$
\varphi(x)=\left(\left|x-z_{-1}\right|\right)^{-\frac{1}{2}} f_{-1}(x), \quad x \in \Gamma \cap U\left(z_{-1}\right),
$$

for some bounded function $f_{-1} \in C(\Gamma)$.

iii) The analogue holds for a neighbourhood $U\left(z_{+1}\right)$ of $z_{+1}$. Either the function $\varphi$ is bounded there and satisfies $\varphi\left(z_{+1}\right)=0$, or it is unbounded in $U\left(z_{+1}\right)$ and satisfies the analogue of (3.7) for some bounded function $f_{+1} \in C(\Gamma)$.

In particular, the range of $\widetilde{F}$ does not comprise the constants, i.e.

$$
\widetilde{F}\left(\partial D_{\theta}\right) \neq \text { const } \quad \text { for all } \partial D_{\theta} \in \mathcal{U}_{a d}
$$


Proof. The first property is a consequence of Lemma 2.12, the second and third property are immediate from Theorem 2.15. The final statement can be seen as follows. Suppose there is a constant in the range of $\widetilde{F}$. Due to Lemma 2.12 this constant has to be given by $\frac{I}{|\Gamma|}$ as it needs to be current distribution for the parameter $I$. Now $\frac{I}{|\Gamma|}$ is bounded at both endpoints $z_{ \pm 1}$. According to the second and third property, it has to satisfy $\frac{I}{|\Gamma|}=0$, which implies that $I=0$. This is a contradiction to the assumption.

From Corollary 3.4 we see that we cannot decide immediately whether the Geometric Optimization Problem 3.3 is uniquely solvable or not. This is meant in the sense that it is not clear whether there exists a uniquely determined admissible domain $D_{\theta}$ such that the functional $F$ from (3.4) satisfies $F\left(\partial D_{\theta}\right)=0$, as the constants are not in the range of $\widetilde{F}$.

As it seems rather hard to find a stronger characterization for the range of $\widetilde{F}$, we will not pursue this issue any further and turn towards studying the injectivity of $\widetilde{F}$.

Lemma 3.5. Let $\partial D_{\theta_{i}} \in \mathcal{U}_{a d}, i=1,2$, such that

$$
\widetilde{F}\left(\partial D_{\theta_{1}}\right)=\widetilde{F}\left(\partial D_{\theta_{2}}\right) .
$$

Then the first components $\widetilde{\varphi}_{\theta_{i}}$ of the solutions $\Psi_{i}$ to (2.36), corresponding to the solutions $u_{\theta_{i}}$ to Problem 2.1 for the geometry $\left(\Gamma, D_{\theta_{i}}\right)$, differ at most by a multiple of $w$ as given by (3.5).

Proof. Let $u_{\theta_{1}}$ be the solution to Problem 2.1 for the geometry $\left(\Gamma, D_{\theta_{1}}\right)$. Then the current distribution for $u_{\theta_{1}}$ on $\Gamma$ is given by

$$
\widetilde{F}\left(\partial D_{\theta_{1}}\right)=\varphi_{\theta_{1}}-\frac{1}{|\Gamma|} \int_{\Gamma} \varphi_{\theta_{1}} d s+\frac{I}{|\Gamma|}
$$

according to (2.40). As a similar expression holds for $u_{\theta_{2}}$, we obtain

$$
\varphi_{\theta_{1}}-\varphi_{\theta_{2}}=\frac{1}{|\Gamma|} \int_{\Gamma} \varphi_{\theta_{2}} d s-\frac{1}{|\Gamma|} \int_{\Gamma} \varphi_{\theta_{1}} d s
$$

The right-hand side being constant implies that $\varphi_{\theta_{1}}-\varphi_{\theta_{2}}$ is constant. Now the statement follows, because $\varphi_{\theta_{i}}$ and the first component $\widetilde{\varphi}_{\theta_{i}}$ of the solution $\Psi_{i}$ to the corresponding system of integral equations (2.36) are related via

$$
\widetilde{\varphi}_{\theta_{i}}(t)=\varphi_{\theta_{i}}(\gamma(\cos t))\left|\gamma^{\prime}(\cos t) \sin t\right|
$$

for $i=1,2$. 
Lemma 3.6. Let the open arc $\Gamma$ be given by $\left\{x \in \mathbb{R}^{2}: x_{1} \in[-1,1], x_{2}=0\right\}$, i.e. $\Gamma$ is a segment of the $x_{1}$-axis. Furthermore, let $D_{\theta} \subset \mathbb{R}^{2}$ be an admissible domain that is simply connected and contained in the upper half-plane, i.e. we have $\partial D_{\theta} \in \mathcal{U}_{a d}$ and $\partial D_{\theta} \subset\left\{x \in \mathbb{R}^{2}: x_{2}>0\right\}$. Then the domain $D_{\bar{\theta}}$ given by

$$
D_{\bar{\theta}}:=\left\{\bar{x}=\left(x_{1},-x_{2}\right) \in \mathbb{R}^{2}: x=\left(x_{1}, x_{2}\right) \in D_{\theta}\right\}
$$

is also admissible and different from $D_{\theta}$, and both satisfy

$$
\widetilde{F}\left(\partial D_{\theta}\right)=\widetilde{F}\left(\partial D_{\bar{\theta}}\right) .
$$

Proof. The admissibility of $D_{\bar{\theta}}$ is easily checked from Definition 3.1, it being different from $D_{\theta}$ is immediate as it is contained in the lower half-plane by its definition. From Chapter 2 we know that Problem 2.1 is uniquely solvable for the geometry $\left(\Gamma, D_{\theta}\right)$, and that the solution can be represented in the form of (2.10), where the densities $\left(\varphi_{\theta}, \psi_{\theta}\right)$ solve $(2.13)$ for the according geometry.

Now, let $z$ be a parametrization of $\partial D_{\theta}$ with positive orientation. Then a parametrization of $\partial D_{\bar{\theta}}$ is given by $\bar{z}:=\left(z_{1},-z_{2}\right)$ having negative orientation. This has the effect that the exterior normal $\bar{\nu}(\bar{y})$ to $\partial D_{\bar{\theta}}$ at a point $\bar{y} \in \partial D_{\bar{\theta}}$ satisfies

$$
\bar{\nu}(\bar{y})=-\nu(\bar{y}),
$$

where $\nu(\bar{y})$ is the right-hand normal with respect to the parametrization as defined in Definition 1.5. Consequently, the following relations hold for $x, y \in \partial D_{\theta}$ :

$$
\begin{aligned}
|x-y| & =|\bar{x}-\bar{y}|, \\
\langle\nu(y), x-y\rangle & =\langle\bar{\nu}(\bar{y}), \bar{x}-\bar{y}\rangle=-\langle\nu(\bar{y}), \bar{x}-\bar{y}\rangle,
\end{aligned}
$$

where $\bar{x}, \bar{y} \in \partial D_{\bar{\theta}}$ are the points corresponding to $x, y$ on the mirrored boundary. Similarly, we obtain for $x \in \Gamma$ and $y \in \partial D_{\theta}$

$$
\begin{aligned}
|x-y| & =|x-\bar{y}| \\
\langle\nu(y), x-y\rangle & =\langle\bar{\nu}(\bar{y}), x-\bar{y}\rangle=-\langle\nu(\bar{y}), x-\bar{y}\rangle
\end{aligned}
$$

as $x=\bar{x}$ for $x \in \Gamma$.

If we now define $\bar{\psi} \in C\left(\partial D_{\bar{\theta}}\right)$ via $\bar{\psi}(\bar{y}):=-\psi(y)$, we can conlcude from the form of the operators (2.14) - (2.19) and the above that $(\varphi, \bar{\psi})$ solve the system of integral equations (2.13) for the geometry $\left(\Gamma, D_{\bar{\theta}}\right)$ if and only if $(\varphi, \psi)$ solve the corresponding system for the geometry $\left(\Gamma, D_{\theta}\right)$. It follows in particular the operators (2.14) and (2.15) satisfy

$$
\left(K^{D_{\bar{\theta}}} \bar{\psi}\right)(\bar{x})=\left(K^{D_{\theta}} \psi\right)(x), \quad\left(K^{D_{\bar{\theta}}, \Gamma} \bar{\psi}\right)(z)=\left(K^{D_{\theta}, \Gamma} \psi\right)(z)
$$


for all $x \in \partial D_{\theta}$ and for all $z \in \Gamma$, as the '-'-sign in the definition of $\bar{\psi}$ is cancelled by the additional '-'-sign coming from the unit normal in the kernel for the mirrored boundary $\partial D_{\bar{\theta}}$. The operators (2.17) and (2.19) also satisfy

$$
\left(S^{\Gamma, D_{\bar{\theta}}} \varphi\right)(\bar{x})=\left(S^{\Gamma, D_{\theta}} \varphi\right)(x), \quad\left(\widetilde{S}^{\Gamma, D_{\bar{\theta}}} \varphi\right)(\bar{x})=\left(\widetilde{S}^{\Gamma, D_{\theta}} \varphi\right)(x)
$$

as the kernels for $x \in \partial D_{\theta}$ and $\bar{x} \in \partial D_{\bar{\theta}}$ coincide. Finally, the operators (2.16) and (2.18) remain unaffected as they do not depend on $D_{\theta}$. Hence, $\left(\varphi_{\theta}, \bar{\psi}_{\theta}\right)$ is a solution to $(2.13)$ for the geometry $\left(\Gamma, D_{\bar{\theta}}\right)$. Now the result follows from the representation (2.40) for the image of the current mapping $\widetilde{F}$.

Lemma 3.5 and Lemma 3.6 have indicated two additional difficulties, when we want to characterize the current mapping $\widetilde{F}$. Firstly, the densities characterizing a solution $u$ of the Boundary Value Problem 2.1 are not mapped injectively to the corresponding current distribution, but they may differ by some multiple of the weight function $w$ from (3.5) and still lead to the same current distribution. Secondly, we have found an example where two different admissible domains $D_{\theta}$ and $D_{\bar{\theta}}$ lead to the same density on $\Gamma$ when solving the system (2.36). Hence, these two domains also incur the same current distribution.

In view of these difficulties in characterizing both the range and the 'nullspace' of the current mapping we conclude that studying the current mapping itself is probably not the best way of dealing with the Geometric Optimization Problem 3.3. We will therefore turn away from the considerations about a global characterization of the current mapping. Instead we will derive a factorization for the functional $F$ which on the one hand clearly separates the various phenomena that have occured in the above, and which on the other hand turns out to be extremely apt for analyzing a possible differentiable dependence of $\widetilde{F}$ and $F$ on perturbations of the admissible domain $D_{\theta}$.

We begin by deriving a parametrized representation for $F$. We use (2.40) to express the image of the current mapping $\widetilde{F}$ in terms of the density $\varphi_{\theta} \in C^{*}(\Gamma)$ corresponding to the solution $u_{\theta}$ for the geometry $\left(\Gamma, D_{\theta}\right)$. Then we can insert the parametrization $\gamma$ for the arc $\Gamma$ and perform the cosine substitution as in Chapter 2. Finally, the definition of $C^{*}(\Gamma)$ together with the specific form of the weight function $w$ from (3.5) yields

$$
\begin{aligned}
F\left(\partial D_{\theta}\right) & =\frac{1}{2} \int_{\Gamma} w(x)\left[\varphi_{\theta}(x)-\frac{1}{|\Gamma|} \int_{\Gamma} \varphi_{\theta} d s\right]^{2} d s(x) \\
& =\frac{1}{2} \int_{0}^{\pi}\left[\varphi_{\theta}(\gamma(\cos t))-\frac{1}{|\Gamma|} \int_{0}^{\pi} \widetilde{\varphi}_{\theta} d s\right]^{2}\left|\gamma^{\prime}(\cos t) \sin t\right| w(\gamma(\cos t)) d t \\
& =\frac{1}{2} \int_{0}^{\pi}\left[\widetilde{\varphi}_{\theta}(t)-\left(\frac{1}{|\Gamma|} \int_{0}^{\pi} \widetilde{\varphi}_{\theta} d s\right)\left|\gamma^{\prime}(\cos t) \sin t\right|\right]^{2} d t
\end{aligned}
$$


where $\widetilde{\varphi}_{\theta}$ is the first component of the solution $\Psi_{\theta}$ to the system of integral equations (2.36) for the geometry $\left(\Gamma, D_{\theta}\right)$.

With this representation for $F$ in hand we can now factorize $F$ in a way such that each factor either incorporates a desirable property or clearly characterizes one of the various phenomena that have occured in the discussion above. We introduce the operator

$$
\begin{aligned}
T: \mathcal{U}_{a d} & \rightarrow W_{0}=W_{0}\left(\partial D_{\theta}\right) \\
\partial D_{\theta} & \mapsto \Psi_{\theta},
\end{aligned}
$$

where $\Psi_{\theta} \in W_{0}\left(\partial D_{\theta}\right)$ is the solution to $(2.36)$ for the geometry $\left(\Gamma, D_{\theta}\right)$. Next we introduce the projection

$$
\begin{aligned}
P_{\Gamma}: W_{0}\left(\partial D_{\theta}\right) & \rightarrow C_{2 \pi, e}^{0, \alpha} \\
\Psi_{\theta}=\left(\widetilde{\varphi}_{\theta}, \psi_{1, \theta}, \ldots, \psi_{n, \theta}\right) & \mapsto \widetilde{\varphi}_{\theta}
\end{aligned}
$$

as well as the operator $M_{\gamma}: C_{2 \pi, e}^{0, \alpha} \rightarrow C_{2 \pi, e}^{0, \alpha}$ that is defined via

$$
\left(M_{\gamma} \widetilde{\varphi}\right)(t):=\widetilde{\varphi}(t)-\left(\frac{1}{|\Gamma|} \int_{0}^{\pi} \widetilde{\varphi} d s\right)\left|\gamma^{\prime}(\cos t) \sin t\right|
$$

and the functional $G: C_{2 \pi, e}^{0, \alpha} \rightarrow \mathbb{R}$ given by

$$
G \widetilde{\varphi}:=\frac{1}{2} \int_{0}^{\pi}(\widetilde{\varphi}(t))^{2} d t
$$

Using the parametrized representation for $F$ we see that (3.9) - (3.12) yield the factorization

$$
F\left(\partial D_{\theta}\right)=\left(G \circ M_{\gamma} \circ P_{\Gamma} \circ T\right)\left(\partial D_{\theta}\right),
$$

which is more appropriate for the necessary analysis. We state the properties of the functional $G$ and the operator $M_{\gamma}$ in the following two lemmas.

Lemma 3.7. Let $G: C_{2 \pi, e}^{0, \alpha} \rightarrow \mathbb{R}$ be given by (3.12). Then $G$ is a non-negative, convex functional, i.e. it has the property that $\varphi \equiv 0$ is the only local minimizer. Hence $\varphi \equiv 0$ is a global minimizer and satisfies $G(\varphi)=0$.

Proof. First we note that $G(\varphi)$ is obtained by integrating a non-negative integrand, hence $G$ is non-negative. Furthermore, we know from the properties of the integral that $G(\varphi)=0$ if and only if $\varphi \equiv 0$. Hence $\varphi \equiv 0$ is a uniquely determined global minimizer for $G$. 
Hence it remains to show that there exist no other local minimizers for $G$. So let us assume that $\psi \in C_{2 \pi, e}^{0, \alpha}$ is a local minimizer of $G$. For arbitrary $\varphi \in C_{2 \pi, e}^{0, \alpha}$ and for $t \in \mathbb{R}$ with $|t|$ sufficiently small we calculate

$$
G(\psi+t \varphi)=G(\psi)+t \int_{0}^{\pi} \psi \varphi d s+t^{2} G(\varphi)
$$

which yields

$$
t\left(t \cdot G(\varphi)+\int_{0}^{\pi} \psi \varphi d s\right) \geq 0 .
$$

The left hand side of (3.14) is either linear in $t$ or a parabola with a zero at $t=0$. In both cases (3.14) can only be satisfied for $t$ in a neighbourhood of zero if we have

$$
\int_{0}^{\pi} \psi \varphi d s=0
$$

for all $\varphi \in C_{2 \pi, e}^{0, \alpha}$. Inserting $\varphi=\psi$ into (3.15) we conclude that $\psi \equiv 0$.

Lemma 3.8. The operator $M_{\gamma}$ given by (3.11) is linear and bounded from $C_{2 \pi, e}^{0, \alpha}$ to $C_{2 \pi, e}^{0, \alpha}$. It has a non-trivial nullspace which is spanned by $\psi_{0} \in C_{2 \pi, e}^{0, \alpha}$, which is the $2 \pi$-periodic and even extension of $\left|\gamma^{\prime}(\cos t) \sin t\right|$.

Proof. To ensure the mapping properties we have to show that

$$
\left|\gamma^{\prime}(\cos t) \sin t\right| \in C_{2 \pi, e}^{0, \alpha} .
$$

$2 \pi$-periodicity and evenness are satisfied by appropriate extension from $[0, \pi]$ to $[-\pi, \pi]$. For the Hölder regularity with Hölder exponent $\alpha$ we re-write the extension of the above as

$$
|\sin t| \sqrt{\left(\gamma_{1}^{\prime}(\cos t)\right)^{2}+\left(\gamma_{2}^{\prime}(\cos t)\right)^{2}}, \quad t \in \mathbb{R} .
$$

Now $|\sin t|$ is an element of $C^{0,1}$ since the sine is differentiable and the absolute value satisfies the second triangle inequality which is nothing else than the Hölder condition with Hölder exponent 1 and constant $C=1$.

For the second factor in (3.16) we note that the radicand is differentiable and different from zero for all $t \in \mathbb{R}$ since $\gamma$ is a regular parametrization of $\Gamma$. Hence the second factor is continuously differentiable, and consequently (3.16) is in $C_{2 \pi, e}^{0,1}$ and by embedding also in $C_{2 \pi, e}^{0, \alpha}$.

For the nullspace we observe immediately from the definition (3.11) that if $\psi$ in the nullspace of $M_{\gamma}$ and satisfies furthermore $\int_{\Gamma} \psi d s=0$ then $\psi \equiv 0$. If $\psi$ additionally satisfies $\int_{\Gamma} \psi d s \neq 0$ this yields

$$
\left(\frac{1}{|\Gamma|} \int_{\Gamma} \psi d s\right)^{-1} \psi=\left|\gamma^{\prime}(\cos t) \sin t\right|
$$


Hence, $\psi=c\left|\gamma^{\prime}(\cos t) \sin t\right|$ for some constant $c \in \mathbb{R}$, indicating that the nullspace is at most one-dimensional. For the other inclusion we note that

$$
\frac{1}{|\Gamma|} \int_{0}^{\pi}\left|\gamma^{\prime}(\cos t) \sin t\right| d t=\frac{1}{|\Gamma|} \int_{\Gamma} 1 d s=1
$$

Thus $\psi_{0}$, being the $2 \pi$-periodic and even extension of $\left|\gamma^{\prime}(\cos t) \sin t\right| \not \equiv 0$, is in the nullspace of $M_{\gamma}$, which completes the proof.

The results of Lemma 3.7 and Lemma 3.8 are very promising. On the one hand we see that $G$ is most appropriate in order to obtain a globally convergent minimization problem as it has the zero-element as the only local and global minimizer. On the other hand we have identified the source for non-injectivity of the current mapping in the sense of Lemma 3.5, and characterized it as the one-dimensional nullspace of the bounded linear operator $M_{\gamma}$.

It remains to take a closer look at the operators $P_{\Gamma}$ and $T$. From the definition of $P_{\Gamma}$ in (3.10) we immediately see that it is a bounded mapping. But we also recognize that we cannot really speak of linearity of $P$ as the arguments $\Psi_{\theta}$ are defined on different spaces. This is because $W_{0}$ depends on the corresponding boundary $\partial D_{\theta}$. Nevertheless, a closer look at the proof of Lemma 3.6 reveals that in this particular case $P$ is responsible for the non-injectivity of $\widetilde{F}$ in the sense of Lemma 3.6 as we have $P_{\Gamma}\left(\Psi_{\theta}\right)=P_{\Gamma}\left(\Psi_{\bar{\theta}}\right)$ although $\Psi_{\theta} \neq \Psi_{\bar{\theta}}$. Finally, the operator $T$ displays most clearly why the global analysis of the current mapping has proved so difficult and little successful. We observe from (2.36) and (2.28) that $T$ depends nonlinearly on the boundary $\partial D_{\theta}$. Moreover, the boundary $\partial D_{\theta}$ influences (2.36) in all occuring quantities. It is not only the operator $(S+A)^{-1}$ which depends on $\partial D_{\theta}$, but also the right-hand side $f$ and the solution $\Psi$ are defined in terms of $\partial D_{\theta}$.

Having the Geometric Optimization Problem 3.3 identified as a nonlinear optimization problem from the field of shape optimization the central difficulties for analyzing Problem 3.3 become evident. Even in classical optimization theory nonlinear optimization problems are hard to analyze. They mostly extend to characterizing optimal solutions in the spirit of the Karush-Kuhn-Tucker Theorem in combination with an examination of the convexity of both the functional and the admissible set. Unfortunately, all these tools are not available in the present case. First of all, it is difficult to speak of convexity of the set of admissible domains as there is not a naturally underlying linear structure (nevertheless, we will present an approach how to equip $\mathcal{U}_{a d}$ locally with an affine structure in Section 3.4). Due to the lack of the notion of global convexity for $\mathcal{U}_{a d}$, it does not make sense to examine the convexity of the functional $F$ either. Finally, the characterization of optimal solutions in the spirit of the KKT-Theorem turns out to be of rather little use as on the one hand the gradient from the classical KKT-Theorem is replaced by the Fréchet derivative, and on the other hand 
the side constraints that characterize an admissible domain cannot be framed as functional equalities or inequalities.

We will therefore turn away from the attempt of analyzing Problem 3.3 globally. Instead we will examine the local properties of $F$, trying to establish a differentiable dependence of $F$ on perturbations of the boundary $\partial D_{\theta} \in \mathcal{U}_{a d}$. We will give basic results on Fréchet differentiability in a broader setting in the next section, before subsequently doing a sensitivity analysis for Problem 2.1 and Problem 3.3 in particular.

\subsection{Fréchet differentiability}

In this section we compile basic general results on differentiation in Banach spaces as they can be found in $[7,42]$ together with several specialized results that stem from the problem at hand. The main result of this section which establishes Fréchet differentiability for integral operators is an extension of a result that is due to Potthast [58]. We will employ the general results obtained in this section for the sensitivity analysis of the solution to the Boundary Value Problem 2.1 and the functional of the Geometric Optimization Problem 3.3 in the subsequent sections.

We begin with the notion of differentiation in Banach spaces.

Definition 3.9 (Fréchet differentiability). Let $X, Y$ be Banach spaces and $U \subset X$ open. A mapping $F: U \rightarrow Y$ is called Fréchet differentiable in $x_{0} \in U$ if there exists a bounded linear mapping $M=M_{x_{0}} \in L(X, Y)$ such that

$$
\left\|F\left(x_{0}+h\right)-F\left(x_{0}\right)-M_{x_{0}} h\right\|_{Y}=o\left(\|h\|_{X}\right) .
$$

We denote by

$$
F^{\prime}\left[x_{0} ; h\right]:=M_{x_{0}} h
$$

the Fréchet derivative of $F$ at $x_{0}$ in direction $h$.

If $F$ is Fréchet differentiable for every point $x_{0} \in U$, we call the mapping

$$
\begin{aligned}
F^{\prime}: U & \rightarrow L(X, Y) \\
x & \mapsto F^{\prime}[x ; \cdot]
\end{aligned}
$$

the Fréchet derivative of $F$. We say that $F$ is continuously Fréchet differentiable if the mapping $F^{\prime}$ is continuous.

We will now review some of the basic properties and tools of Fréchet differentiation that have in most cases a well-known counterpart in classical real analysis. 
Lemma 3.10. Let $X, Y$ be Banach spaces, $U \subset X$ be open and $F: U \rightarrow Y$ be Fréchet differentiable at $x_{0} \in U$. Then $F^{\prime}\left[x_{0} ; \cdot\right]$ is uniquely determined.

Proof. see [42], p. 103.

Lemma 3.11. Let $X, Y$ be Banach spaces and $A: X \rightarrow Y$ be a bounded linear operator. Then $A$ is Fréchet differentiable for all $x \in X$ and the Fréchet derivative of $A$ at the point $x$ is given by

$$
A^{\prime}[x ; \cdot]=A \text {. }
$$

Proof. see [42], p. 104.

Lemma 3.12. Fréchet differentiable mappings are compatible with linear structures and composition:

1. Let $\lambda \in \mathbb{R}, X, Y$ be Banach spaces, $U \subset X$ be open and $F, G: U \rightarrow Y$ be Fréchet differentiable at $x_{0} \in U$. Then also $F+G$ and $\lambda F$ are Fréchet differentiable at $x_{0}$.

2. Let $X, Y, Z$ be Banach spaces, $U \subset X$ be open and $F: U \rightarrow Y$ be Fréchet differentiable at $x_{0} \in U$. Let $V \subset Y$ be open satisfying $y_{0}:=F\left(x_{0}\right) \in V$ and let $G: V \rightarrow Z$ be Fréchet differentiable at $y_{0}$. Then $G \circ F$ is defined in a neighbourhood of $x_{0}$, it is Fréchet differentiable at $x_{0}$ and the derivative is given by

$$
(G \circ F)^{\prime}\left[x_{0} ; \cdot\right]=G^{\prime}\left[y_{0} ; F^{\prime}\left[x_{0} ; \cdot\right]\right]
$$

Proof. see [42], p. 105.

In order to formulate Taylor's Theorem for real-valued mappings from a Banach space we also need to introduce the notion of higher order derivatives.

Definition 3.13 (Higher order derivatives). Let $X, Y$ be Banach spaces, $U \subset X$ open and $F: U \rightarrow Y$ Fréchet differentiable on $U$ with Fréchet derivative $F^{\prime}$. If $F^{\prime}$ is now again Fréchet differentiable in $x_{0} \in U$ we call the bounded bi-linear mapping

$$
\begin{aligned}
F^{\prime \prime}\left[x_{0} ; \cdot, \cdot\right]: X \times X & \rightarrow Y \\
\left(h_{1}, h_{2}\right) & \mapsto F^{\prime \prime}\left[x_{0} ; h_{1}, h_{2}\right]
\end{aligned}
$$

the second derivative of $F$ at $x_{0}$, and we write $F^{\prime \prime}\left[x_{0} ; h\right]:=F^{\prime \prime}\left[x_{0} ; h, h\right]$. 
We mention here that Definition 3.13 already contains an identification for the second derivative. Formally, $F$ is a mapping from $U \subset X$ into $Y$, and the Fréchet derivative $F^{\prime}[x ; \cdot]$ has been introduced as a linear approximation to $F$ in $x$, i.e. we have $F^{\prime}[x ; \cdot] \in L(X, Y)$. The second Fréchet derivative then is a linear approximation to $F^{\prime}[x ; \cdot]$ in $x$, and thus technically belongs to the space $L(X, L(X, Y))$, which can be identified with $L(X \times X, Y)$. Higher order derivatives are then defined recursively by the above procedure.

Theorem 3.14 (Taylor's Theorem). Let $X$ be a Banach space, $U \subset X$ open, let $x_{0} \in U, h \in X$ and suppose that $\left\{x_{0}+t h: t \in[0,1]\right\} \subset U$. Furthermore let $F: U \rightarrow \mathbb{R}$ be $(k+1)$-times continuously Fréchet differentiable on $U$. Then there exists $\tau \in[0,1]$ such that

$$
F\left(x_{0}+h\right)=F\left(x_{0}\right)+\sum_{j=1}^{k} \frac{1}{j !} F^{(j)}\left[x_{0} ; h\right]+\frac{1}{(k+1) !} F^{(k+1)}\left[x_{0}+\tau h ; h\right] .
$$

Proof. see [42], p. 112.

Remark 3.15. We would like to especially draw the attention to the fact that we only have Taylor's theorem available for real-valued mappings instead of Banach space valued mappings. This is due to the fact that for Banach space valued mappings we only have mean value theorems either as norm estimates or in some weak sense in the case of reflexive Banach spaces. Interestingly, we find that the crucial point for the validity of the mean value theorem for the case of onedimensional real analysis is the ordering of the real numbers or the so-called sup-property.

Lemma 3.16. Let $X$ be a Banach space, $U \subset X$ open and $Y:=Y_{1} \times \ldots \times Y_{n}$ be a product of Banach spaces. Setting

$$
\|y\|:=\max _{i=1, \ldots, n}\left\|y_{i}\right\|_{Y_{i}}, \quad y=\left(y_{1}, \ldots, y_{n}\right) \in Y,
$$

the space $Y$ itself becomes a Banach space. Correspondingly, the space $L(X, Y)$ is a Banach space with norm

$$
\|A\|=\max _{i=1, \ldots, n}\left\|A_{i}\right\|, \quad A=\left(A_{1}, \ldots, A_{n}\right) \in L(X, Y) .
$$

Then the mapping $F=\left(F_{1}, \ldots, F_{n}\right): U \rightarrow Y$ is differentiable at $x_{0} \in U$ if and only if for each $i=1, \ldots, n$ the mapping $F_{i}$ is differentiable at $x_{0}$. We then have

$$
F^{\prime}\left[x_{0} ; \cdot\right]=\left(F_{1}^{\prime}\left[x_{0} ; \cdot\right], \ldots, F_{n}^{\prime}\left[x_{0} ; \cdot\right]\right)^{T} .
$$


Proof. see [42], pp. 104.

Lemma 3.17. Let $X:=X_{1} \times \ldots \times X_{n}$ be the product of Banach spaces, let $U_{i} \subset X_{i}, i=1, \ldots, n$, be open subsets and define $U:=U_{1} \times \ldots \times U_{n}$. Let furthermore $Y$ be another Banach space. Then the mapping

$$
F: U \rightarrow Y
$$

is Fréchet differentiable on $U$ if and only if it is continuously partially Fréchet differentiable with respect to all its variables, i.e. all partial Fréchet derivatives

$$
\frac{\partial F}{\partial z_{i}}\left[z_{1}, \ldots, z_{n} ; \cdot\right]: X_{i} \rightarrow Y
$$

exist in $U$ and depend continuously on $\left(z_{1}, \ldots, z_{n}\right)$. In this case the Fréchet derivative of $F$ at $z=\left(z_{1}, \ldots, z_{n}\right) \in X$ in direction $h=\left(h_{1}, \ldots, h_{n}\right) \in U$ is given by

$$
F^{\prime}\left[z_{1}, \ldots, z_{n} ; h_{1}, \ldots, h_{n}\right]=\sum_{i=1}^{n} \frac{\partial F}{\partial z_{i}}\left[z_{1}, \ldots, z_{n} ; h_{i}\right]
$$

Proof. see [42], pp. 108.

Based on these two classical results we want to establish differentiability for parameter dependent linear operators on product spaces, i.e. for matrix valued functions, from the knowledge of differentiability for all components. We therefore give the following two preparatory Lemmas.

Lemma 3.18. Let $X_{1}, X_{2}, Y$ be Banach spaces and $A: X_{1} \rightarrow Y$ be a bounded linear operator. Moreover, let the operator $P_{1}: X_{1} \times X_{2} \rightarrow X_{1}$ be given by $P_{1}\left(\varphi_{1}, \varphi_{2}\right)=\varphi_{1}$. Then the operator $\widetilde{A}: X_{1} \times X_{2} \rightarrow Y$ defined by $\widetilde{A}:=A \circ P_{1}$ is also linear and bounded, and we additionally have $\|\widetilde{A}\|=\|A\|$.

Proof. Linearity of $\widetilde{A}$ is obvious from its definition and the linearity of $A$ and $P_{1}$. For the boundedness we consider a norm on $X_{1} \times X_{2}$ as in Lemma 3.16 via the maximum norm. Then we see on the one hand that

$$
\left\|\widetilde{A}\left(\varphi_{1}, \varphi_{2}\right)\right\|_{Y}=\left\|A\left(P_{1}\left(\varphi_{1}, \varphi_{2}\right)\right)\right\|_{Y} \leq\|A\| \cdot\left\|\varphi_{1}\right\|_{X_{1}} \leq\|A\| \cdot\left\|\left(\varphi_{1}, \varphi_{2}\right)\right\|_{X_{1} \times X_{2}}
$$


from the boundedness of $A$ and the definition of $P_{1}$ and the maximum norm. Thus $\widetilde{A}$ is bounded with norm $\|\widetilde{A}\| \leq\|A\|$. Since

$$
\begin{aligned}
\left\{\left(\varphi_{1}, \varphi_{2}\right) \in X_{1} \times X_{2}:\right. & \left.\left\|\left(\varphi_{1}, \varphi_{2}\right)\right\|_{X_{1} \times X_{2}}=1\right\} \\
& \supset\left\{\left(\varphi_{1}, 0\right) \in X_{1} \times X_{2}:\left\|\left(\varphi_{1}, 0\right)\right\|_{X_{1} \times X_{2}}=1\right\} \\
& \cong\left\{\varphi_{1} \in X_{1}:\left\|\varphi_{1}\right\|_{X_{1}}=1\right\},
\end{aligned}
$$

we calculate on the other hand

$$
\begin{aligned}
\|\widetilde{A}\| & =\sup _{\left\|\left(\varphi_{1}, \varphi_{2}\right)\right\|_{X_{1} \times X_{2}=1}=1}\left\|\left(A \circ P_{1}\right)\left(\varphi_{1}, \varphi_{2}\right)\right\|_{Y} \\
& \geq \sup _{\left\|\left(\varphi_{1}, 0\right)\right\|_{X_{1} \times X_{2}=1}}\left\|A \varphi_{1}\right\|_{Y}=\sup _{\left\|\varphi_{1}\right\|_{X_{1}}=1}\left\|A \varphi_{1}\right\|_{Y}=\|A\|,
\end{aligned}
$$

which yields the equality of the norms.

Lemma 3.19. Let $X, Y_{1}, \ldots, Y_{n}$ be Banach spaces, define $Y:=Y_{1} \times \ldots \times Y_{n}$ and let $F_{i j}: X \rightarrow L\left(Y_{j}, Y_{i}\right)$ be a Fréchet differentiable mapping. Then the mapping

$$
\begin{aligned}
\widetilde{F}_{i j}: X & \rightarrow L\left(Y, Y_{i}\right) \\
x & \mapsto \widetilde{F}_{i j}(x):=F_{i j}(x) \circ P_{j},
\end{aligned}
$$

where $P_{j}: Y \rightarrow Y_{j}$ is defined by $P_{j}\left(\varphi_{1}, \ldots, \varphi_{n}\right):=\varphi_{j}$, is Fréchet differentiable with Frechet derivative

$$
\widetilde{F}_{i j}^{\prime}[x ; \cdot]=F_{i j}^{\prime}[x ; \cdot] \circ P_{j}
$$

Proof. First we note that

$$
\widetilde{F}_{i j}(x+h)-\widetilde{F}_{i j}(x)-F_{i j}^{\prime}[x ; h] \circ P_{j}=\left(F_{i j}(x+h)-F_{i j}(x)-F_{i j}^{\prime}[x ; h]\right) \circ P_{j} .
$$

Hence, by Lemma 3.18 and differentiability of $F_{i j}$ we have

$$
\begin{aligned}
\| \widetilde{F}_{i j}(x+h)- & \widetilde{F}_{i j}(x)-F_{i j}^{\prime}[x ; h] \circ P_{j} \|_{L\left(Y, Y_{1}\right)} \\
& =\left\|F_{i j}(x+h)-F_{i j}(x)-F_{i j}^{\prime}[x ; h]\right\|_{L\left(Y_{j}, Y_{i}\right)}=o(\|h\|),
\end{aligned}
$$

which proves differentiability of $\widetilde{F}_{i j}$ together with the form of the Fréchet derivative.

Now the desired differentiability result for matrix valued functions can be stated as a simple Corollary. 
Corollary 3.20. Let $X, Y_{1}, \ldots, Y_{n}$ be Banach spaces, define $Y:=Y_{1} \times \ldots \times Y_{n}$ and for $i, j=1, \ldots, n$ consider the following mappings:

$$
\begin{aligned}
& A_{i i}: X \quad \rightarrow \quad L\left(Y_{i}\right) \\
& x \mapsto A_{i i}(x): Y_{i} \rightarrow Y_{i} \\
& \psi \mapsto A_{i i}(x) \psi, \\
& A_{i j}: X \quad \rightarrow \quad L\left(Y_{j}, Y_{i}\right) \\
& x \mapsto A_{i j}(x) \quad: \quad Y_{j} \rightarrow Y_{i} \\
& \psi \mapsto A_{i j}(x) \psi .
\end{aligned}
$$

Let $P_{j}: Y \rightarrow Y_{j}$ be defined as in Lemma 3.19. Then the mapping

$$
\begin{aligned}
A: X & \rightarrow L(Y) \\
x & \mapsto A(x):=\left(\sum_{j=1}^{n}\left(A_{i j}(x) \circ P_{j}\right)\right)_{i=1, \ldots, n}
\end{aligned}
$$

is Fréchet differentiable if all its components $A_{i j}$ for $i, j=1, \ldots, n$ are Fréchet differentiable. The Fréchet derivative of $A$ is then given by

$$
\left(\sum_{j=1}^{n} A_{i j}^{\prime}[x ; \cdot] \circ P_{j}\right)_{i=1, \ldots, n}
$$

Proof. The statement follows from using Lemma 3.19 on each $A_{i j}$, building sums for all fixed $i \in\{1, \ldots, n\}$ and using Lemma 3.16 on the sums.

The problem at hand requires also differentiation of operator expressions where the operator itself as well as the argument depend on the variable with respect to which the differentiation is performed. At the first glance one might think of this situation as a simple consequence of the chain rule or product rule, but a closer look reveals that it is neither of the two. We settle this issue in the following Lemma.

Lemma 3.21. Let $X, Y$ be Banach spaces and $U \subset X$ an open subset. Let $F: U \rightarrow L(Y)$ and $f: U \rightarrow Y$ be Fréchet differentiable mappings. Then also the mapping $x \mapsto F(x) f(x) \in Y$ is Fréchet differentiable for all $x \in U$ and its Fréchet derivative at $x_{0} \in U$ in direction $h$ is given by

$$
F^{\prime}[x ; h] f(x)+F(x) f^{\prime}[x ; h] .
$$


Proof. For $x_{0} \in U$ and small $h \in X$ we expand the expression

$$
\begin{aligned}
F\left(x_{0}+h\right) f\left(x_{0}+h\right)-F\left(x_{0}\right) f\left(x_{0}\right)-F^{\prime}\left[x_{0} ; h\right] f\left(x_{0}\right)-F\left(x_{0}\right) f^{\prime}\left[x_{0} ; h\right] \\
=F\left(x_{0}+h\right) f\left(x_{0}+h\right)-F\left(x_{0}+h\right) f\left(x_{0}\right)-F\left(x_{0}+h\right) f^{\prime}\left[x_{0} ; h\right] \\
\quad+F\left(x_{0}+h\right) f\left(x_{0}\right)-F\left(x_{0}\right) f\left(x_{0}\right)-F^{\prime}\left[x_{0} ; h\right] f\left(x_{0}\right) \\
\quad+F\left(x_{0}+h\right) f^{\prime}\left[x_{0} ; h\right]-F\left(x_{0}\right) f^{\prime}\left[x_{0} ; h\right] .
\end{aligned}
$$

Now estimating the first line on the right-hand side we find that it is $o(\|h\|)$ due to the Fréchet differentiability of $f$ and since

$$
\left\|F\left(x_{0}+h\right)\right\| \leq\left\|F\left(x_{0}+h\right)-F\left(x_{0}\right)\right\|+\left\|F\left(x_{0}\right)\right\| \leq C_{1}
$$

holds uniformly for all sufficiently small $h \in X$ as a consequence of the continuity of $F$. Similarly we find the second line to be $o(\|h\|)$ due to the Fréchet differentiability of $F$. Continuity of $F$ also yields $o(\|h\|)$ for the third line since it can be estimated by

$$
\left\|F\left(x_{0}+h\right)-F\left(x_{0}\right)\right\| \cdot\left\|f^{\prime}\left[x_{0} ; \cdot\right]\right\| \cdot\|h\| .
$$

Putting the results for all summands together using the triangle inequality the statement of the lemma follows.

Furthermore, the operator which needs to be differentiated may be expressed as the inverse of another operator that is more easily accessible. Hence we include the following result (which also appears in $[58,18]$ ) that expresses the differentiability of the inverse of an invertible operator with respect to some parameter together with the form of its derivative in terms of the differentiability and the form of the derivative of the operator itself.

Theorem 3.22. Let $X$ be a Banach space and $Y$ be a Banach algebra. Assume that $F: U \subset X \rightarrow Y$ is Fréchet differentiable in $x_{0} \in U$ and that there exists a neighbourhood $W$ of $x_{0}$ such that $F(x)$ is invertible for all $x \in W$ and the mapping $F^{-1}: x \mapsto(F(x))^{-1}$ is continuous at $x_{0}$. Then $F^{-1}$ is Fréchet differentiable at $x_{0}$ with Fréchet derivative

$$
\left(F^{-1}\right)^{\prime}\left[x_{0} ; h\right]=-F^{-1}\left(x_{0}\right) F^{\prime}\left[x_{0} ; h\right] F^{-1}\left(x_{0}\right) .
$$


Proof. We rewrite the expression

$$
\begin{aligned}
F^{-1}\left(x_{0}+h\right)- & F^{-1}\left(x_{0}\right)+F^{-1}\left(x_{0}\right) F^{\prime}\left[x_{0} ; h\right] F^{-1}\left(x_{0}\right) \\
= & F^{-1}\left(x_{0}\right) F\left(x_{0}\right) F^{-1}\left(x_{0}+h\right) \\
& \quad-F^{-1}\left(x_{0}\right) F\left(x_{0}+h\right) F^{-1}\left(x_{0}+h\right) \\
& \quad+F^{-1}\left(x_{0}\right) F^{\prime}\left[x_{0} ; h\right]\left(F^{-1}\left(x_{0}\right)-F^{-1}\left(x_{0}+h\right)+F^{-1}\left(x_{0}+h\right)\right) \\
= & F^{-1}\left(x_{0}\right)\left(-F\left(x_{0}+h\right)+F\left(x_{0}\right)+F^{\prime}\left[x_{0} ; h\right]\right) F^{-1}\left(x_{0}+h\right) \\
& +F^{-1}\left(x_{0}\right) F^{\prime}\left[x_{0} ; h\right]\left(F^{-1}\left(x_{0}\right)-F^{-1}\left(x_{0}+h\right)\right) .
\end{aligned}
$$

Continuity of $F^{-1}$ now yields

$$
\left\|F^{-1}\left(x_{0}+h\right)\right\| \leq\left\|F^{-1}\left(x_{0}+h\right)-F^{-1}\left(x_{0}\right)\right\|+\left\|F^{-1}\left(x_{0}\right)\right\| \leq M_{1}
$$

uniformly for sufficiently small $h$. Hence the first term can be estimated by

$$
M_{1} \cdot\left\|F^{-1}\left(x_{0}\right)\right\| \cdot\left\|-F\left(x_{0}+h\right)+F\left(x_{0}\right)+F^{\prime}\left[x_{0} ; h\right]\right\|=o(\|h\|)
$$

due to the differentiability of $F$. Similarly the second term can be estimated by

$$
\left\|F^{-1}\left(x_{0}\right)-F^{-1}\left(x_{0}+h\right)\right\| \cdot\left\|F^{\prime}\left[x_{0} ; \cdot\right]\right\| \cdot\|h\|=o(\|h\|)
$$

uniformly for $h \rightarrow 0$. Now combining (3.27), (3.28) and (3.29) using the triangle inequality, we get

$$
\left\|F^{-1}\left(x_{0}+h\right)-F^{-1}\left(x_{0}\right)+F^{-1}\left(x_{0}\right) F^{\prime}\left[x_{0} ; h\right] F^{-1}\left(x_{0}\right)\right\|=o(\|h\|)
$$

uniformly for $h \rightarrow 0$, which proves differentiability for $F^{-1}$ together with the claimed form for the derivative.

Remark 3.23. From the case of matrices on $\mathbb{R}^{n}$ (see [23]) we know a more intuitive approach to establish the result of Theorem 3.22 by formally differentiating the equation

$$
F\left(x_{0}\right) F^{-1}\left(x_{0}\right)=I d
$$

using the product rule. This approach requires knowledge of the differentiability of $F^{-1}$ which in the case of matrices with real-valued entries can be established independently using Cramer's Rule. We stress the point that this approach is not applicable in the case at hand since we do not assume differentiability of the inverse in the theorem above. 
We close this section on Fréchet differentiability with an adaption of the main result of Potthast [58] to the two-dimensional case. This result establishes Fréchet differentiability for boundary integral operators under suitable assumptions on the kernel.

Theorem 3.24. Let $G_{1}, G_{2} \subset \mathbb{R}^{2}$, define $G_{\Delta}:=\left\{(x, y) \in G_{1} \times G_{2}: x \neq y\right\}$ and let $\mu$ be a measure on $G_{2}$. Furthermore, let $X$ be a Banach space, $U \subset X$ open and convex, and let $f: G_{\Delta} \times U \rightarrow \mathbb{R}$ be a continuous function with the following properties:

i) For all $(x, y) \in G_{\Delta}$ the function $f(x, y, \cdot): U \rightarrow \mathbb{R}$ is twice continuously Fréchet differentiable on $U$.

ii) For all $x \in G_{1}, r \in U$ and $h \in X$ the function $f(x, \cdot, r): G_{2} \rightarrow \mathbb{R}$ and the function $f^{\prime}[x, \cdot, r ; h]: G_{2} \rightarrow \mathbb{R}$ are (improperly) integrable.

iii) For all $r \in U$ and for all $h \in X$ the mapping $A(r)$ defined by

$$
(A(r) \psi)(x):=\int_{G_{2}} f(x, y, r) \psi(y) d \mu(y), \quad x \in G_{1}, \psi \in C\left(G_{2}\right),
$$

and the mapping $\widetilde{A}(r, h)$ defined by

$$
(\widetilde{A}(r, h) \psi)(x):=\int_{G_{2}} f^{\prime}[x, y, r ; h] \psi(y) d \mu(y)
$$

for $x \in G_{1}$ and $\psi \in C\left(G_{2}\right)$ are elements of $L\left(C\left(G_{2}\right), C\left(G_{1}\right)\right)$.

iv) There exists an integrable function $g: G_{\Delta} \rightarrow \mathbb{R}$ such that the integrals

$$
\int_{G_{2}} g(x, y) d \mu(y), \quad x \in G_{1}
$$

are uniformly bounded and such that for all $(x, y) \in G_{\Delta}$ the estimate

$$
\left|f^{\prime \prime}\left[x, y, r ; h_{1}, h_{2}\right]\right| \leq g(x, y)
$$

holds uniformly for all $r \in U$ and for all $h_{1}, h_{2} \in X$ with $\left\|h_{i}\right\| \leq 1, i=1,2$.

Then the mapping $r \mapsto A(r)$ as a mapping from $U$ into $L\left(C\left(G_{2}\right), C\left(G_{1}\right)\right)$ is Fréchet differentiable on $U$ and the Fréchet derivative $A^{\prime}[r ; \cdot]$ is given by (3.31). Furthermore, the Fréchet derivative $A^{\prime}[r ; \cdot]$ is continuous with respect to $r \in U$.

The formulation above deviates from the original result of Potthast in two respects. On the one hand we work on subsets of $\mathbb{R}^{2}$ instead of $\mathbb{R}^{3}$, which turns out to be just a minor change from the original as it does not affect the argumentation within the proof. On the other hand the formulation extends the result of 
Potthast as it also establishes the continuous dependence of the Fréchet derivative $A^{\prime}[r ; \cdot]$ with respect to $r \in U$. As expected, the required assumptions (3.32) are stronger than in [58], where the boundedness of the second derivative of $f$ just needed to be assumed for $h_{1}=h_{2}$. The additional continuity property of the Fréchet derivative will form the key to make Corollary 3.20 work in combination with Theorem 3.24.

Proof of Theorem 3.24. Fix $(x, y) \in G_{\Delta}$ and let $r_{0} \in U$. From openness and convexity of $U$ it follows that $r_{0}+t h \in U$ for all $t \in[0,1]$ and any $h \in X$ with sufficiently small norm. Since $f$ is real-valued we can apply Taylor's theorem (Theorem 3.14) to find

$$
f\left(x, y, r_{0}+h\right)=f\left(x, y, r_{0}\right)+f^{\prime}\left[x, y, r_{0} ; h\right]+f^{\prime \prime}\left[x, y, r_{0}+\tau h ; h, h\right]
$$

for some $\tau \in[0,1]$. From the fact that the estimate (3.32) holds uniformly with respect to $r$ and $h_{i}$ for $\left\|h_{i}\right\| \leq 1,(i=1,2)$ we derive the estimate

$$
\left\|f^{\prime \prime}[x, y, r ; \cdot, \cdot]\right\|=\sup _{\substack{\left\|h_{1}\right\| \leq 1 \\\left\|h_{2}\right\| \leq 1}}\left|f^{\prime \prime}\left[x, y, r ; h_{1}, h_{2}\right]\right| \leq g(x, y)
$$

uniformly with respect to $r$. Consequently the point-wise estimate

$$
\begin{aligned}
\left|f^{\prime \prime}\left[x, y, r_{0}+\tau h_{1} ; h_{2}, h_{1}\right]\right| & \leq\left\|f^{\prime \prime}\left[x, y, r_{0}+\tau h_{1} ; \cdot, \cdot\right]\right\|\left\|h_{1}\right\|\left\|h_{2}\right\| \\
& \leq \sup _{r \in U}\left\|f^{\prime \prime}[x, y, r ; \cdot, \cdot]\right\|\left\|h_{1}\right\|\left\|h_{2}\right\| \\
& \leq g(x, y)\left\|h_{1}\right\|\left\|h_{2}\right\|
\end{aligned}
$$

holds. In particular, for $h=h_{1}=h_{2}$ the estimate reads

$$
\left|f^{\prime \prime}\left[x, y, r_{0}+\tau h ; h, h\right]\right| \leq g(x, y)\|h\|^{2} .
$$

Now using (3.34) we calculate

$$
\begin{aligned}
&\left|\left(A\left(r_{0}+h\right) \psi\right)(x)-\left(A\left(r_{0}\right) \psi\right)(x)-\left(\widetilde{A}\left(r_{0}, h\right) \psi\right)(x)\right| \\
&=\mid \int_{G_{2}} f\left(x, y, r_{0}+h\right) \psi(y) d \mu(y)-\int_{G_{2}} f\left(x, y, r_{0}\right) \psi(y) d \mu(y) \\
& \quad-\int_{G_{2}} f^{\prime}\left[x, y, r_{0} ; h\right] \psi(y) d \mu(y) \mid \\
&=\left|\int_{G_{2}} f^{\prime \prime}\left[x, y, r_{0}+\tau h ; h, h\right] \psi(y) d \mu(y)\right| \\
& \leq\|\psi\|_{\infty} \int_{G_{2}}\left|f^{\prime \prime}\left[x, y, r_{0}+\tau h ; h, h\right]\right| d \mu(y) \\
& \leq\|\psi\|_{\infty}\|h\|^{2} \int_{G_{2}} g(x, y) d \mu(y) .
\end{aligned}
$$


Since the operators on the left-hand side are all elements of $L\left(C\left(G_{2}\right), C\left(G_{1}\right)\right)$ and the integrals on the right-hand side are uniformly bounded with respect to $x \in G_{1}$ we can estimate the above in the operator norm on $L\left(C\left(G_{2}\right), C\left(G_{1}\right)\right)$ by taking the supremum both with respect to $x \in G_{1}$ and $\psi \in C\left(G_{2}\right)$, where $\|\psi\|_{\infty}=1$ to find

$$
\left\|A\left(r_{0}+h\right)-A\left(r_{0}\right)-\widetilde{A}\left(r_{0}, h\right)\right\| \leq C\|h\|^{2} .
$$

This establishes Fréchet differentiability for the mapping $r \mapsto A(r)$ as a mapping from $U$ to $L\left(C\left(G_{2}\right), C\left(G_{1}\right)\right)$, and we have furthermore identified (3.31) as the Fréchet derivative.

For the continuity of $A^{\prime}[r, \cdot]$ we consider $r \in U$ and $\left(r_{n}\right)_{n \in \mathbb{N}} \subset U$ such that $\left\|r_{n}-r\right\|_{X} \rightarrow 0$ as $n \rightarrow \infty$. Setting $h_{n}:=r_{n}-r, n \in \mathbb{N}$, we can apply Taylor's Theorem (Theorem 3.14) for arbitrary $h_{0} \in X$ with $\left\|h_{0}\right\| \leq 1$ to find

$$
f^{\prime}\left[x, y, r_{n} ; h_{0}\right]=f^{\prime}\left[x, y, r ; h_{0}\right]+f^{\prime \prime}\left[x, y, r+\tau h_{n} ; h_{0}, h_{n}\right]
$$

for some $\tau \in[0,1]$. Using (3.33), we can estimate for arbitrary $\varphi \in C\left(G_{2}\right)$ and arbitrary $x \in G_{1}$

$$
\begin{aligned}
\left|\left(A^{\prime}\left[r_{n}, h_{0}\right] \varphi\right)(x)-\left(A^{\prime}\left[r, h_{0}\right] \varphi\right)(x)\right| \\
\leq\|\varphi\|_{\infty} \int_{G_{2}}\left|f^{\prime}\left[x, y, r_{n} ; h_{0}\right]-f^{\prime}\left[x, y, r ; h_{0}\right]\right| d s \\
\quad=\|\varphi\|_{\infty} \int_{G_{2}}\left|f^{\prime \prime}\left[x, y, r+\tau h_{n} ; h_{0}, h_{n}\right]\right| d s \\
\leq\|\varphi\|_{\infty}\left\|h_{0}\right\|\left\|h_{n}\right\| \int_{G_{2}} g(x, y) d s \\
\leq C\|\varphi\|_{\infty}\left\|h_{0}\right\|\left\|r_{n}-r\right\| .
\end{aligned}
$$

Now it follows that

$$
\begin{aligned}
\left\|A^{\prime}\left[r_{n}, \cdot\right]-A^{\prime}[r, \cdot]\right\| & =\sup _{\left\|h_{0}\right\| \leq 1}\left\|A^{\prime}\left[r_{n}, h_{0}\right]-A^{\prime}\left[r, h_{0}\right]\right\| \\
& =\sup _{\left\|h_{0}\right\| \leq 1}\left(\sup _{\|\varphi\| \leq 1}\left\|A^{\prime}\left[r_{n}, h_{0}\right] \varphi-A^{\prime}\left[r, h_{0}\right] \varphi\right\|_{\infty, G_{1}}\right) \\
& \leq \widetilde{C}\left\|r_{n}-r\right\|_{X},
\end{aligned}
$$

i.e. we have continuity for $A^{\prime}[r, \cdot]$ with respect to $r$.

Remark 3.25. We have adapted the generality of Potthast [58] as to the sets $G_{1}$ and $G_{2}$. This allows us to employ Theorem 3.24 for line integrals as well as for volume integrals and it is regardless with respect to the image domain, i.e. it covers continuous and weakly singular boundary integrals and also potential operators. 


\subsection{Domain sensitivity results for the solution to the Boundary Value Problem}

In this section we will consider the sensitivity of the solution $u=u_{\theta}$ to the Boundary Value Problem 2.1 for the geometry $\left(\Gamma, D_{\theta}\right)$ with respect to the shape parameter $\theta$.

In Section 3.2 we have pointed out that the dependence of $u$ and $F$ on the geometry is nonlinear and non-convex. For this class of optimization problems, the general existence and uniqueness results from classical optimization theory, which basically require only continuous dependence of the functional on the shape parameter, are more or less non-constructive and rather impracticable for numerical approximations.

Generally speaking, a numerical solution to an optimization problem is obtained by successive approximations. Starting from an initial guess, we seek to improve the approximand in nonlinear problems by using information that are extracted from the linearization of the functional and the PDE-solution with respect to the shape parameter. However, this procedure requires differentiation with respect to the shape parameter, a field in shape optimization or applied mathematics generally that has been coined sensitivity analysis or shape sensitivity analysis. Work in this area has started in the late seventies and early eighties being mainly inspired from structural mechanics. We refer to Haslinger and Mäkinen [33] for an instructive introduction to the field, and to Sokolowski and Zolesio [70] for a comprehensive study of shape sensitivity that in their case is motivated from a geometric point of view.

Sensitivity analysis has also entered the inverse scattering community with the work of Kirsch [44]. In his approach the so-called shape derivative or domain derivative is characterized by an additional boundary value problem that is either derived directly or via variational methods. In this approach the shape derivative is not obtained completely and explicitly. It is rather a black-box approach that allows to calculate the shape derivative in the direction of a certain given boundary perturbation. In a numerical realization of the approach, one will therefore choose finitely many basis functions for the boundary perturbations, and obtain an approximation to the shape derivative by interpreting the calculated directional derivatives as the projection onto the subspace spanned by these basis functions. Among others, Kirsch [44] and Hettlich [37, 38] have applied this approach successfully.

We also mention here a related approach by Roger [60] employing so-called adjoint solutions. This approach has been picked up recently by Bochniak and Cakoni [9], who established the sensitivity analysis for mixed boundary value problems to the Helmholtz equation. The method of adjoint solutions allows to obtain the shape derivative directly rather than in terms of projections. The key idea here is to start out from the characterization of the shape derivative as the solution 
to an additional boundary value problem, and represent the shape derivative in terms of its Cauchy data on the boundary using Green's Representation Theorem. Now the adjoint solution is defined as the solution to an auxiliary boundary value problem such that one can eliminate the term in Green's Representation formula which depends on the normal derivative of the shape derivative.

Although this method is conceptually appealing, it is only of little practical use. The reason for this is that it turns out that - roughly speaking - one has to solve one adjoint problem for each point where one wants to evaluate the shape derivative.

In the work at hand we will follow a third approach from the mid-nineties which is due Potthast [58]. The approach exploits explicitly the existence of a fundamental solution in, for example, scattering theory or potential theory. The central idea is to perform the shape differentiation directly to the solution of the underlying $\mathrm{PDE}$, which can be represented in terms of layer potentials over the varying boundaries.

Subsequently, we will adapt the results of Potthast, that have been presented in [58] for the three-dimensional Helmholtz equation, to the Laplace equation in two dimensions. Essentially, we will see that the boundary integral formulation of the Problem 2.1 renders an elegant way to prove sensitivity. The main point is to re-write the solution to Problem 2.1 as the composition of Fréchet differentiable mappings where the dependence on the domain enters explicitly as a shape parameter with respect to which these mappings are also differentiable.

So, let us first of all introduce a concept how we can equip the set of admissible domains locally with an affine structure.

Definition 3.26. Let $D_{0}$ be an admissible domain for a given arc $\Gamma$, a given transport current $I$ and given magnetic permeabilities $\mu_{e}, \mu_{1}, \ldots, \mu_{n}$. For some sufficiently small constant $m>0$ consider the space

$$
U^{m, \eta}\left(D_{0}\right):=\left\{\theta \in C_{0}^{2}\left(\mathbb{R}^{2}\right):\|\theta\|_{C^{2}}<m, \operatorname{supp}(\theta) \subset V_{\eta}\left(\partial D_{0}\right)\right\},
$$

where $C_{0}^{2}\left(\mathbb{R}^{2}\right)$ denotes the space of all compactly supported, twice continuously differentiable vector fields on $\mathbb{R}^{2}$ and $V_{\eta}$ is some neighbourhood of $\partial D_{0}$ such that

$$
\operatorname{dist}\left(x, \partial D_{0}\right)<\eta \quad \text { for all } x \in V_{\eta} \text {. }
$$

Then a $C^{2}$-diffeomorphism $I_{\theta}: \mathbb{R}^{2} \rightarrow \mathbb{R}^{2}$ is called a perturbation for $D_{0}$ if it can be represented in the form $I_{\theta}=I d+\theta$ for some $\theta \in U^{m, \eta}\left(D_{0}\right)$. Accordingly, we call a domain $D_{\theta}$ a perturbation of $D_{0}$ if there exists a corresponding perturbation $I_{\theta}$ for $D_{0}$ such that $D_{\theta}=I_{\theta}\left(D_{0}\right)$. The corresponding element $\theta \in U^{m, \eta}\left(D_{0}\right)$ will be called shape parameter. 
This notion of perturbations of a domain is presented along the lines of Potthast [58] and Simon [69]. We compile the properties of perturbations for $D_{0}$ in the following lemma.

Lemma 3.27. Let $D_{0}$ be an admissible domain, and let $U^{m, \eta}\left(D_{0}\right)$ be given as in Definition 3.26. Then the following properties hold:

1. $U^{m, \eta}\left(D_{0}\right)$ is open and convex.

2. The perturbations $D_{\theta}$ of $D_{0}$ are admissible domains for any $\theta \in U^{m, \eta}\left(D_{0}\right)$ provided $\operatorname{dist}\left(\partial D_{0}, \Gamma\right)>\eta$.

3. The mapping $\theta \mapsto u_{\theta}$ that maps $\theta \in U^{m, \eta}\left(D_{0}\right)$ to the corresponding solution to Problem 2.1 for the geometry $\left(\Gamma, D_{\theta}\right)$ is well-defined.

Proof. The first statement follows immediately from the definition of $U^{m, \eta}\left(D_{0}\right)$.

For the second statement we observe that we have $I_{\theta}=\mathrm{Id}$ on $\mathbb{R}^{2} \backslash V_{\eta}\left(\partial D_{0}\right)$. Hence we have for $\operatorname{dist}\left(\partial D_{0}, \Gamma\right)>\eta$ also $D_{\theta} \cap \Gamma=\emptyset$. The regularity of $\partial D_{\theta}$ follows from $I_{\theta}$ being a $C^{2}$-diffeomorphism, the boundedness of $D_{\theta}$ is a consequence of the boundedness of $\theta$. Finally the bound $m$ on the $C^{2}$-norm of $\theta$ being sufficiently small ensures that $D_{\theta}$ has the same number of connected components, which are all simply connected.

The last statement follows directly from the second statement and the properties of the admissible domains in connection with the geometric assumptions of Problem 2.1.

With this affine structure for the geometry in hand we can now introduce the occuring mappings for the solution $u=u_{\theta}$ to the Boundary Value Problem 2.1 for the geometry $\left(\Gamma, D_{\theta}\right)$ and formalize the dependence on the domain.

So, let $D_{0}$ be an admissible domain, regard it as a fixed reference domain in the sense of Definition 3.26, and define $U=U^{m, \eta}\left(D_{0}\right)$ accordingly. Then we can according to Lemma 3.27 establish on the one hand a functional dependency between the solution $u_{\theta}$ to Problem 2.1 for the geometry $\left(\Gamma, D_{\theta}\right)$ and the shape parameter $\theta \in U$. On the other hand we know from (2.10) and Theorem 2.10 that we can represent $u_{\theta}$ by applying layer potential operators to the solution of the system of integral equations (2.36). A brief inspection of (2.10) and (2.36) shows that all occuring quantities depend explicitly on the shape parameter $\theta$. In other words, we can re-write the solution $u_{\theta}$ to the Problem 2.1 for an admissible geometry $\left(\Gamma, D_{\theta}\right)$ in the sense of Definition 3.26 in the form

$$
u_{\theta}=P\left(\theta,(S+A)^{-1}(\theta) f(\theta)\right),
$$


denoting explicitly the dependence on the geometry via the shape parameter. Here, the right-hand side $f(\theta)$ to the system of integral equations (2.36) is regarded as a mapping

$$
f: U \rightarrow C_{2 \pi, e}^{1, \alpha} \times C\left(\partial D_{0}\right),
$$

and the solution operator $(S+A)^{-1}(\theta)$ from (2.36) is viewed as a mapping

$$
(S+A)^{-1}: U \rightarrow L\left(C_{2 \pi, e}^{1, \alpha} \times C\left(\partial D_{0}\right), C_{2 \pi, e}^{0, \alpha} \times C\left(\partial D_{0}\right)\right) .
$$

The potential operator $P(\theta, \cdot)$, which is the sum of the double-layer potential operator on the boundary $\partial D_{\theta}$ and modified the single-layer potential operator on the arc $\Gamma$ as given in (2.10), is understood as a mapping

$$
P: U \rightarrow L\left(C_{2 \pi, e}^{0, \alpha} \times C\left(\partial D_{0}\right), \mathcal{A}(\Omega)\right),
$$

where $\Omega \subset \mathbb{R}^{2} \backslash\left(\Gamma \cup \partial D_{0}\right)$ is a compact set depending only on $U$. We emphasize that the mappings are not considered in exactly the same way as in Chapter 2. While there, for example, the operator $(S+A)$ was considered as a mapping from $W_{0}\left(D_{\theta}\right)$ into $W_{1}\left(D_{\theta}\right)$, we consider it here as a mapping on the reference domain, i.e. from $W_{0}\left(D_{0}\right)$ into $W_{1}\left(D_{0}\right)$. It will be one of the central points of this section to make the connection between these two perspectives sound.

In view of Section 3.2 we note in that the operator $T$ introduced in (3.9) can be also be written in terms of the shape parameter $\theta$. We have in particular

$$
T\left(\partial D_{\theta}\right)=(S+A)^{-1}(\theta) f(\theta) .
$$

The aim of this section is to establish Fréchet differentiability with respect to the shape parameter $\theta$ for the solution $u_{\theta}$ in its representation given in (3.35). Using the foundations from Section 3.3 on Fréchet differentiability we proceed by examining the operators

$$
\left(S_{i}(\theta) \varphi\right)(x):=\int_{\Gamma} \Phi_{i, \theta}(x, y) \varphi(y) d s(y), \quad x \in \Sigma_{i}, \varphi \in C^{*}(\Gamma),
$$

and

$$
\left(K_{i}(\theta) \psi\right)(x):=\int_{\partial D_{0}} \frac{\partial \Phi_{i, \theta}(x, y)}{\partial \nu_{\theta}(y)} \psi(y) J_{\theta}(y) d s(y), \quad x \in \Sigma_{i}, \psi \in C\left(\partial D_{0}\right),
$$

where the domain of evaluation $\Sigma_{i}$ is either $\partial D_{0}, \Gamma$, or $\Omega$.

To obtain the sensitivity result, we demonstrate that (3.39) and (3.40) satisfy the assumptions of Theorem 3.24. Moreover, we show that the operators coincide with the potential operators defined in (1.16) and (1.21) if $\Sigma_{i}=\Omega$. In the cases where $\Sigma_{i} \in\left\{\partial D_{0}, \Gamma\right\}$ we show that (3.40) and (3.39) coincide with the boundary integral operators that occur in the system of integral equations (2.36) associated with the geometry $\left(\Gamma, D_{\theta}\right)$. 
We start with the most interesting operator, namely with the double-layer potential operator for the case where the image domain and the domain of integration coincide.

Theorem 3.28. The double-layer potential operator

$$
\int_{\partial D_{\theta}} \frac{\partial \Phi(x, y)}{\partial \nu(y)} \psi(y) d s(y), \quad x \in \partial D_{\theta}
$$

regarded as a mapping $F_{K D}: U \rightarrow L\left(C\left(\partial D_{0}\right), C\left(\partial D_{0}\right)\right)$ satisfies the assumptions of Theorem 3.24. Hence, it is continuously Fréchet differentiable with respect to the parameter $\theta$.

Proof. We start with re-writing (3.41) such that we integrate over the boundary of the fixed reference domain $D_{0}$. We find that

$$
F_{K D}(\theta)=\int_{\partial D_{0}} \frac{\left\langle\nu_{\theta}\left(y_{\theta}\right), x_{\theta}-y_{\theta}\right\rangle}{\left|x_{\theta}-y_{\theta}\right|^{2}} \psi\left(y_{\theta}\right) J_{\theta}(y) d s(y), \quad x \in \partial D_{0},
$$

where $x_{\theta}:=I_{\theta}(x), y_{\theta}:=I_{\theta}(y), \nu_{\theta}\left(y_{\theta}\right)$ denotes the unit normal at $y_{\theta}$ with respect to $\partial D_{\theta}$ and $J_{\theta}(y)=d s\left(y_{\theta}\right)(d s(y))^{-1}$ for $y \in \partial D_{0}$.

We notice that $F_{K D}(\theta)$ coincides with the 2 D-analogue of the expression $K[r] \varphi$ (cf. formula (2.1) of [58]) for the case of $h_{2} \equiv 0$ and $h_{3} \equiv 1$, which are clearly analytic. Next we observe that Lemma 1 of [58] remains valid in two dimensions with exactly the same proof. In particular, it follows that (3.41) satisfies the assumptions (i) and (iv) of Theorem 3.24. From the properties of the doublelayer potential (see [36], Theorem 4.15) it follows that the kernel of (3.42) can be continuously extended for $x=y$. Hence it is integrable and (3.42) is an element of $L\left(C\left(\partial D_{0}\right), C\left(\partial D_{0}\right)\right)$ For the Fréchet derivative of the kernel we compute

$$
\begin{aligned}
\left(\frac{\partial \Phi(x, y)}{\partial \nu(y)}\right)^{\prime}[\theta ; h]= & \frac{\left\langle\nu_{\theta}\left(y_{\theta}\right), h(x)-h(y)\right\rangle}{\left|x_{\theta}-y_{\theta}\right|^{2}}+\frac{\left\langle\nu_{z}(h(y)), x_{\theta}-y_{\theta}\right\rangle}{\left|x_{\theta}-y_{\theta}\right|^{2}} \\
& -2 \frac{\left\langle\nu_{\theta}\left(y_{\theta}\right), x_{\theta}-y_{\theta}\right\rangle\left\langle h(x)-h(y), x_{\theta}-y_{\theta}\right\rangle}{\left|x_{\theta}-y_{\theta}\right|^{4}}
\end{aligned}
$$

for $x, y \in \partial D_{0}$ with $x \neq y$, where

$$
\nu_{z}(h(y)):=\frac{\left[\frac{d}{d \tau} h(y)\right]^{\perp}}{\left|\frac{d}{d \tau} I_{\theta}(y)\right|}, \quad y \in \partial D_{0},
$$

for a smooth parametrization $z$ of $\partial D_{0}$. While the first and last term are obvious from differentiating $x_{\theta}-y_{\theta}$ in the numerator and denominator, the second term that stems from differentiating $\nu_{\theta}\left(y_{\theta}\right)$ requires some additional justification. 
We recall that the double-layer potential in its parametrized form reads

$$
\int_{a}^{b} \frac{\left.\left\langle\left[\left(I_{\theta} \circ z\right)^{\prime}(\tau)\right)\right]^{\perp}, I_{\theta}(z(t))-I_{\theta}(z(\tau))\right\rangle}{\left|I_{\theta}(z(t))-I_{\theta}(z(\tau))\right|^{2}} \psi\left(I_{\theta}(z(\tau))\right) d \tau
$$

for any $(b-a)$-periodic, smooth parametrization $z$. We observe that in this representation the normal $\nu(y)$ is replaced by an expression depending linearly on $\theta$. Hence the Fréchet derivative of this term is given by the same expression applied to the perturbation $h$. Using (3.44) and undoing the parametrization finally yields (3.43).

Integrability and the mapping property (iii) of Theorem 3.24 now follow easily. This is due to (3.43) being well-defined and continuous for $x \neq y$. Furthermore, Lemma 1 of [58] states that the singularity at $x=y$ is not increasing when taking the Fréchet derivative. Hence, (3.43) has a continuous extension for $x=y$.

Therefore, (3.41) satisfies all assumptions of Theorem 3.24 and is by virtue of the same theorem continuously Fréchet differentiable with respect to the shape parameter $\theta$.

We emphasize here that Theorem 3.28 holds regardless of the number of connected components of $D_{0}$ as long as their number stays finite and each component is bounded, simply connected and of class $C^{2}$. Although we have not been explicit in the notation, we mention that each component is thought of as being perturbed independently of the others. Hence, in the case where $D_{0}$ consists of several connected components, we think of a differentiation direction $h$ to be given in the form

$$
h=\left(h_{1}, \ldots, h_{n}\right), \quad h_{i} \in U^{m_{i}, \eta_{i}}\left(D_{i, 0}\right), i=1, \ldots, n .
$$

This interpretation of elements $h \in U$ in the case where $D_{0}$ consists of several connected components is crucial for the numerical realization as it enables an easy deduction of the Frechet derivatives for the operators interacting between the components. It turns out that the kernels (3.43) just need to be splitted according to the components on which $h_{i}$ is defined.

In principle, this splitting of the kernels can be interpreted as considering Fréchet diffrentiability for an operator being defined on one component for which the boundary is varying, and which is mapping to the boundary of another component where the boundary is assumed to be fixed, and vice versa. In this sense, the result of Theorem 3.30 is not too surprising, but we point out here that the result is different to the one above as we equip the image domain of the double-layer operator with a stronger norm.

For numerical purposes we are interested in the value of the kernel (3.43) of the operator $F_{K D}$ for $x=y$. 
Lemma 3.29. Consider the double-layer potential operator as defined in (3.41). Then the following holds:

1. For a general perturbation $h$ the value of $\left(\frac{\partial \Phi(x, y)}{\partial \nu(y)}\right)^{\prime}[\theta ; h] d s(y)$ for $x=y$ is given by

$$
\begin{aligned}
& \left(\frac{\left\langle\left[z^{\prime}(\tau)\right]^{\perp}, \widetilde{h}^{\prime \prime}(\tau)\right\rangle+\left\langle\left[\widetilde{h}^{\prime}(\tau)\right]^{\perp}, z^{\prime \prime}(\tau)\right\rangle}{2\left|z^{\prime}(\tau)\right|^{2}}\right. \\
& \left.-\frac{\left\langle\left[z^{\prime}(\tau)\right]^{\perp}, z^{\prime \prime}(\tau)\right\rangle\left\langle\left[z^{\prime}(\tau)\right]^{\perp},\left[\widetilde{h}^{\prime}(\tau)\right]^{\perp}\right\rangle}{\left|z^{\prime}(\tau)\right|^{4}}\right) d \tau
\end{aligned}
$$

for $x=z(\tau)$, where $z$ is a smooth, periodic parametrization of $\partial D_{\theta}$.

In (3.45) we have set $\widetilde{h}:=h \circ I_{\theta}^{-1} \circ z$.

2. For a normal perturbation, i.e. where $h$ is such that $\widetilde{h}=q\left[z^{\prime}\right]^{\perp}$ for a smooth, periodic parametrization $z$ of $\partial D_{\theta}$ and a smooth scalar function $q$ of the same periodicity, the value of $\left(\frac{\partial \Phi(x, y)}{\partial \nu(y)}\right)^{\prime}[\theta ; h] d s(y)$ for $x=y$ is given by

$$
\left(\frac{1}{2} q^{\prime \prime}(\tau)+\frac{1}{2}\left[q(\tau) \frac{\left\langle z^{\prime}(\tau), z^{\prime \prime}(\tau)\right\rangle}{\left|z^{\prime}(\tau)\right|^{2}}\right]^{\prime}\right) d \tau, \quad x=z(\tau) .
$$

3. If, furthermore, $\partial D_{\theta}$ is parametrized by arclength, i.e. the parametrization satisfies $\left\|z^{\prime}\right\|=1$, the value of $\left(\frac{\partial \Phi(x, y)}{\partial \nu(y)}\right)^{\prime}[\theta ; h] d s(y)$ for $x=y$ is given by

$$
\frac{1}{2} q^{\prime \prime}(\tau) d \tau, \quad x=z(\tau) \text {. }
$$

Proof. We assume that $\partial D_{\theta}$ is given in parametrized form, i.e. we suppose that for some $z:[0,2 \pi) \rightarrow \mathbb{R}^{2}$ we have $\partial D_{\theta}=z([0,2 \pi))$. Then we can express (3.43) for $x \neq y$ in terms of the parametrization $z$, in particular

$$
\begin{aligned}
\left(\frac{\partial \Phi(x, y)}{\partial \nu(y)}\right)^{\prime}[\theta ; h] d s(y) & \\
= & \left(\frac{\left\langle\left[z^{\prime}(\tau)\right]^{\perp}, \widetilde{h}(t)-\widetilde{h}(\tau)\right\rangle+\left\langle\left[\widetilde{h}^{\prime}(\tau)\right]^{\perp}, z(t)-z(\tau)\right\rangle}{|z(t)-z(\tau)|^{2}}\right. \\
& \left.-2 \frac{\left\langle\left[z^{\prime}(\tau)\right]^{\perp}, z(t)-z(\tau)\right\rangle\langle\widetilde{h}(t)-\widetilde{h}(\tau), z(t)-z(\tau)\rangle}{|z(t)-z(\tau)|^{4}}\right) d \tau,
\end{aligned}
$$


where $\widetilde{h}$ is defined as in the statement of the lemma.

We proceed in three steps, determining first the value of the kernel for $t=\tau$ for a general smooth perturbation $\widetilde{h}$, checking secondly that (3.46) coincides with (3.45), when we know that $\widetilde{h}$ points in the direction of the normal to the boundary, and thirdly using the the properties of parametrization by arclength to obtain (3.47).

We begin the first step by using Taylor expansions on the various terms in the numerators and denominators. We have

$$
z(t)-z(\tau)=(t-\tau) \int_{0}^{1} z^{\prime}(\tau+\lambda(t-\tau)) d \lambda
$$

as well as

$$
\begin{aligned}
z(t)-z(\tau)= & z^{\prime}(\tau)(t-\tau)+\frac{z^{\prime \prime}(\tau)}{2}(t-\tau)^{2} \\
& +\frac{(t-\tau)^{3}}{2} \int_{0}^{1}(1-\lambda)^{2} z^{(3)}(\tau+\lambda(t-\tau)) d \lambda
\end{aligned}
$$

and an expression similar to (3.50) for $\widetilde{h}$. Furthermore we observe that the scalar product is invariant, if the operation $[\cdot]^{\perp}$ is applied to both arguments. This leads to

$$
\left\langle\left[z^{\prime}(\tau)\right]^{\perp}, \widetilde{h}^{\prime}(\tau)\right\rangle=-\left\langle\left[\widetilde{h}^{\prime}(\tau)\right]^{\perp}, z^{\prime}(\tau)\right\rangle,
$$

because $\left[[\cdot]^{\perp}\right]^{\perp}=-i d$. So we see by plugging (3.50) for $z$ and $\tilde{h}$ into the numerator of the first term of (3.48) that it reduces to

$$
\frac{(t-\tau)^{2}}{2}\left(\left\langle\left[z^{\prime}(\tau)\right]^{\perp}, \widetilde{h}^{\prime \prime}(\tau)\right\rangle+\left\langle\left[\widetilde{h}^{\prime}(\tau)\right]^{\perp}, z^{\prime \prime}(\tau)\right\rangle\right)+\mathcal{O}\left((t-\tau)^{3}\right)
$$

using (3.51). Similarly, plugging (3.50) for $z$ and $\widetilde{h}$ into the numerator of the second term of (3.48) leads to

$$
\frac{(t-\tau)^{4}}{2}\left\langle\left[z^{\prime}(\tau)\right]^{\perp}, z^{\prime \prime}(\tau)\right\rangle\left\langle\left[z^{\prime}(\tau)\right]^{\perp},\left[\widetilde{h}^{\prime}(\tau)\right]^{\perp}\right\rangle+\mathcal{O}\left((t-\tau)^{5}\right)
$$

since $\left\langle\left[z^{\prime}(\tau)\right]^{\perp}, z^{\prime}(\tau)\right\rangle=0$ and $\left\langle\widetilde{h}^{\prime}(\tau), z^{\prime}(\tau)\right\rangle=\left\langle\left[z^{\prime}(\tau)\right]^{\perp},\left[\widetilde{h}^{\prime}(\tau)\right]^{\perp}\right\rangle$.

Finally, substituting (3.49) into the denominators of (3.48), using the above representations for the numerators and letting $(t-\tau)$ tend to zero, we obtain

$$
\begin{array}{r}
\frac{\left\langle\left[z^{\prime}(\tau)\right]^{\perp}, \widetilde{h}^{\prime \prime}(\tau)\right\rangle+\left\langle\left[\widetilde{h}^{\prime}(\tau)\right]^{\perp}, z^{\prime \prime}(\tau)\right\rangle}{2\left|z^{\prime}(\tau)\right|^{2}} \\
-\frac{\left\langle\left[z^{\prime}(\tau)\right]^{\perp}, z^{\prime \prime}(\tau)\right\rangle\left\langle\left[z^{\prime}(\tau)\right]^{\perp},\left[\widetilde{h}^{\prime}(\tau)\right]^{\perp}\right\rangle}{\left|z^{\prime}(\tau)\right|^{4}}
\end{array}
$$


for the value of the parametrized kernel at $t=\tau$ This proves the first statement and thereby completes the first step of the proof.

For the second step we may assume that $\widetilde{h}$ is of the form $q\left[z^{\prime}\right]^{\perp}$, where $\left[z^{\prime}\right]^{\perp}$ is a vector field pointing into the direction normal to the boundary, and where $q:[0,2 \pi) \rightarrow \mathbb{R}$ is some smooth, $2 \pi$-periodic scalar field. We then compute the first and second derivative of $\widetilde{h}$ as

$$
\begin{aligned}
\widetilde{h}^{\prime}(\tau) & =q^{\prime}(\tau)\left[z^{\prime}(\tau)\right]^{\perp}+q(\tau)\left[z^{\prime \prime}(\tau)\right]^{\perp} \\
\widetilde{h}^{\prime \prime}(\tau) & =q^{\prime \prime}(\tau)\left[z^{\prime}(\tau)\right]^{\perp}+2 q^{\prime}(\tau)\left[z^{\prime \prime}(\tau)\right]^{\perp}+q(\tau)\left[z^{(3)}(\tau)\right]^{\perp} .
\end{aligned}
$$

Substituting this into (3.52) and using $\left[\left[z^{(k)}\right]^{\perp}\right]^{\perp}=-z^{(k)}$ for $k=1,2,3$, we obtain for the numerator of the first term of $(3.52)$

$$
\begin{aligned}
q^{\prime \prime}(\tau)\left\langle\left[z^{\prime}(\tau)\right]^{\perp},\right. & {\left.\left[z^{\prime}(\tau)\right]^{\perp}\right\rangle } \\
& +q^{\prime}(\tau)\left(2\left\langle\left[z^{\prime}(\tau)\right]^{\perp},\left[z^{\prime \prime}(\tau)\right]^{\perp}\right\rangle-\left\langle z^{\prime}(\tau), z^{\prime \prime}(\tau)\right\rangle\right) \\
& +q(\tau)\left(\left\langle\left[z^{\prime}(\tau)\right]^{\perp},\left[z^{(3)}(\tau)\right]^{\perp}\right\rangle-\left\langle z^{\prime \prime}(\tau), z^{\prime \prime}(\tau)\right\rangle\right) .
\end{aligned}
$$

Similarly, we calculate the numerator of the second term of (3.52)

$$
\begin{aligned}
& -q^{\prime}(\tau)\left\langle\left[z^{\prime}(\tau)\right]^{\perp}, z^{\prime \prime}(\tau)\right\rangle\left\langle\left[z^{\prime}(\tau)\right]^{\perp}, z^{\prime}(\tau)\right\rangle \\
& -q(\tau)\left\langle\left[z^{\prime}(\tau)\right]^{\perp}, z^{\prime \prime}(\tau)\right\rangle\left\langle\left[z^{\prime}(\tau)\right]^{\perp}, z^{\prime \prime}(\tau)\right\rangle,
\end{aligned}
$$

which due to $\left\langle\left[z^{\prime}(\tau)\right]^{\perp}, z^{\prime}(\tau)\right\rangle=0$ is equal to

$$
-q(\tau)\left(\left\langle\left[z^{\prime}(\tau)\right]^{\perp}, z^{\prime \prime}(\tau)\right\rangle\right)^{2}
$$

As $\frac{z^{\prime}}{\left|z^{\prime}\right|}$ and $\frac{\left[z^{\prime}\right]^{\perp}}{\left|z^{\prime}\right|}$ form an orthonormal basis of $\mathbb{R}^{2}$ the identity

$$
\left\langle z^{\prime \prime}(\tau), z^{\prime \prime}(\tau)\right\rangle=\frac{\left\langle z^{\prime}(\tau), z^{\prime \prime}(\tau)\right\rangle^{2}}{\left|z^{\prime}(\tau)\right|^{2}}+\frac{\left\langle\left[z^{\prime}(\tau)\right]^{\perp}, z^{\prime \prime}(\tau)\right\rangle^{2}}{\left|z^{\prime}(\tau)\right|^{2}}
$$

holds and (3.53) thereby is equal to

$$
q(\tau)\left(\left\langle z^{\prime}(\tau), z^{\prime \prime}(\tau)\right\rangle^{2}-\left\langle z^{\prime \prime}(\tau), z^{\prime \prime}(\tau)\right\rangle\left|z^{\prime}(\tau)\right|^{2}\right) .
$$

With this choice for $\widetilde{h}$ it follows that (3.52) reads

$$
\begin{aligned}
\frac{1}{2} q^{\prime \prime}(\tau)+\frac{1}{2} q^{\prime}(\tau) \frac{\left\langle z^{\prime}(\tau), z^{\prime \prime}(\tau)\right\rangle}{\left|z^{\prime}(\tau)\right|^{2}} & \\
& +\frac{1}{2} q(\tau)\left(\frac{\left\langle z^{\prime}(\tau), z^{(3)}(\tau)\right\rangle+\left\langle z^{\prime \prime}(\tau), z^{\prime \prime}(\tau)\right\rangle}{\left|z^{\prime}(\tau)\right|^{2}}-2 \frac{\left\langle z^{\prime}(\tau), z^{\prime \prime}(\tau)\right\rangle^{2}}{\left|z^{\prime}(\tau)\right|^{4}}\right)
\end{aligned}
$$


Now it follows that (3.46) coincides with the above as can be seen by differentiating the second summand of (3.46) term by term.

For the last statement of the lemma we just observe that if $z$ is a parametrization of $\partial D_{\theta}$ by arclength it satisfies $\left\langle z^{\prime \prime}(\tau), z^{\prime}(\tau)\right\rangle=0$. Consequently, the second summand of (3.46) vanishes identically and we obtain (3.47).

With the previous lemma we have finished the study of the differentiability of the double-layer potential operator (3.41) that forms the central ingredient for the sensitivity results we are going to present shortly. Nevertheless, (3.36) - (3.38) involve several other boundary integral operators which we will treat now in the same fashion.

Theorem 3.30. The double-layer potential operator

$$
\int_{\partial D_{\theta}} \frac{\partial \Phi(x, y)}{\partial \nu(y)} \psi(y) d s(y), \quad x \in M
$$

where $M$ is a compact subset of the complement of $\operatorname{supp}(\theta)$, regarded as a mapping $F_{K D M}: U \rightarrow L\left(C\left(\partial D_{0}\right), C^{2}(M)\right)$ satisfies the assumptions of Theorem 3.24. Hence, it is continuously Fréchet differentiable with respect to the parameter $\theta$.

Proof. First, we again re-write (3.55) in terms of the fixed reference domain $D_{0}$ as

$$
F_{K D M}(\theta)=\int_{\partial D_{0}} \frac{\left\langle\nu_{\theta}\left(y_{\theta}\right), x-y_{\theta}\right\rangle}{\left|x-y_{\theta}\right|^{2}} \psi\left(y_{\theta}\right) J_{\theta}(y) d s(y), \quad x \in M,
$$

noting that since $M \cap \partial D_{\theta}=\emptyset$ for any $\theta \in U$ the kernel of (3.56) is smooth in both variables. Hence, the Fréchet derivatives of the kernel with respect to $\theta$ up to the second order exist and the regularity with respect to $x$ and $y$ is determined by the regularity of $\theta, h, \partial D_{0}$ and $M$. Since $\theta$ and $h$ vanish on $M$, the first order Fréchet derivative for $x \in M$ and $y \in \partial D_{0}$ reads

$$
\begin{aligned}
\left(\frac{\partial \Phi(x, y)}{\partial \nu(y)}\right)^{\prime}[\theta ; h]= & -\frac{\left\langle\nu_{\theta}\left(y_{\theta}\right), h(y)\right\rangle}{\left|x-y_{\theta}\right|^{2}}+\frac{\left\langle\nu_{z}(h(y)), x-y_{\theta}\right\rangle}{\left|x-y_{\theta}\right|^{2}} \\
& +2 \frac{\left\langle\nu_{\theta}\left(y_{\theta}\right), x-y_{\theta}\right\rangle\left\langle h(y), x-y_{\theta}\right\rangle}{\left|x-y_{\theta}\right|^{4}}
\end{aligned}
$$

where $\nu_{z}(h(y))$ is again understood as in (3.44). Due to the previous observations we see that in particular (3.57) is continuous for all $x, y$. Consequently, assumptions (i) and (ii) of Theorem 3.24 are satisfied. Furthermore, the compactness of the perturbed boundary $\partial D_{\theta}$ and its being disjoint from $M$, which we assume to 
be at least $C^{2}$-regular imply that $F_{K D M}(\theta)$ and its first order Fréchet derivative map boundedly into twice continuously differentiable functions on $M$ such that also assumption (iii) of Theorem 3.24 is satisfied. Finally, assumption (iv) of Theorem 3.24 is checked by employing the estimates

$$
\begin{aligned}
& \left|x-y_{\theta}\right|=\left|x_{\theta}-y_{\theta}\right| \leq c_{1}|x-y|, \quad x \in M, y \in \partial D_{0}, \\
& \left|x-y_{\theta}\right|=\left|x_{\theta}-y_{\theta}\right| \geq c_{2}|x-y|, \quad x \in M, y \in \partial D_{0}
\end{aligned}
$$

on the second order Fréchet derivatives, which are valid for $M$ and $\partial D_{0}$ being submanifolds on $\mathbb{R}^{2}$ as has been stated in [58].

The above theorem includes the cases where the double-layer potential maps either onto the arc $\Gamma$ or to the domain $\Omega$ as it is understood in (3.38). We finish the examination of the sensitivity properties for the layer potentials by considering the single-layer potential defined on the $\operatorname{arc} \Gamma$.

Lemma 3.31. The single-layer potential

$$
\int_{\Gamma} \Phi(x, y) \varphi(y) d s(y), \quad x \in M
$$

where $M$ is a compact subset of the complement of the support of $\theta$, regarded as a mapping $F_{S \Gamma M}: U \rightarrow L\left(C^{*}(\Gamma), C^{2}(M)\right)$ is continuously Fréchet differentiable with respect to variations of the boundary of $D$ that are expressed in the shape parameter $\theta$.

Proof. We note that $(\Gamma \cup M) \cap \operatorname{supp}(\theta)=\emptyset$. Hence, (3.60) is constant with respect to $\theta$ and thus, it is Fréchet differentiable with Fréchet derivative being the zero operator $0 \in L\left(C^{*}(\Gamma), C^{2}(M)\right)$.

Similarly to Theorem 3.30, where we have considered $F_{K D M}$, Lemma 3.31 also applies in two cases, namely in the one where $M=\Gamma$ and in the case $M=\Omega$, where $\Omega$ is understood as in (3.38).

Theorem 3.32. The single-layer potential

$$
\int_{\Gamma} \Phi(x, y) \varphi(y) d s(y), \quad x \in \partial D_{\theta}
$$

regarded as a mapping $F_{S \Gamma D}: U \rightarrow L\left(C^{*}(\Gamma), C\left(\partial D_{0}\right)\right)$ satisfies the assumptions of Theorem 3.24. Hence, it is continuously Fréchet differentiable with respect to the parameter $\theta$. 
Proof. First, we note that we can express $F_{\Gamma D}$ in terms of the reference domain by exchanging $x_{\theta}$ for $x$ in (3.61). As before the kernel is analytic in both variables since $\Gamma \cap \partial D_{\theta}=\emptyset$. Thus, it is Fréchet differentiable, and the kernel together with its Fréchet derivatives are as smooth as $\theta, h, \partial D_{0}$ and $\Gamma$. The first order Fréchet derivative for $x \in \partial D_{0}$ and $y \in \Gamma$ reads

$$
(\Phi(x, y))^{\prime}[\theta ; h]=-\frac{\left\langle x_{\theta}-y, h(x)\right\rangle}{\left|x_{\theta}-y\right|^{2}} .
$$

Now we immediately see that assumptions (i) to (iii) of Theorem 3.24 are satisfied due to the required mapping properties. Assumption (iv) can again be checked using the estimates (3.58) and (3.59) with the roles of $x$ and $y$ interchanged.

Now we have settled the question of Fréchet differentiability for all the operators occuring in (3.35). Hence, we can state the following theorem which establishes the differentiable dependence of the solution $u_{\theta}$ and the operator $T$ from (3.9) on the shape parameter $\theta$. This constitutes the central result of this section.

Theorem 3.33. Let $\left(\Gamma, D_{0}\right)$ be an admissible geometry for the Boundary Value Problem 2.1 and let $\theta \in U$. Then the following statements hold:

1. The unique solution $\Psi_{\theta}$ to the system of integral equations (2.36) for the geometry $\left(\Gamma, D_{\theta}\right)$, regarded as dependent on the shape parameter $\theta$, is Fréchet differentiable with respect to $\theta$.

2. The unique solution $u_{\theta}$ to Problem 2.1 for the geometry $\left(\Gamma, D_{\theta}\right)$, regarded as dependent on the shape parameter $\theta$, is also Fréchet differentiable with respect to $\theta$.

Proof. Firstly, we consider the underlying system of integral equations (2.36) for the geometry $\left(\Gamma, D_{\theta}\right)$. According to Theorem 2.10 the system is uniquely solvable for any right hand side from the appropriate spaces, and in particular for the right hand side given in $(2.33)$ with a solution $\Psi_{\theta}=\left(\widetilde{\varphi}_{\theta}, \psi_{1, \theta}, \ldots, \psi_{n, \theta}\right) \in W_{0}\left(D_{\theta}\right)$. Defining

$$
\Psi_{0}:=\left(\widetilde{\varphi}_{\theta}, \psi_{1,0}, \ldots, \psi_{n, 0}\right) \in W_{0}\left(D_{0}\right)
$$

with $\psi_{i, 0}:=\psi_{i, \theta} \circ I_{\theta}$, we can express the solution to (2.36) in terms of a function defined on the boundary of the fixed reference domain $D_{0}$. Now the results of Theorem 3.28, Theorem 3.30, Lemma 3.31 and Theorem 3.32 show that the system of integral equations (2.36) for the Boundary Value Problem 2.1 and

$$
\left(\begin{array}{cc}
\widetilde{F}_{S \Gamma \Gamma}(\theta) & F_{K D \Gamma}(\theta) \\
2 q \widetilde{F}_{S \Gamma D}(\theta) & \left(\operatorname{Id}+2 q F_{K D}\right)(\theta)
\end{array}\right) \Psi_{0}=\left(\begin{array}{c}
-F_{S \Gamma \Gamma}(\theta)\left(\frac{I}{|\Gamma|}\right) \\
-2 q F_{S \Gamma D}(\theta)\left(\frac{I}{|\Gamma|}\right)
\end{array}\right)
$$


are equivalent in the sense that each solution to (2.36) induces a solution to (3.63) via the above definition of $\Psi_{0}$, and conversely each solution to (3.63) defines a solution $\Psi_{\theta}$ to $(2.36)$ via

$$
\Psi_{\theta}:=\left(\widetilde{\varphi}_{\theta}, \psi_{1,0} \circ I_{\theta}^{-1}, \ldots, \psi_{n, 0} \circ I_{\theta}^{-1}\right) .
$$

In (3.63), the operators $\widetilde{F}_{S \Gamma D}(\theta)$ and $\widetilde{F}_{S \Gamma \Gamma}(\theta)$ are given by

$$
\begin{aligned}
\widetilde{F}_{S \Gamma D}(\theta)(\varphi) & :=F_{S \Gamma D}(\theta)(M \varphi)+\frac{1}{|\Gamma|} \int_{\Gamma} \varphi d s \\
\widetilde{F}_{S \Gamma \Gamma}(\theta)(\varphi) & :=F_{S \Gamma \Gamma}(\theta)(M \varphi)+\frac{1}{|\Gamma|} \int_{\Gamma} \varphi d s .
\end{aligned}
$$

As a consequence of the equivalence between (3.63) and (2.36), we deduce from Theorem 2.10 that (3.63) has a unique solution. Hence we can consider the mapping $(S+A)^{-1}(\theta) f(\theta): U \rightarrow W_{0}\left(D_{0}\right)$ given by

$$
\left(\begin{array}{cc}
\widetilde{F}_{S \Gamma \Gamma}(\theta) & F_{K D \Gamma}(\theta) \\
2 q \widetilde{F}_{S \Gamma D}(\theta) & \left(\mathrm{Id}+2 q F_{K D}\right)(\theta)
\end{array}\right)^{-1}\left(\begin{array}{c}
-F_{S \Gamma \Gamma}(\theta)\left(\frac{I}{|\Gamma|}\right) \\
-2 q F_{S \Gamma D}(\theta)\left(\frac{I}{|\Gamma|}\right)
\end{array}\right) .
$$

We note that all operators occuring in (3.65) are continuously Fréchet differentiable with respect to the shape parameter $\theta$ due to the results of Theorem 3.28, Theorem 3.30, Lemma 3.31 and Theorem 3.32. The first statement then follows from (3.63) by applying Corollary 3.20, Theorem 3.22 and Lemma 3.21.

For the second statement we also exploit the equivalenvce of (2.36) and (3.63). From Theorem 3.30 and Lemma 3.31 we note that the expression

$$
P\left(\theta, \Psi_{0}\right):=\left(\begin{array}{ll}
F_{K D \Omega}(\theta) & F_{S \Gamma \Omega}(\theta)
\end{array}\right) \Psi_{0}
$$

where $\Psi_{0}$ solves (3.63), coincides with the representation (2.10) for a density $\Psi_{\theta}$ defined by (3.64). Due to the existence theorem (Theorem 2.10) and the above equivalence, (3.66) then defines a solution to the Boundary Value Problem 2.1 for the geometry $\left(\Gamma, D_{\theta}\right)$. Now the Fréchet differentiability of $P$ is a consequence of Theorem 3.30, Lemma 3.31 and Corollary 3.20, and the second statement of the theorem follows from Lemma 3.21 and the first statement.

We conclude this section with two remarks. Firstly, we would like to point out that the form of the Fréchet derivative for $\Psi_{\theta}$ and $u_{\theta}$ can be obtained easily from the general differentiability results of Section 3.3 and from the expressions for the kernels that have been derived in the course of the proofs in this section.

Secondly, we emphasize that in view of the factorization (3.13) from Section 3.2 the first statement of Theorem 3.33 implies the Fréchet differentiability of the operator $T$ given in 3.9 with respect to the shape parameter $\theta$ as we have the correspondence $\Psi_{\theta}=T\left(\partial D_{\theta}\right)$. 


\subsection{A domain sensitivity result for the Geomet- ric Optimization Problem}

In the introduction to the present chapter and again at the end of Section 3.2 we have pointed out that the key to a numerical approximation scheme for a nonlinear optimization problem is the differentiable dependence of the objective functional on the considered parameter, which in our case is the shape parame$\operatorname{ter} \theta$. Moreover, as differentiability implies continuity it is also at the heart of theoretical existence proofs. This section consequently deals with establishing Fréchet differentiability for $F$ with respect to $\theta$. Furthermore, we will derive an existence result for the Geometric Optimization Problem 3.3.

In view of the representation (3.13) from Section 3.2 and of the first statement of Theorem 3.33 from the previous section it only remains to study the operators $P_{\Gamma}$ defined in (3.10), the operator $M_{\gamma}$ defined in (3.11) and the functional $G$ defined in (3.12). We state the according differentiability results in the following lemmas.

Lemma 3.34. The functional $G: C_{2 \pi, e}^{0, \alpha} \rightarrow \mathbb{R}$ given by

$$
G(\psi)=\frac{1}{2} \int_{0}^{\pi}(\psi(t))^{2} d t
$$

is Fréchet-differentiable and the Fréchet derivative in direction $h$ is given by

$$
G^{\prime}[\psi ; h]=\int_{0}^{\pi} \psi(t) h(t) d t .
$$

Proof. The statement simply follows from

$$
\begin{aligned}
G(\psi+h) & =\frac{1}{2} \int_{0}^{\pi}(\psi(t)+h(t))^{2} d t \\
& =G(\psi)+\int_{0}^{\pi} \psi(t) h(t) d t+\frac{1}{2} \int_{0}^{\pi}(h(t))^{2} d t
\end{aligned}
$$

for $\psi, h \in C_{2 \pi, e}^{0, \alpha}$ as

$$
\left|\frac{1}{2} \int_{0}^{\pi}(h(t))^{2} d t\right| \leq \frac{\pi}{2}\|h\|_{C_{2 \pi, e}^{0, \alpha}}^{2} .
$$

Lemma 3.35. The operator $B_{\gamma}: W_{0}\left(D_{0}\right) \rightarrow C_{2 \pi, e}^{0, \alpha}$ defined by

$$
B_{\gamma} \Psi:=\left(M_{\gamma} \circ P_{\Gamma}\right)(\Psi)
$$


where $P_{\Gamma}$ is given by (3.10) and $M_{\gamma}$ is defined as in (3.11), is linear and bounded. The adjoint $B_{\gamma}^{*}: C_{2 \pi, e}^{0, \alpha} \rightarrow W_{0}\left(D_{0}\right)$ of $B_{\gamma}$ with respect to the $L_{2}$-bilinear pairing is given by

$$
B_{\gamma}^{*}(\psi)=\left(\psi-\frac{1}{|\Gamma|} \int_{0}^{\pi} \psi(s) w(\gamma(\cos s)) d s, 0, \ldots, 0\right) .
$$

Furthermore, $B_{\gamma}$ is Fréchet-differentiable and coincides with its Fréchet derivative.

Proof. Linearity and boundedness of $B_{\gamma}$ are obvious from (3.69), (3.10) and (3.11). Hence, Fréchet differentiability of $B_{\gamma}$ and the form of the Fréchet derivative follow directly from Lemma 3.11.

We denote by $\langle\cdot, \cdot\rangle$ the sum of the $L_{2}$ bilinear pairings on $[0, \pi]$ and $C\left(\partial D_{k}\right)$ for $k=1, \ldots, n$. Then we calculate for $\psi \in C_{2 \pi, e}^{0, \alpha}$ and $\left(\varphi_{\Gamma}, \varphi_{1}, \ldots, \varphi_{n}\right) \in W_{0}\left(D_{0}\right)$

$$
\begin{aligned}
\left\langle B_{\gamma}^{*}(\psi),\left(\varphi_{\Gamma}, \varphi_{1}, \ldots, \varphi_{n}\right)\right\rangle & \\
= & \int_{0}^{\pi}\left(\psi(t)-\frac{1}{|\Gamma|} \int_{0}^{\pi} \psi(s) w(\gamma(\cos s)) d s\right) \varphi_{\Gamma}(t) d t \\
& \quad+\sum_{k=1}^{n} \int_{\partial D_{k}} 0 \cdot \varphi_{k}(x) d s(x) \\
= & \int_{0}^{\pi} \psi(t) \cdot\left(\varphi_{\Gamma}(t)-\frac{1}{|\Gamma|} w(\gamma(\cos t)) \int_{0}^{\pi} \varphi_{\Gamma}(s) d s\right) d t \\
= & \left\langle\psi, B_{\gamma}\left(\varphi_{\Gamma}, \varphi_{1}, \ldots, \varphi_{n}\right)\right\rangle_{[0, \pi]}
\end{aligned}
$$

by interchanging the order of integration. This proves that $B_{\gamma}$ and $B_{\gamma}^{*}$ are indeed adjoint with respect to the $L_{2}$ bilinear pairing.

Now we are in the position to combine the previous results in order to obtain the aspired sensitivity result for the functional $F$ of the Geometric Optimization Problem 3.3.

Theorem 3.36. Let the functional $F$ be given by (3.4). Then it is Fréchet differentiable with respect to the shape parameter $\theta$.

Proof. The key observation for the proof is the identity (3.13)

$$
\begin{aligned}
F\left(\partial D_{\theta}\right) & =\left(G \circ M_{\gamma} \circ P_{\Gamma} \circ T\right)\left(\partial D_{\theta}\right) \\
& =\left(G \circ B_{\gamma}\right) \Psi_{\theta} .
\end{aligned}
$$


The statement now just follows from Lemma 3.34, Lemma 3.35 and the first statement of Theorem 3.33 by applying the chain rule given in the second statement of Lemma 3.12. Note that we can indeed apply the chain rule since the proof of Theorem 3.33 reveals that $\Psi_{\theta}$ can be thought of as defined on $W_{0}\left(D_{0}\right)$. Thus, we have got rid of the explicit dependence on $\partial D_{\theta}$ for the operator $P_{\Gamma}$ from (3.10).

With Theorem 3.36 we have finally derived the second central result of this chapter, which establishes a differentiable dependence between the shape parameter and the values of the functional $F$. As Fréchet differentiability implies also continuity for $F$, Theorem 3.36 also provides the key for the following existence result with which we close this chapter.

Theorem 3.37. Let $\Gamma$ be an open arc of class $C^{3}$, and let the parameters $I \neq 0$ as well as $\mu_{e}, \mu_{1}, \ldots, \mu_{n}$ be given. Furthermore, let $D_{0}^{(1)}, \ldots, D_{0}^{(N)}$ be a finite collection of reference domains, and define $U^{m_{i}, \eta_{i}}\left(D_{0}^{(i)}\right)$ according to Definition 3.26. Assume now that $\mathcal{U}_{\text {ad }}$ is given by

$$
\mathcal{U}_{a d}=\bigcup_{i=1}^{N}\left\{I_{\theta}\left(\partial D_{0}^{(i)}\right): \theta \in V_{i}\right\},
$$

where $V_{i}$ is a compact subset of $U^{m_{i}, \eta_{i}}\left(D_{0}^{(i)}\right)$. Then the Geometric Optimization Problem 3.3 is solvable, i.e. there exists an admissible boundary $\partial D_{\theta^{*}} \in \mathcal{U}_{a d}$ such that

$$
F\left(\partial D_{\theta^{*}}\right) \leq F\left(\partial D_{\theta}\right) \quad \text { for all } \partial D_{\theta} \in \mathcal{U}_{a d}
$$

and correspondingly, $u_{\theta^{*}}$ is a solution to the Boundary Value Problem 2.1 for the geometry $\left(\Gamma, \partial D_{\theta^{*}}\right)$.

Proof. Let us consider Problem 3.3 with $\mathcal{U}_{a d}$ given by (3.71). From the factorization (3.13) and the properties of $G$ (see Lemma 3.34) that there exists a minimizing sequence $\left(\partial D_{\theta_{n}}\right)_{n \in \mathbb{N}}$ for $F$, i.e.

$$
\lim _{n \rightarrow \infty} F\left(\partial D_{\theta_{n}}\right)=\inf _{\partial D_{\theta} \in \mathcal{U}_{a d}} F\left(\partial D_{\theta}\right) \geq 0
$$

From the definition of $\mathcal{U}_{a d}$ we obtain that there exists a subsequence for which the shape parameter $\theta_{n_{k}}$ is contained in some $V_{i}$. As $V_{i}$ is compact in $U^{m_{i}, \eta_{i}}\left(D_{0}^{(i)}\right)$, there exists an element $\theta^{*} \in V_{i}$ and a subsequence which converges to $\theta^{*}$ in $V_{i}$. To keep the notation simple we denote the convergent subsequence again by $\theta_{n}$. This means that we have in terms of $\mathcal{U}_{a d}$

$$
\partial D_{\theta_{n}} \rightarrow \partial D_{\theta^{*}}, \quad n \rightarrow \infty
$$


where the convergence is understood in the sense of

$$
\theta_{n} \rightarrow \theta^{*}, \quad n \rightarrow \infty
$$

in $V_{i} \subset U^{m_{i}, \eta_{i}}\left(D_{0}^{(i)}\right)$ with respect to the $C^{2}$-norm. Now the sensitivity result Theorem 3.33 yields that the we also have

$$
u_{\theta_{n}} \rightarrow u_{\theta^{*}}, \quad n \rightarrow \infty
$$

as Fréchet differentiability implies continuity. Similarly the Fréchet differentiability of $F$ with respect to $\theta$ (see Theorem 3.36) implies that we have convergence

$$
F\left(\partial D_{\theta_{n}}\right) \rightarrow F\left(\partial D_{\theta_{*}}\right), \quad n \rightarrow \infty
$$

As $\left(\partial D_{\theta_{n}}\right)$ is a subsequence of a minimizing sequence, we have that

$$
F\left(\partial D_{\theta^{*}}\right)=\lim _{n \rightarrow \infty} F\left(\partial D_{\theta_{n}}\right)=\inf _{\partial D_{\theta} \in \mathcal{U}_{a d}} F\left(\partial D_{\theta}\right)
$$

Hence, $\partial D_{\theta^{*}}$ is a minimizer for the Geometric Optimization Problem 3.3 and $u_{\theta^{*}}$ is a solution to the Boundary Value Problem 2.1 for the geometry $\left(\Gamma, D_{\theta^{*}}\right)$.

We finish the theoretic considerations with the remark that the existence result of Theorem 3.37 is still of rather abstract nature as it is not at all obvious how to realize $\mathcal{U}_{a d}$ as the set of perturbed boundaries $I_{\theta}\left(\partial D_{0}^{(i)}\right)$ of finitely many reference domains $D_{0}^{(i)}$. 


\section{Chapter 4}

\section{Numerical treatment}

In this chapter we present approximation schemes to both the Boundary Value Problem of Chapter 2 and the Geometric Optimization Problem of Chapter 3. We will see in the first section that the approximation scheme for the solution to the Boundary Value Problem 2.1 emerges from the constructive nature of proving the existence of a solution in Section 2.3. The Geometric Optimization Problem 3.3 will be treated in the second and third section using two conceptually antithetic approaches. In the second section we will present an approach using explicit boundary representation by restricting the boundaries $\partial D_{i}, i=1, \ldots, n$, to be parametrizable in the form

$$
\partial D_{i}=\left\{x \in \mathbb{R}^{2}: x=x_{0, i}+r_{i}(t)\left(\begin{array}{c}
\cos t \\
\sin t
\end{array}\right), t \in[0,2 \pi)\right\}
$$

with radial functions $r_{i} \in C_{2 \pi}^{2}$. The optimization is then realized through a steepest descent approach on a finite dimensional subspace. In the third section we seek to find a solution to Problem 3.3 using implicit boundary representation. This approach is based on so-called level set methods, where the boundaries of the geometric objects to be optimized are carried along implicitly as the 0-level sets of a higher dimensional function $\phi$, i.e. for a fixed $t \geq 0$ we have

$$
\partial D=\left\{x \in \mathbb{R}^{2}: \phi(x, t)=0\right\}
$$

In this approach the optimization is realized through advecting the higher dimensional level set function $\phi$ in artificial time $t$ in an appropriate fashion. 


\subsection{An exponentially convergent approximation scheme for the solution to the Boundary Value Problem}

The aim of this section is to describe an approximation scheme to the solution of the Boundary Value Problem 2.1 from the second chapter. The approximation scheme will be based on the representation of the solution as a combination of double-layer potentials over the boundary of $D$ and a single-layer potential over the arc $\Gamma$ as has been done in Section 2.3. We will start out from the representation (2.10) and seek approximate solutions using a combination of collocation and quadrature methods on the transformed system of integral equations (2.36). So let us begin by stating rigorously, what we understand by the above.

Consider the spaces $\widetilde{W}_{0}:=C_{2 \pi, e}^{0, \alpha} \times \otimes_{i=1}^{n} C_{2 \pi}$ and $\widetilde{W}_{1}:=C_{2 \pi, e}^{1, \alpha} \times \otimes_{i=1}^{n} C_{2 \pi}$ as well as the finite dimensional subspace $\widetilde{W}^{(m)}:=T_{m_{0}, e} \times \otimes_{i=1}^{n} T_{m_{i}}$ with $m:=\left(m_{0}, \ldots, m_{n}\right)$, where

$$
T_{m_{i}}:=\left\{\sum_{k=0}^{m_{i}} \alpha_{k, i} \cos (k t)+\sum_{k=1}^{m_{i}-1} \beta_{k, i} \sin (k t): \alpha_{k, i}, \beta_{k, i} \in \mathbb{R}, t \in \mathbb{R}\right\}
$$

is the space of trigonometric polynomials of degree $\leq m_{i}$, and where

$$
T_{m_{0}, e}:=\left\{\sum_{k=0}^{m_{0}} \alpha_{k, 0} \cos (k t): \alpha_{k, 0} \in \mathbb{R}, t \in \mathbb{R}\right\}
$$

is the space of even trigonometric polynomials of degree $\leq m_{0}$. We observe that $\widetilde{W}^{(m)}$ is a subspace of $\widetilde{W}_{0}$ as well as of $\widetilde{W}_{1}$. It is of dimension

$$
M:=1+m_{0}+\sum_{i=1}^{n} 2 m_{i}
$$

and has the property that in the limit $m \rightarrow \infty$ it is dense both in $\widetilde{W}_{0}$ and $\widetilde{W}_{1}$. We now equip $\widetilde{W}^{(m)}$ with points

$$
\begin{aligned}
x_{k}^{(0)} \in[0, \pi], & k=0, \ldots, m_{0}, \\
x_{k}^{(i)} \in[0,2 \pi), & i=1, \ldots, n, k=1, \ldots, m_{i},
\end{aligned}
$$

such that $\widetilde{W}^{(m)}$ is unisolvent with respect to these points. (In the actual implementation we have chosen equidistantly spaced collocation points $x_{k}^{(0)}:=k \frac{\pi}{m_{0}}$ for $k=0, \ldots, m_{0}$, and $x_{k}^{(i)}:=(k-1) \frac{2 \pi}{m_{i}}$ for $i=1, \ldots, n$ and $k=1, \ldots, m_{i}$, which fit our needs perfectly.) 
Next we note that by using smooth, regular, $2 \pi$-periodic parametrizations $z_{i}$ of the boundaries $\partial D_{i}$ for $i=1, \ldots, n$, we can consider the operator equation

$$
(S+A) \Psi=f
$$

as given in (2.36) to hold in $\widetilde{W}_{1}$ for a density in $\widetilde{W}_{0}$, where we use the identification

$$
\widehat{\psi}_{i}(t)=\psi_{i}\left(z_{i}(t)\right), \quad t \in \mathbb{R},
$$

for $\widehat{\psi}_{i} \in C_{2 \pi}$ and $\psi_{i} \in C\left(\partial D_{i}\right)$. We observe that in this sense the subspace $\widetilde{W}^{(m)}$ is also dense in $W_{0}$ and $W_{1}$ for $m \rightarrow \infty$. Then the collocation method is given by the following.

Problem 4.1. Find $\Psi^{(m)} \in \widetilde{W}^{(m)}$ satisfying the parametrized version

$$
(\widehat{S}+\widehat{A}) \Psi^{(m)}=\widehat{f}
$$

of (2.36) at the so-called collocation points $\left(x_{k}^{(i)}\right)$ given by (4.1).

In (4.2) the operator $\widehat{S}$ differs from the operator $S$ given in (2.31) only in the sense that the identities have to be understood as identities on $C_{2 \pi}$, and not as mappings on $C\left(\partial D_{i}\right)$ anymore. Similarly, the right-hand side $\widehat{f}$ emerges from $f$ given in (2.33) through

$$
\widehat{f}=\left(\begin{array}{llll}
-\widetilde{S}^{\Gamma}\left(\frac{I}{|\Gamma|}\right) & -2 q_{1} S_{1}^{\Gamma, D}\left(\frac{I}{|\Gamma|}\right)\left(z_{1}(\cdot)\right) & \cdots & \left.-2 q_{n} S_{n}^{\Gamma, D}\left(\frac{I}{|\Gamma|}\right)\left(z_{n}(\cdot)\right)\right)^{T} .
\end{array}\right.
$$

Finally, also the operator $\widehat{A}$ emanates from $A$ defined in (2.32) in the sense that the operators

$$
\begin{aligned}
\left(\widehat{K}_{j, k}^{D} \widehat{\psi}_{j}\right)(t) & :=\int_{0}^{2 \pi} \frac{\partial \Phi\left(z_{k}(t), z_{j}(\tau)\right)}{\partial \nu\left(z_{j}(\tau)\right)} \widehat{\psi}_{j}(\tau) d \tau, \quad t \in[0,2 \pi], \\
\left(\widehat{K}_{j}^{D, \Gamma} \widehat{\psi}_{j}\right)(t) & :=\int_{0}^{2 \pi} \frac{\partial \Phi\left(\gamma(\cos t), z_{j}(\tau)\right)}{\partial \nu\left(z_{j}(\tau)\right)} \widehat{\psi}_{j}(\tau) d \tau, \quad t \in[0, \pi], \\
\left(\widehat{K}_{k}^{\Gamma, D} \widetilde{\varphi}\right)(t) & :=\left(\widetilde{K}_{k}^{\Gamma, D} \widetilde{\varphi}\right)\left(z_{k}(t)\right), \quad t \in[0,2 \pi]
\end{aligned}
$$

are obtained from the respective operators $K_{j, k}^{D}, \widetilde{K}_{j}^{D, \Gamma}$ and $\widetilde{K}_{k}^{\Gamma, D}$ that are defined in $(2.14),(2.29)$ and respectively $(2.26)$, by inserting the appropriate parametrizations $z_{j}$.

From (4.2) and from the form of the operators (4.4) - (4.6) we see that the collocation method is only semi-discrete as it is still required to evaluate the integral operator $\widehat{A}$ and the right-hand side $\widehat{f}$, which also is an integral operator, but with a fixed density. Nevertheless, the operator $\widehat{S}$, which apart from the identities on $C_{2 \pi}$ also contains the operator $\widetilde{L}$ defined in (2.22), can be evaluated exactly on $\widetilde{W}^{(m)}$ due to the following Lemma. 
Lemma 4.2. The operator $\widetilde{L}$ defined in (2.22) maps $T_{n, e}$ bijectively onto itself. It can be evaluated exactly on elements of $T_{n, e}$. In particular, let

$$
L_{j}^{n}(t):=\frac{1}{2 n}\left(1+2 \sum_{k=1}^{n-1} \cos \left(k\left(t-t_{j}\right)\right)+\cos \left(n\left(t-t_{j}\right)\right)\right), \quad t \in[0,2 \pi],
$$

be the Lagrange basis of $T_{n}$ for $j=0, \ldots, 2 n-1$. Then $\widetilde{L}$ takes the values

$$
\left(\widetilde{L} L_{j}^{n}\right)(t)=\frac{1}{2 n}\left(1+\sum_{k=1}^{n-1} \frac{1}{k} \cos \left(k\left(t-t_{j}\right)\right)+\frac{1}{2 n} \cos \left(n\left(t-t_{j}\right)\right)\right), \quad t \in[0,2 \pi] .
$$

Proof. see [36], Section 5.1.

Nevertheless, the operator $\widehat{A}$ and the right-hand side $\widehat{f}$ require further treatment to render a fully discrete approximation scheme. We will resort to a quadrature method, which in combination with trigonometric interpolation yields excellent convergence rates.

In particular, we carry on by discretizing the occuring integral operators using the composite trapezoidal rule with equidistantly spaced interpolation points given by the collocation points (4.1). This yields a fully discrete operator $A^{(m)}: \mathbb{R}^{M} \rightarrow \mathbb{R}^{M}$ and an approximation $f^{(m)} \in \mathbb{R}^{M}$ to the right-hand side $\widehat{f}$ that can be easily implemented. Substituting $A^{(m)}$ for $\widehat{A}$ and $f^{(m)}$ for $\widehat{f}$ in collocation method formulation (4.2), we arrive at a fully discrete approximation scheme, that is then used to recover approximations $\Psi^{(m)} \in \widetilde{W}^{(m)}$ to the solution $\Psi$ of the system of integral equations (2.36).

Remark 4.3. When implementing the above approximation scheme it is important to bear two things in mind.

1. According to Corollary 1.20 the operator $\widetilde{L}$ is invertible from $C_{2 \pi, e}^{0, \alpha}$ to $C_{2 \pi, e}^{1, \alpha}$ in the representation given in (1.40). This representation in particular involves an integral over $[0,2 \pi]$. Hence, the elements of the Lagrange basis from Lemma 4.2 have interpolation points in $[0,2 \pi]$. As has been stated by Mönch in [51] these can be interpreted as being taken from $[0, \pi]$ using the symmetry of $2 \pi$-periodic, even functions with respect to $\pi$. In fact, it is this observation, which eliminates linearly dependent equations from the discretized system of equations. With this approach the evaluation of $\widetilde{L}$ on elements of $T_{n, e}$ has to be done using the fact that the Lagrange basis of $T_{n, e}$ is given by $\left\{L_{0}^{n}, L_{n}^{n}, L_{j}^{n}+L_{2 n-j}^{n}: j=1, \ldots, n-1\right\}$. 
2. The operator $-S^{\Gamma}\left(\frac{I}{|\Gamma|}\right)$ that appears in the right-hand side (4.3) also contains a logarithmic singularity, which has to be treated adequately in discretizing. We propose to split the operator $-S^{\Gamma}\left(\frac{I}{|\Gamma|}\right)$ analogously to $(2.21)$ into a part containing the singularity and a part that has a continuous kernel, which will again lead to the operators $\widetilde{L}$ and $L_{0}$ as defined in (2.22) and (2.23). Then we use the fact that we can evaluate the singular integral exactly as the density is a constant.

So far, the presented scheme deals with obtaining the approximate solution $\Psi^{(m)}$ to (4.2). We note that this is crucial for approximating the potential $u$ that solves Problem 2.1 or any of its partial derivatives which correspond to components of the magnetic field $\mathbf{B}$ via (1). The argument for this is as follows. Once we have obtained $\Psi^{(m)}$ we can derive a semi-discrete approximation to $u$ from the representation (2.10) by discretizing the occuring boundary integrals in the same fashion as described above. As the occurring kernels are analytic for $x \in \mathbb{R}^{2} \backslash(\Gamma \cup \partial D)$, we can also derive approximations to the partial derivatives by differentiating the kernels accordingly. Furthermore, the analyticity of the integrands implies that we can expect the convergence rates of the potential and its derivatives to be as good as the convergence rates for $\Psi^{(m)}$.

In the following, we will accordingly present the error analysis for the approximation scheme described above, deriving exponential convergence rates for the approximate solutions $\Psi^{(m)}$ of the fully discrete system to the solution $\Psi$ of the exact system. To keep the error analysis as straight forward as possible, we interpret the approximation scheme as a projection method, which allows us to use standard arguments for projection methods for equations of the second kind as described in [47], Section 13.2. In a first step we present a result for the semi-discrete scheme.

Lemma 4.4. Given equation (2.36) with sufficiently smooth parametrizations of $\partial D$ as well as the finite-dimensional subspace $\widetilde{W}^{(m)}$ and the corresponding projection operator

$$
\widetilde{P}^{(m)}:=\left(\begin{array}{cccc}
P_{m_{0}, e}^{1, \alpha} & 0 & \cdots & 0 \\
0 & P_{m_{1}} & \ddots & \vdots \\
\vdots & \ddots & \ddots & 0 \\
0 & \cdots & 0 & P_{m_{n}}
\end{array}\right)
$$

where $P_{m_{0}}^{1, \alpha}: C_{2 \pi, e}^{1, \alpha} \rightarrow T_{m_{0}, e}$ and $P_{m_{i}}: C_{2 \pi} \rightarrow T_{m_{i}}$ for $m_{1}, \ldots, m_{n} \in \mathbb{N}$ are the interpolation operators corresponding to the collocation points (4.1), then the approximating equation

$$
\widetilde{P}^{(m)}(\widehat{S}+\widehat{A}) \Psi^{(m)}=\widetilde{P}^{(m)} f
$$


is uniquely solvable for sufficiently large $m_{0}, \ldots, m_{n}$, and we have the error estimate

$$
\left\|\Psi^{(m)}-\Psi\right\| \leq C \frac{\ln \tilde{m}}{\widetilde{m}^{\nu}}\|\Psi\|,
$$

where $\Psi$ is the solution to the exact equation (2.36).

Proof. From Theorem 2.10 we already know that the operator $S+A$ is injective and consists of an invertible operator $S$ with bounded inverse and a compact operator $A$. Consequently, the same holds also for the operator $\widehat{S}+\widehat{A}$ that is obtained by parametrization. Moreover, Lemma 4.2 shows that the operator $\widehat{S}$ is bijective from $\widetilde{W}^{(m)}$ to $\widetilde{W}^{(m)}$, so that for the semi-discrete projection method to converge (see [47], Theorem 13.12), we are left to prove

$$
\left\|\widetilde{P}^{(m)} \widehat{A}-\widehat{A}\right\| \rightarrow 0, \quad m \rightarrow \infty .
$$

Considering each entry of $\left(\widetilde{P}^{(m)} \widehat{A}-\widehat{A}\right)$ separately, we use the fact that the entries of $\widehat{A}$ are bounded operators together with the general estimate (see [59])

$$
\left\|P_{n} g-g\right\|_{l, \beta} \leq C \frac{\ln n}{n^{k-l+\beta-\alpha}}\|g\|_{k, \alpha}
$$

for the interpolation operator $P_{n}$ from $C_{2 \pi}^{l, \beta}$ to $T_{n}$ and $g \in C_{2 \pi}^{k, \alpha}, k, l \in \mathbb{N} \cup\{0\}$ with $l \leq k, 0<\beta \leq \alpha \leq 1$ and some constant $C$ depending on $l, k, \alpha$ and $\beta$. From this we see that, as the additional regularity of the parametrizations yields also additional regularity of the densities, we have convergence in (4.9) in the limit as $m=\left(m_{0}, \ldots, m_{n}\right)$ tends to infinity. This establishes unique solvability of the approximating equation (4.7) for sufficiently large $m_{0}, \ldots, m_{n}$. The error estimate (4.8) again follows from (4.10) and the general estimate

$$
\left\|\Psi^{(m)}-\Psi\right\| \leq M\left\|\widetilde{P}^{(m)} S \Psi-S \Psi\right\|
$$

(see [47], Theorem 13.12). In (4.8) we have set $\nu:=\min \left\{\beta-\alpha, \alpha_{1}, \ldots, \alpha_{n}\right\}$ and $\widetilde{m}:=\min \left\{m_{0}, \ldots, m_{n}\right\}$. Here, $\beta-\alpha$ is given by $(4.10)$, and $\alpha_{1}, \ldots, \alpha_{n}$ are given by similar estimates for the operators $P_{m_{i}}$ (see [47], Theorem 11.6).

Although Lemma 4.4 gives a positive result, it is still very poor with regard to the convergence rate (4.8). Nevertheless, we are able to improve the convergence rate by assuming higher regularity from the parametrizations $z_{i}, i=1, \ldots, n$, and $\gamma$. The key observations here is the fact that in the case of a real-valued, $2 \pi$-periodic and analytic function $g$ we have the estimate

$$
\left\|P_{n} g-g\right\|_{\infty} \leq C e^{-n s}
$$

for trigonometric interpolation, where the constants $C$ and $s$ solely depend on $g$ (see [45]). 
Corollary 4.5. Provided the parametrizations $z_{i}$ of $\partial D_{i}, i=1, \ldots, n$, and $\gamma$ of $\Gamma$ are analytic, the error estimate (4.8) can be sharpened to

$$
\left\|\Psi^{(m)}-\Psi\right\|_{\infty} \leq C e^{-\widetilde{m} s}
$$

where $\widetilde{m}:=\min \left\{m_{0}, \ldots, m_{n}\right\}$, and $C$ and $s$ are constants depending on the exact solution $\Psi$ of $(2.36)$.

Proof. Analogous to the proof of Lemma 4.4 using the error estimate (4.11) instead of (4.10).

In a second step we now use the results from above for analogous results in the case of the fully discrete scheme. Here it is again necessary to increase the assumptions on the regularity of the parametrizations $z_{i}, i=1, \ldots, n$ in order to secure that the kernels $\widehat{K}_{i, i}^{D}$ for $i=1, \ldots, n$, are twice continuously differentiable.

Lemma 4.6. Under the assumptions of Lemma 4.4 with increased assumptions on the regularity of the parametrizations $z_{i}, i=1, \ldots, n$, the approximating equation

$$
\widetilde{P}^{(m)}\left(S+A^{(m)}\right) \Psi^{(m)}=\widetilde{P}^{(m)} f^{(m)}
$$

of the fully discrete approximation scheme is uniquely solvable for sufficiently large $m_{0}, \ldots, m_{n}$, and we have the error estimate

$$
\left\|\Psi^{(m)}-\Psi\right\| \leq C \frac{\ln \widetilde{m}}{\widetilde{m}^{\nu}}
$$

Proof. According to the general result for fully discrete projection schemes given in [47], Theorem 13.13, to show unique solvability of the approximating equation of the fully discrete scheme, we need to establish pointwise convergence

$$
\left(P^{(m)} A^{(m)}-P^{(m)} \widehat{A}\right) \Psi \rightarrow 0
$$

for all $\Psi \in \widetilde{W}_{0}$ as $m \rightarrow \infty$, which follows from the pointwise convergence of the composite trapezoidal rule and the fact that the operator $P^{(m)}$ is bounded because of the additional regularity of the parametrizations. Furthermore, we need to show convergence

$$
\left\|P^{(m)} A^{(m)}-P^{(m)} \widehat{A}\right\|_{\widetilde{W}^{(m)} \rightarrow \widetilde{W}^{(m)}} \rightarrow 0
$$

in the operator norm on $\widetilde{W}^{(m)}$ as $m \rightarrow \infty$. But this again follows from the additional regularity since the composite trapezoidal rule yields quadratic convergence in the case of twice continuously differentiable functions. Thus, using standard 
arguments we can establish unique solvability of the approximating equation of the fully discrete scheme. Using the general estimate

$$
\begin{gathered}
\left\|\Psi^{(m)}-\Psi\right\| \leq M\left\{\left\|P^{(m)} S \Psi-S \Psi\right\|+\left\|P^{(m)}\left(A^{(m)}-\widehat{A}\right) \Psi\right\|\right. \\
\left.+\left\|P^{(m)}\left(f^{(m)}-f\right)\right\|\right\}
\end{gathered}
$$

(see [47], Theorem 13.13), we compute the error estimate (4.12) from the corresponding result (4.8) for the semi-discrete case, from the general estimate (4.10) and from the quadratic convergence of the composite trapezoidal rule. In (4.12), we have set $\nu:=\min \left\{\beta-\alpha, \alpha_{1}, \ldots, \alpha_{n}\right\}$ and $\widetilde{m}:=\min \left\{m_{0}, \ldots, m_{n}\right\}$ as in the semi-discrete case. Here again, $\beta-\alpha$ is given by (4.10), and $\alpha_{1}, \ldots, \alpha_{n}$ are given by similar estimates for the operators $P_{m_{i}}$ (see [47], Theorem 11.6).

Again the result of Lemma 4.6 is not satisfying with regard to the convergence rate (4.12). But as in the semi-discrete case, we are able to increase the convergence rate provided we assume a higher regularity of the parametrizations.

Corollary 4.7. Provided the parametrizations $z_{i}$ of $\partial D_{i}, i=1, \ldots, n$, and $\gamma$ of $\Gamma$ are analytic, the error estimate (4.12) can be sharpened to

$$
\left\|\Psi^{(m)}-\Psi\right\|_{\infty} \leq C e^{-\widetilde{m} s}
$$

where $\widetilde{m}:=\min \left\{m_{0}, \ldots, m_{n}\right\}$, and $C$ and $s$ are constants depending on the exact solution $\Psi$ of $(2.36)$.

Proof. Analogous to the proof of Lemma 4.6 using the error estimate (4.11) for the trigonometric interpolation of analytic functions.

\subsection{A steepest descent algorithm for the Geo- metric Optimization Problem}

In the following we will proceed in turning the sensitivity results of Section 3.5 into a numerical approximation scheme.

One of the major difficulties in geometric optimization is that the set of admissible domains with respect to which the optimization should be performed does not have a linear structure. Hence, the usual optimization approaches are prone to fail if employed naively.

In Section 3.4 we have already seen an approach how to describe the set of admissible domains in an affine setting. There, we have made use of compactly 
supported, $C^{2}$-smooth vector fields as a diffeomorphic perturbation of the identity. Unfortunately, this description of the set of admissible domains is rather unhandy for setting up an approximation scheme.

For the rest of this section we will assume that the domain $D$ is bounded, simply connected and starlike with a smooth boundary curve. In particular, let

$$
V_{a d}:=\left\{r \in C_{2 \pi}^{2}: r>0\right\} \subset C_{2 \pi}^{2}
$$

and define for $r \in V_{a d}$ and $x_{0} \in \mathbb{R}^{2}$

$$
D_{x_{0}, r}:=\left\{x \in \mathbb{R}^{2}: x=x_{0}+\operatorname{sr}(t)\left(\begin{array}{c}
\cos t \\
\sin t
\end{array}\right), s \in[0,1), t \in[0,2 \pi)\right\} .
$$

Then the boundary of $D_{x_{0}, r}$ is given by

$$
\partial D_{x_{0}, r}:=\left\{x \in \mathbb{R}^{2}: x=x_{0}+r(t)\left(\begin{array}{c}
\cos t \\
\sin t
\end{array}\right), t \in[0,2 \pi)\right\} .
$$

From the definition of $D_{x_{0}, r}$ it is immediately clear that the boundaries are admissible provided $\Gamma \cap D_{x_{0}, r}=\emptyset$. In order to use the class of domains given by $V_{a d}$ for the Geometric Optimization Problem we need the sensitivity analysis from Chapter 3 to be applicable for boundaries that are induced by $V_{a d}$. To this end we relate $V_{a d}$ to the space $U^{m, \eta}\left(D_{0}\right)$ of compactly supported vector fields as introduced in Definition 3.26. The following theorem states that the set of admissible domains that is induced by $V_{a d}$ can be described in terms of diffeomorphisms.

Theorem 4.8. Let $x_{0} \in \mathbb{R}^{2}$, and let $r_{1}, r_{2} \in V_{a d}$ be the radial functions for two domains $D_{x_{0}, r_{1}}$ and $D_{x_{0}, r_{2}}$. Then there exists a compactly supported, twice continuously mapping $f$ such that $(I d-f)$ is a $C^{2}$-diffeomorphism on $\mathbb{R}^{2}$ with the property that $(I d-f)\left(D_{x_{0}, r_{1}}\right)=D_{x_{0}, r_{2}}$.

Proof. The proof constructs $f$ in three steps. Without loss of generality we assume that $x_{0}=0$. Furthermore we consider $\mathbb{R}^{2}$ using polar coordinates.

In a first step we consider the mapping $g: \mathbb{R}^{2} \rightarrow \mathbb{R}^{2}$ given by

$$
g(t, r):=\left(t, \frac{r_{2}(t)}{r_{1}(t)} r\right), \quad t \in[0,2 \pi), r \in[0, \infty)
$$

We immediately observe that $g$ is twice continuously differentiable, and has the property that $g\left(D_{x_{0}, r_{1}}\right)=D_{x_{0}, r_{2}}$. Furthermore, $g$ is invertible due to the positivity of $r_{1}$, with the inverse of $g$ being given by

$$
g^{-1}(t, r):=\left(t, \frac{r_{1}(t)}{r_{2}(t)} r\right), \quad t \in[0,2 \pi), r \in[0, \infty)
$$


which is again well-defined as $r_{2}$ is positive. Hence, we have constructed a $C^{2}$ diffeomorphism that maps $D_{x_{0}, r_{1}}$ bijectively onto $D_{x_{0}, r_{2}}$.

In a second step we introduce $\widetilde{f}: \mathbb{R}^{2} \rightarrow \mathbb{R}^{2}$ by setting

$$
\widetilde{f}(t, r):=\left(t, \frac{r}{r_{1}(t)}\left(r_{1}(t)-r_{2}(t)\right)\right), \quad t \in[0,2 \pi), r \in[0, \infty),
$$

and observe that $\tilde{f}$ satisfies Id $-\tilde{f}=g$. Hence Id $-\tilde{f}$ is a $C^{2}$-diffeomorphism and a perturbation of the identity.

In the last step it remains to modify $\tilde{f}$ using an appropriate $C^{2}$-smooth cut-off function $\alpha: \mathbb{R}^{2} \rightarrow \mathbb{R}^{2}$ that has support in a neighbourhood of $\partial D_{x_{0}, r_{1}}$. Then the support of $f:=\alpha \tilde{f}$ is compact.

The result of Theorem 4.8 is already very promising as it has shown how we can think of the space $V_{a d}$ as being obtained locally by perturbations of a fixed reference domain $D_{0}$ in the sense of Definition 3.26. Still, for the sensitivity analysis of Chapter 3 to be applicable, $V_{a d}$ has to satisfy some additional properties which we are going to verify in the following lemma.

Lemma 4.9. The set $V_{a d}$ as defined in (4.13) is open and convex with respect to the $C^{2}$-norm.

Proof. To see that $V_{a d}$ is open, we let $r \in V_{a d}$ and set

$$
\varepsilon:=\inf _{t \in \mathbb{R}} r(t)=\min _{t \in[0,2 \pi]} r(t)
$$

using the periodicity of $r$. Thus, we obtain that $\varepsilon>0$ due to the positivity of $r$. Then for any $v \in C_{2 \pi}^{2}$ satisfying $\|v-r\|_{C^{2}}<\frac{\varepsilon}{2}$ we calculate

$$
|v(t)-r(t)| \leq\|v-r\|_{\infty} \leq\|v-r\|_{C^{2}}<\frac{\varepsilon}{2}
$$

for any $t \in \mathbb{R}$. Now the positivity of $r$ yields in particular that

$$
v(t)>r(t)-\frac{\varepsilon}{2} \geq \frac{\varepsilon}{2}>0
$$

for any $t \in \mathbb{R}$. Hence, $v \in V_{a d}$ which settles openness of $V_{a d}$.

Convexity follows directly from the definition $V_{a d}$ as $C_{2 \pi}^{2}$ is a linear space and we have for $r_{1}, r_{2} \in V_{a d}$ the pointwise estimate

$$
\lambda r_{1}(t)+(1-\lambda) r_{2}(t)>\min \left\{r_{1}(t), r_{2}(t)\right\}>0
$$

for any $\lambda \in[0,1]$ and any $t \in \mathbb{R}$. 
By virtue of Theorem 4.8 and Lemma 4.9 we can now apply the sensitvity analysis also to the restriction of $\mathcal{U}_{a d}$ to those admissible boundaries that are given in terms of $V_{a d}$ for a set of finitely many given center points $C_{D}:=\left\{x_{0}^{(1)}, \ldots, x_{0}^{(n)}\right\}$. In other words, we may consider Problem 3.3 also for the set of admissible boundaries $\mathcal{U}_{a d}^{\prime} \subset \mathcal{U}_{a d}$, where

$$
\mathcal{U}_{a d}^{\prime}:=\left\{\partial D_{x_{0}, r} \subset \mathbb{R}^{2}: r \in V_{a d}, x_{0} \in C_{D}, \bar{D}_{x_{0}, r} \cap \Gamma=\emptyset\right\} .
$$

We note that also the existence result of Theorem 3.37 remains valid for $\mathcal{U}_{\text {ad }}^{\prime}$.

For implementation purposes $V_{a d}$ is much more easily accessible than $U^{m, \eta}\left(D_{0}\right)$, but it still remains infeasible in its infinite-dimensional generality. Only the restriction to a finite-dimensional subspace of $C_{2 \pi}^{2}$ will make the algorithmic idea practicable. The choice of a finite-dimensional subspace (or a collection subspaces of this kind) is very delicate. On the one hand it should incorporate the flexibility of the original space $C_{2 \pi}^{2}$, where we consider the basis functions as being taken from. Thus, it is desirable that the finite-dimensional subspace satisfies a kind of denseness property. On the other hand it should be appropriate to the problem, and can hence be regarded as one of the most important issues in the design of an approximation scheme.

In the following we will consider two choices for collections of finite-dimensional subspaces. The first is the $(2 n+1)$-dimensional space of trigonometric polynomials of order $\leq n$ given by

$$
T_{n}:=\operatorname{span}(\{\cos k t: k=0, \ldots, n\} \cup\{\sin k t: k=1, \ldots, n\}) .
$$

Trigonometric polynomials are the most simple and straightforward approximation space to be considered. The denseness property holds due to the Weierstraß Theorem, they are versatile and simple to implement.

Nevertheless, they also display some features which can be interpreted as drawbacks. So, the basis functions have non-local support, they are highly oscillatory for large $n$, and the basis functions take both positive and negative values. This makes additional considerations necessary to ensure that $r \in T_{n}$ satisfies $r>0$ when $T_{n}$ is used as a subspace of $V_{a d}$.

The second collection of finite-dimensional subspaces we are going to consider in the numerical examples is given by

$$
\widetilde{T}_{n, k}:=\operatorname{span}\left(\{1\} \cup\left\{h_{j}\left(\cdot-t_{i}\right): j=1, \ldots, n, i=0, \ldots, k\right\}\right)
$$

for $t_{i}=\frac{2 \pi i}{k}, i=0, \ldots, k$, where the basis functions $h_{j}$ are given by the periodic extensions of

$$
\widetilde{h}_{j}(t):=\pi^{-2(j+1)} \sin ^{2}(j t)\left(\pi^{2}-t^{2}\right)^{j+1}, \quad j \in \mathbb{N}, t \in[-\pi, \pi) .
$$


A direct calculation shows that $\widetilde{h}_{j}$ can be extended periodically as a twice continuously differentiable function on $\mathbb{R}$ for any $j \in \mathbb{N}$. This is due to the order of the zero at $t= \pm \pi$ that comes from the damping factor. Furthermore, we directly observe that $\widetilde{h}_{j}$ is non-negative, and has a localized support. The behaviour of the basis functions $\widetilde{h}_{j}$ is illustrated in Figure 4.1 .

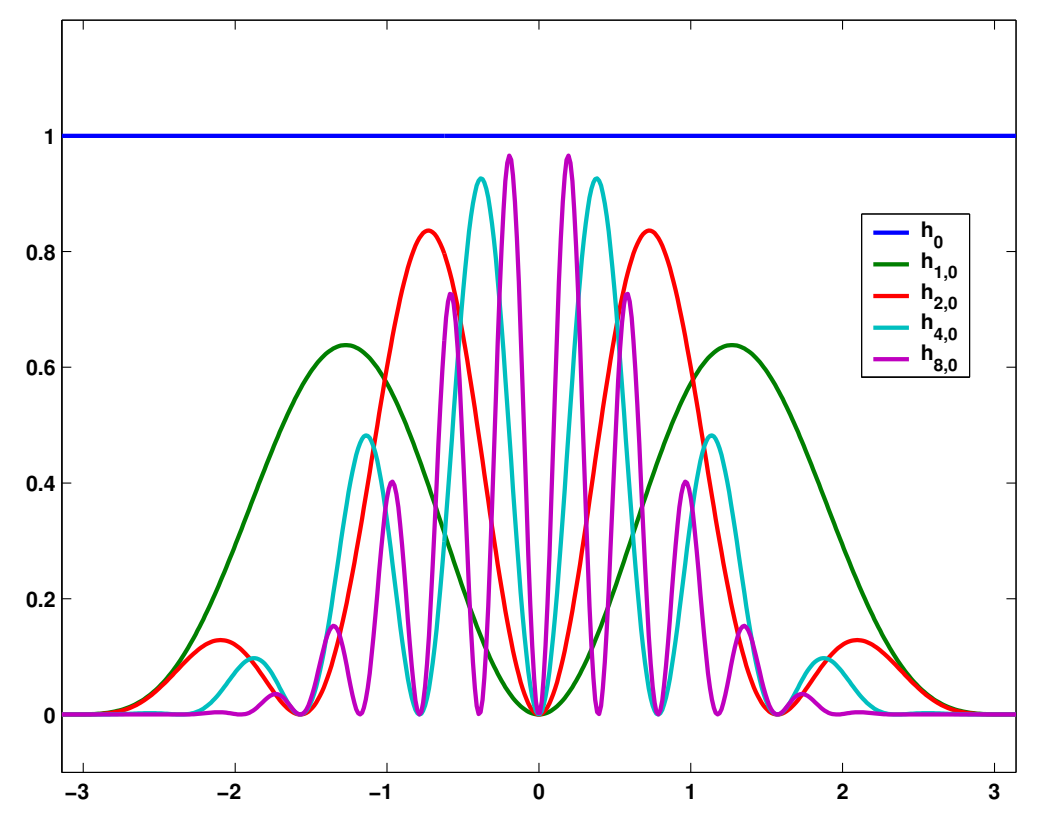

Figure 4.1: Illustration of the basis functions $h_{j}$ given in (4.19) for $j=1,2,4,8$ together with the constant basis function.

The choice for $\widetilde{T}_{n, k}$ comes from the perspective of design. The examples of Section 5.2 that have been performed with trigonometric polynomials display that the effects of the functional $F$ are rather localized even for this type of basis functions that have a non-local support. The results are spoiled by oscillations in the boundary curve in regions that are rather far away from the arc. Contrarily, the space $\widetilde{T}_{n, k}$ contains functions with a more localized support and should consequently not display these phenomena. Moreover, the positivity of the basis functions should help the algorithm in not getting trapped in local boundary minima that are due to the radial function becoming negative in 'unimportant' regions of the boundary.

With these finite-dimensional approximation spaces for $V_{a d}$ in hand, the Geometric Optimization Problem 3.3 now becomes tractable. It reduces to a constrained optimization problem for the coefficients of the basis functions of $T_{n}$ or $\widetilde{T}_{n, k}$ respectively. In this sense, the set of admissible boundaries that are induced by $T_{n}$ can be characterized by

$$
P_{n}:=\left\{a \in \mathbb{R}^{2 n+1}: r(a) \in V_{a d} \cap T_{n}, \partial D_{x_{0}, r(a)} \in \mathcal{U}_{a d}^{\prime}\right\},
$$


where the radial function $r(a)$ is defined by

$$
(r(a))(t):=a_{0}+\sum_{k=1}^{n} a_{2 k-1} \sin k t+a_{2 k} \cos k t, \quad t \in \mathbb{R} .
$$

Similarly, the set of admissible boundaries that are induced by $\widetilde{T}_{n, k}$ can be characterized via

$$
\widetilde{P}_{n, k}:=\left\{a \in \mathbb{R}^{n(k+1)+1}: r(a) \in V_{a d} \cap \widetilde{T}_{n, k}, \partial D_{x_{0}, r(a)} \in \mathcal{U}_{a d}^{\prime}\right\}
$$

where the radial function $r(a)$ is given through

$$
(r(a))(t):=a_{0}+\sum_{i=0}^{k} \sum_{j=1}^{n} a_{i j} h_{j}\left(t-t_{i}\right), \quad t \in \mathbb{R} .
$$

The numerical approximation scheme which we are going to present now is effectively solving a finite-dimensional analogue to Problem 3.3 given by

$$
\min _{a \in P} F\left(\partial D_{x_{0}, r(a)}\right)+\beta G_{c}\left(\partial D_{x_{0}, r(a)}\right)+\mu G_{\vartheta, d}\left(\partial D_{x_{0}, r(a)}\right),
$$

where $P$ is either given by $P_{n}$ as defined in (4.20) or by $\widetilde{P}_{n, k}$ as defined in (4.21). The additional terms $G_{\vartheta, d}$ and $G_{c}$ are introduced to ensure that the algorithm runs stably and incorporates the admissibility constraint $D \cap \Gamma=\emptyset$. We will discuss each term in more detail after considering the feasibility of the overall algorithm.

We suggest a simple Steepest Descent Algorithm to solve the minimization problem (4.22). In particular, we know from Theorem 3.36 and Theorem 4.8 that the functional $F$ is differentiable on $\mathcal{U}_{a d}^{\prime}$. (The differentiability of the terms $G_{\vartheta, d}$ and $G_{c}$ will be settled later in this section.) So we have for $a \in P$ and $h \in T$ with $\|h\|_{C^{2}}$ sufficiently small that

$$
\begin{aligned}
\left(F+\beta G_{c}+\right. & \left.\mu G_{\vartheta, d}\right)(r(a)+h) \\
= & \left(F+\beta G_{c}+\mu G_{\vartheta, d}\right)(r(a)) \\
& \quad+F^{\prime}[r(a) ; h]+\beta G_{c}^{\prime}[r(a) ; h]+\mu G_{\vartheta, d}^{\prime}[r(a) ; h]+o(\|h\|) .
\end{aligned}
$$

Here, we use the radial functions $r(a)+h$ as a short-hand notation for $\partial D_{x_{0}, r(a)+h}$, and $r(a)$ as an abbreviation for $\partial D_{x_{0}, r(a)}$ respectively. Note that the perturbation $h \in T$ is identified with the perturbation it induces in the sense of Theorem 4.8, and $T$ is given either by $T_{n}$ or by $\widetilde{T}_{n, k}$ depending on which approximation space we are considering. Then the steepest descent algorithm reads 


$$
\begin{aligned}
a^{(n+1)}:= & a^{(n)}-\alpha_{n}\left[\left(F^{\prime}\left[r\left(a^{(n)}\right) ; h_{1}\right], \ldots, F^{\prime}\left[r\left(a^{(n)}\right) ; h_{N}\right]\right)^{T}\right. \\
& +\beta\left(G_{c}^{\prime}\left[r\left(a^{(n)}\right) ; h_{1}\right], \ldots, G_{c}^{\prime}\left[r\left(a^{(n)}\right) ; h_{N}\right]\right)^{T} \\
& \left.+\mu_{n}\left(G_{\vartheta, d}^{\prime}\left[r\left(a^{(n)}\right) ; h_{1}\right], \ldots, G_{\vartheta, d}^{\prime}\left[r\left(a^{(n)}\right) ; h_{N}\right]\right)^{T}\right],
\end{aligned}
$$

where $h_{1}, \ldots, h_{N}$ are the basis functions of $T, \alpha_{n}>0$ is a sufficiently small step size parameter, and $\beta$ and $\mu_{n}$ are appropriately chosen regularization parameters.

Lemma 4.10. Let $a^{(0)} \in P$ and define $a^{(n)}$ recursively via (4.24) for $\alpha_{n} \in \mathbb{R}^{+}$ sufficiently small. Then the sequence

$$
\left(\left(F+\beta G_{c}+\mu G_{\vartheta, d}\right)\left(\partial D_{x_{0}, r\left(a^{(n)}\right)}\right)\right)_{n \in \mathbb{N}_{0}} \subset \mathbb{R}
$$

is monotonically decreasing.

Proof. The vector

$$
\left(F^{\prime}\left[r\left(a^{(n)}\right) ; h_{1}\right], \ldots, F^{\prime}\left[r\left(a^{(n)}\right) ; h_{N}\right]\right)^{T}
$$

has a natural interpretation in $C_{2 \pi}^{2}$ via

$$
\sum_{k=1}^{N} F^{\prime}\left[r\left(a^{(n)}\right) ; h_{k}\right] h_{k}
$$

and the analogue holds also for the other summands in (4.24). Hence, the updated iterate $a^{(n+1)}$ can be written in terms of the asymptotic expansion (4.23). Using the linearity of the Fréchet derivative, we can expand the corresponding expression in terms of the basis functions to obtain

$$
\begin{aligned}
(F+ & \left.\beta G_{c}+\mu G_{\vartheta, d}\right)\left(r\left(a^{(n+1)}\right)\right)-\left(F+\beta G_{c}+\mu G_{\vartheta, d}\right)\left(r\left(a^{(n)}\right)\right) \\
= & -\alpha_{n} \sum_{k=1}^{N}\left(F^{\prime}\left[r\left(a^{(n)}\right) ; h_{k}\right]^{2}+\beta^{2} G_{c}^{\prime}\left[r\left(a^{(n)}\right) ; h_{k}\right]^{2}+\mu^{2} G_{\vartheta, d}^{\prime}\left[r\left(a^{(n)}\right) ; h_{k}\right]^{2}\right) \\
& +o(\|h\|) .
\end{aligned}
$$

Now the statement follows from $\alpha_{n}$ and the sum being non-negative, and the faster decay of $o(\|h\|)$ by choosing $\alpha_{n}$ sufficiently small . 
Although this algorithm is analytically sound, it suffers from a severe drawback. The scaling parameters $\alpha_{n}$ are analytic quantities that are a priori not explicitly available. Hence, we suggest modifying the algorithm as to incorporating a line search procedure.

Algorithm 4.11 (Steepest Descent Algorithm with Line Search).

We a priori choose a regularization parameter $\beta>0$, and a gradient tolerance $\varepsilon_{g}>0$ below which we assume the gradient of $F+\beta G_{c}+\mu G_{\vartheta, d}$ to be vanishing.

For the line search we choose a set $\mathcal{A}$ of line search parameters and a step size tolerance $\varepsilon_{b}$ below which we assume a step to be too near to the boundary of $P$. Then the algorithm reads

- Set $n:=0$ and choose $a^{(0)} \in P$.

- Calculate

$$
\begin{aligned}
g_{f} & :=\left(F^{\prime}\left[r\left(a^{(n)}\right) ; h_{1}\right], \ldots, F^{\prime}\left[r\left(a^{(n)}\right) ; h_{N}\right]\right), \\
g_{c} & :=\left(G_{c}^{\prime}\left[r\left(a^{(n)}\right) ; h_{1}\right], \ldots, G_{c}^{\prime}\left[r\left(a^{(n)}\right) ; h_{N}\right]\right), \\
g_{\vartheta, d} & :=\left(G_{\vartheta, d}^{\prime}\left[r\left(a^{(n)}\right) ; h_{1}\right], \ldots, G_{\vartheta, d}^{\prime}\left[r\left(a^{(n)}\right) ; h_{N}\right]\right) .
\end{aligned}
$$

- Compute $\mu_{n}$ according to (4.30).

- If $\left\|g_{f}+\beta g_{c}+\mu_{n} g_{\vartheta, d}\right\| \leq \varepsilon_{g}: \quad S T O P$

$\Rightarrow \partial D_{x_{0}, r\left(a^{(n)}\right)}$ is a local minimizer.

Else

- Compute $\widetilde{a}_{i}:=a^{(n)}-\sigma_{i}\left(g_{f}+\beta g_{c}+\mu_{n} g_{\vartheta, d}\right)^{T}$ for all $\sigma_{i} \in \mathcal{A}$.

- If $\widetilde{a}_{i} \notin P:$ Set $f_{i}:=\infty$.

Else: Set $f_{i}:=F\left(\partial D_{x_{0}, r\left(\widetilde{a}_{i}\right)}\right)$

- Set $\alpha_{n}:=\sigma_{i^{*}}$ for $f_{i^{*}}=\min _{i} f_{i}$

- If $\alpha_{n} \leq \varepsilon_{b}: \quad S T O P$

$\Rightarrow \partial D_{x_{0}, r\left(a^{(n)}\right)}$ is a boundary point of $P$ locally minimizing $F$.

Else: Set $a^{(n+1)}:=a^{(n)}-\alpha_{n}\left(g_{f}+\beta g_{c}+\mu_{n} g_{\vartheta, d}\right)^{T}, n:=n+1$

and GOTO step 2.

Remark 4.12. We would like to emphasize at this point that the use of a line search procedure has both its advantages and drawbacks, especially if it is used in such a crude form as in Algorithm 4.11. On the one hand it makes the descent 
algorithm extremely efficient, since it enables to select the most favourable step size for one descent step. Moreover, it enables to detect inadmissible geometries on the fly.

On the other hand the line search procedure renders the algorithm rather inefficient in terms of computation time since for every step size $\sigma_{i} \in \mathcal{A}$ the resulting boundary parametrization has to be tested for admissibility. Furthermore, and computationally even more costly, then the Boundary Value Problem 2.1 has to be solved for each admissible $r\left(\widetilde{a}_{i}\right)$ in order to calculate $f_{i}$ and determine the most favourable step size for the current descent step.

In the remainder of this section we are going to discuss how the admissibility constraints $r(a)>0$ and $D \cap \Gamma=\emptyset$ are incorporated into the algorithm, and how we guarantee essentially non-oscillatory radial functions for $\partial D$.

Let us address the positivity of the radial function $r(a)$ as a first issue. As can be seen from Algorithm 4.11 the restriction $r>0$ is tested explicitly whenever an update for $\partial D_{x_{0}, r}$ is computed, by checking the sign of $r$ at all boundary points of the discrete approximation to $\partial D_{x_{0}, r}$. In the case $r<0$ for some point on the boundary, we reduce the step size parameter until the restriction $r>0$ is again satisfied at all boundary points. In this sense we also make use of the line search procedure described above.

Secondly, the algorithm has to ensure that $D_{x_{0}, r\left(\widetilde{a}_{i}\right)} \cap \Gamma=\emptyset$, a restriction which is rather delicate to ensure. The treatment of a related problem in theoretical physics (see [30]), where the magnetic environment consisted of half-spaces instead of bounded domains, has indicated that decreasing the distance between the magnets $D$ and the superconducting film $\Gamma$ also reduces the concentration of the current distribution in a neighbourhood of the endpoints of $\Gamma$. This behaviour is confirmed from our numerical experience. We have found in all numerical experiments that a decrease in the distance between $\Gamma$ and $D$ also caused a dominant decrease of $F$.

Consequently, the approximation scheme has to be built such that it prevents the domain $D$ and the arc $\Gamma$ from overlapping both theoretically and numerically. Hence, we suggest an Augmented Lagrangian Approach to overcome this difficulty for some specially designed geometric setups, which we consider the most important from a geometric and applicational point of view.

Suppose $\Gamma=[-1,1] \times\{0\} \subset \mathbb{R}^{2}$, and $x_{0} \in \mathbb{R}^{2}$ satisfies $\left\langle\frac{z_{-1}-x_{0}}{\left|z_{-1}-x_{0}\right|}, e_{1}\right\rangle=\cos \vartheta$ for an angle $|\vartheta|<c$, where $e_{1}$ is the first standard unit vector in $\mathbb{R}^{2}$ and $c$ is some appropriate constant, i.e. we have a situation as illustrated in Figure 4.2.

For this geometric setup we impose the following equality constraint

$$
G_{\vartheta, d}\left(\partial D_{x_{0}, r}\right)=0
$$

where

$$
G_{\vartheta, d}\left(\partial D_{x_{0}, r}\right):=\left\|z_{-1}-\left(x_{0}+r(\vartheta)\left(\begin{array}{c}
\cos \vartheta \\
\sin \vartheta
\end{array}\right)\right)\right\|-d
$$




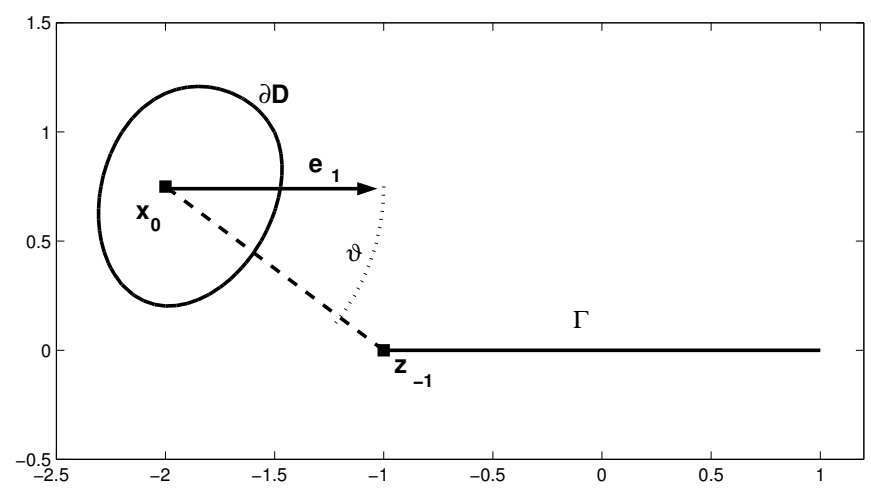

Figure 4.2: Schematic of positioning for $\Gamma$ and $x_{0}$

We note that by construction

$$
z_{-1}-x_{0}=\left|z_{-1}-x_{0}\right|\left(\begin{array}{c}
\cos \vartheta \\
\sin \vartheta
\end{array}\right)
$$

Hence $G_{\vartheta, d}\left(\partial D_{x_{0}, r}\right)=\left|z_{-1}-x_{0}\right|-(r(\vartheta)+d)$. Observing that $r(\vartheta)$ is linear with respect to the basis functions of $T_{n}$, or $\widetilde{T}_{n, k}$ respectively, we also have affine linearity of $G_{\vartheta, d}$. Consequently, with the help of Lemma 3.11, we see that $G_{\vartheta, d}$ is Fréchet differentiable with respect to $\partial D_{x_{0}, r} \in \mathcal{U}_{a d}^{\prime}$, and its Fréchet derivative is given by

$$
G^{\prime}[r(a) ; \cdot]=\left(G_{\vartheta, d}\left(h_{1}\right)+d-\left|z_{-1}-x_{0}\right|, \ldots, G_{\vartheta, d}\left(h_{N}\right)+d-\left|z_{-1}-x_{0}\right|\right),
$$

where $h_{k}$ are the basis functions of $T_{n}$, or $\widetilde{T}_{n, k}$ respectively. Hence, considering the so-called Augmented Lagrangian Functional

$$
\mathcal{L}_{\vartheta, d}\left(\partial D_{x_{0}, r}, \mu\right):=F\left(\partial D_{x_{0}, r}\right)+\beta G_{c}\left(\partial D_{x_{0}, r}\right)+\mu G_{\vartheta, d}\left(\partial D_{x_{0}, r}\right)
$$

leads to the necessary optimality conditions

$$
\begin{gathered}
F^{\prime}[r ; \cdot]+\beta G_{c}^{\prime}[r ; \cdot]+\mu G_{\vartheta, d}^{\prime}[r ; \cdot]=0, \\
G_{\vartheta, d}\left(\partial D_{x_{0}, r}\right)=0,
\end{gathered}
$$

which can be exploited in the context of the Steepest Descent Algorithm 4.11 such that each iterate satisfies $D \cap \Gamma=\emptyset$. Hence, we consider $\mathcal{L}_{\vartheta, d}(\cdot, \mu)$ and an element $r\left(a^{(n)}\right)$ satisfying (4.29). Then we find that by setting

$$
\mu_{n}:=-\frac{G_{\vartheta, d}\left(F^{\prime}\left[r\left(a^{(n)}\right) ; \cdot\right]+\beta G_{c}^{\prime}\left[r\left(a^{(n)}\right]\right)\right.}{G_{\vartheta, d}\left(G_{\vartheta, d}^{\prime}\left[r\left(a^{(n)}\right) ; \cdot\right]\right)}
$$

the new iterate

$$
a^{(n+1)}:=a^{(n)}-\alpha_{n}\left[F^{\prime}\left[r\left(a^{(n)}\right) ; \cdot\right]+\beta G_{c}^{\prime}\left[r\left(a^{(n)}\right) ; \cdot\right]+\mu_{n} G_{\vartheta, d}^{\prime}\left[r\left(a^{(n)}\right) ; \cdot\right]\right]
$$


also satisfies (4.29) due to the affine linearity of $G_{\vartheta, d}$ with respect to the basis functions. In this sense the Augmented Lagrangian Approach is superior to a naive Steepest Descent Algorithm as it automatically ensures $D \cap \Gamma=\emptyset$ for geometric situations as depicted in Figure 4.2.

Remark 4.13. In the case of less restrictive assumptions on the position of the centre $x_{0}$ of $D_{x_{0}, r}$ the Augmented Lagrangian Approach does not ensure the condition $D_{x_{0}, r} \cap \Gamma=\emptyset$ as easily. In such situations it might be better to consider modifications of $F$ that are obtained by penalization, which is beyond the scope of this thesis. A possible penalty term ensuring that $D_{x_{0}, r}$ and $\Gamma$ remain separated may be given for example by

$$
\int_{\partial D_{x_{0}, r}} \int_{\Gamma}\left|\ln \frac{\|x-y\|}{d}\right|^{p} d s(y) d s(x),
$$

where the parameter $d$ determines a favourable distance between $D_{x_{0}, r}$ and $\Gamma$, and the parameter $p$ incorporates a measure of the severeness of a deviation from that distance.

Nevertheless, the effect of such penalty terms in the behaviour of the Geometric Optimization Problem 3.3 remains somewhat unclear, since they penalize deviations from the favourable distance $d$ uniformly over $D_{x_{0}, r}$ and $\Gamma$. In particular, the effect of employing such a penalty term might lead to unnatural deformation and shrinking of $D_{x_{0}, r}$ due to the fact that the penalty term also seeks to decrease the distance between the side of $D_{x_{0}, r}$ that does not face $\Gamma$ and the endpoint of $\Gamma$ that does not face $D_{x_{0}, r}$.

Finally, we discuss the suppression of possible oscillations in the radial function. The need for this is justified from the construction of $T_{n}$ and $\widetilde{T}_{n, k}$. Both spaces contain rapidly oscillating basis functions when the dimension of the approximation space is chosen large. This may cause an oscillatory behaviour of the radial function $r$, which is supported from our numerical experience. According examples will be presented in Section 5.2.

The algorithm should therefore be able to suppress oscillatory behaviour of $r$ appropriately. This task is also rather delicate since Genenko et al. propose in [30] that regions with high curvature are a desired feature for the problem. In [30] Genenko et al. predict an improvement in the current distribution for a superconducting film in the vicinity of a magnetic environment that is obtained from deforming a half-space via conformal mappings. In particular it was found that current distribution was less inhomogeneous when the boundary of the magnetic environment was given by

$$
\partial D_{1}=\left\{x=\left(x_{1}, x_{2}\right) \in \mathbb{R}^{2}: x_{1}+i x_{2}=2\left(1+i \frac{w_{2}}{2}\right)^{\frac{1}{2}}, w_{2} \in \mathbb{R}\right\},
$$

where the constant 2 is due to the length of $\Gamma$ and where the square root is interpreted by choosing the main branch of the logarithm, instead of the undeformed 
half-space boundary $\left\{x=\left(x_{1}, x_{2}\right) \in \mathbb{R}^{2}: x_{1}=2\right\}$. The situation is depicted in Figure 4.3. It shows that regions of high curvature can be expected in the vicinity of the endpoints of $\Gamma$, whereas we judge them as unnatural in parts of the boundary $\partial D$ that are not close to the endpoints. The numerical experiments from Section 5.2 support this point of view.

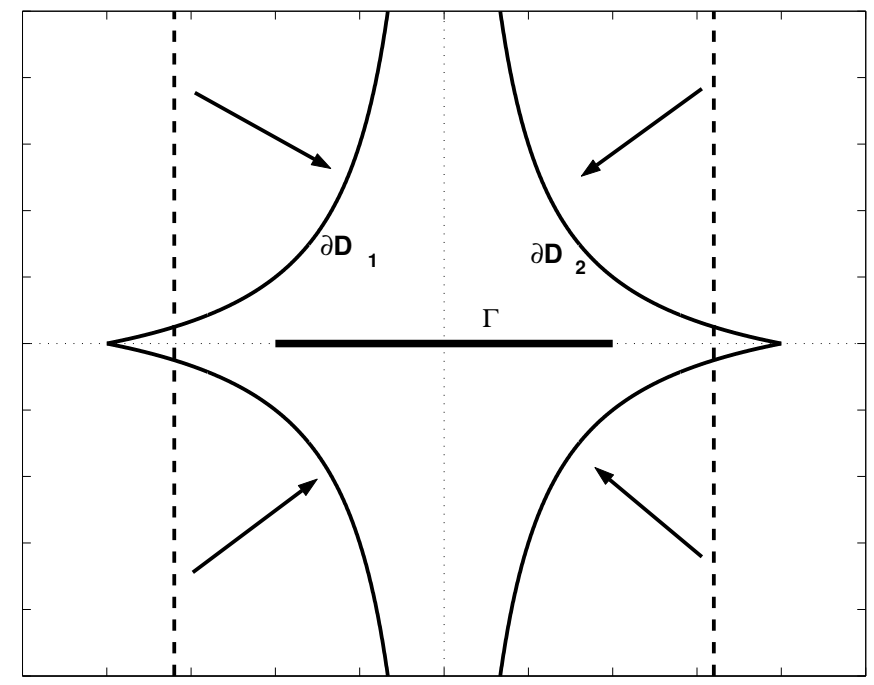

Figure 4.3: Example for the homogenizating effect on the current distribution by conformal mapping of two half-spaces.

The chosen functional (3.4) provides no inherent means of damping such oscillatory behaviour. Hence, we suggest regularizing the Steepest Descent Algorithm with respect to damping the formation of regions with high curvature in the boundary parametrization for $\partial D_{x_{0}, r}$. In accomplishing this we follow a rather standard procedure by penalizing the second derivative of the boundary parametrization, i.e. we add the penalty term

$$
\begin{aligned}
G_{c}\left(\partial D_{x_{0}, r}\right) & :=\int_{0}^{2 \pi}\left\|\left(r(t)\left(\begin{array}{c}
\cos t \\
\sin t
\end{array}\right)\right)^{\prime \prime}\right\|^{2} d t \\
& =\int_{0}^{2 \pi}\left(r^{\prime \prime}(t)-r(t)\right)^{2}+2 r^{\prime}(t)^{2} d t
\end{aligned}
$$

which will be weighted by some regularization parameter $\beta$.

The Steepest Descent Algorithm 4.11 requires Fréchet differentiability for $G_{c}$ with respect to $\partial D_{x_{0}, r}$, which will be established in the following lemma.

Lemma 4.14. Let $r \in T \cap V_{a d}$ such that $\partial D_{x_{0}, r} \in \mathcal{U}_{a d}^{\prime}$. Then $G_{c}$ is Fréchet differentiable with respect to $\partial D_{x_{0}, r}$ in the sense of Section 3.4 and Theorem 4.8 , 
and the Fréchet derivative is given by

$$
G_{c}^{\prime}[r ; h]=2 \int_{0}^{2 \pi}\left(r^{\prime \prime}(t)-r(t)\right)\left(h^{\prime \prime}(t)-h(t)\right)+2 r^{\prime}(t) h^{\prime}(t) d t
$$

for $h \in T$.

Proof. From (4.32) we see that the penalty term only depends on the boundary via its integrand, which is a polynomial expression in the radial function and its first two derivatives. According to Theorem 4.8 Fréchet differentiability in the sense of Section 3.5 is equivalent to Fréchet differentiability with respect to $r$. Thus, the statement of the Lemma follows by differentiating the integrand.

\subsection{A level set approach to the Geometric Op- timization Problem}

In this section we propose a second approach to numerically solve the Geometric Optimization Problem 3.3. It is based on the so-called level set idea, which was invented in 1988 by Osher and Sethian in their seminal paper [56].

The idea has been widely used in Stefan problems $[15,16]$ and in the modelling of chrystal growth $[32,67]$. Rather recently, level sets have been successfully used in computational fluid dynamics [71, 77], computer vision [62], and structural optimization [5, 68]. Level sets have been introduced to inverse problems in 1996 in a paper by Santosa [63], where also the idea has been prevalent to obtain the velocity field from differentiation with respect to the boundary. This idea has been studied further in the context of eigenvalue problems for the Laplacian by Santosa and Osher in [55]. Other approaches to use level set methods in the context of inverse problems are mainly due to Burger (see $[6,12,13])$. The combination of level set methods and boundary integral equations has not been studied yet in the mathematical community, only in the work of Sethian and Strain [67] from 1992 the velocity field is derived from the boundary representation of a heat potential, but without the idea of differentiating with respect to the boundary. Nevertheless, there is a series of papers in the engineering community by Ferrayé, Dauvignac and Pichot [26, 27, 28, 29], where the inverse scattering problem for perfectly conducting media is treated using a combination of boundary integral methods and level set methods. We will discuss the conceptual relation between this approach and our work in the discussion in Section 6.1.

Firstly, we will give a concise introduction to the idea of level set methods without going too much into details about the theory on which they are based. We will then describe how the general idea of level set evolution can be related with and 
used for the Geometric Optimization Problem from Chapter 3. Subsequently, we will discuss various issues of the numerical realization of level set methods in general and also focus on questions that are specific to the problem at hand. We will especially describe and examine how to resolve the 0-level set in the context of a level set method and how to derive a velocity field from a 'boundary' velocity. Both issues have not been discussed in the literature on level set methods so far. We will propose newly developed algorithms which realize these issues exploiting a kind of duality relation between them.

Let us begin with a brief description of level set methods and their origins. We first of all give a rigorous definition of level sets themselves.

Definition 4.15 (Level sets). Let $\phi \in C\left(\mathbb{R}^{2} \times[0, \infty)\right)$ be a real-valued function. Then for $t \geq 0$ and $c \in \mathbb{R}$ the set

$$
C_{t, c}(\phi):=\left\{x \in \mathbb{R}^{2}: \phi(x, t)=c\right\}
$$

is called the c-level set of $\phi$ at time $t$.

By the implicit function theorem the set $C_{t, c}(\phi)$ is of class $C^{1}$ provided $\phi$ is continuously differentiable. In this way we can relate a desired regularity of the level set $C_{t, c}(\phi)$ to a required regularity of $\phi$. Although in general each $c$-level set of $\phi$ has equal rights, we will only consider the 0-level set for conceptual and computational simplicity.

Level sets, being motivated from a geometric point of view, appear as the level sets of the time-dependent solution to certain evolution equations that are called level set equations. We will introduce the evolution equations in the following.

Problem 4.16. Let $C \subset \mathbb{R}^{2}$ be a curve in $\mathbb{R}^{2}$, and let $V: \mathbb{R}^{2} \times[0, \infty) \rightarrow \mathbb{R}^{2}$ be a (smooth) vector field.

Find a function $\phi: \mathbb{R}^{2} \times[0, \infty) \rightarrow \mathbb{R}$ satisfying the Initial Value Problem given through the advection equation

$$
\frac{\partial \phi(x, t)}{\partial t}+\langle V(x, t), \operatorname{grad} \phi(x, t)\rangle=0
$$

together with the initial condition

$$
C_{0,0}(\phi)=C
$$

Let us consider (4.35) in more detail. Concentrating on the 0-level set, we observe that the velocity field $V$ induces spatial movement of the points on the 0 -level set of $\phi$, which is balanced by the temporal evolution of $\phi$. In this sense $V$ propagates the level sets of $\phi$ in space as we progress in time. Furthermore, one can observe that a general velocity field $V$ contains information that is irrelevant for the evolution of the 0-level set of $\phi$. We illustrate this in the following example. 
Example 4.17. Consider Problem 4.16 with initial curve

$$
C:=\left\{x \in \mathbb{R}^{2}:|x|=1\right\}
$$

being the unit circle, and a velocity field $V$ that satisfies

$$
V(x, t)=\left(\begin{array}{r}
-\sin \vartheta \\
\cos \vartheta
\end{array}\right) \quad \text { for all } t \geq 0
$$

and for all $x=(\cos \vartheta, \sin \vartheta)^{T}$ with $\vartheta \in[0,2 \pi)$. Then a smooth solution to the initial values is given by

$$
\phi(x, 0)=|x|^{2}-1, \quad x \in \mathbb{R}^{2} .
$$

Calculating the gradient of $\phi(\cdot, 0)$, we obtain that

$$
\langle V(x, 0), \operatorname{grad} \phi(x, 0)\rangle=2\left\langle\left(\begin{array}{r}
-\sin \vartheta \\
\cos \vartheta
\end{array}\right),\left(\begin{array}{c}
\cos \vartheta \\
\sin \vartheta
\end{array}\right)\right\rangle=0
$$

for all $x=(\cos t, \sin t)^{T}$ on the 0-level set. In view of (4.35) this implies that

$$
\frac{\partial \phi(x, 0)}{\partial t}=0 \quad \text { for all } x=(\cos t, \sin t)^{T}
$$

which means that the 0-level set of $\phi$ does not change in time.

Generally speaking, we can summarize the observation of Example 4.17 as follows. The gradient of the solution $\phi$ is orthogonal (or normal) to the level sets of $\phi$. Hence, (4.35) does not 'see' velocity fields $V$ that are tangential to the level sets. Consequently we can split any (smooth) velocity field $V$ on $\mathbb{R}^{2}$ according to

$$
V=V_{n} \cdot N+V_{t} \cdot T
$$

where

$$
N(x, t):=\frac{\operatorname{grad} \phi(x, t)}{|\operatorname{grad} \phi(x, t)|}, \quad T(x, t):=\frac{[\operatorname{grad} \phi(x, t)]^{\perp}}{|\operatorname{grad} \phi(x, t)|},
$$

are the normal, respectively tangential, field of $\phi$, and $V_{n}: \mathbb{R}^{2} \rightarrow \mathbb{R}, V_{t}: \mathbb{R}^{2} \rightarrow \mathbb{R}$ are the normal, respectively tangential, component of $V$. Plugging the representation (4.37) into (4.35), we see that (4.35) is equivalent to the so-called level set equation

$$
\frac{\partial \phi(x, t)}{\partial t}+V_{n}(x, t)|\operatorname{grad} \phi(x, t)|=0
$$

The solution $\phi$ to Problem 4.16 with the advection equation (4.35) replaced by the level set equation (4.39) is accordingly called level set function. 
We would like to remark here that in the literature also (4.35) is sometimes referred to as the level set equation. This is due to the fact that in the cases of externally generated velocity fields, such as in fluid flow, (4.35) is used directly for level set methods, as $V$ is more easily accessible than its normal component. Opposed to that, (4.39) is used whenever the velocity field is internally or selfgenerated, as it happens for example in applications involving mean curvature flow. We refer to [54] and the references therein for a closer study of the differences between (4.35) and (4.39). For the application of level set methods to the Geometric Optimization Problem 3.3 we will only employ (4.39), but we will have to turn back to (4.35) during our discussions in order to illustrate various phenomena.

Let us proceed with a rough overview of the theoretical backgrounds of level set methods that render the numerical schemes mathematically sound. From a systematic point of view the level set equation (4.39) can be seen as a particular example for a Hamilton-Jacobi equation, which is generally given in the form

$$
\phi_{t}+H(\operatorname{grad} \phi)=0
$$

for some function $H$ depending on the spatial gradient of $\phi$. Furthermore, there is a connection to hyperbolic conservation laws which can be formulated directly in one spatial dimension in the following example.

Consider the one-dimensional Hamilton-Jacobi equation

$$
\phi_{t}+H\left(\phi_{x}\right)=0
$$

and differentiate formally with respect to the spatial variable $x$ to obtain the equation

$$
u_{t}+[H(u)]_{x}=0
$$

for the slope $u=\phi_{x}$, which is a scalar conservation law. In this sense, solutions to Hamilton-Jacobi equations and hyperbolic conservation laws correspond.

Based on this correspondence we may draw several conclusions on the solution of Problem 4.16. First, we note that the solution to Problem 4.16 is by no means unique, nonetheless as we only impose incomplete initial conditions by prescribing the 0 -level set of $\phi$ at the initial time-level $t=0$. Furthermore, the above correspondence reveals an additional argument for non-uniqueness, since hyperbolic conservation laws can have non-unique solutions. In this context, entropy conditions have to be imposed to pick out the 'correct' or 'physically relevant' solution (see, for example [65]). Correspondingly, for Hamilton-Jacobi equations a theory of weak solutions or vanishing viscosity solutions has been developed, in which the solutions to equations of the form of (4.40) may be interpreted as the limit of solutions $u^{\varepsilon}$ to the equation

$$
u_{t}^{\varepsilon}+H\left(\operatorname{grad} u^{\varepsilon}\right)=\varepsilon \Delta u^{\varepsilon}
$$


as $\varepsilon \rightarrow 0$ in the right-hand side. For a detailed study of the theory of vanishing viscosity solutions to Hamilton-Jacobi equations we refer to [20, 21, 25].

\section{How are level set methods realized in general?}

We will not attempt to solve the Problem 4.16 analytically, since we are not interested in the level set function $\phi$ as a whole, but just in its steady state part or, to be even more precise, in the 0-level set of that steady state part. Consequently, we only strive to solve Problem 4.16 numerically, by propagating the initial choice for $\phi$ according to (4.39) using numerical methods that have been developed for hyperbolic conservation laws. In the following algorithm we present a generally valid procedure for the numerical realization of a level set method by means of the particular example given by the Geometric Optimization Problem 3.3. The central ideas and algorithms that build the foundations of the individual steps of Algorithm 4.18 will be discussed in detail in the subsequent paragraphs of this section.

Algorithm 4.18 (Level Set Algorithm). Let $\Gamma \subset \mathbb{R}^{2}$ be an open arc of class $C^{3}$ in the sense of Definition 1.5, and let $I \neq 0, \mu_{e}>0$ and $\mu_{i}>0, i=1, \ldots, n$, be given. Furthermore, let $C \in \mathcal{U}_{a d}$ be the boundary curve of some admissible domain $D_{0}$ in the sense of Definition 3.1. Then the level set algorithm works as follows.

Initialization Step: Using $C$ as an initial curve for the time-level $t=0$, we first build a level set function $\phi(\cdot, 0)$ that satisfies the initial conditions (4.36). Then we repeat the following steps until the functional (3.4) stabilizes.

1. Contouring Step: Using a contouring algorithm, we resolve the 0-level set $C_{t, 0}$ in the sense that we calculate an ordered set of points $\left\{z_{1}, \ldots, z_{n}\right\}$ approximating $C_{t, 0}$. Exploiting properties of the level set function we also compute the normal to $C_{t, 0}$ and the curvature at these points.

2. Evaluation and Descent Direction Step: We interpret the 0-level set $C_{t, 0}(\phi)$ as the boundary of the domain

$$
D_{t}:=\left\{x \in \mathbb{R}^{2}: \phi(x, t)<0\right\}
$$

and use the calculated boundary information from the previous step to numerically solve the Boundary Value Problem 2.1 for the geometry $\left(\Gamma, D_{t}\right)$. From the solution $u$ to Problem 2.1, we can evaluate the functional $F\left(C_{t, 0}\right)$ of the Geometric Optimization Problem 3.3. Furthermore, we can employ the sensitivity analysis of Chapter 3 to calculate Fréchet derivative

$$
F^{\prime}\left[C_{t, 0} ; \alpha_{i} N\right], \quad i=1, \ldots, n,
$$


for the directions $\alpha_{i} N$, as it has been introduced in Theorem 3.36. Here, $N$ is given by (4.38) and the $\alpha_{i}: C_{t, 0} \rightarrow[0,1] \subset \mathbb{R}$ are smooth functions that form a partition of unity for $C_{t, 0}$ and satisfy $\alpha_{i}\left(z_{j}\right)=\delta_{i j}$.

(According to Section 3.4 the perturbations $\alpha_{i} N$ are defined on the boundary only. But they can be extended to compactly supported vector fields on $\mathbb{R}^{2}$ that meet the requirements of Section 3.4. In [39], Hohage has proposed Whitney-type extension operators for a similar situation.)

3. Extension and Re-Distancing Step: The Fréchet derivative of $F$ at the current boundary $C_{t, 0}$ in direction $\alpha_{i} N$ indicates whether $F$ is increasing or decreasing when the current boundary $C_{t, 0}$ is perturbed in direction $\alpha_{i} N$. In this sense we can interpret the Fréchet derivative $F^{\prime}\left[C_{t, 0} ; \alpha_{i} N\right]$ for the directions $\alpha_{i} N$ as a 'boundary' velocity via

$$
F_{n}=-\sum_{i=1}^{n} F^{\prime}\left[C_{t, 0} ; \alpha_{i} N\right] \alpha_{i} N
$$

which induces a perturbation of the current boundary that decreases the functional $F$. We then extend $F_{n}$ as the normal part $V_{n} \cdot N$ of a velocity field in the spirit of (4.37). Simultaneously, we re-initialize the level set function $\phi(\cdot, t)$ to a signed distance function using a re-distancing algorithm.

4. Propagation Step: Driven by the normal velocity $V_{n}$ we propagate the 0 -level set in a last step in artificial time. As $V_{n}$ aims at decreasing the cost functional $F$, we expect that $F$ is indeed decreasing if we perform a discrete time-step. We therefore numerically approximate the evolution of (4.39) by performing a time-stepping algorithm. That is, we use an explicit forward Euler time discretization with an appropriately chosen step size $\Delta t$, and execute one time step. For the spatial approximation of $\operatorname{grad} \phi(\cdot, t)$ we employ techniques such as upwind differencing that have been especially developed for hyperbolic conservation laws. In this way we obtain an approximation for the level set function $\phi$ at the next time-level $t+\Delta t$.

The numerical implementation of the level set function $\phi$ and the normal component $V_{n}$ of the velocity field work as follows. We discretize an a priori chosen computational domain with a uniform mesh $x_{i, j}$ with mesh size $h$ in both directions. Then the discretized version of $\phi$ at time $t_{k}$ is given as a matrix $\phi^{k}$ of discrete values $\phi^{k}\left(x_{i, j}\right)$. Similarly $V_{n}$ is discretized as matrix that contains the values $V_{n}\left(x_{i, j}\right)$.

If realized naively, this method is inefficient, since the values for $\phi$ are close to zero only in a comparatively small region of the computational domain. As we neither expect nor want additional components of the 0-level set to occur in 
regions far away from the existing 0-level set, we discretize $\phi$ and $V_{n}$ only in a narrow band around the existing 0-level set, and assign either undefined values or $\pm \infty$ to all grid points that are further away from the existing 0-level set than some prescribed bandwidth. The idea of discretizing only a narrow band around the current 0-level set is due to Adalsteinsson and Sethian [1]. We observe in particular that the 0-level set never reaches regions with undefined values for $\phi$ and $V_{n}$, as in the course of the re-distancing algorithm we automatically adjust the narrow band to be centered around the 0-level set of the current time-level.

\section{How to initialize a level set function for a given geometry?}

This issue has to be adressed in the initial phase of a level set method, where in terms of Problem 4.16 we have to find a level set function $\phi$ for the initial timelevel $t=0$ that satisfies the initial conditions (4.36). As the literature on level set methods is rather vague on this topic, we describe four off-hand approaches. The first two are analytic methods, of which the first one may also be used directly for numerical implementation. The second approach displays several drawbacks, especially in its straightforward numerical implementation. Hence, we have also included the third and fourth approach, which are numerical realization of the second approach that overcome these drawbacks.

1. Let the simple, closed curve $C$ be described as the solution to an algebraic equation of the form

$$
p\left(x_{1}, x_{2}\right)=c, \quad x=\left(x_{1}, x_{2}\right) \in \mathbb{R}^{2} .
$$

Then set

$$
\phi(x, 0):= \pm\left(p\left(x_{1}, x_{2}\right)-c\right), \quad x=\left(x_{1}, x_{2}\right) \in \mathbb{R}^{2}
$$

to obtain the corresponding level set function. Depending on whether the domain $D$ enclosed by $C$ is meant to be $\left\{x \in \mathbb{R}^{2}: p\left(x_{1}, x_{2}\right)<c\right\}$ or $\left\{x \in \mathbb{R}^{2}: p\left(x_{1}, x_{2}\right)>c\right\}$, the corresponding sign has to be chosen for $\phi$.

2. Let the simple, closed curve $C$ be given in terms of a parametrization $z:[0,2 \pi) \rightarrow \mathbb{R}^{2}$, and let $D$ be the domain enclosed by $C$.

Then define the double-layer potential $\mathcal{T}^{D}$ on $C=\partial D$ with density $\varphi \equiv 1$ according to Definition 1.11 and set

$$
\phi(x, 0):=\left(\mathcal{T}^{D} 1\right)(x)+\frac{1}{2},
$$

where we consider the value of $\left(\mathcal{T}^{D} 1\right)$ for $x \in \partial D$ by its direct value and use that the double-layer potential with a constant density is constant in the interior, respectively exterior of its domain (see [47], Example 6.16). 
3. Let the simple, closed curve $C$ be given in terms of a parametrization $z:[0,2 \pi) \rightarrow \mathbb{R}^{2}$, and let $D$ be the domain enclosed by $C$. Assume that

- the grid is so fine that the distance between the intersection points of $C$ and any grid line is strictly larger than the mesh size $h$.

- the curve $C$ is nowhere tangent to the grid lines.

Then for each grid line $L$ (vertical or horizontal) calculate the intersection points $s$ between $L$ and $z$ as well as the unit normal $\nu_{s}$ at the intersections pointing into the exterior of $D$. In the special case of $s$ being a grid point itself, we move $s$ by $h \cdot 10^{-2}$ along $L$ in any direction. (Observe that this does not change the property that between any two intersections of $C$ of the same grid line $L$ there is at least one grid point.) Then we assign to the two uniquely determined grid points $x_{+}$and $x_{-}$on $L$ being next to $s$ the value

$$
\left\langle\nu_{s}, x_{ \pm}-s\right\rangle
$$

for $\phi$, provided $\phi$ has not already been assigned with a smaller absolute value. In this way we assign to each grid point that is 'next to an intersection' a distance value that is at most the distance between the grid point and the nearest intersection.

4. Let the simple, closed curve $C$ be piecewise linear, i.e. $D$ is a polygon with corners given by $\left\{x_{1}, \ldots, x_{n}\right\}$. Assume that

- the grid is so fine that the distance between the intersection points of $C$ and any grid line is strictly larger than the mesh size $h$. (This implies in particular, that no side of the polygon lies on a grid line.)

- $x_{i} \notin L$ for any grid line $L$ and any $i=1, \ldots, n$.

Then we can proceed as in the third method.

The first method is extremely simple to implement as long as one is content with simple geometries such as circles or ellipses for an initial curve $C$. It will be used throughout the examples of Section 5.3. The second approach is of nearly no practical and also just of little theoretical importance. From the practical side one has to bear in mind that the double-layer potential will in general be approximated via a quadrature rule that becomes more and more inaccurate as the evaluation point tends to $\partial D$. Precisely there, the sign of $\phi$ has to be most reliable, which renders this method numerically unsatisfactory. From a theoretical point of view the level set function obtained by the second approach is of rather little value as the only information it contains is basically the information whether a point is in $D$ or not. This can be seen best from the equation

$$
\left(\mathcal{T}^{D} 1\right)(x)+\frac{1}{2}=\frac{1}{2}\left(\chi_{\mathbb{R}^{2} \backslash \bar{D}}(x)-\chi_{D}(x)\right) .
$$


The third and fourth approach may be viewed as more accurate versions of the second approach. In the third method we still assume to know the parametrization of the boundary exactly, whereas in the fourth method we are only given a polygon which may be interpreted as an approximation by linear splines. The difference of both approaches to the second method is that we treat the grid points nearest to the curve $C$ as accurately as possible, and equip the discrete values of $\phi$ with as much information about the distance from the point to the curve $C$ as possible.

One might pose the objection against these two methods that they only care about the grid points nearest to the curve $C$ and assign no values to grid points that are further away. Fortunately, this objection can be ruled out with the observation, that the assignment of distance values to grid points that are further away can be subsumed under the issue of re-distancing, which will be treated in one of the following paragraphs.

Secondly, we remark that for any reasonable choice for an initial curve $C$ a mesh size and shift can be obtained such that the technical assumptions on the third and fourth method can also be neglected.

The above algorithms are concerned with creating a level set function for a simply connected, bounded domain. The generalization to domains with an arbitrary finite number of connected components is straightforward. After having built level set functions $\phi_{1}, \ldots, \phi_{n}$ for each connected component, we define

$$
\phi(x):=\min \left\{\phi_{1}(x), \ldots, \phi_{n}(x)\right\} .
$$

As the components are disjoint, we have for $x \in D_{i}=\left\{y \in \mathbb{R}^{2}: \phi_{i}(y, 0)<0\right\}$ that $\phi_{j}(x, 0)>0$ for all $j \neq i$ as well as $\phi_{i}(x, 0)<0$. Consequently $\phi(\cdot, 0)$ satisfies

$$
\begin{array}{ll}
\phi(x, 0)<0 & \text { for all } x \in \bigcup_{i=1}^{n} D_{i}, \\
\phi(x, 0)>0 & \text { for all } x \in \mathbb{R}^{2} \backslash\left(\bigcup_{i=1}^{n} \bar{D}_{i}\right), \\
\phi(x, 0)=0 & \text { for all } x \in \bigcup_{i=1}^{n} \partial D_{i} .
\end{array}
$$

We close this paragraph with the remark that the method described above also works if the subdomains $D_{i}$ are not disjoint. In this case we can use the method to create initial curves of more elaborate nature from rather simple ones.

Apart from this, multiple level set functions can also be used to distinguish material properties that vary within the domain. We refer to [14, 17, 72] for further studies on this idea. 


\section{How to do the contouring?}

Contouring is a task which level set methods normally seek to avoid as the evolution of the 0-level set of $\phi$ is regarded as a side effect of the evolution of $\phi$ as a whole, although it is the only evolution we are concerned about. This philosophy of not resolving the 0-level set works fine as long as there is a more or less natural way to define a velocity field in all of $\mathbb{R}^{2}$ that induces the correct evolution. We will see in the paragraph on velocity extension on page 141 that this requirement has been relaxed in the literature, in the sense that the velocity field has to be given only in a small neighbourhood of the 0-level set. But still, off-front evaluation of the velocity field is one of the keys to level set methods.

Unfortunately, there are numerous applications, where only on the 0-level set itself the definition of a velocity makes sense. Examples can be found in etching processes (see [2]) or Stefan problems (see [15]). The Geometric Optimization Problem 3.3 also presents an example of that kind. In Chapter 3 we have introduced the functional $F$ as a measure for the deviation of the current distribution on $\Gamma$ from being constant. This deviation has been interpreted as a function of the boundary $\partial D$, and the Fréchet derivatives of $F$ in direction $\alpha_{i} N$ have been interpreted as a 'boundary' velocity in (4.42).

The Geometric Optimization Problem 3.3 displays thereby that this class of applications, where off-front evaluation of the velocity field is undefined, provides two problems. Not only that one needs to find a way how to extend the 'boundary' velocity to a velocity field on all of $\mathbb{R}^{2}$ which induces the correct evolution, but one also needs to know the 0-level set exactly in order to calculate this 'boundary' velocity.

Level set methods in general provide only a discrete approximation to the level set function on the grid points of a uniform mesh. In the following we will use this information in the spirit of the Fast Marching Method, which will be described in detail in the paragraph on re-distancing, in order to find a finite set of points $\left\{\bar{x}^{(1)}, \ldots, \bar{x}^{(n)}\right\}$ on the 0 -level set that forms a linear spline approximation to it. We will furthermore use properties of the level set function to recover more information about the 0-level set than just its position. In particular, we will derive formulae for the normal and the curvature at the points $\bar{x}^{(k)}$. In this way we obtain all the information the boundary integral formulation of the Boundary Value Problem 2.1 and the Geometric Optimization Problem 3.3 require in order to numerically calculate the Fréchet derivatives of $F$ in direction $\alpha_{i} N$.

Assume that we are given the discretized version $\phi^{n} \approx \phi(\cdot, t)$ of the level set function $\phi$ at time-level $t$, and assume that no grid point is on the 0-level set, i.e. $\phi^{n}\left(x_{i, j}\right) \neq 0$. Then there is a well-defined band of grid cells through which the 0-level set is running. This is illustrated in Figure 4.4.

Observe in particular that it is sufficient for this that the level set function is defined only on the grid points adjacent to the sides of the grid cell which the 


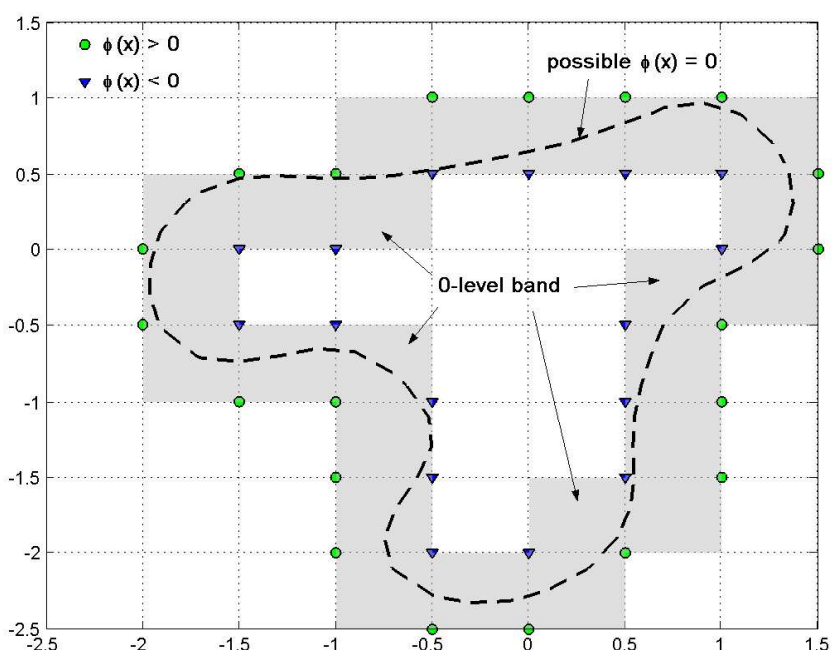

Figure 4.4: Possible position of the 0-level set for a given level set function $\phi$

0-level set intersects. (In Figure 4.4 these grid points are marked with green circles when $\phi$ is positive, they are marked with blue triangles when $\phi$ is negtive.) Focussing on one particular grid cell and disregarding possible rotations as well as a possible multiplication of the level set function with the factor -1 , the position of the 0-level set in that grid cell is given by one of the schematics of Figure 4.5.

a)

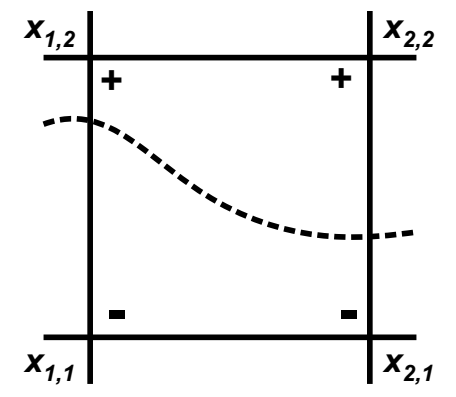

b)

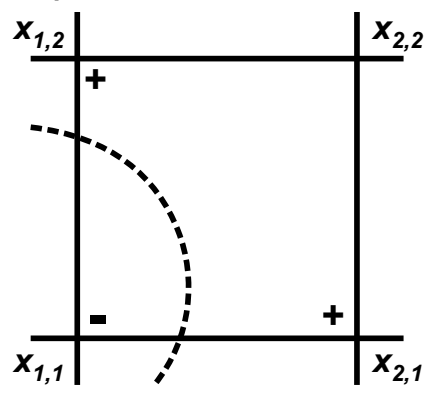

c)

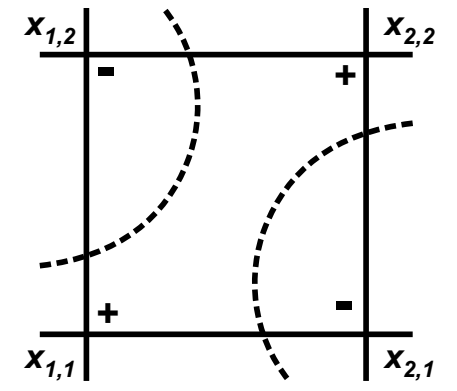

Figure 4.5: Schematics for the possible position of the 0-level set in a grid cell

The aim of a contouring algorithm now is to construct one, respectively two, points $\bar{x}$ in this grid cell such that the resulting linear spline curve is a good approximation to the 0-level set.

We begin with the treatment of the case displayed in Figure 4.5 a). Here, we have distance values at all four grid points that build the corners of the grid cell, the two at the upper corners being positive, the two at lower corners being negative. We illustrate the following procedure in Figure 4.6. By linear interpolation along the vertical grid lines we can find two points $z_{1}$ and $z_{2}$ where the linear approximation to $\phi$ vanishes. Connecting $z_{1}$ and $z_{2}$ by a line we obtain a line segment 


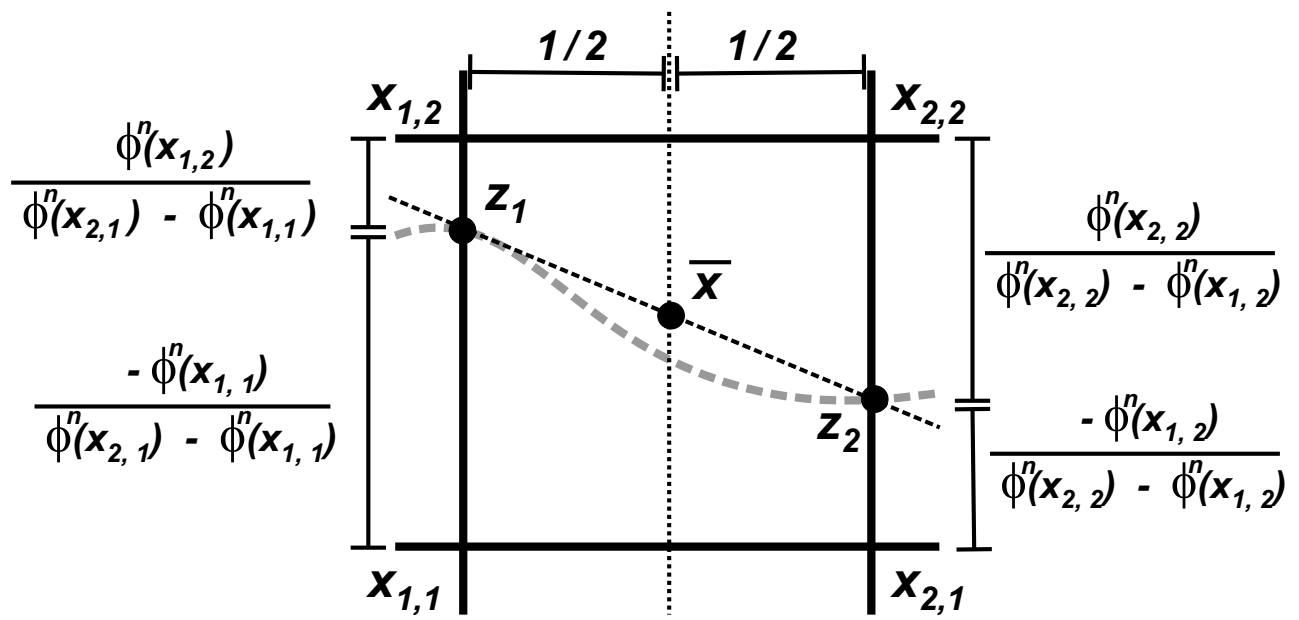

Figure 4.6: Illustration for the construction of $\bar{x}$ in the case of Figure 4.5 a)

in the grid cell which is an approximation to the 0-level set. As there is no more information in the values of $\phi$ at the corners, in horizontal direction any point on the constructed line can be used as a point approximating the 0-level set. For symmetry reasons we therefore choose the point in the middle of the particular line. The so constructed point $\bar{x}$ can be described as a convex combination of the corners. If we assume that the lower left corner is indexed with $(1,1)$ we obtain

$$
\bar{x}:=a_{1,1} x_{1,1}+a_{2,1} x_{2,1}+a_{1,2} x_{1,2}+a_{2,2} x_{2,2},
$$

where the convex weights are given by

$$
\begin{aligned}
a_{1,1}=\frac{\phi^{n}\left(x_{1,2}\right)}{2\left(\phi^{n}\left(x_{1,2}\right)-\phi^{n}\left(x_{1,1}\right)\right)}, & a_{1,2} & =\frac{-\phi^{n}\left(x_{1,1}\right)}{2\left(\phi^{n}\left(x_{1,2}\right)-\phi^{n}\left(x_{1,1}\right)\right)}, \\
a_{2,1}=\frac{\phi^{n}\left(x_{2,2}\right)}{2\left(\phi^{n}\left(x_{2,2}\right)-\phi^{n}\left(x_{2,1}\right)\right)}, & a_{2,2} & =\frac{-\phi^{n}\left(x_{2,1}\right)}{2\left(\phi^{n}\left(x_{2,2}\right)-\phi^{n}\left(x_{2,1}\right)\right)} .
\end{aligned}
$$

Furthermore, we can determine an approximation to the gradient of $\phi$ on the grid cell for each corner by

$$
\begin{aligned}
\operatorname{grad} \phi^{n}\left(x_{1,1}\right) & \approx\left(\frac{\phi^{n}\left(x_{2,1}\right)-\phi^{n}\left(x_{1,1}\right)}{h}, \frac{\phi^{n}\left(x_{1,2}\right)-\phi^{n}\left(x_{1,1}\right)}{h}\right)^{T}, \\
\operatorname{grad} \phi^{n}\left(x_{2,1}\right) & \approx\left(\frac{\phi^{n}\left(x_{2,1}\right)-\phi^{n}\left(x_{1,1}\right)}{h}, \frac{\phi^{n}\left(x_{2,2}\right)-\phi^{n}\left(x_{2,1}\right)}{h}\right)^{T}, \\
\operatorname{grad} \phi^{n}\left(x_{1,2}\right) & \approx\left(\frac{\phi^{n}\left(x_{1,2}\right)-\phi^{n}\left(x_{1,1}\right)}{h}, \frac{\phi^{n}\left(x_{2,2}\right)-\phi^{n}\left(x_{1,2}\right)}{h}\right)^{T}, \\
\operatorname{grad} \phi^{n}\left(x_{2,2}\right) & \approx\left(\frac{\phi^{n}\left(x_{2,2}\right)-\phi^{n}\left(x_{1,2}\right)}{h}, \frac{\phi^{n}\left(x_{2,2}\right)-\phi^{n}\left(x_{2,1}\right)}{h}\right)^{T} .
\end{aligned}
$$


Consequently, the approximation to the gradient of $\phi$ is derived using the same convex combination as above, and reads

$$
\begin{aligned}
\operatorname{grad} \phi^{n}(\bar{x}):= & a_{1,1} \operatorname{grad} \phi^{n}\left(x_{1,1}\right)+a_{2,1} \operatorname{grad} \phi^{n}\left(x_{2,1}\right) \\
& +a_{1,2} \operatorname{grad} \phi^{n}\left(x_{1,2}\right)+a_{2,2} \operatorname{grad} \phi^{n}\left(x_{2,2}\right) .
\end{aligned}
$$

Naturally, the gradient of $\phi$ is perpendicular to the level lines of $\phi$ and points into the direction of the steepest ascent. Hence, it coincides with the exterior normal to the boundary $\partial D=C_{t, 0}(\phi)$ of $D=\left\{x \in \mathbb{R}^{2}: \phi(x, t)<0\right\}$.

Furthermore the curvature of the 0-level set is a quantity which is inherent in the level set function. From geometry we know that the curvature of a curve is given as the divergence of the unit normal. In terms of level set functions this means that

$$
\kappa(x)=\operatorname{div}\left(\frac{\operatorname{grad} \phi}{|\operatorname{grad} \phi|}\right) .
$$

Using central differences $\phi_{x}, \phi_{y}, \phi_{x x}, \phi_{x y}, \phi_{y y}$ to approximate the first and second order partial derivatives of $\phi$ at the corners $x_{i, j}$ we can calculate an approximation to (4.44) on each grid point as

$$
\kappa\left(x_{i, j}\right) \approx \frac{\phi_{x x}\left(\phi_{y}\right)^{2}-2 \phi_{x} \phi_{y} \phi_{x y}+\phi_{y y}\left(\phi_{x}\right)^{2}}{\left(\left(\phi_{x}\right)^{2}+\left(\phi_{y}\right)\right)^{\frac{3}{2}}},
$$

and thus derive the approximation for

$$
\kappa(\bar{x}):=a_{1,1} \kappa\left(x_{1,1}\right)+a_{2,1} \kappa\left(x_{2,1}\right)+a_{1,2} \kappa\left(x_{1,2}\right)+a_{2,2} \kappa\left(x_{2,2}\right) .
$$

The reason for using central differences for the curvature opposed to one-sided differences for the gradient approximation is that the curvature is a diffusive term while the gradient is advective.

Summarizing the above, we have found a point $\bar{x}$ approximating the 0 -level set in a grid cell of the type of Figure 4.5 a) together with approximations for $\nu(\bar{x})$ and $\kappa(\bar{x})$.

Next, we examine the situation of Figure $4.5 \mathrm{~b}$ ). Here, differently to a) distance values are available only for the corners $x_{1,1}, x_{1,2}$ and $x_{2,1}$. Proceeding by linear interpolation as in case a) finally leads to a point $\bar{x}$ that is closer to $x_{1,1}$ as the distance value $\phi^{n}\left(x_{1,1}\right)$ indicates. Hence we choose a different approach. As we only have distance values for $x_{1,1}, x_{1,2}$ and $x_{2,1}$, only the approximation to the gradient (4.43) in $x_{1,1}$ is well-defined. Hence, we set

$$
\bar{x}:=x_{1,1}-\phi^{n}\left(x_{1,1}\right) \frac{\operatorname{grad} \phi^{n}\left(x_{1,1}\right)}{\left|\operatorname{grad} \phi^{n}\left(x_{1,1}\right)\right|}
$$

as well as

$$
\operatorname{grad} \phi^{n}(\bar{x}):=\operatorname{grad} \phi^{n}\left(x_{1,1}\right)
$$




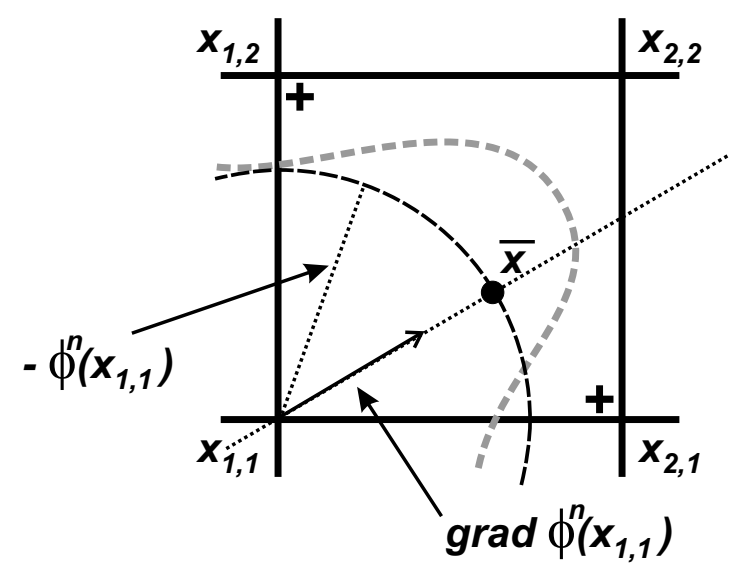

Figure 4.7: Illustration for the construction of $\bar{x}$ in the case of Figure 4.5 b)

Note that in (4.46) the value of $\phi^{n}\left(x_{1,1}\right)$ is negative. Therefore $\bar{x}$ lies indeed inside the grid cell and approximates the 0-level set correctly. The procedure is illustrated in Figure 4.7.

We also have to assign a curvature value to $\bar{x}$. As mentioned above the curvature is of a diffusive nature, so that it is not sensible to use only the curvature approximation from $x_{1,1}$ for $\bar{x}$. Instead, we use a convex combination of the curvature approximations from $x_{1,1}, x_{1,2}$ and $x_{2,1}$ in the spirit of case of Figure 4.5 a). We set

$$
\kappa(\bar{x}):=b_{1,1} \kappa\left(x_{1,1}\right)+b_{2,1} \kappa\left(x_{2,1}\right)+b_{1,2} \kappa\left(x_{1,2}\right),
$$

where $\kappa\left(x_{1,1}\right), \kappa\left(x_{2,1}\right)$, and $\kappa\left(x_{1,2}\right)$ are obtained from (4.45), and where the convex weights are given by

$$
\left(b_{2,1}, b_{1,2}\right)=-\frac{\phi^{n}\left(x_{1,1}\right)}{h\left|\operatorname{grad} \phi^{n}\left(x_{1,1}\right)\right|} \operatorname{grad} \phi^{n}\left(x_{1,1}\right), \quad b_{1,1}=1-b_{2,1}-b_{1,2} .
$$

Finally, we consider the case of Figure $4.5 \mathrm{c}$ ). We first observe that this case is of a rather philosophical nature as the topology of the 0-level is not determined from the values of $\phi$ at the corners. The possible topologies are illustrated in Figure 4.8.

We see that the resolution of the mesh is just not fine enough to resolve the correct topology. In such a situation we assume that the 0-level set is given by the right-hand side option as this ensures that the region where $\phi$ is positive remains connected. Thereby we try to avoid the formation of holes in the region where $\phi$ is negative, and which is interpreted as the domain $D$.

As soon as this choice has been made, we can treat the disconnected parts of the 0-level set separately. This yields twice the case of Figure $4.5 \mathrm{~b}$ ), which then is treated as described above.

If we proceed as described above for all grid cells through which the 0-level set is running, we obtain a set of points $\left\{\bar{x}^{(1)}, \ldots, \bar{x}^{(n)}\right\}$ that is roughly of the same 

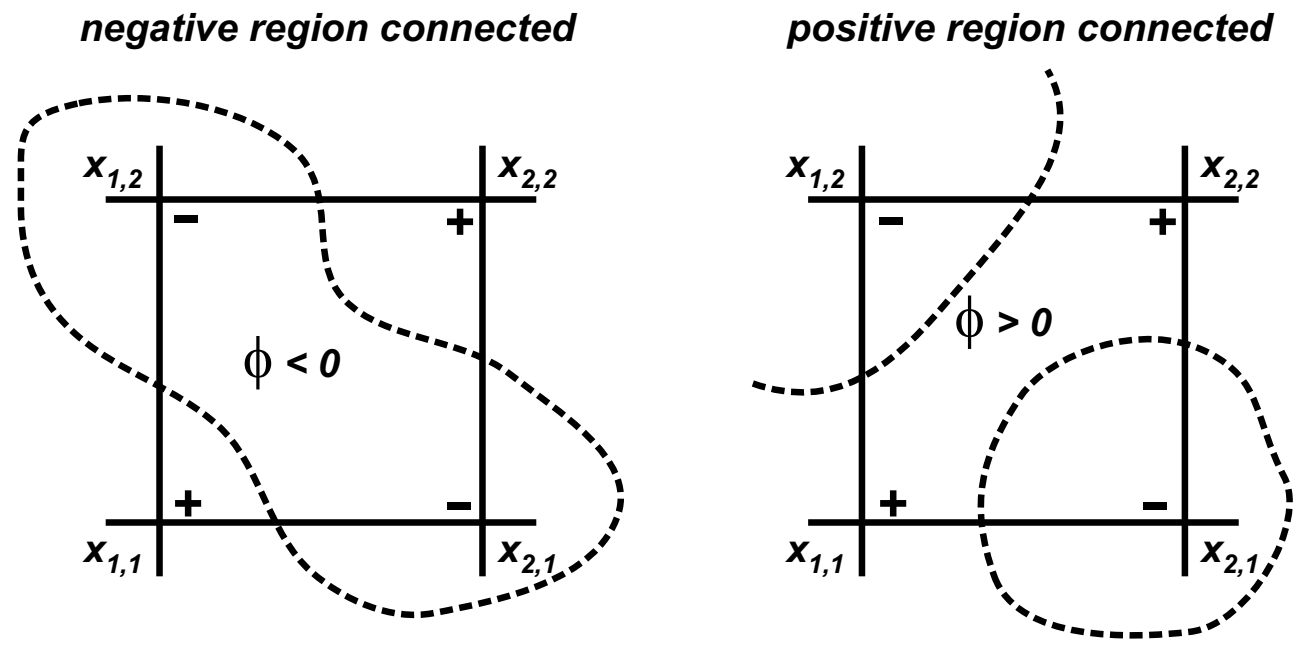

Figure 4.8: Possible topologies for the regions $\phi>0$ and $\phi<0$ in the case of Figure $4.5 \mathrm{c}$ )

number as the number of grid cells through which the 0-level set is running. The set approximates the 0-level set yielding, together with the approximations $\left\{\nu\left(\bar{x}^{(1)}\right), \ldots, \nu\left(\bar{x}^{(n)}\right)\right\}$ for the normal and $\left\{\kappa\left(\bar{x}^{(1)}\right), \ldots, \kappa\left(\bar{x}^{(n)}\right)\right\}$ for the curvature, a discrete approximation to a $C^{2}$-smooth curve. Still, the sets are not yet ordered, but an ordering that corresponds to a positive orientation can be obtained as follows. Picking an arbitrary point $\bar{x}^{(k)}$, we obtain the corresponding grid cell through which the 0-level set is running. We know that it leaves the grid cell through two of its sides and choose the one that is roughly obtained by turning the corresponding normal $\nu\left(\bar{x}^{(k)}\right)$ counter-clockwise by 90 degrees. Once the next grid cell has been determined we can add the point $\bar{x}^{(j)}$ from that grid cell to the ordered set of points. Now furthermore, the next grid to be considered is uniquely defined since the 0-level set does not fork. Iterating this procedure, we obtain an ordering of the boundary points that corresponds to a positive orientation.

If we arrive at the point $\bar{x}^{(k)}$ chosen in the first place before all other points have been used, this indicates that the 0-level set has several connected components. We then pick again an arbitrary point of the not yet ordered points and proceed as above. In this way we also immediately gain the information about the number of connected components of which the 0-level set consists.

\section{How to do the re-distancing?}

Let us begin the discussion with an explanation for the need of re-distancing by considering the following example. 
Example 4.19. Consider the Initial Value Problem 4.16 with initial conditions given by $C_{0,0}(\phi)=\left\{x \in \mathbb{R}^{2}:|x|=3\right\}$ and the normal velocity field

$$
F_{n}(r, \theta, t):=2(r-(3+2 t)) \sin (4 \theta)+2
$$

in polar coordinates. Then the 0-level set of the solution $\phi$ to Problem 4.16 is an expanding circle of radius $(3+2 t)$ given that $\phi$ initially satisfies $\mid$ grad $\phi \mid=1$ on $C_{0,0}$. From the level set equation (4.39) we see that $\phi$ satisfies

$$
\left.\frac{\partial \phi}{\partial t}\right|_{t=0}=-2|\operatorname{grad} \phi|
$$

on $C_{0,0}$ for all angular directions. If we now suppose that the level sets of $\phi(\cdot, 0)$ are given as concentric circles, we observe that the other level sets of $\phi$ do not move with constant speed with respect to the angle $\theta$, as is obvious from (4.47). So, for example, the circle with radius 4 around the origin does not expand as a circle. Instead, we have $F_{n}\left(4, \frac{\pi}{8}, 0\right)=4$ for the angle $\theta=\frac{\pi}{8}$, while for $\theta=-\frac{\pi}{8}$ we have $F_{n}\left(4,-\frac{\pi}{8}, 0\right)=0$. Consequently, the initially circular c-level sets for $c \neq 0$ become more and more distorted as we propagate in time.

Example 4.19 displays that the gradient of $\phi$ can flatten in certain angular regions, whereas it can become rather steep in other angular sectors. This then implies that the quality of the finite difference approximations to the gradient and to other geometric quantities become less and less accurate as we propagate in time, which may lead to considerable deterioration of the entire method.

The idea of re-distancing has been invented to overcome this unfavourable behaviour of certain velocity fields. The aim is to modify the level set function $\phi$ such that the 0-level set remains unchanged, but the gradient of $\phi$ has the same magnitude almost everywhere. In other words, re-distancing seeks to restore the signed distance function property

$$
|\operatorname{grad} \phi|=1
$$

on the computational domain.

In the literature, one can mainly find two concepts for re-distancing. The first approach, often refered to as the re-initialization equation, seeks to restore the signed distance function property (4.48) by solving the auxiliary initial value problem

$$
\frac{\partial \widehat{\phi}}{\partial t}+H\left(\phi\left(\cdot, t_{0}\right)\right)(|\operatorname{grad} \widehat{\phi}(x, t)|-1)=0, \quad \widehat{\phi}(\cdot, 0)=\phi\left(\cdot, t_{0}\right)
$$

for $\widehat{\phi}$ to steady state, and use the steady state of $\widehat{\phi}$ as a new initialization for the level set function $\phi\left(\cdot, t_{0}\right)$. Here $H$ is the Heavyside function. This auxiliary 
problem resembles the level set equation with the difference that it does not move the 0-level set at all, whereas all other level sets are driven towards restoring the signed distance function property (4.48). For a closer study of re-distancing using the re-initialization equation we refer to $[54,71]$.

In this work we will employ an approach that has been proposed by Sethian in [64] under the name of Fast Marching Method (see also [66]). The key idea of this approach is to restore the signed distance function property (4.48) by interpreting the distance of a point to the 0-level set as the crossing time of a moving front, that is travelling out from the 0-level set in normal direction with unit speed. Formally, this can be framed as a boundary value problem for the eikonal equation, namely we seek a solution of

$$
|\operatorname{grad} \widehat{\phi}|=1, \quad \widehat{\phi}(x)=0 \Leftrightarrow \phi\left(x, t_{0}\right)=0 .
$$

In the numerical realization, the Fast Marching Method treats the positive side and the negative side of the 0-level set separately. It assumes that there are accurate distance values given for all grid points nearest to the 0-level set on the particular side under consideration. These grid points are tagged as 'accepted'. We then march outwards from the accepted grid points assigning 'tentative' values to all neighbouring grid points, that are not yet accepted. These grid points are tagged as 'trial points', while all other grid points are tagged as 'far away'. The tentative values are obtained from an upwind formulation for the discretization of $|\operatorname{grad} \widehat{\phi}|^{2}=1$, that can also be thought of as applications of Huygen's principle (see [66], Chapter 8). For the assignment of tentative values we have to distinguish between five different cases.

a) In the case of a 'trial point' $x_{i, j}$ having just one 'accepted' neighbour $x_{s}$ with a distance value $\phi_{s}$ this means that we solve

$$
\left(\frac{\phi_{i, j}-\phi_{s}}{h}\right)^{2}=1
$$

for $\phi_{i, j}$. From the point of view of Huygen's principle (4.49) the value $\phi_{i, j}$ is obtained as the time when a circular front with unit speed reaches $x_{i, j}$ provided it starts at $x_{s}$ at time $\phi_{s}$.

b) In the case of a 'trial point' $x_{i, j}$ having two 'accepted' neighbours such that $x_{i, j}$ lies on the connecting line, Huygen's principle tells to consider the solutions to (4.49) for each of the neighbours separately, and use the smaller one as the value for $\phi_{i, j}$.

c) In the case of a 'trial point' $x_{i, j}$ having two 'accepted' neighbours $x_{s_{1}}, x_{s_{2}}$ such that the three points are not collinear, the upwind approximation to $|\operatorname{grad} \widehat{\phi}|^{2}=1$ reads

$$
\left(\frac{\phi_{i, j}-\phi_{s_{1}}}{h}\right)^{2}+\left(\frac{\phi_{i, j}-\phi_{s_{2}}}{h}\right)^{2}=1,
$$


which again needs to be solved for $\phi_{i, j}$. As (4.50) is a quadratic equation it may have two or only one solution, or maybe even no solution at all. Although the latter two cases can be ruled out theoretically from the perspective of Huygen's principle, they may occur in practice due to poor or inconsistent initial data or numerical error. As an indicator for such a situation we may use the sign of

$$
\left(\frac{\phi_{s_{2}}-\phi_{s_{1}}}{h}\right)^{2}-1
$$

If the sign is positive, we are confronted with poor data. In this case we neglect the neighbour corresponding to the larger value of $\left\{\phi_{s_{1}}, \phi_{s_{2}}\right\}$ and proceed with the other neighbour by applying case a). In the other case, when the sign of (4.51) is non-positive, we can be sure that at least one solution to (4.50) exists. We then choose the solution of (4.50), which corresponds to the '+'-sign in the quadratic formula, as 'tentative' distance value for $\phi_{i, j}$.

d) In the case of the 'trial point' $x_{i, j}$ having three 'accepted' neighbours, these can be grouped as two times case c). Again, Huygen's principle tells to consider the solutions to the two situations separately, and use the smaller one. It turns out that it is equivalent to first apply case b) to the two neighbours being collinear with $x_{i, j}$ to find the correct side, and then apply case c) to this point and the remaining neighbour.

e) In the case of the 'trial point' $x_{i, j}$ having four 'accepted' neighbours, we proceed in the spirit of d) and apply c) to each non-collinear pair, and then take the minimal value. Equivalently, we apply b) to both collinear directions to find the correct sides, and then apply c) only once.

After all 'trial points' have been assigned according tentative values, we begin the marching process, repeating the following steps:

1. We tag one of the 'trial points' as 'accepted', namely the one that has the smallest 'tentative' value, since this 'trial point' is reached first by a front moving outward from the band of 'accepted' grid points with unit speed.

2. We then tag all neighbouring grid points of this newly 'accepted' grid point that are not yet accepted as 'trial points'. This means that some 'far away' points will become 'trial points', and some 'trial points' will get their tag renewed.

3. We (re-)calculate the 'tentative' distance values for all 'trial points', where the tag has been changed or renewed in the previous step. This can be interpreted from the point of view of Huygen's principle: The newly 'accepted' point sends out a circular wave with unit speed that contributes to all neighbouring grid points that have not yet been accepted. 
The Fast Marching Method is an upwind method in the sense of the third step, as the distance values only influence grid points with larger distance values. So the information is flowing away from the 0-level set or downwind. We illustrate the marching process in Figure 4.9.

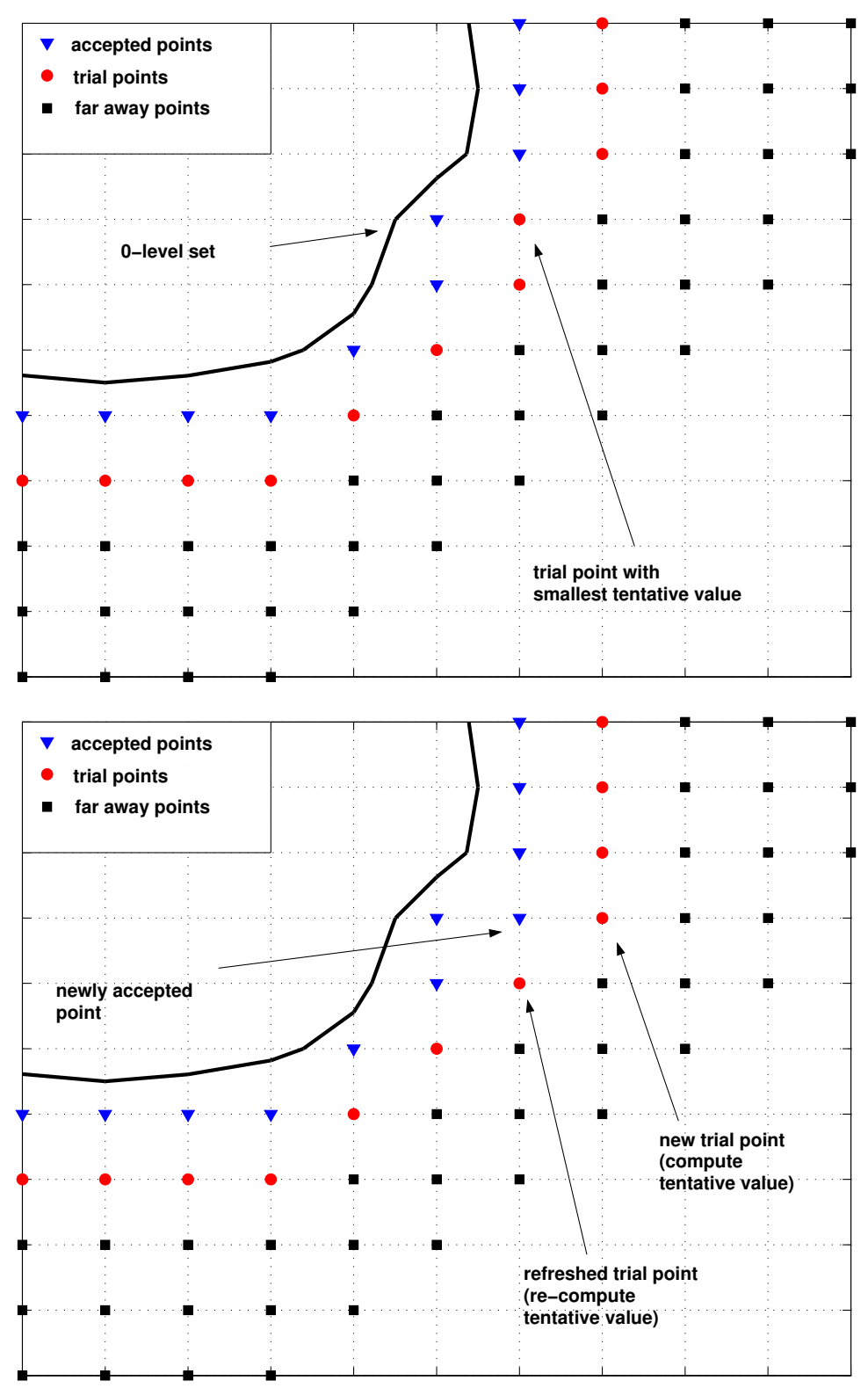

Figure 4.9: Illustration of the way the Fast Marching Method works

In the upper schematic, we see a possible situation for the Fast Marching Method with the 'accepted' grid points marked as blue triangles, the 'trial points' in as red circles, and the 'far away' grid points as black squares. Consider the 'trial point' with minimum 'tentative' distance value as indicated. In the lower schematic we 
see how the bands of 'accepted', 'far away' and 'trial points' have changed after the grid point with minimum tentative distance value has been accepted to the 'accepted' grid points. The grid point itself now has a blue triangle as tag and is indicated as newly accepted point. The neighbours that are not yet accepted get their tags re-refreshed. In the schematic the lower and right hand side neighbours are affected. The left hand side neighbour has not had a tentative distance value before as it was tagged as 'far away'. The lower neighbour has had a tentative distance value before, which we have to re-compute as it now is a 'trial point' with two accepted neighbours instead of only one as before. Note furthermore that the tentative values of all other 'trial points' remain unaffected, because the tags of their neighbours have not changed.

Proceeding in this way, we assign distance values in an ascending order to all grid points. The complexity of this algorithm if of order $\mathcal{O}(n \log n)$, where $n$ is the total number of grid points. Note that the $\log n$ term is due to the fact that after each accepting of a grid point, we have to find the 'trial point' with minimal tentative value, which involves a sorting algorithm that has complexity $\mathcal{O}(n \log n)$. In [54] Osher and Fedkiw suggest to exploit a heapsort algorithm as used by Tsitsiklis in [73], but we have found the implemented min-algorithms of standard software packages to be much faster than $C$-implementations of heapsort.

Furthermore, we observe that the Fast Marching Method and the narrow band technique described on page 128 complement each other ideally. On the one hand the narrow band technique reduces the overall complexity of the Fast Marching Method as it reduces the number of points to which we have to assign a distance value. On the other hand the desired bandwidth for the narrow band technique is easily realized in the framework of the Fast Marching Method. Namely, we simply stop tagging 'far away' points as 'trial points' once we have reached the desired bandwidth. This creates a natural stopping criterion for the Fast Marching Method.

\section{How to extend a 'boundary' velocity?}

Let us first of all discuss the need for extending a 'boundary' velocity. Although we are interested only in the 0-level set of the solution $\phi$ to Problem 4.16, we see from the level set equation (4.39) that in the initial value formulation the normal component $V_{n}$ of the velocity field has to be known on all of $\mathbb{R}^{2}$, i.e. for all level sets. Contrary to the examples in the paper of Sethian and Osher [56] or the examples for curvature driven motion (see [54], Chapter 4), where the geometry of the other level sets naturally defines a velocity field everywhere, there are many situations such as the Geometric Optimization Problem from Chapter 3, where there exist no natural speed functions off the 0-level set. As such a speed function is needed to make the level set algorithm work, it has to be somehow obtained from the well-defined 'boundary' velocity. 
The issue of creating an extension velocity has been around in several applications of level set methods (see for example [3, 15, 67, 71, 77]), although none of these schemes seems to have proved superior against the others. It has been common to all these approaches that explicit formulations for the 'boundary' velocity were proposed, for which it made sense to evaluate them not only on the 0-level set itself, but also in a small neighbourhood of it. Thereby these approaches already solved the most immediate difficulty of creating a meaningful velocity somewhere off the 0-level set. In a second step, the values for the velocity in this small neighbourhood were extended in an appropriate fashion to all 0-level set. In our description we will follow the approach of Adalsteinsson and Sethian [3] for the second step, as their approach can be easily incorporated into the re-distancing algorithm. For the first step we will present a newly developed approach that carries the idea of [3] one step further.

Let us therefore illustrate the idea of [3] in more detail. Suppose that we are given a level set function $\phi$, which is a signed distance function, i.e. $\phi$ satisfies the signed distance function property (4.48). Furthermore we are given a 'boundary' velocity $F_{n}$, which we interpret as the restriction of the normal component $V_{n}$ of a velocity field to the 0 -level set via

$$
\left.V_{n}\right|_{C_{t, 0}(\phi)}=F_{n}
$$

We immediately see that the requirement (4.52) for $V_{n}$ leaves considerable amount of freedom to design a velocity with desirable features. Adalsteinsson and Sethian raise the natural claim that the first property an extension velocity should have, is to retain the properties of the level set function $\phi$, namely the signed distance function property (4.48). As a sufficient condition they required the extended velocity to be constant perpendicular to the level lines of $\phi$, i.e. the extended velocity $V_{n}$ has to satisfy

$$
\left\langle\operatorname{grad} V_{n}, \operatorname{grad} \phi\right\rangle=0 .
$$

That (4.53) is indeed a necessary and sufficient condition can be seen from the following simple calculation assuming that $V_{n}$ and $\phi$ are smooth. Then it holds that

$$
\begin{aligned}
\frac{\partial}{\partial t}|\operatorname{grad} \phi|^{2} & =2\left\langle\operatorname{grad} \phi, \operatorname{grad} \frac{\partial \phi}{\partial t}\right\rangle \\
& =-2\left\langle\operatorname{grad} \phi, \operatorname{grad}\left(V_{n}|\operatorname{grad} \phi|\right)\right\rangle \\
& =-2\left\langle\operatorname{grad} \phi, \operatorname{grad} V_{n}\right\rangle,
\end{aligned}
$$

where we have used the level set equation (4.39) and the signed distance function property (4.48) of $\phi$ at time $t_{0}$.

Algorithmically, the creation of an extension velocity satisfying (4.53) can be easily incorporated into the Fast Marching Method described in the paragraph on 
re-distancing. Assume that we have finished the initial step of the Fast Marching Method and assigned 'tentative' distance values to all 'trial points' for the initial band of accepted grid points. Assume furthermore that we have values for $V_{n}$ on the accepted grid points. (This is the numerical eaquivalent to the widely used assumption from the literature that the 'boundary' velocity can be evaluated in a small neighbourhood of the 0-level set.)

As we tag the 'trial point' $x_{i, j}$ with minimal 'tentative' distance value $\phi_{i, j}$ as 'accepted', we know that the distance value is obtained either from the knowledge of a single neighbouring grid point $x_{s}$, or from the knowledge of two neighbouring grid points $x_{s_{1}}$ and $x_{s_{2}}$ which are not collinear as a triplet $\left(x_{i, j}, x_{s_{1}}, s_{s_{2}}\right)$. For the first case we may assume without loss of generality that $x_{i, j}$ and $x_{s}$ coincide in their second component. Then the approximation to the gradient of $\phi$ vanishes in the second component so that the discrete upwind analog of (4.53) is given by

$$
\left\langle\left(\frac{\phi_{i, j}-\phi_{s}}{h}, 0\right),\left(\frac{V_{i, j}-V_{s}}{h}, \frac{V_{i, j}-V^{*}}{h}\right)\right\rangle=0,
$$

which can be solved for $V_{i, j}$ yielding

$$
V_{i, j}=V_{s}
$$

We note in particular, that we only use the known value $V_{s}$ for the construction of $V_{i, j}$, while the unknown value $V^{*}$ is neglected due to our assumption on the gradient direction for $\phi$. For the second case we similarly assume without loss of generality that $x_{i, j}$ and $x_{s_{1}}$ coincide in their second component, while $x_{i, j}$ and $x_{s_{2}}$ coincide in their first component. Then both influence the gradient direction for $\phi$ and the upwind discretization of (4.53) reads

$$
\left\langle\left(\frac{\phi_{i, j}-\phi_{s_{1}}}{h}, \frac{\phi_{i, j}-\phi_{s_{2}}}{h}\right),\left(\frac{V_{i, j}-V_{s_{1}}}{h}, \frac{V_{i, j}-V_{s_{2}}}{h}\right)\right\rangle=0,
$$

which is again solved for $V_{i, j}$ to find

$$
V_{i, j}=\frac{V_{s_{1}}\left(\phi_{i, j}-\phi_{s_{1}}\right)+V_{s_{2}}\left(\phi_{i, j}-\phi_{s_{2}}\right)}{\left(\phi_{i, j}-\phi_{s_{1}}\right)+\left(\phi_{i, j}-\phi_{s_{2}}\right)} .
$$

In this case both values $V_{s_{1}}$ and $V_{s_{2}}$ that contribute to $V_{i, j}$ are known, since they belong to grid points with an 'accepted' distance value.

As all other cases occuring in the algorithm of the Fast Marching Method can be described as the combination of a preliminary step and one of the two cases above, (4.54) and (4.55) give a complete algorithm for the creation of an extension velocity satisfying a discrete form of (4.53) that is easily incorporated into the Fast Marching Method provided we have values for $V_{n}$ at the grid points 'closest' to the 0-level set. 
As mentioned before, the existing approaches in the literature assume that the values for $V_{n}$ at the grid points 'closest' to the 0 -level set can be obtained from some analytical expression. In our approach we differ from this widely used assumption, and try to follow a concept which is in a certain sense the dual formulation to the contouring algorithm described in one of the the previous paragraphs. In the contouring algorithm we have considered a grid cell, through which the 0-level set is running, and extrapolated the position of a point on the 0-level set from the known distance values on the grid points at the corner of this grid cell. The dual formulation for the extension of a 'boundary' velocity, which is assumed to be known on exactly these points on the 0-level set, consists in extrapolating these values using the information about the gradient direction on the 0-level set.

To be more specific, let us consider a grid point $x_{i, j}$ which is 'closest' to the 0-level set. Then, in at least one of its neighbouring grid points, the level set function $\phi$ has a different sign, and as the level set function is continuous, the 0-level set intersects the grid line which connects these two grid points. Thus, there are at least two grid cells adjacent to $x_{i, j}$ through which the 0-level set is running, and we know from the contouring algorithm that in each of these grid cells we have constructed a point $\bar{x}^{(k)}$ that approximates the position of the 0-level set. Furthermore, we know from the contouring algorithm that we can divide the position of the 0-level set in an indivdual grid cell into two types which are shown in Figure 4.4 a) and b). In each case we can calculate

i) the distance $d_{i, j}:=\left|\bar{x}-x_{i, j}\right|$ between the point $\bar{x}$ on the 0-level set and the grid point $x_{i, j}$ that is a corner of the grid cell. In case of Figure 4.10 a) the point $x_{i, j}$ may be any corner, in the case of Figure $4.10 \mathrm{~b}$ ) it may be one of the upper corners or the lower right corner.

ii) the signed distance $s_{i, j}$ between the corner $x_{i, j}$ and the point $\bar{x}_{\text {prop }}$. The location of $\bar{x}_{\text {prop }}$ is obtained as follows. Consider the grid line $L$ through the corner $x_{i, j}$ which does not intersect with the 0 -level set on the grid cell under consideration. Then propagate $\bar{x}$ in normal direction until it reaches the grid line $L$, and define $\bar{x}_{\text {prop }}$ as the intersection point. Now $s_{i, j}$ is obtained by solving

$$
\bar{x}+t \operatorname{grad} \phi\left(\bar{x}, t_{0}\right)=x_{i, j}+s_{i, j} v_{i, j},
$$

where $v_{i, j} \in\left\{ \pm e_{1}, \pm e_{2}\right\}$ is chosen as the direction parallel to $L$ pointing from $x_{i, j}$ into the grid cell under consideration.

The above algorithm is depicted in Figure 4.10. We guarantee its soundness with the following remarks.

Remark 4.20. For the distances $d_{i, j}$ and the signed distances $s_{i, j}$ the following holds: 
1. The normal direction at $\bar{x}$ and $L$ always intersect, since the normal direction at $\bar{x}$ is given by the approximation to the gradient at $\bar{x}$. Consequently, the normal direction being parallel to $L$ would imply that the level set function $\phi$ is constant perpendicular to $L$. This is a contradiction as the 0 -level set intersects the grid line perpendicular to $L$, and hence the signs of the level set function $\phi$ at $x_{i, j}$ and at the neighbouring grid point on that grid line are different.

2. In Figure 4.10 b) we cannot determine a grid line $L$ for the lower left corner that does not intersect with the 0-level set on the grid cell. But we note that the contouring algorithm has constructed

$$
\bar{x}:=x_{i, j}-\phi^{n}\left(x_{i, j}\right) \frac{\operatorname{grad} \phi^{n}\left(x_{i, j}\right)}{\left|\operatorname{grad} \phi^{n}\left(x_{i, j}\right)\right|},
$$

which implies that (4.56) yields $s_{i, j}=0$ for any choice of $L$ and $v_{i, j}$.

a)

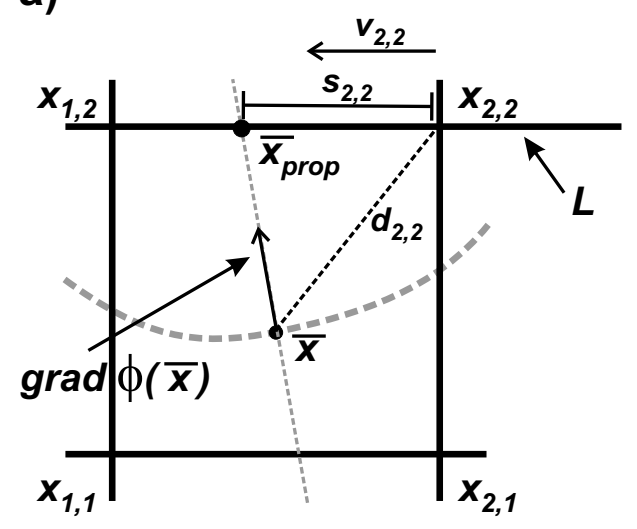

b)

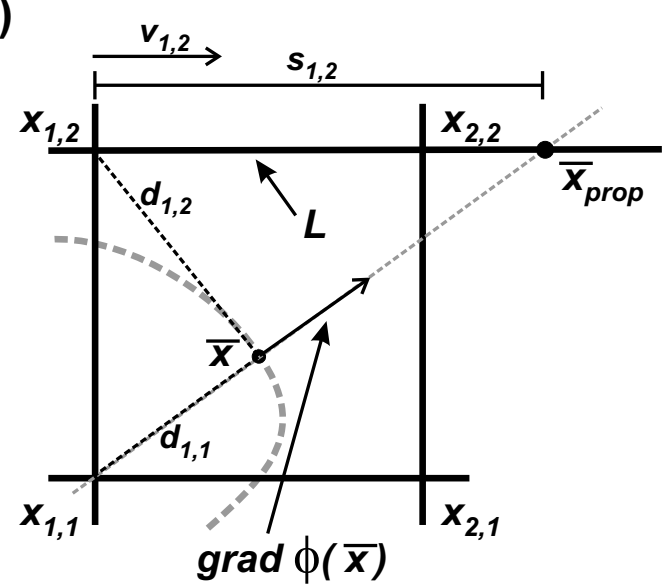

Figure 4.10: Illustration of the construction of $d_{i, j}, \bar{x}_{\text {prop }}$ and $s_{i, j}$ for the possible positions of the 0-level set in a grid cell

Proceeding in this fashion for all grid cells adjacent to $x_{i, j}$ through which the 0-level set is running, we obtain from each of these grid cells a set of values of the form $\left(d_{i, j}^{(k)}, s_{i, j}^{(k)}, f^{(k)}\right)$, where $f^{(k)}$ is the value of the 'boundary' velocity at $\bar{x}^{(k)}$ and the upper index $(k)$ denotes the dependence on the grid cell. Then the value of the extension velocity $V_{n}$ at $x_{i, j}$ is obtained as follows.

In the case $\left\{s_{i, j}^{(k)} \leq 0\right\} \neq \emptyset$ we define

$$
V_{n}\left(x_{i, j}\right):=f^{\left(k_{0}\right)}, \quad \text { where } \quad\left|\frac{d_{i, j}^{\left(k_{0}\right)}}{f^{\left(k_{0}\right)}}\right|=\min \left\{\left|\frac{d_{i, j}^{(k)}}{f^{(k)}}\right|: s_{i, j}^{(k)} \leq 0\right\},
$$


and in the case $\left\{s_{i, j}^{(k)} \leq 0\right\}=\emptyset$ we define

$$
V_{n}\left(x_{i, j}\right):=\frac{s_{i, j}^{\left(k_{2}\right)} f^{\left(k_{1}\right)}+s_{i, j}^{\left(k_{1}\right)} f^{\left(k_{2}\right)}}{s_{i, j}^{\left(k_{1}\right)}+s_{i, j}^{\left(k_{2}\right)}},
$$

where the grid cells $k_{1}, k_{2}$ are chosen such that

- the restriction of the 0-level set to these grid cells is a connected set,

- the weighted sum

$$
q_{i, j}\left(k_{1}, k_{2}\right):=\frac{s_{i, j}^{\left(k_{2}\right)}}{s_{i, j}^{\left(k_{1}\right)}+s_{i, j}^{\left(k_{2}\right)}}\left|\frac{d_{i, j}^{\left(k_{1}\right)}}{f^{\left(k_{1}\right)}}\right|+\frac{s_{i, j}^{\left(k_{1}\right)}}{s_{i, j}^{\left(k_{1}\right)}+s_{i, j}^{\left(k_{2}\right)}}\left|\frac{d_{i, j}^{\left(k_{2}\right)}}{f^{\left(k_{2}\right)}}\right|
$$

of the arrival times is minimal over all pairs of grid cells satisfying the first property.

In words, the above way of defining an extension velocity on the grid points 'closest' to the 0-level set works as follows.

If there are points $\bar{x}^{(k)}$ on the 0 -level set in grid cells adjacent to $x_{i, j}$, that would hit $x_{i, j}$ directly when propagated in normal direction, we determine the one which would reach $x_{i, j}$ first and extend the according value of the 'boundary' velocity the grid point $x_{i, j}$. (Note that in (4.57) we are minimizing the quotient of distance and speed, i.e. the time when the 0-level set moving from $\bar{x}^{(k)}$ with speed $f^{(k)}$ in the direction of $x_{i, j}$ would reach $x_{i, j}$.) This case occurs whenever $x_{i, j}$ is the lower left corner of (a maybe rotated version of) Figure $4.10 \mathrm{~b}$ ).

For all the other corners in Figure 4.10 the propagation of $\bar{x}^{(k)}$ in normal direction does not necessarily lead to hitting $x_{i, j}$, but we see that it might happen in the case of Figure 4.10 a) that $\bar{x}_{\text {prop }}^{(k)}$ lies on the other side of $x_{i, j}$, i.e. that the signed distance $s_{i, j}^{(k)}$ is negative. In that case there has been an intermediate time where the position of the 0-level set is of the form of Figure $4.10 \mathrm{~b}$ ). Hence, we assume in this case that $f^{(k)}$ is the only value of the 'boundary' velocity that influences the extension velocity $V_{n}\left(x_{i, j}\right)$ from this grid cell. This is why the case $s_{i, j}^{(k)}<0$ is subsumed under (4.57).

The remaining case to consider is that a point $\bar{x}^{(k)}$ on the 0-level set does not hit $x_{i, j}$ directly, and the propagated point $\bar{x}_{\text {prop }}^{(k)}$ on the same grid line of $x_{i, j}$ has a positive signed distance $s_{i, j}^{(k)}$ to $x_{i, j}$. Then the reasoning is as follows. The further apart $\bar{x}_{\text {prop }}^{(k)}$ and $x_{i, j}$ are, the less influence $f^{(k)}$ should have on $V_{n}\left(x_{i, j}\right)$, and the closer they are, the more influence $f^{(k)}$ should have. Assume that we have one set of values $\left(d_{i, j}^{\left(k_{1}\right)}, s_{i, j}^{\left(k_{1}\right)}, f^{\left(k_{1}\right)}\right)$ for $x_{i, j}$. As we have argued before, the 0 -level set leaves the grid cell through one grid line on which $x_{i, j}$ lies. Hence, the 0-level set also goes through the neighbouring grid cell and provides a second 
set of values $\left(d_{i, j}^{\left(k_{2}\right)}, s_{i, j}^{\left(k_{2}\right)}, f^{\left(k_{2}\right)}\right)$ for $x_{i, j}$ there. Now both $f^{\left(k_{1}\right)}$ and $f^{\left(k_{2}\right)}$ influence the extension velocity $V_{n}\left(x_{i, j}\right)$. Hence, we extend the 'boundary' velocity using a convex combination of $f^{\left(k_{1}\right)}$ and $f^{\left(k_{2}\right)}$ that is inversely proportional to the distances between the grid point $x_{i, j}$ and the propagated boundary points $\bar{x}_{\text {prop }}^{(k)}$, i.e. $f^{\left(k_{1}\right)}$ is weighted stronger than $f^{\left(k_{2}\right)}$ in (4.58) if $s_{i, j}^{\left(k_{1}\right)}<s_{i, j}^{\left(k_{2}\right)}$, and vice versa. Since the configuration described above might occur several times for $x_{i, j}$ (at most once for each direction), we have to decide in the case of multiple occurences which convex combination to take. As a criterion we use the same convex combination for the arrival times that has been used for the values of the 'boundary' velocities, following the idea that the point with the smaller value for the signed distance should get a stronger weight, and vice versa.

We illustrate the way the algorithm works in two examples in Figure 4.11. In Figure $4.11 \mathrm{a}$ ) there are two grid cells with boundary points $\bar{x}^{\left(k_{1}\right)}$ and $\bar{x}^{\left(k_{2}\right)}$ such that $x_{i, j}$ is exactly in normal direction. Hence, the value for the extension velocity is chosen using (4.57). In Figure $4.11 \mathrm{~b}$ ) for none of the boundary points $x_{i, j}$ is in normal direction. Hence, we choose the pair of boundary points $\bar{x}^{\left(k_{1}\right)}$ and $\bar{x}^{\left(k_{2}\right)}$, lying on the same connected part of the 0-level set, for which the sum of the weighted distances is smallest. Then the value for the extension velocity is obtained by a convex combination of $f^{\left(k_{1}\right)}$ and $f^{\left(k_{1}\right)}$ that is inversely proportional to the distance between $x_{i, j}$ and the propagated points $\bar{x}_{\text {prop }}^{\left(k_{1}\right)}$ and $\bar{x}_{\text {prop }}^{\left(k_{2}\right)}$ using (4.58) for the corresponding choice of $k_{1}$ and $k_{2}$.

\section{How to propagate a level set function in time?}

The time-stepping algorithm is the best understood algorithm in level set methods, and their strongest point. Various schemes have been proposed yielding different temporal and spatial accuracy. For an overview we refer to the book by Osher and Fedkiw [54]. The common characteristic of all proposed schemes is the particular perspective that is taken for the discretization of the spatial gradient, which is taken from hyperbolic conservation laws. We will illustrate this idea in the following example.

Example 4.21. Consider the one-dimensional wave equation

$$
u_{t}(x, t)+u_{x}(x, t)=0, \quad u(x, 0)=f(x),
$$

which has the exact solution $u(x, t)=f(x-t)$. This means that the characteristics for $u$ are lines of slope 1 . In other words, information is travelling from lower values of $x$ to higher values of $x$.

If we are going to discretize (4.59), we have to think of schemes that approximate this behaviour most adequately. Using Taylor expansion in the time-variable we find

$$
u_{t}(x, t)=\frac{u(x, t+\Delta t)-u(x, t)}{\Delta t}+\mathcal{O}\left((\Delta t)^{2}\right)
$$


a)

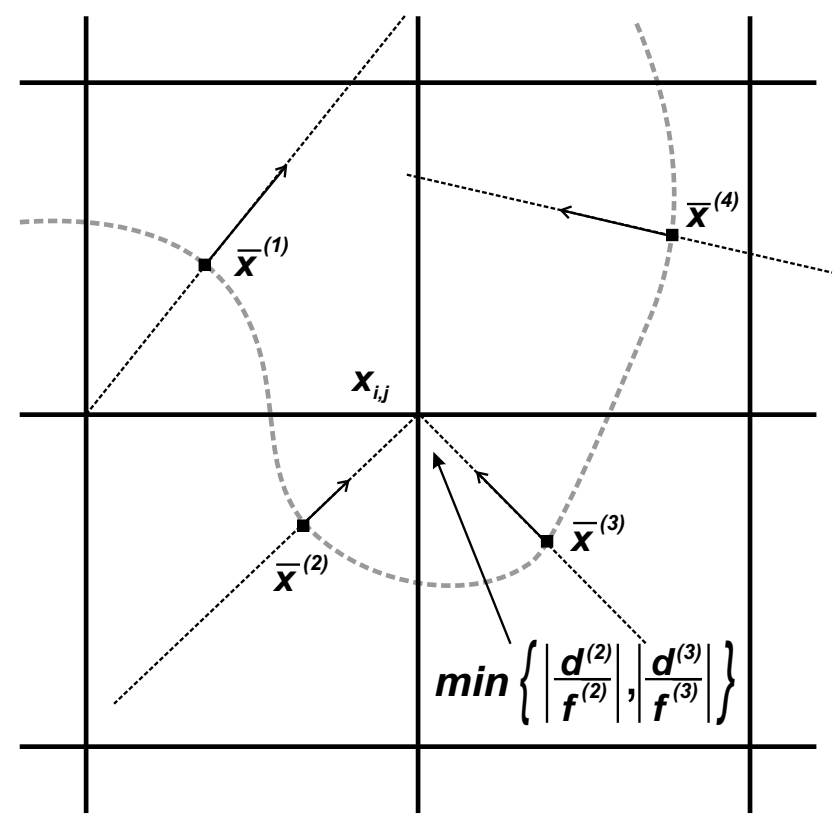

b)

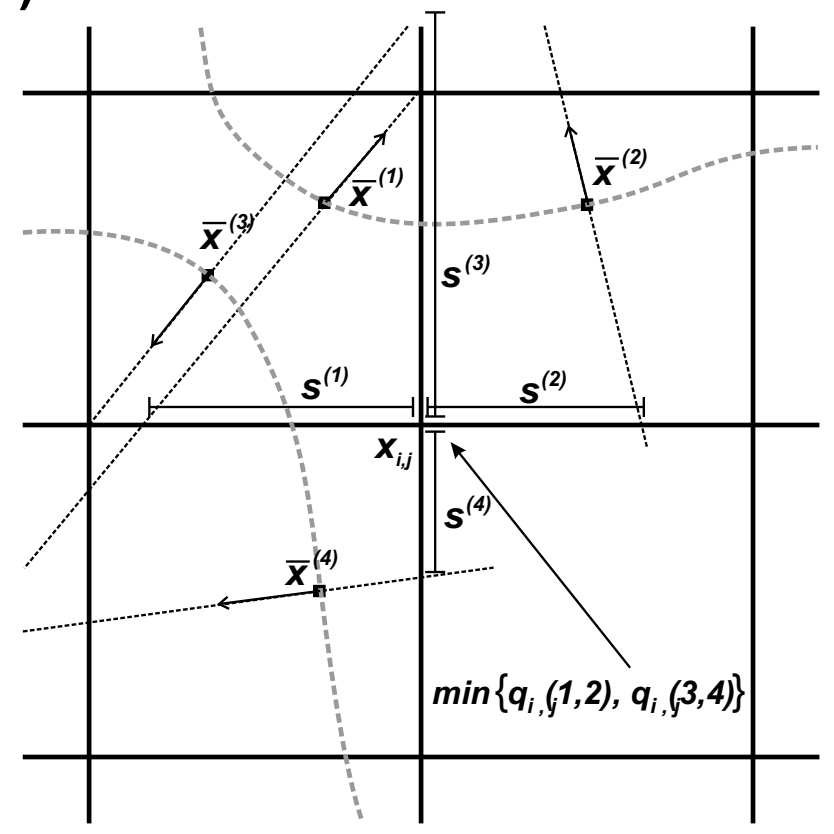

Figure 4.11: Extension of a 'boundary' velocity to the grid points closest to the 0-level set - two examples 
Similarly, for the discretization of the spatial derivative we may choose

$$
\begin{aligned}
D_{x}^{-} u & :=\frac{u(x, t)-u(x-h, t)}{h}, \\
D_{x}^{+} u & :=\frac{u(x+h, t)-u(x, t)}{h}, \\
D_{x}^{0} u & :=\frac{u(x+h, t)-u(x-h, t)}{2 h} .
\end{aligned}
$$

Although $D_{x}^{0} u$ is the most accurate approximation to $u_{x}(x, t)$, the approach via the characteristics suggests to use $D_{x}^{-} u$ instead, as $u(x, t+\Delta t)$ is then built from the information of $u(x, t)$ and $u(x-h, t)$, i.e the information information is roughly flowing in the direction of the characteristics, whereas choosing $D_{x}^{+} u$ or $D_{x}^{0} u$ would cause the information to flow numerically in a direction almost perpendicular to the characteristics.

Example 4.21 shows that in a hyperbolic system the approximation to the spatial gradient has to depend on the direction in which the information is propagating. In view of the level set equation (4.39) this means that the approximation of the spatial gradient has to be chosen depending on the sign of $V_{n}$.

For our work we have employed the most simple forms of discretization for both the temporal and the spatial variables of the level set equation (4.39), namely first order accurate explicit forward Euler time discretization

$$
\frac{\partial \phi(x, t)}{\partial t} \approx \frac{\phi(x, t+\Delta t)-\phi(x, t)}{\Delta t}
$$

as well as first order accurate upwind discretization for the spatial gradient according to Godunov's scheme, which we apply using the formulae of Rouy and Tourin [61]:

$$
\left(\frac{\partial \phi(x, t)}{\partial x_{i}}\right)^{2} \approx \max \left(\max \left(D_{x, i}^{-} \phi, 0\right)^{2}, \min \left(D_{x, i}^{+} \phi, 0\right)^{2}\right)
$$

if $V_{n}(x, t)>0$, and

$$
\left(\frac{\partial \phi(x, t)}{\partial x_{i}}\right)^{2} \approx \max \left(\min \left(D_{x, i}^{-} \phi, 0\right)^{2}, \max \left(D_{x, i}^{+} \phi, 0\right)^{2}\right)
$$

if $V_{n}(x, t)<0$. Here we have set

$$
D_{x, i}^{-} \phi:=\frac{\phi(x, t)-\phi\left(x-h e_{i}, t\right)}{h}, \quad D_{x, i}^{+} \phi:=\frac{\phi\left(x+h e_{i}, t\right)-\phi(x, t)}{h}
$$

for the standard unit vectors $e_{1}$ and $e_{2}$. This finally leads to the update

$$
\phi(x, t+\Delta t):=\phi(x, t)-\Delta t F_{n}(x, t) \sqrt{\left(\frac{\partial \phi(x, t)}{\partial x_{1}}\right)^{2}+\left(\frac{\partial \phi(x, t)}{\partial x_{i}}\right)^{2}},
$$


which can be easily implemented.

Let us finish this paragraph with a remark on the choice of the time-step. The time-step $\Delta t$ cannot be chosen arbitrarily in order to yield a convergent method. The general theory on finite difference approximations to linear partial differential equations states that a scheme is convergent if and only if it is consistent and stable, where the stability requirement guarantees that approximation errors are not amplified as we propagate in time.

Stability can be enforced using the Courant-Friedrichs-Levy condition, which says that the numerical wave should propagate as fast as the physical one. This leads to the time-step restriction

$$
\Delta t<\frac{h}{\left\|V_{n}\right\|_{\infty}}
$$

where $h$ is the mesh size for the spatial discretization. In practice the choice

$$
\Delta t=0.9 \frac{h}{\left\|V_{n}\right\|_{\infty}}
$$

has proved very successful. In view of level set methods the Courant-FriedrichsLevy condition can also be interpreted in a second way. It states basically that in one time-step the 0-level set should nowhere propagate more than one grid cell. 


\section{Chapter 5}

\section{Numerical examples}

In this chapter we present numerical results that confirm the feasibility of the algorithms proposed in Chapter 4 for both the Boundary Value Problem 2.1 and the Geometric Optimization Problem 3.3.

\subsection{The Boundary Value Problem}

In this section we are going to discuss the feasibility of the constructive approximation algorithm for the solution $u$ of the Boundary Value Problem 2.1. We will provide evidence that with our implementation of the algorithm presented in Section 4.1 we can indeed obtain exponential convergence rates in the case of $\Gamma$ and $\partial D$ being analytic.

For an exemplary geometry we will furthermore present illustrations of the distribution of the components of the magnetic field $\mathbf{B}$ as well as the corresponding current distributions.

Let us begin with a description of the geometric and physical setup for the first of the two exemplary cases we are going to study. For applicational reasons, which we are going to present in the sequel, it will be the one which we study in detail.

Example 5.1. Let the arc $\Gamma$ be a line segment of the $x_{1}$-axis given by

$$
\Gamma:=\left\{x=\left(x_{1}, x_{2}\right) \in \mathbb{R}^{2}: x_{1} \in[-1,1], x_{2}=0\right\} .
$$

Let $D$ be a simply connected, kidney-shaped domain contained in the right halfplane with center $x_{0}=(1.4,0)^{T} \in \mathbb{R}^{2}$, the boundary of which is given by

$$
\partial D:=\left\{x=x_{0}+r(t)(\cos t, \sin t) \in \mathbb{R}^{2}: t \in[0,2 \pi)\right\},
$$

where the radial function $r$ is given by

$$
r(t):=0.5+0.35 \cos t-0.05 \cos 2 t, \quad t \in[0,2 \pi) .
$$




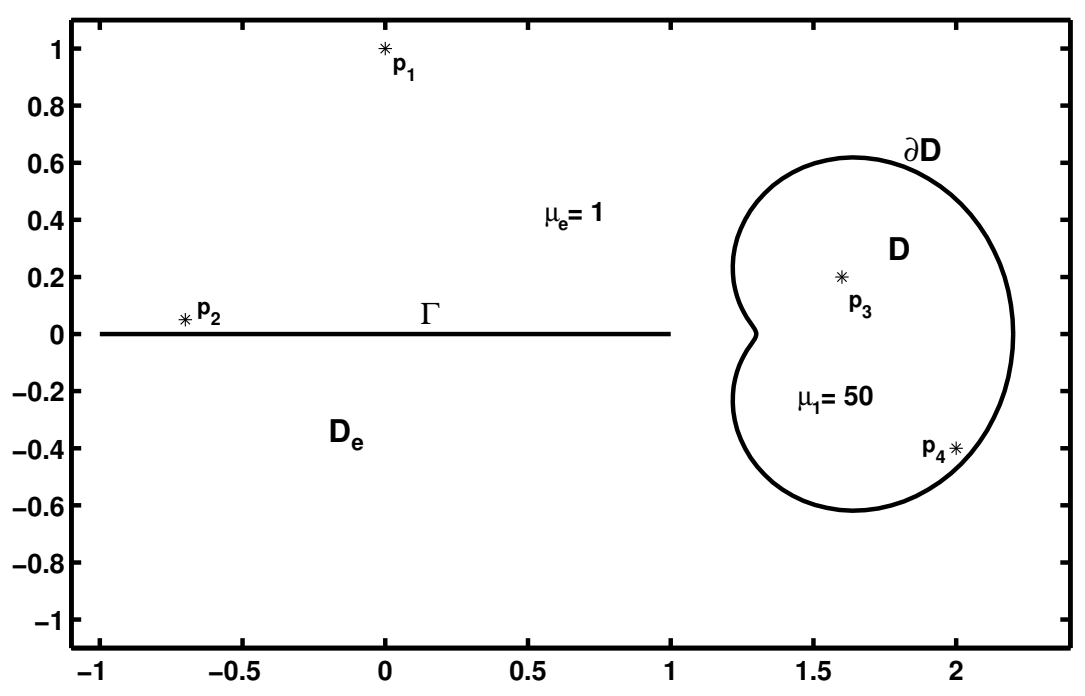

Figure 5.1: Geometric setup for Example 5.1.

Obviously, both boundaries are analytic.

The physical parameters for the magnetic permeabilities are set to $\mu_{e}=1$ and $\mu_{1}=50$, the total current is set to $I=10$. The setup is depicted in Figure 5.1.

The geometric setup is of a rather simple nature, and contains symmetries. From an applicational point of view Example 5.1 is particularly interesting. In practice, thin superconducting films are built by epitaxial chrystal growth of the superconducting material on some flat, non-conducting substrate (see also Figure 1). Hence, modelling the film by a line segment is most appropriate as it is almost the only geometry, for which we can compare the mathematical model with physical experiments. The symmetry of $D$ with respect to the line defined by $\Gamma$ is also justifiable from the invariance of the entire system with respect to translations.

Table 5.1 displays the values of the solution to Problem 2.1 for the exemplary geometry and parameters given in Example 5.1. Here, $n$ denotes the level of the discretization in the sense that we have discretized $\partial D$ using $n$ equidistanly spaced collocation points. Similarly, $\Gamma$ has been discretized using $n+1$ collocoation points that are chosen such that they are equidistantly spaced after the cosine substitution. The solution $u$ has been evaluated in the points $p_{1}, p_{2}, p_{3}$ and $p_{4}$ which are marked by $*$ in Figure 5.1. Thus, Table 5.1 reveals that we can indeed recover the theoretical result of Corollary 4.7 for the analytic geometries of Example 5.1 and obtain exponential convergence as discretization gets finer. The exponential convergence may be checked by the rule of thumb which states that the number of correct digits doubles when the discretization level doubles. If we compare the convergence behaviour for $p_{1}$ and $p_{2}$ or for $p_{3}$ and $p_{4}$, we see furthermore that the exponential convergence sets in the later the nearer the point 
is to either $\Gamma$ or $\partial D$. This is also expected from Corollary 4.7 as the constant $C$ in (4.11) depends on the distance between $x$ and $\Gamma$ or $\partial D$.

\begin{tabular}{|r|c|c|c|c|}
\hline $\mathrm{n}$ & $p_{1}=(0,1)$ & $p_{2}=(-0.7,0.05)$ & $p_{3}=(1.6,0.2)$ & $p_{4}=(2,-0.4)$ \\
\hline 8 & -1.520927088895 & -0.037599875532 & -1.898003565254 & -3.179545568397 \\
16 & -1.520351860980 & -0.108320070213 & -1.896468850137 & -3.454160875251 \\
32 & -1.520355179455 & -0.118897716268 & -1.896929014141 & 0.828562085719 \\
64 & -1.520355180674 & -0.119328288116 & -1.896929178871 & -2.268415137929 \\
128 & -1.520355180674 & -0.119328237529 & -1.896929178878 & -2.854189897583 \\
256 & -1.520355180674 & -0.119328237297 & -1.896929178878 & -2.852754451516 \\
512 & -1.520355180674 & -0.119328237297 & -1.896929178878 & -2.852754227133 \\
\hline
\end{tabular}

Table 5.1: Convergence results for Example 5.1.

From the physical point of view we are interested in the global behaviour of the potential $u$ and its partial derivatives. The reason for this has been stated in the introduction. Namely, the partial derivative with respect to $x_{1}$ can be interpreted as the negative of the $x_{2}$-component of the magnetic field $\mathbf{B}$ for the physical model and vice versa. Similarly, the $x_{1}$-component of $\mathbf{B}$ corresponds to the partial derivative with respect to $x_{2}$. The potential itself is also interesting as its level lines can be interpreted as the magnetic field lines of $\mathbf{B}$. In the Figures 5.2 - 5.4 we therefore illustrate the behaviour of the potential and its partial derivatives.

Let us make an explanatory remark for the figures first. If we take a closer look at the representation (2.10) for the potential $u$ we see that it depends on the magnetic permeability $\mu$ given by (2.1). Now $\mu$ is piecewise constant with value $\mu=1$ in the exterior and value $\mu=50$ in the interior of $D$. This induces a large amplification of the values for the partial derivatives of $u$ in the interior of $D$. For visualization purposes we have suppressed this amplification by setting $\mu \equiv 1$ in all of $\mathbb{R}^{2}$ when evaluating.

In Figure 5.2 we have displayed the distribution of the potential $u$ that solves Example 5.1. The position of the arc $\Gamma$ is marked by the solid black line, the position of $\partial D$ by the chequered areas. These areas have not been treated especially, they mark the area where the quadrature rule with which we approximate the double-layer potential yields unreliable results. For a better visualization we have also marked the isolines of the potential by the thin black lines.

If we examine Figure 5.2 a little more in detail, we see that the symmetry between the left-hand side of the arc $\Gamma$ and the right-hand side in exterior of $D$ is disturbed by $D$. The isolines of $u$ have a fixed angle at the boundary of $D$ between the inside and the outside. This angle is determined by the ratio of the magnetic permeabilities $\mu_{e}$ and $\mu_{1}$ outside and inside $D$. In comparison, the disturbance of the ellipse-shaped isolines is a little more pronounced on the side of $D$ which 


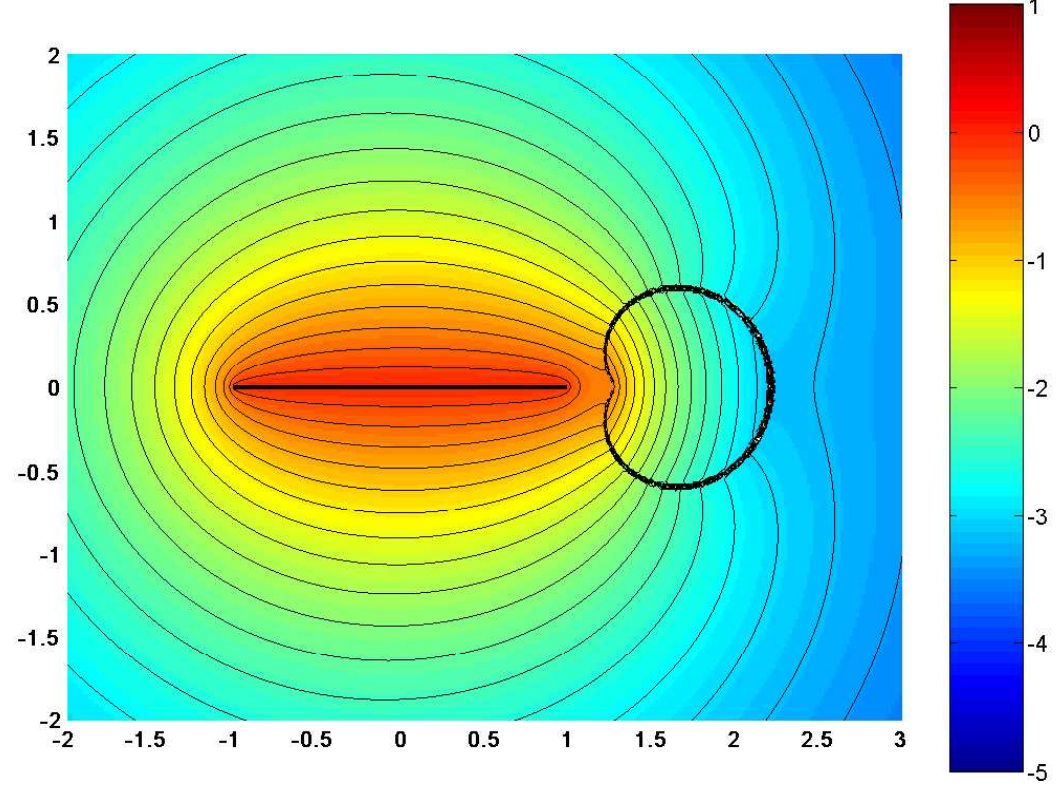

Figure 5.2: Distribution of the potential for Example 5.1 with superimposed isolines.

is not facing $\Gamma$. We observe in particular that the domain, or physically speaking the magnet, sucks in the isolines. In this sense it conditions the magnetic field surrounding the arc $\Gamma$.

Next, we consider the horizontal and vertical component of the magnetic field that are obtained from the potential according to (1). In Figure 5.3 we have depicted the horizontal component of the magnetic field $\mathbf{B}$ which correspondes to $\frac{\partial u}{\partial x_{2}}$. It already gives an impression how the jump $\left[\frac{\partial u}{\partial \nu_{\Gamma}}\right]$ of the normal derivative of $u$ across $\Gamma$ looks like. We see in particular that on the left-hand tip of $\Gamma$ a little blue area is visible on the upper side, whereas there is a corresponding little red area on the lower side of the tip. This indicates that $\left[\frac{\partial u}{\partial \nu_{\Gamma}}\right]$ becomes large in a neighbourhood of the endpoint of $\Gamma$. On the right-hand tip of $\Gamma$ this behaviour is similar. Nevertheless the stronger influence of $D$ on that side of $\Gamma$ makes the effect less visible. We remind again that the color coding for the strength of the magnetic field is not correct in $D$. The correct values are amplifications of the depicted values by a factor of 50 .

Figure 5.4 analogously shows the vertical component of the magnetic field $\mathbf{B}$ which corresponds to $-\frac{\partial u}{\partial x_{1}}$. As in the case of Figure 5.3 we note that the magnetic field has a local maximum at the tips of $\Gamma$. On the left-hand side of $\Gamma$ this peak is clearly visible in reddish colours, whereas it does not show up as clearly on the right-hand tip due to the influence of $D$.

From the representation (2.40) for the current distribution on $\Gamma$ and from the 


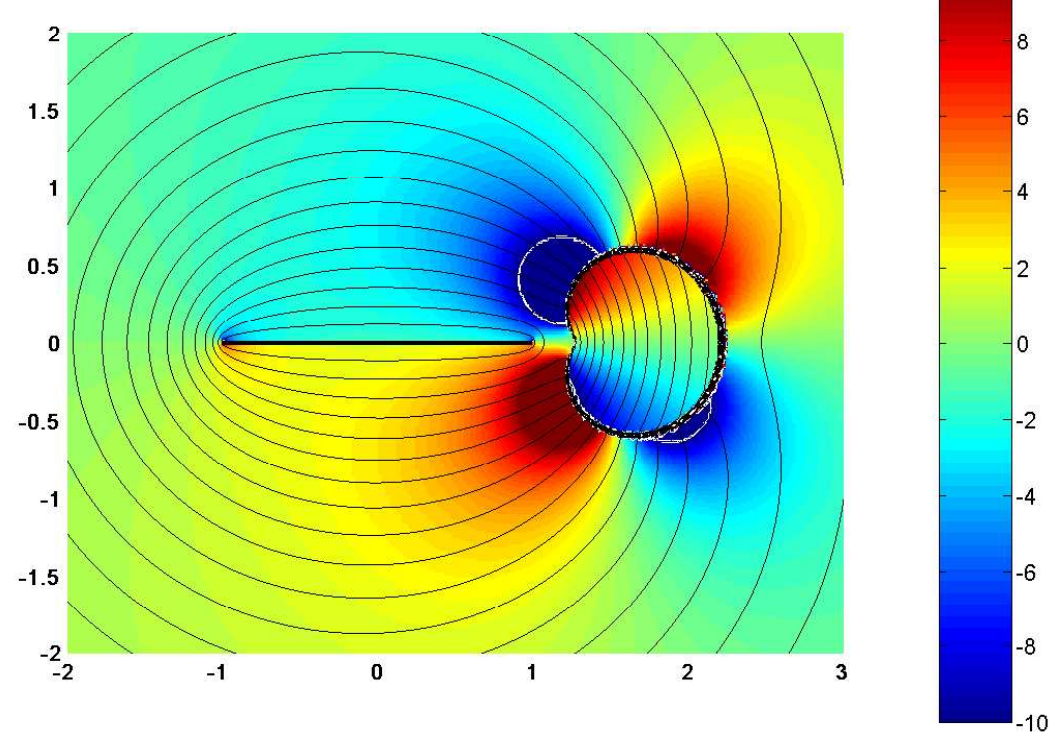

Figure 5.3: Distribution of the horizontal component of the magnetic field for Example 5.1 with superimposed field lines.

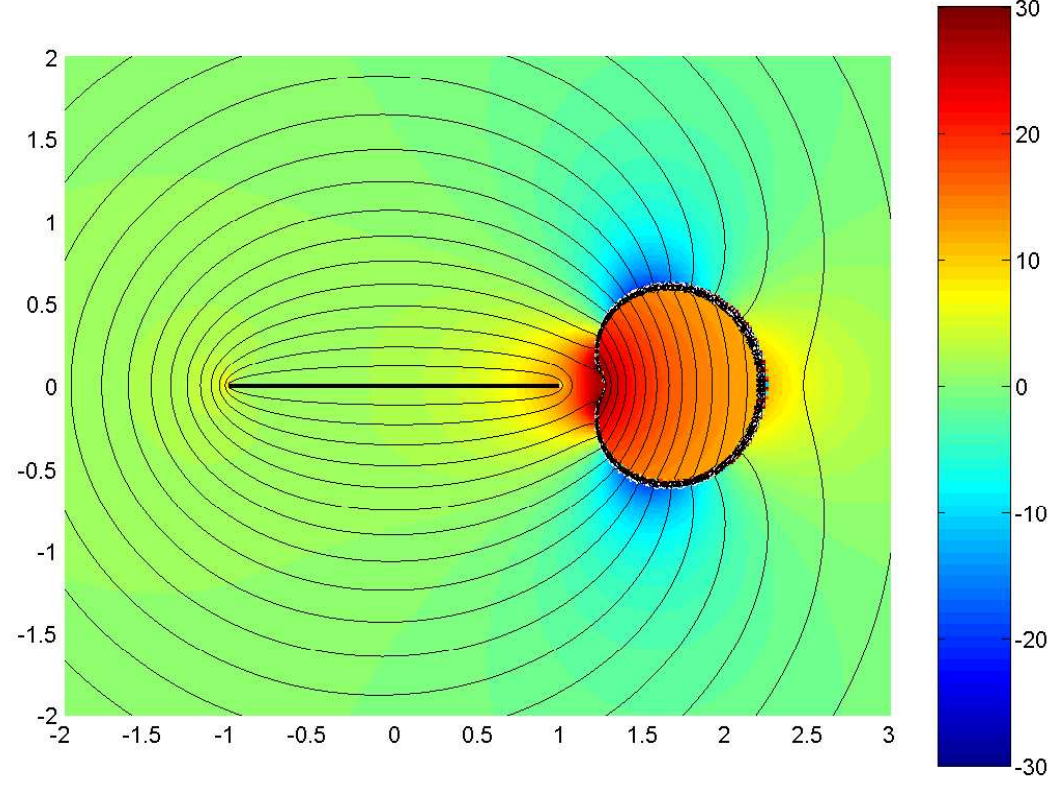

Figure 5.4: Distribution of the vertical component of the magnetic field for Example 5.1 with superimposed field lines. 
relation (2.24) between the density $\varphi \in C^{*}(\Gamma)$ that occurs in the right-hand side of (2.40) and the corresponding component of the solution of (2.36), we can express the current distribution in terms of this particular component. This gives excellent means to obtain a numerical approximation to the current distribution on $\Gamma$. We depict the current distribution for the situation of Example 5.1 in the left plot of Figure 5.5. Here, the values on the horizontal axis are given as the $x_{1}$-coordinate of the corresponding point on $\Gamma$. Due to the possible singularity of the current distribution, it is only displayed for $x \in[-1+\delta, 1-\delta]$, where $\delta$ is given as the distance from the endpoints $z_{ \pm 1}$ to the next collocation point on $\Gamma$. In Figure 5.5 we have $\delta \approx 10^{-4}$. In the right plot of Figure 5.5 the corresponding density $\widetilde{\varphi}$ that is the first component of the solution to (2.36) is displayed.
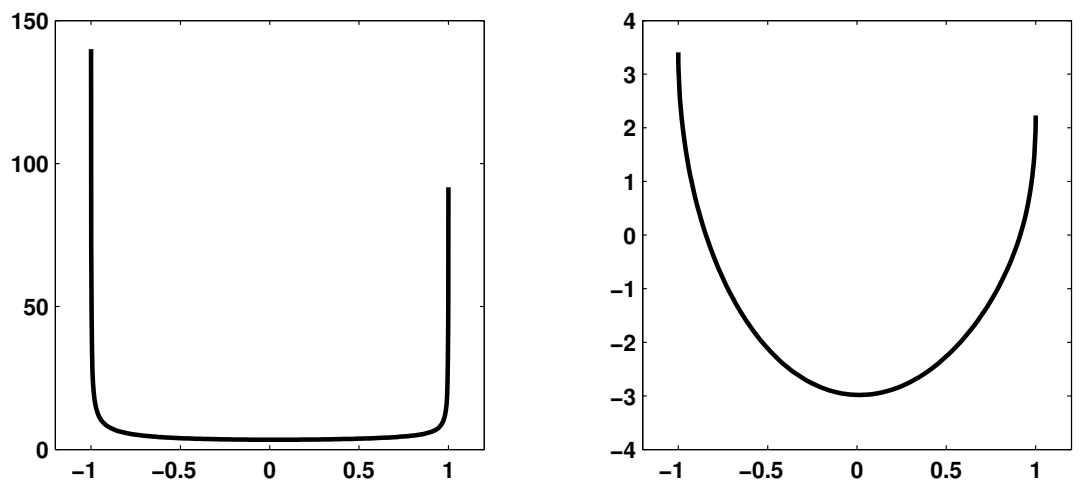

Figure 5.5: Current distribution on $\Gamma$ for Example 5.1 (left), and corresponding component $\widetilde{\varphi}$ of the solution $\Psi$ to (2.36) (right).

In the plot on the right-hand side of Figure 5.5 we see in particular that $\widetilde{\varphi}$ does not vanish at the endpoints. In view of Lemma 1.15 and Theorem 2.15 this gives evidence that the current distribution depicted on the left indeed develops singularities of square root type at the endpoints of $\Gamma$.

If we study the plot on the left-hand side of Figure 5.5 in more detail with the perspective of Chapter 3, we see that the current distribution on $\Gamma$ is not only inhomogeneous, but it is in particular assymmetric. The peak for $x=1$, which corresponds to the endpoint $z_{+1}$, is suppressed in comparison to the other peak at $x=-1$ which corresponds to $z_{-1}$. Keeping in mind that both peaks are singularities of square root type in theory, and taking into account that the mathematical model does not agree with the physical reality in a small neighbourhood of the endpoints of $\Gamma$, we can conclude that a suppression of the current is taking place on the side of $\Gamma$ that is facing the magnet $D$. This implies especially that the placing of a domain $D$ in the vicinity of the arc $\Gamma$ indeed influences the current distribution towards a homogenization. Thus, the examination of the Geometric Optimization Problem is justified.

We will finish the study of numerical examples for the Boundary Value Prob- 


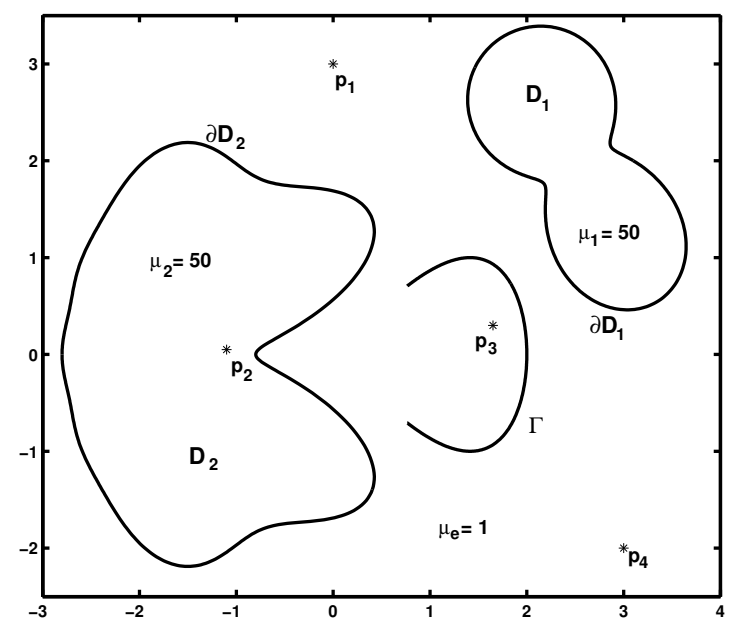

Figure 5.6: Geometric setup for Example 5.2.

lem 2.1 with a second example that displays the feasibility of the approximation scheme presented in Section 4.1 for a more involved geometric setup that contains less symmetries.

Example 5.2. Let the arc $\Gamma$ be given by the parametrization

$$
\Gamma:=\left\{\gamma(t)=\left(2 \sin \left(\frac{\pi}{2}+\frac{3 \pi t}{8}\right),-\sin \left(\pi+\frac{3 \pi t}{4}\right)\right) \in \mathbb{R}^{2}: t \in[-1,1]\right\} .
$$

Let $D$ be a domain consisting of two simply connected components. The first is a peanut-shaped component $D_{1}$ with center $x_{0,1}=(2.5,2.0)^{T} \in \mathbb{R}^{2}$, the boundary of which is given by

$$
\partial D_{1}:=\left\{x=x_{0,1}+r_{1}(t)(\cos t, \sin t) \in \mathbb{R}^{2}: t \in[0,2 \pi)\right\},
$$

where the radial function $r_{1}$ is given by

$$
r(t):=1-0.3 \cos 2 t-0.1 \cos 3 t-0.5 \sin 2 t, \quad t \in[0,2 \pi) .
$$

The second is a pacman-shaped component $D_{2}$ with center $x_{0,2}=(-1.8,0)^{T} \in \mathbb{R}^{2}$, the boundary of which is given by

$$
\partial D_{1}:=\left\{x=x_{0,2}+r_{2}(t)(\cos t, \sin t) \in \mathbb{R}^{2}: t \in[0,2 \pi)\right\},
$$

where the radial function $r_{2}$ is given by

$$
r(t):=1+1.4 h_{1,0}(t)+0.5 h_{2,0}(t)+h_{3,0}(t), \quad t \in[0,2 \pi)
$$

with the basis functions defined by (4.18). Obviously, all boundaries are analytic. The physical parameters for the magnetic permeabilities are set to $\mu_{e}=1$ and $\mu_{1}=\mu_{2}=10$, the total current is prescribed as $I=1$. The setup is depicted in Figure 5.6. 
We again choose four points, where we evaluate the potential $u$ for different levels of discretization which are displayed in the first column. As in the previous example each connected component of $D$ is discretized using $n$ equidistantly spaced collocation points. Likewise $\Gamma$ is discretized as before. The choice of the points $p_{1}, \ldots, p_{4}$ is such that we can see from Table 5.2 how the exponential convergence behaviour sets in later for $p_{2}$ and $p_{3}$ in comparison to $p_{1}$ and $p_{4}$ as we can expect the constant $C$ from (4.11) to be the larger for points that are closer to the domains of integration given by $\Gamma, \partial D_{1}$ and $\partial D_{2}$.

\begin{tabular}{|r|c|c|c|c|}
\hline $\mathrm{n}$ & $p_{1}=(0,3)$ & $p_{2}=(-1.1,0.05)$ & $p_{3}=(1.65,0.3)$ & $p_{4}=(3,-2)$ \\
\hline 8 & -0.439780111128 & -0.645583810320 & -0.003172708520 & -0.303000264934 \\
16 & -0.369829044165 & -0.884201024012 & -0.002526395722 & -0.264781301013 \\
32 & -0.333334297242 & -0.150394350861 & -0.003494080618 & -0.248763690015 \\
64 & -0.334334954889 & -0.204989978041 & -0.003408895744 & -0.249240200276 \\
128 & -0.334399699500 & -0.206730271847 & -0.003403852331 & -0.249275240634 \\
256 & -0.334400007987 & -0.206738098709 & -0.003403831087 & -0.249275407531 \\
512 & -0.334400007995 & -0.206738098991 & -0.003403831086 & -0.249275407535 \\
\hline
\end{tabular}

Table 5.2: Convergence results for Example 5.2.

We therefore abstain from examining the applicational quantities of Example 5.2 in favour of a more detailed study of the behaviour of optimized geometries that are obtained by the algorithms of the following two sections.

\subsection{The Geometric Optimization Problem via the Steepest Descent Approach}

In this section we present numerical examples for the algorithm described in Section 4.2. We will thereby cover the algorithm in its final form, but also give numerical evidence for the necessity of the regularization terms $G_{c}$ and $G_{\vartheta, d}$. For computational and conceptual simplicity we will focus mainly on magnetic environments with only one connected component.

Nevertheless, the methodology works also for any finite number of components, which we will address at the end of this section. There we also compare the computed optimal shapes for the different choices of parameters and consider the physically relevant features for one particular example.

Throughout most of the examples we apply only a defensive line search strategy for instructive reasons, namely the largest step size parameter is $\alpha=1$, and this parameter is chosen whenever it leads to an admissible geometry with a positive radial function. Only if the positivity of the radial function is violated, we choose a smaller step size. 


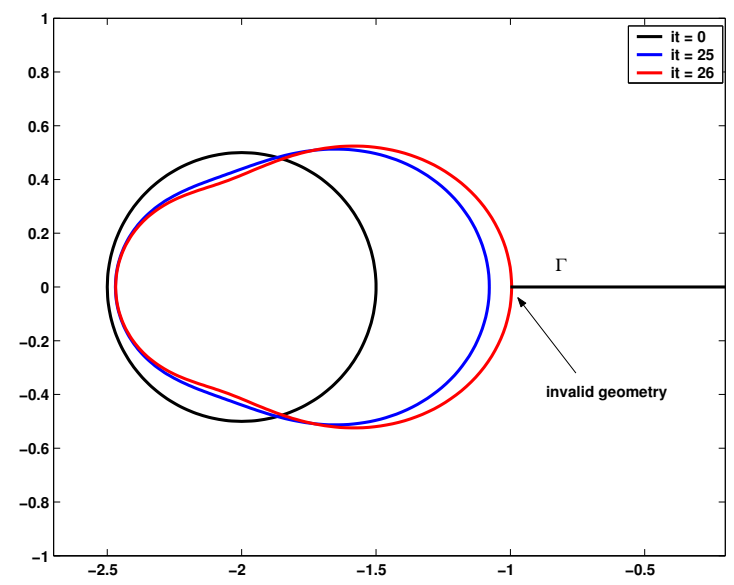

Figure 5.7: Geometric evolution of the SDA without regularization.

So, first we consider the functional without any regularization. We see from Figure 5.7 that the Steepest Descent Approach breaks down after several iterations due to an overlap of $D_{x_{0}, r}$ and $\Gamma$. At the same time the lower inset of Figure 5.8 shows that the cost functional is decreasing as the distance between $D$ and $\Gamma$ is decreasing. In particular, we see that the decrease is more pronounced if $D$ is close to $\Gamma$. From this we can already conclude that the homogenizing effect is of a local nature. This argument is supported by the current distributions that are displayed in Figure 5.8. Here we can see that the domain $D$ has almost no effect on the far side of $\Gamma$ in the sense that the current distribution remains virtually unchanged (see the large plot in Figure 5.8 in the region near $x=1$ ), even the peak of the current distribution at $x=1$ increases only marginally. The effects are different for the near side of $\Gamma$. Here, we do not only see a drastic reduction in the peak at $x=-1$, we also notice a slight decrease in the local current in the interval $[-1,-0.6]$.

Finally, in Figure 5.9 we have illustrated the evolution of the Fréchet derivative in the direction of the basis functions of $T_{3}$ (this numerical experiment has been calculated using trigonometric polynomials for the approximation space). As expected, the derivatives with respect to the sine functions vanish. This confirms the view that for the problem at hand given a symmetric configuration the algorithm should favour keeping up a symmetric configuration.

Let us finish the discussion of this experiment with two important observations. Firstly, we note from Figure 5.9 that all basis functions, for which the directional Fréchet derivatives do not vanish, also contribute to the evolution of $D$ towards $\Gamma$. This illustrates the difficulties in ensuring $D \cap \Gamma=\emptyset$ if we choose $T_{n}$ as approximation space, since the position of $\partial D$ for the angle $\vartheta=0$ is influenced by all these basis functions. Secondly, we observe from Figure 5.7 that decreasing the distance between $D$ and $\Gamma$ is the dominant feature in the minimization process. Although the approximation space has the power to form a cavity around $\Gamma$, there 


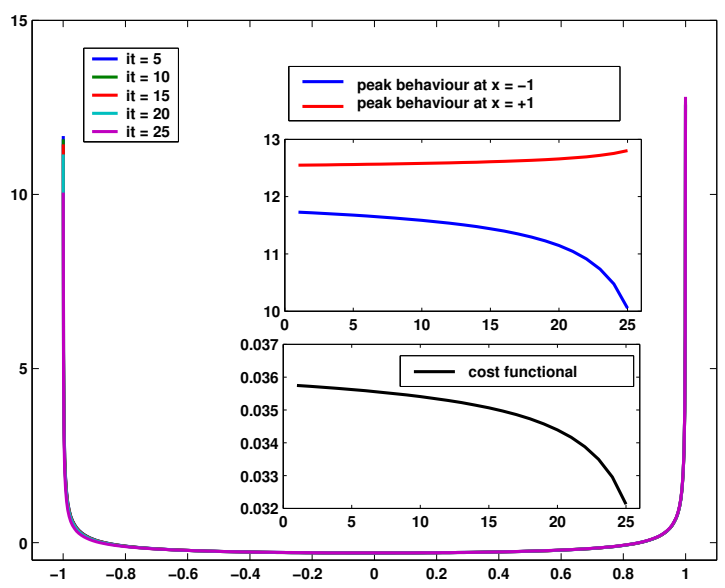

Figure 5.8: Evolution of the cost functional and the current distribution for the geometric evolution of Figure 5.7.

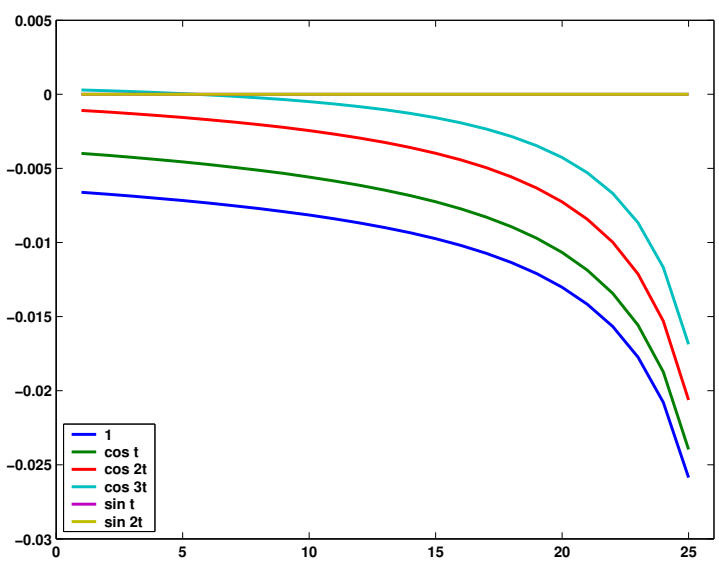

Figure 5.9: Evolution of the directional Fréchet derivatives for the geometric evolution of Figure 5.7.

is no evidence for this to happen from the evolution.

The general observations that have been discussed above re-appear for virtually every tested initial setup and for each tested positioning of the centre $x_{0}$ for $D$. Table 5.3 indicates that the initial setups given in Figure 5.10 become invalid after several iterations due to an overlap between $D_{x_{0}, r}$ and $\Gamma$, that the Euclidean norm of the directional Fréchet derivatives of the basis functions are increasing as the distance between the domain $D_{x_{0}, r}$ and the arc $\Gamma$ is decreasing, and that the homogenizing effect on the current distribution is much more pronounced on the near side of $\Gamma$ compared to the far side of $\Gamma$.

In a second example for the evolution we consider the optimization process with the choice of $\widetilde{T}_{n, k}$ as approximation space. Furthermore, we incorporate the Aug- 


\begin{tabular}{crrrrr}
\hline initial geometry & $\begin{array}{r}\text { breakdown } \\
\text { after steps }\end{array}$ & $\begin{array}{r}\text { reduction } \\
\text { of cost } \\
\text { functional }\end{array}$ & $\begin{array}{r}\text { reduction } \\
\text { of peak } \\
\text { at } x=-1\end{array}$ & $\begin{array}{r}\text { increase } \\
\text { of peak } \\
\text { at } x=1\end{array}$ & $\begin{array}{r}\text { increase } \\
\text { of norm of } \\
\text { gradient }\end{array}$ \\
\hline medium circle at $(-1.5,0)$ & 2 & $11.1 \%$ & $34.6 \%$ & $1.1 \%$ & $68.5 \%$ \\
small circle at $(-1.5,0)$ & 11 & $25.1 \%$ & $45.6 \%$ & $3.8 \%$ & $516.0 \%$ \\
large circle at $(-2.5,0)$ & 16 & $20.4 \%$ & $43.2 \%$ & $4.9 \%$ & $401.4 \%$ \\
large circle at $(-2.5,1)$ & 57 & $28.2 \%$ & $96.3 \%$ & $10.2 \%$ & $1377 \%$ \\
kidney at $(-1.5,0)$ & 35 & $25.6 \%$ & $79.4 \%$ & $6.7 \%$ & $228.4 \%$ \\
kidney at $(-1.5,-0.5)$ & 21 & $27.6 \%$ & $70.7 \%$ & $5.8 \%$ & $338.3 \%$ \\
peanut at $(-1.5,0)$ & 7 & $9.4 \%$ & $28.7 \%$ & $1.2 \%$ & $200.5 \%$ \\
peanut at $(-2,0.5)$ & 18 & $31.2 \%$ & $95.5 \%$ & $7.9 \%$ & $4588 \%$ \\
\hline
\end{tabular}

Table 5.3: Performance of the SDA without regularization or line search.
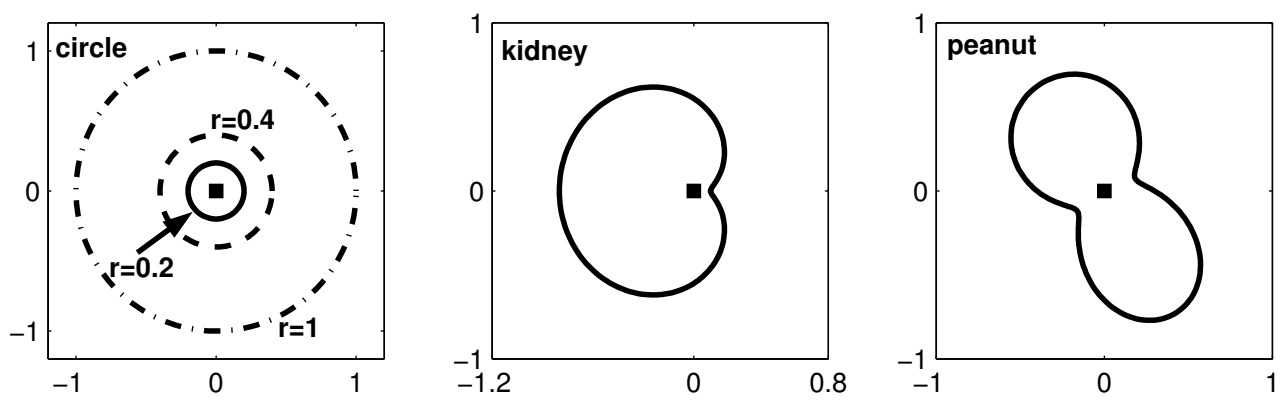

Figure 5.10: Tested initial geometric setups.

mented Lagrangian Approach into the functional. We emphasize that in the case of a symmetric configuration for $\widetilde{T}_{n, k}$ we need not set a hard constraint for the distance. Instead, we prescribe a lower bound on the distance. This approach works, as in the case of $\widetilde{T}_{n, k}$ the constant is the only basis function contributing to the distance for the direction $\vartheta=0$ (see Figure 4.1). Still, the choice of the minimum distance in the Augmented Lagrangian Approach cannot be arbitrary small. This is due to the way in which the system of integral equations (2.36) is solved. In solving (2.36) it is assumed that the off-diagonal operators have smooth kernels, which is true since $D_{x_{0}, r}$ and $\Gamma$ do not overlap. Nevertheless, the stability of the scheme depends on how good the composite trapezoidal rule approximates the kernels. In the case of very small distances between $D_{x_{0}, r}$ and $\Gamma$ the kernels display an almost singular behaviour and the approximation quality for the composite trapezoidal rule deteriorates and thereby creates instabilities. To some extent this behaviour can be encountered by finer discretizations of $\partial D_{x_{0}, r}$ and $\Gamma$, and by introducing substitution functions in parametrizing $\partial D_{x_{0}, r}$. We have found from our numerical experience that using 128 collocation points for the discretization of each of the contours $\partial D_{x_{0}, r}$ and $\Gamma$, the system can deal with a minimum distance of $d=10^{-3}$ without problems.

The geometric evolution for the second example is depicted in Figure 5.11. We see that the evolution draws the domain $D$ close to $\Gamma$. After 41 iterations the constraint becomes active, the minimal distance being set to $d=0.05$. Due to 


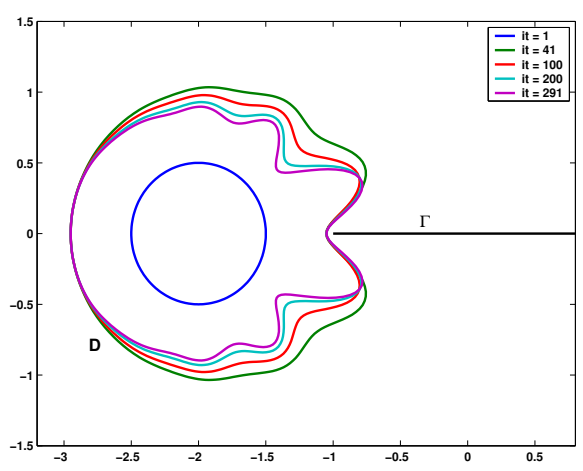

Figure 5.11: Geometric evolution for the SDA using $\widetilde{T}_{n, k}$ with $n=5, k=0$ and the Augmented Lagrangian Approach.
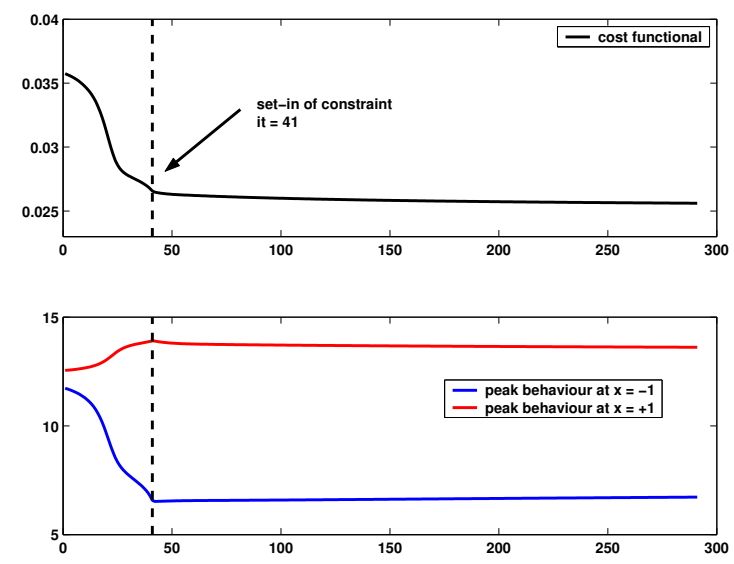

Figure 5.12: Evolution of the cost functional and the peaks of the current density for the geometric evolution of Figure 5.11.

the special form of the approximation space, $D$ already forms a cavity around $\Gamma$ which is slightly getting narrower in the remaining iterations. But this narrowing has only a very small effect on the cost functional as can be see from the upper graph in Figure 5.12.

Figure 5.11 shows furthermore that the narrowing of the cavity around $\Gamma$ induces oscillatory behaviour in the upper and lower parts of the boundary of $D$. This behaviour is undesired and also in a certain sense unnatural. This is revealed by an examination of Figure 5.13.

Here, we see that the directional Fréchet derivative with respect to the constant is the largest coefficient in the finite dimensional approximation to the gradient of the functional $F$. At the first iteration it clearly overrates all other components. This discreprancy becomes even more pronounced in the further evolution, showing that the formation of the cavity around $\Gamma$ is still of minor importance, and that the algorithm tries to make up for the constraint on the distance by narrowing the cavity. The evolution of the gradient in Figure 5.13 displays another 


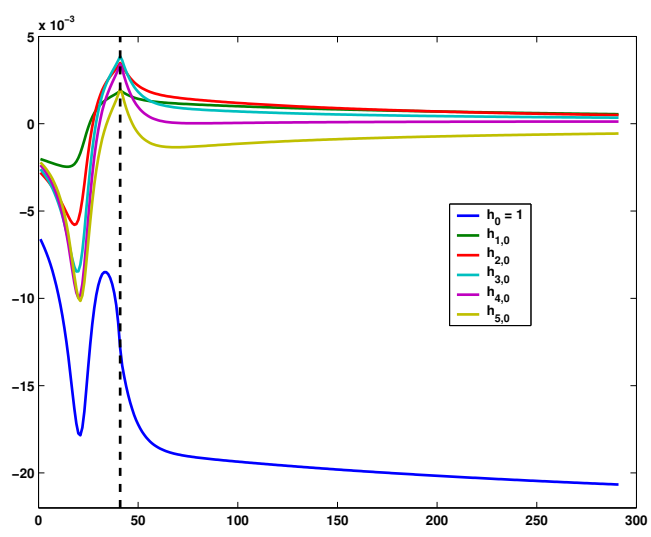

Figure 5.13: Evolution of the gradient for the geometric evolution of Figure 5.11.

feature which requires explanation. Contrary to the case of trigonometric polynomials the space $\widetilde{T}_{n, k}$ contains basis functions that are non-negative and oscillate symmetrically with respect to the angle $\vartheta=0$. Hence, the geometric evolution differs in its general appearance from Figure 5.7 in the sense that a cavity is building up right away. This formation becomes more and more pronounced as the boundary evolves until the tips of the cavity are located above or beneath the endpoint of $\Gamma$. From this time onwards the evolution of the cavity reduces rapidly, and the inner end of the cavity where only the constant basis function contributes can "catch up". This tendency eventually becomes so strong that the gradient changes the sign for the oscillatory basis functions. This induces a rapid increase in the directional Fréchet derivative of the constant basis function. Soon afterwards the constraint sets in preventing an overlap between $D$ and $\Gamma$. Now the Augmented Lagrangian Approach sets the directional derivative for the constant basis function to zero and the forming of the cavity around $\Gamma$ becomes the dominant feature in the evolution. The system then converges towards an equilibrium state in the further evolution.

Finally, in Figure 5.12 and Figure 5.14 we consider the current distributions for the iterates corresponding to the geometries depicted in Figure 5.11.

We observe especially from the upper graph in Figure 5.12 that the peak in the current density on the far side of $\Gamma$ undergoes only a mild increase, whereas the peak on the near side is significantly suppressed during the first steps of the iteration. As soon as the distance constraint has set in, the peaks stay almost constant for the rest of the iteration, although we observe that there is a slight tendency in the beginning to undo the evolution of the first iterations. In general, one can observe from Figure 5.14 that the overall shape of the current density is not changed in the evolution. Only a closer look at the right graph, which displays a zoom-in of the left graph, reveals that the narrowing of the cavity seems to underline the effect that has been visible also in the behaviour of the peaks. Namely, the redistribution of the current density towards the suppressed 

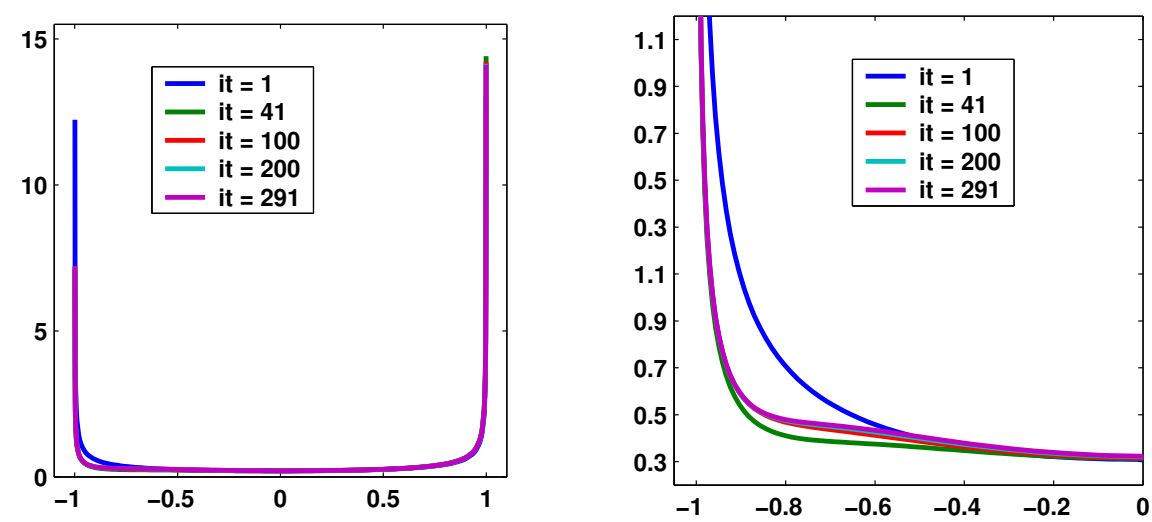

Figure 5.14: Evolution of the current density for the evolution from Figure 5.11.

peak. It seems that, as the distance on the side where the peak is suppressed is fixed, the influence of the increased peak at the far end does indeed influence the further evolution. The functional now seeks to reduce the peak on the far side as a further suppression of the peak at near side cannot be accomplished. Thereby it accepts a less homogeneous distribution in a region of $\Gamma$ that is comparatively far away from the left endpoint.

We close the discussion of the second numerical experiment again with a remark. We address the geometric evolution after the set-in of the constraint. In the above we have given reasons for the effect of the narrowing of the cavity. We point out that this narrowing does not only influence the general behaviour of the current density as it is depicted in the right graph of Figure 5.14, it also leads to oscillatory behaviour in the upper and lower part of the boundary of $D$. These effects have two consequences. Firstly, an effective homogenization of the current distribution can only be obtained by placing components of the domain $D$ at both endpoints of $\Gamma$ due to the local nature of the homogenization. Secondly, the suppression of regions with high curvature in the parametrization of $D$ is an issue which must not be neglected, as the optimal shape from Figure 5.11 is undesirable from an applicational point of view.

As a third example we include an examination illustrating the efficiency of both the curvature regularization and the Augmented Lagrangian Approach. The example has been computed using $T_{32}$ as approximation space, i.e. trigonometric polynomials of degree $\leq 32$. The initial setup is a circle of radius $r=0.999$, the center being placed at $(-2,0)$. The minimum distance between $D$ and $\Gamma$ has been fixed to $d=10^{-3}$ accordingly. Furthermore, we regularize oscillations in the boundary using the regularization term $G_{c}$ introduced in Section 4.2. The regularization parameter has been determined by trial and error, it is set to $\beta=10^{-6}$. The geometric evolution which is depicted in Figure 5.15 shows clearly the effects of the two regularization terms $G_{\vartheta, d}$ and $G_{c}$. The domain $D$ is kept at the prescribed distance $d$ from the arc $\Gamma$. As before, a cavity is building out im- 


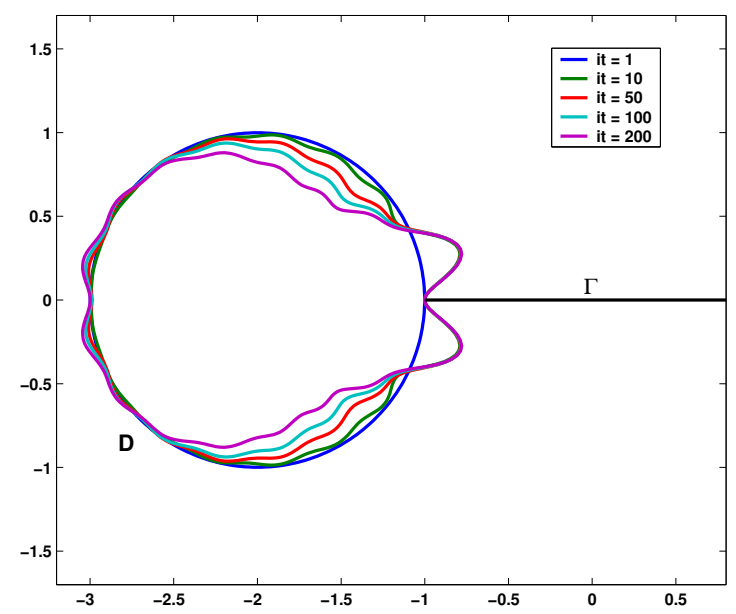

Figure 5.15: Geometric evolution for the SDA using curvature regularization with regularization parameter $\beta=10^{-6}$ and the Augmented Lagrangian Approach with minimum distance $d=10^{-3}$.

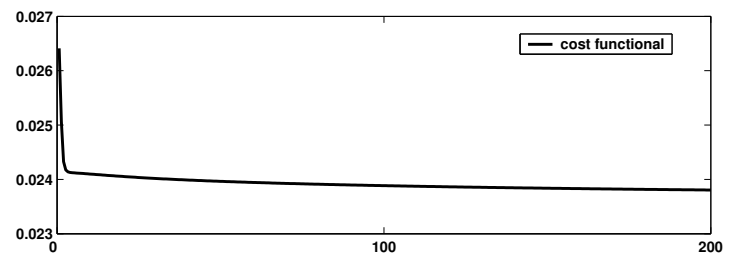

Figure 5.16: Evolution of the cost functional for the geometric evolution from Figure 5.15.

mediately. It has reached its full extent already after ten iterations. From then on, the regularization of the curvature governs the evolution flattening the shape of $D$ at the upper and lower boundary. In the region of the boundary facing $\Gamma$ the influence of the functional $F$ is nevertheless stronger than the effect of the regularization. Hence, here regions with large values for the curvature remain present. The evolution of the objective functional for the corresponding situation is displayed in Figure 5.16.

We observe a pronounced reduction in the current distribution in the first iterates when the cavity is forming. Afterwards the reduction slows down considerably, but continues up to the $200^{\text {th }}$ iterate, where the evolution becomes stable. At the same time the gradient of $F$ remains still large since the original functional still displays the tendency to draw $D$ nearer to $\Gamma$ which is prevented by the Augmented Lagrangian Approach.

After these illustrations for the necessity for the regularization terms as well as for their feasibility, we now turn to the comparison of the computed optimal shapes for different sets of parameters. 

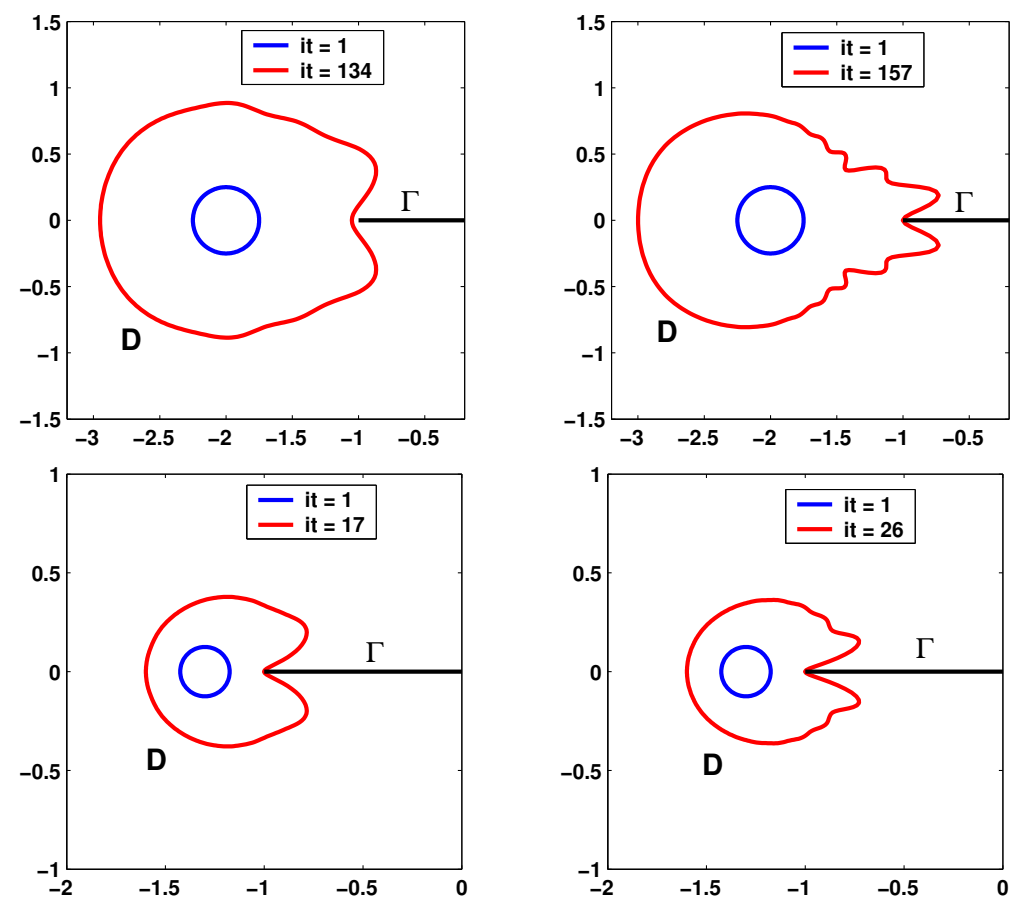

Figure 5.17: Optimal shapes for different sets of parameters using $\widetilde{T}_{n, k}$ as approximation space.

Let us explain and discuss the results of Figure 5.17 and Figure 5.18 in some detail. In the examples of Figure 5.17 we have used the approximation space $\widetilde{T}_{n, 0}$ for two different values of the dimension $n$ and for different values of the regularization parameter $\beta$ for the curvature regularization.

In the graph on the left-hand side of the top row we have $n=8$, and additionally we have employed curvature regularization with $\beta=10^{-6}$. On the right-hand side we have $n=32$ and $\beta=0$. We see that the higher dimension of the approximation space leads to a narrower cavity of $D$ around $\Gamma$. At the same time the larger approximation space contains also more oscillatory basis functions which is clearly visible in the plot on the right-hand side.

In the bottom row we have started with a smaller initial setup. Here, we have used $n=8$ basis functions, and we consider curvature regularization with the regularization parameter being set to $\beta=10^{-6}$ on the left-hand side and $\beta=0$ on the right-hand side. The comparison here reveals that the curvature regularization acts as a counterweight against making the cavity narrow, but this is expected as the cavity is a region with high curvature. We note again, that the distant constraint for the Augmented Lagrangian Approach is again only a lower bound on the distance, it is set to $d=0.001$. This implies that the most reduction in the cost functional can be obtained by drawing $D$ nearer to $\Gamma$. We can observe furthermore that the general appearance is the same for all experiments. 
The current distributions look always similar to the one displayed in Figure 5.14. Correspondingly, the objective functional stabilizes at values around $\approx 0.024$. Expectedly, the values are slightly larger $(\approx 0.026)$ in the case of curvature regularization.

In the examples of Figure 5.18 we have used the approximation space $T_{n}$ (i.e. trigonometric polynomials), again for different values of $n$ and for different values of the regularization parameter $\beta$ for the curvature regularization.
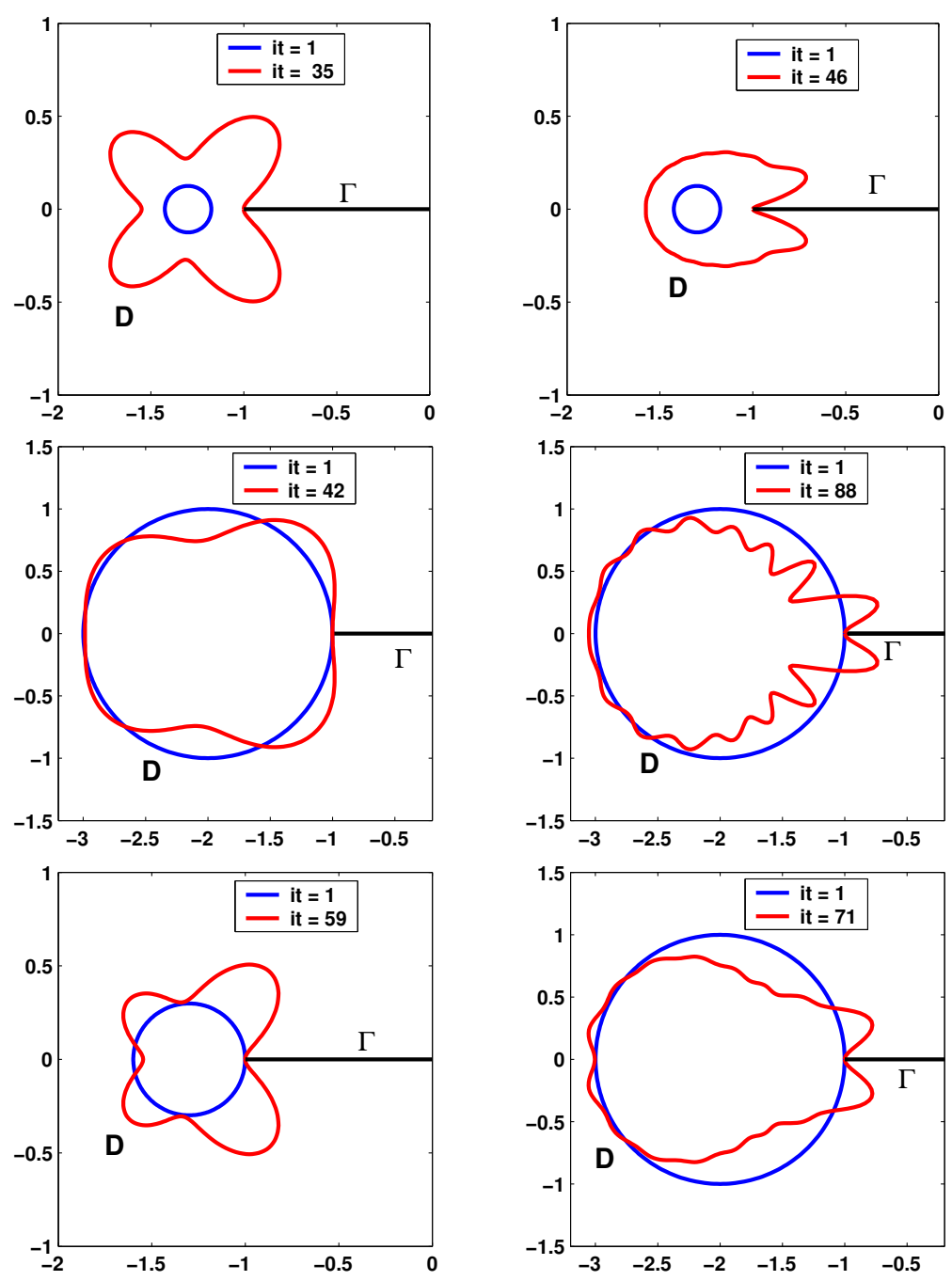

Figure 5.18: Optimal shapes for different sets of parameters using $T_{n}$ as approximation space.

The top row compares the results for the approximation space $T_{n}$ for values of the dimension being $n=8$ on the left-hand side and $n=32$ on the right-hand side. In the case of trigonometric polynomials we realize the Augmented Lagrangian Approach via a hard constraint on the minimal distance. It is again set 
to $d=0.001$. Note that we have used an initial setup in the top row that does not satisfy this hard constraint. The adaptive choice of the regularization parameter $\mu_{n}$ from (4.30) then yields a geometry which satisfies the constraint already in the second iterate. Additionally, we have applied curvature regularization with $\beta=10^{-6}$ on the right-hand side. We notice that in the case of trigonometric polynomials a small approximation space with essentially non-oscillating basis functions does not work satisfactorily. Opposed to that, oscillations can be damped out efficiently using the curvature regularization. At the same time the rapidly oscillating basis functions enable the forming of a rather narrow cavity around the arc $\Gamma$.

In the center row we have repeated the experiment for a different initial setup, namely a circle with a larger radius of $r=0.999$ around $(-2,0)$. The lefthand side shows that the basis functions of the approximation space just do not oscillate fast enough to form a cavity around $\Gamma$. The right-hand side shows that even for $n=32$ and $\beta=0$ we cannot obtain the same narrowness of the cavity as above. This is mainly due to the fact that all coefficients in the finite-dimensional representation of the radial function are large, because the distance between $\Gamma$ and the centre of $D$ is larger.

Finally, in the bottom row we compare the efficiency of the curvature regularization for different setups. The setup on left-hand side coincides with the setup of the left-hand side of the top row, the setup on the right-hand side coincides with the setup of the right-hand side of the second row. In both cases we have additionally employed curvature regularization, the regularization parameter being set to $\beta=10^{-6}$. We observe that the curvature regularization has nearly no effect on the left-hand side, whereas the effect is rather drastic on the right-hand side. The reason for these differences is that in the larger approximation space that has been used for the example on the right-hand side, there are enough degrees of freedom left to suppress the oscillations in the boundary effectively without destroying the cavity around $\Gamma$. In the experiment on the left-hand side all degrees of freedom have to be employed to build up the cavity such that the curvature regularization remains without effect.

Comparing the results for the different approximation spaces we see that for domains with one connected component small initial setups in combination with a large dimension for the approximation space give the best results from a visual point of view. From our perspective this is the critical criterion as the optimal shapes are all comparable with respect to the value of the objective functional, and they also yield qualitatively similar current distributions which basically resemble Figure 5.14. Furthermore, the value $\beta=10^{-6}$ has proved to be a fairly good choice which balances the forming of a cavity and the suppression of oscillations in the boundary. Finally, we observe that the curvature regularization generally seeks to flatten the upper and lower part of the boundary thereby indicating how a simpler geometry may look like that can be manufactured for 


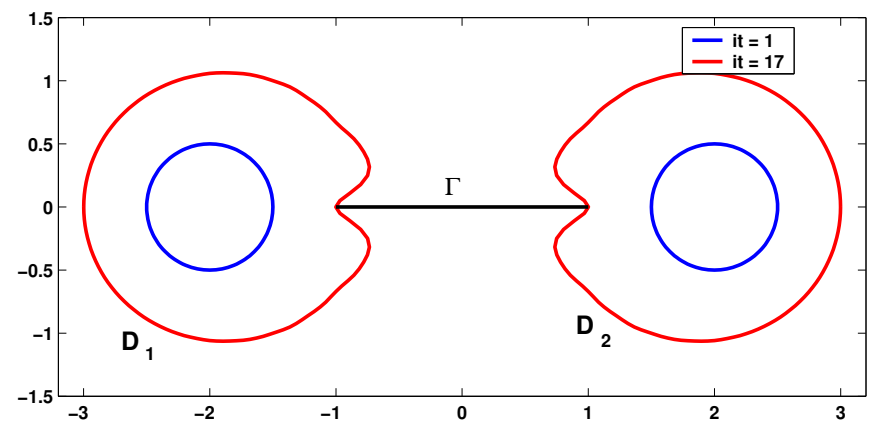

Figure 5.19: Optimal shape for a domain consisting of two connected components.

physical experiments.

With these optimal shapes we finish the examination into the nature of the approximation spaces and the curvature regularization. For the remainder of this section we consider a setup consisting of two symmetrically placed components for $D$. We judge this setup as the most relevant for the application by the reasoning in the beginning of this section.

In Figure 5.19 we see the initial and the optimized shape for a domain $D$ consisting of two connected components $D_{1}$ and $D_{2}$. For each component we have chosen the space $\widetilde{T}_{12,1}$ of the especially designed basis functions from Section 4.2. Furthermore, we have employed curvature regularization with a regularization parameter $\beta=10^{-6}$, the minimal distance has been set to $d=0.001$.

We see that as in the case of only one connected component, the components are drawn towards the arc by the evolution. As soon as the distance constraint from the Augmented Lagrangian Approach sets in, a cavity is evolving, which is balanced by the curvature regularization. The main feature here is, that the cavity is much less pronounced than in the case of only one connected component. The reason for this is given by an examination of evolution of the functional in Figure 5.20 and the corresponding current densities in Figure 5.21.

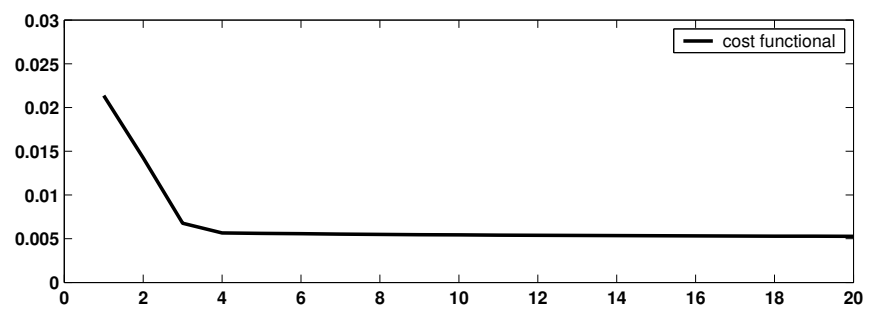

Figure 5.20: Evolution of cost functional for geometric evolution of Figure 5.19.

We see that the value of the cost functional is not only smaller compared to Figure 5.16, where the optimization has been carried out using a domain with one connected component, but also the reduction is much more pronounced. 

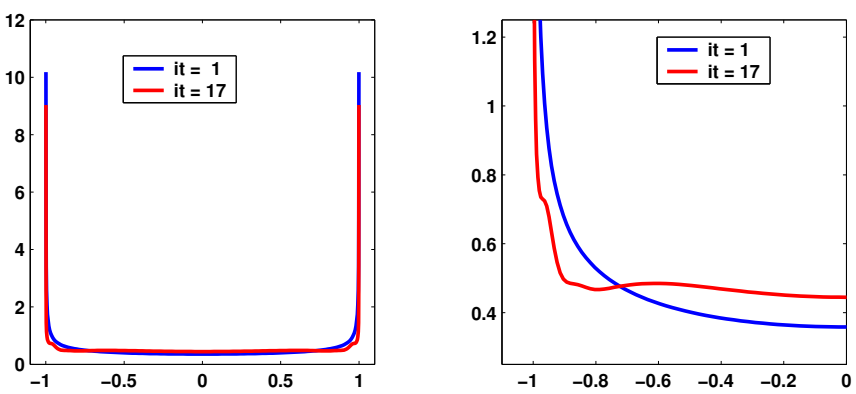

Figure 5.21: Current distributions for initial and optimized shape of Figure 5.19.

In the experiments with one connected component for $D$ the value of the cost functional could be reduced by a factor $>0.5$, here the factor is $\approx 0.25$.

Similarly, in Figure 5.21 we see that the two components induce a considerable reduction of the peaks in the current distribution at both endpoints of $\Gamma$. In view of what we have said above, the cavity consequently does not need to try to reduce the peak at the other end. Instead, it is employed just to flatten the current distribution in the regions near the endpoints. The reduction of the peaks is not as pronounced as before, since now there is a re-distribution from both side towards the other which in a way cancels out. Still we see from the zoomed in graph on the right-hand side of Figure 5.21 that a considerable redistribution towards the centre of $\Gamma$ has taken place. This is a particular difference to the case of only one connected component, where no effect has been visible at $x=0$ (see Figure 5.14). Furthermore, the local values of the current distribution at the first and at the final iterate display the same behaviour in a region around the centre. The major differences happen in the interval $[-1,-0.5]$ and similarly in $[0.5,1]$ due to the symmetry, where the current distribution for the optimized geometry has a local maximum near $x=-0.6$. From there the local values are again decreasing until about $x=-0.9$, where the formation of the peak sets in. This change in the overall behaviour of the current distribution is the most important feature of the optimized system, and it is in accordance with the overall aim of the scheme. The current distribution is less inhomogeneous over the entire arc, and the local current values near the endpoints are reduced. This is not only meant with respect to the peak which is forming nevertheless, but also for the local current values that are in a still local although larger region around the endpoint. We observe that the reduction amounts up to a factor of ten compared to the current density for an arc without any domains being placed in its vicinity. This has also been approved in [43].

We conclude this section with a short comparison of additional, physically relevant quantities, namely the distribution of the horizontal and vertical components of the magnetic field. The distribution of the horizontal component is given in Figure 5.22, the vertical component is displayed in Figure 5.23. In both figures 

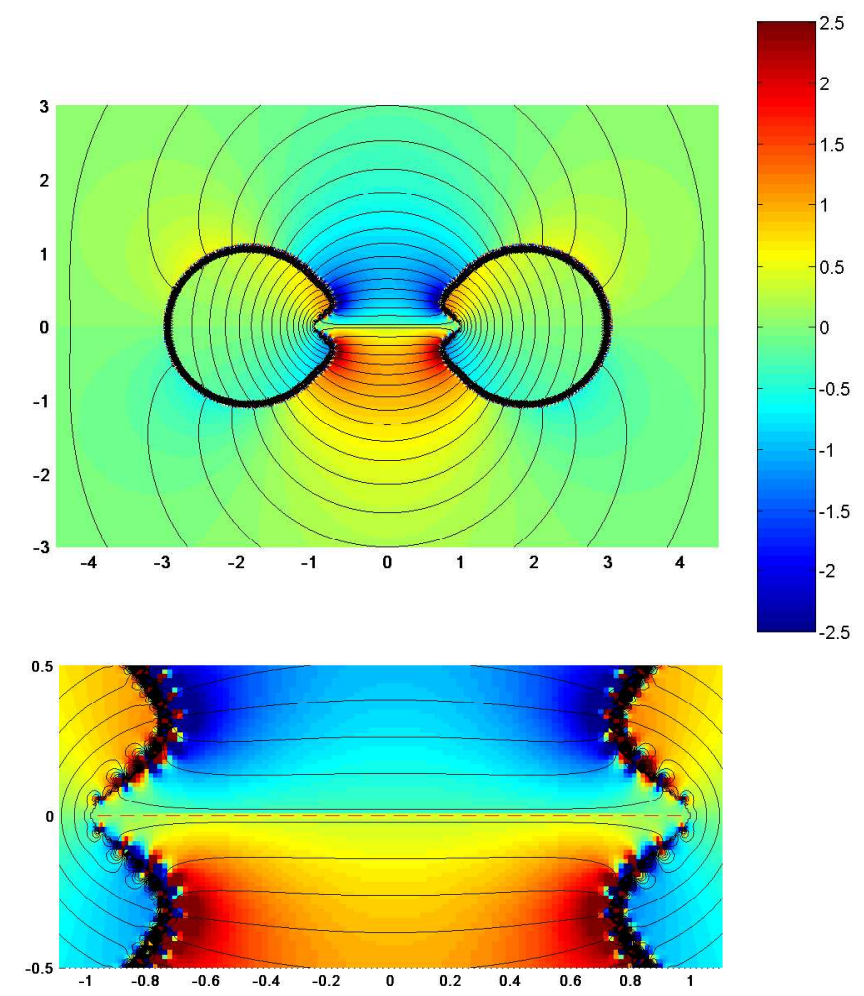

Figure 5.22: Distribution of the horizontal component of the magnetic field for the optimized geometry of Figure 5.19. The lower graph is a zoom-in of the upper, where the red dashed line denotes the position of $\Gamma$.

the isolines of the potential are visible in the form of the magnetic field lines.

In Figure 5.22, the current density which corresponds to the jump in the horizontal component of the magnetic field across $\Gamma$ is clearly visible and more pronounced than in Figure 5.3. This clearly indicates the redistribution of the local current towards the centre of the arc and can be seen best from the lower graph which is a zoom-in of the upper one. Nevertheless we observe that the magnetic field becomes strong at the tips of the cavity while it is small at the inner end.

Figure 5.23 accordingly displays the vertical component of the magnetic field, the lower graph being again a zoom-in of the upper. Before discussing the features in more detail, we remark again that the values for the magnetic field in the components of $D$ are not displayed correctly. We have suppressed the necessary multiplication with the magnetic permeability for the sake of a better visualization. We see that the vertical component of the magnetic field is particularly strong in the cavity, which seems to be similar to the case where no domain is present in the vicinity of $\Gamma$ (see left-hand side of Figure 5.4). The field lines reveal that the reason here is completely different. As mentioned in Section 5.1 the domain sucks in the magnetic field lines. This has the consequence that in the 

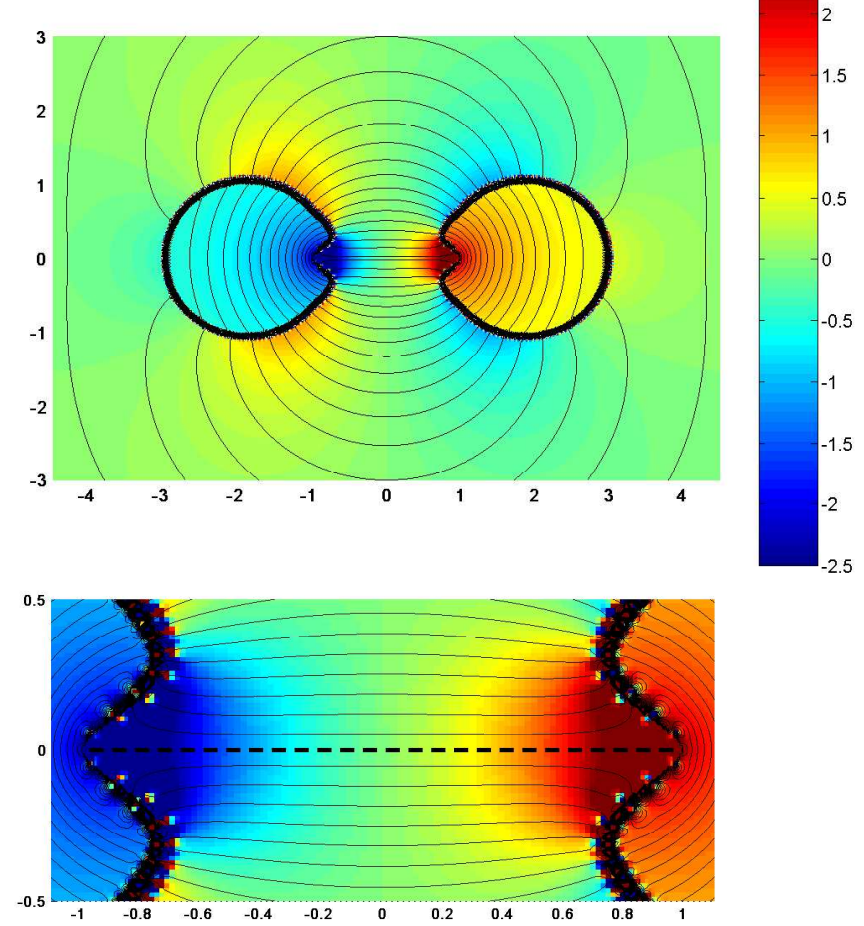

Figure 5.23: Distribution of the horizontal component of the magnetic field for the optimized geometry of Figure 5.19. The lower graph is a zoom-in of the upper, where the black dashed line denotes the position of $\Gamma$.

present example no ellipse-shaped field lines appear around the endpoints of $\Gamma$ on a visible scale, but all of them end in the boundary of $D$. This corresponds to a drastic suppression of the magnetic field directly at the endpoint, and thus enables much stronger currents to be transported by $\Gamma$ in this setup.

We can conclude that the Steepest Descent Approach satisfies the requirements of the physical application and the mathematical model very well, and that it has given clear ideas on how geometries for the magnetic environment should look like that allow an enhancement of the current carrying capability. Nevertheless, further tuning and numerical experiments are necessary to incorporate features into the geometric design that make the optimal shapes appropriate for manufacturing such that these theoretical results can be verified in experimental physics.

In preview of the examples from the following section we would like to add two arguments for not considering non-symmetrically placed domains with respect to the line defined by $\Gamma$. First of all, the specialized shape of the approximation space $\widetilde{T}_{n, k}$ can only be used sensibly in a symmetric setup due to the strongly oscillatory behaviour of the basis functions. Otherwise, this would render the 
Augmented Lagrangian Approach effectless. And secondly, when employing the space $T_{n}$ of trigonometric polynomials, we have to impose a hard constraint, i.e. fix the distance between $\Gamma$ and $D$ as shown in Figure 4.2. This already causes the results obtained in that way to be not comparable with the results which we are going to obtain via the Level Set Approach in the following section.

\subsection{The Geometric Optimization Problem via the Level Set Approach}

In this section we present evidence for the ability of the Level Set Method presented in Section 4.3 to succeed in numerically solving the Geometric Optimization Problem 3.3.

Therefore, we give examples for the feasibility of the contouring and the extension algorithm. In the course of these studies we will see a deficiency in the scheme, namely the unsmooth spacing of boundary points, that might cause larger numerical errors in the boundary integral method, and consequently render optimized shapes of inferior quality in comparison with the Steepest Descent Approach.

The section is closed with an example for the feasibility of the algorithm. Here, we restrict ourselves to the presentation of the physically relevant situation of a domain $D$ with two connected components, that are placed symmetrically at the endpoints of $\Gamma$.

But let us consider the central steps of the Level Set Algorithm 4.18 first. In Section 4.3 we have argued that it needs two additional steps in order to relate boundary integrals and level sets. One of these necessary steps was the resolution of the 0-level set, a task which is normally avoided in Level Set Methods.
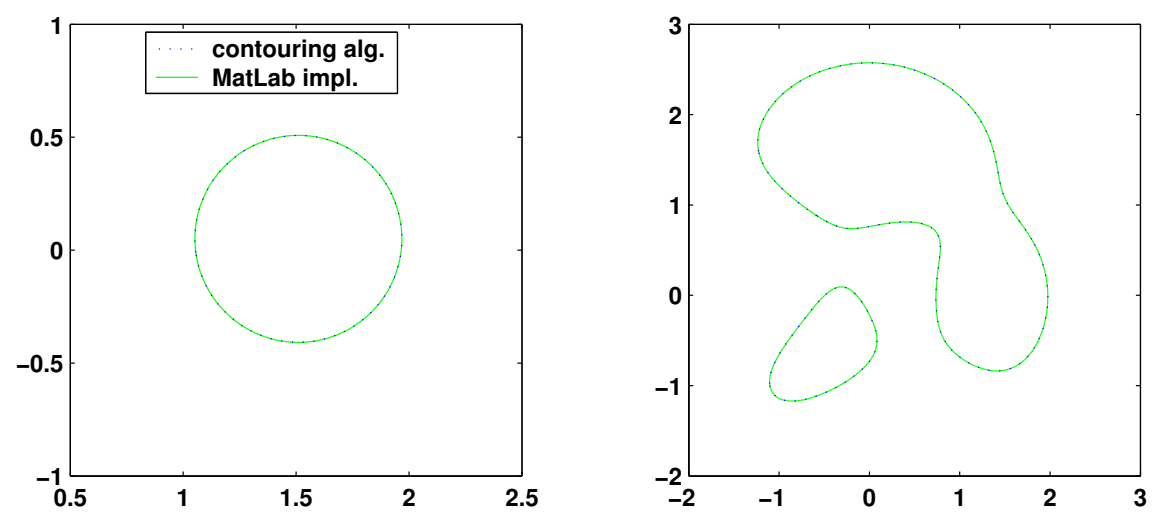

Figure 5.24: Comparison of the contouring algorithm with the standard MatLabimplementation for two examples. 
In Figure 5.24 we compare the performance of the contouring algorithm that we have proposed in Section 4.3 with the standard MatLab-contouring algorithm. Both algorithms accept the discretized version $\phi^{n}$ of the level set function as input and produce an ordered set of points as output that is an approximation to the 0-level set. The difference between both algorithms is that the MatLabimplementation obtains the points on the boundary by linear interpolation along the grid lines, whereas our contouring algorithm returns boundary points that are in the interior of the grid cells. This makes a numerical comparison impossible as the generated sets of boundary points are not only different, they are even meant to have no points in common. The only way of comparing the two generated contours is a visual comparison.

The result is that both for the simple circular geometry on the left-hand side of Figure 5.24 and also for the more complex geometry on the right-hand side the contours match perfectly. The geometry in the graph on the right-hand side has been generated using the peaks-function from MatLab. This matching between the two algorithms is again illustrated in Figure 5.25, where we have zoomed in to interesting regions of the boundary contours for the examples of Figure 5.24.
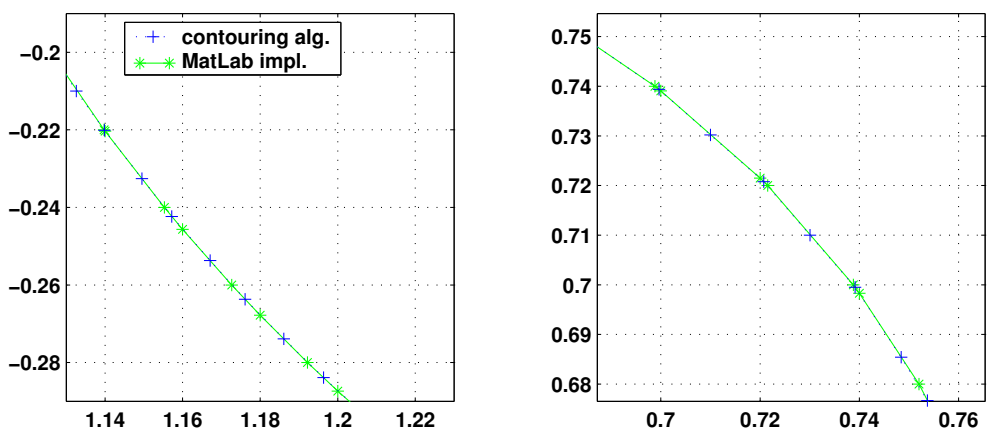

Figure 5.25: Comparison of the contouring algorithm with the standard MatLabimplementation for two examples (zoom-in of Figure 5.24).

Figure 5.25 also reveals two important features of the two algorithms. Let us first focus on the MatLab-implementation. From the graph on the right-hand side we see that interpolation along the grid lines as it is done in the MatLabimplementation may lead to an extremely irregular spacing of the boundary points. Directly at the centre of the graph on the right-hand side we see that the 0 -level set is crossing two grid lines very near to the grid point $(0.72,0.72)$. These intersections are marked with the green stars. At the same time the contouring algorithm places just one boundary point in that region which is marked by the blue '+'-sign. This feature of extremely irregular spacing of grid points has been one of the central reasons why have proposed this other kind of method.

Nevertheless, the contouring algorithm is also not too robust against irregular spacing of boundary points. This is illustrated in Figure 5.26. 

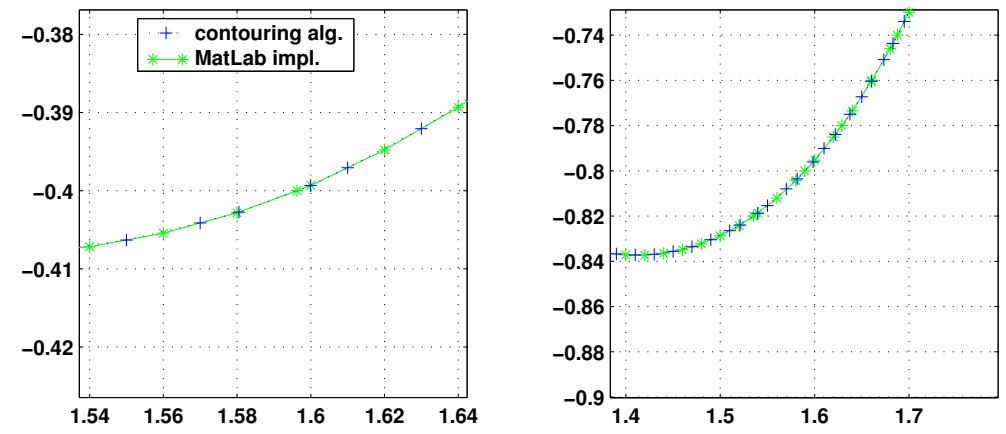

Figure 5.26: Comparison of the contouring algorithm with the standard MatLabimplementation for two examples (zoom-in of Figure 5.24).

In both graphs we see that kinds of pairs of boundary points are forming. The effect is less pronounced than in the standard MatLab-implementation, but it is still visible. The irregular spacing becomes even more clear if we display the distance between neighbouring grid points as an approximation to the line element. Using Taylor expansion for the parametrization $x$, we have the approximation

$$
\left\|x\left(t_{k+1}\right)-x\left(t_{k-1}\right)\right\| \approx\left(t_{k+1}-t_{k-1}\right)\left\|x^{\prime}\left(t_{k}\right)\right\|
$$

which we employ as an approximation for $\left\|x_{k}^{\prime}\right\|$. Now if the points $x_{k}$ come from a smooth parametrization with regularly spaced $t_{k}$, we expect that also the lefthand side of the above displays the same smoothness. For example, in the case of the standard parametrization of the circle with equidistantly spaced $t_{k}$, both the right-hand side and the left hand side are constant. For the contouring algorithm this unfortunately is not the case as Figure 5.27 shows.

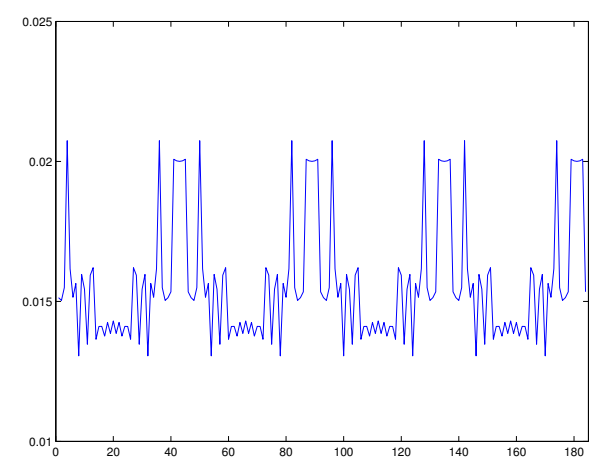

Figure 5.27: Distances $\left\|x_{k+1}-x_{k-1}\right\|$ for the boundary points $x_{k}$ that are obtained by the contouring algorithm for the circle.

This reveals the severest drawback of the Level Set Algorithm in the form we present it. The unsmooth approximation to the line element first of all leads to deterioration of the boundary integral method. Secondly, the evaluation of 

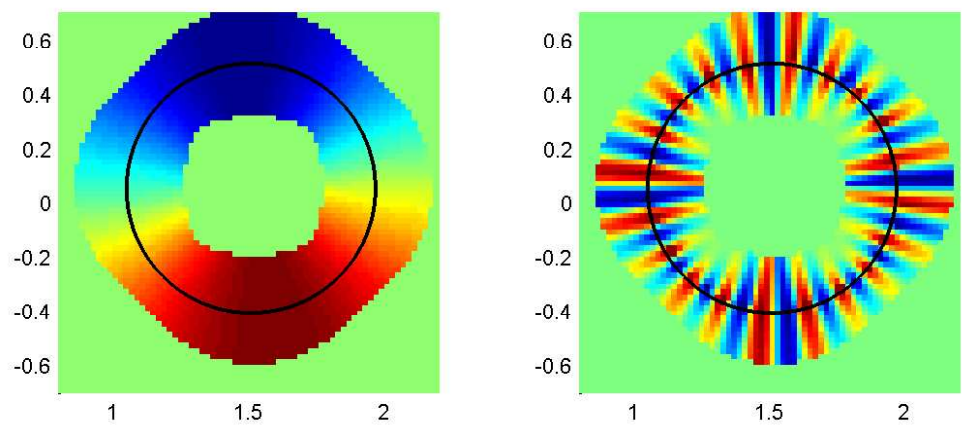

Figure 5.28: Examples for the feasibility of the extension algorithm for artificial 'boundary' velocities. The velocities are sine functions of periodicity $2 \pi$ (left) and periodicity $0.1 \pi$ (right). The position of the 0-level set is marked by the black line

the Fréchet derivative requires the evaluation of the second derivative for the unknown perturbation (see Lemma 3.29). As the perturbation is unknown we have to approximate the second derivative using finite differences. Here now, the unsmooth approximation to the line element enters a second time, but now as a quadratic term, which induces even more numerical error.

The evaluation of the Fréchet derivative leads to the second algorithm we have proposed as an additional step for the Level Set Algorithm, namely the extension algorithm. In the following, we give two illustrations for artificial 'boundary' velocities for the case of a circular domain.

We see in Figure 5.28 how the extended velocities look like. The one on the left-hand side corresponds to a 'boundary' velocity given by a sine function with periodicity $2 \pi$, the one on the right-hand side to a sine function with periodicity $0.1 \pi$. The figures display clearly that the extended field is constant normal to the 0-level set which is marked by the black line. In the faster oscillating 'boundary' velocity on the right-hand side we can also observe a slight displacement between the inner and the outer side. This is partly due to the colourcoding of MatLab, but also to the fact that the values of the extended velocity at two gridpoints that are connected via a grid line crossing the 0-level set are influenced by different boundary points when the 0-level set is roughly diagonal to the grid. This can be seen also from Figure 4.11 a). Here, the left grid point on the centre grid line is influenced only by $\bar{x}^{(1)}$, whereas the central grid point is influenced by $\bar{x}^{(2)}$ and $\bar{x}^{(3)}$. In the case of rapidly oscillating 'boundary' velocities the values might already differ considerably, which explains the displacement.

As a second example for the feasibility we display in Figure 5.29 the extended velocity that corresponds to the Fréchet derivative of $F$ from (3.4). The position of the domain is marked by the black line, the $\operatorname{arc} \Gamma$ is placed to the left of $D$. As 


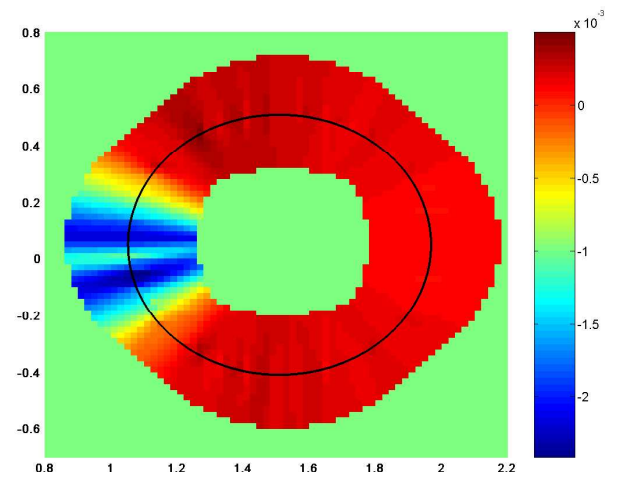

Figure 5.29: Example for the feasibility of the extension algorithm. The 'boundary' velocity is given by Fréchet derivative of the objective functional $F$ from (3.4), the position of the 0-level set is marked again by the black line

before, it is a line segment of length 2 lying parallel to the first coordinate axis, the right-hand side endpoint being located at $(1,0)$.

We observe that the general behaviour of the Fréchet derivative agrees with the behaviour we have encountered in the previous section. The derivative is very small on the side not facing the arc $\Gamma$ and it develops strong peaks that are rather localized in the regions that are directly facing $\Gamma$. Moreover, we observe that the derivative is strongest not directly in the direction of $\Gamma$, where it has a weak local minimum. We conclude that the Fréchet derivative seeks to evolve the domain towards a flat surface that is perpendicular to $\Gamma$.

Before considering and presenting the optimal shapes for the Geometric Optimization Problem that are obtained using the Level Set Approach, we have to address another issue, which has already played a central role in the Steepest Descent Approach. As the Steepest Descent Approach, the Level Set Approach does not automatically incorporate the separation between $D$ and $\Gamma$, but it is much easier to include this feature. The idea is to mask the extended velocity which induces the evolution of the 0-level set. We simply set the velocity to zero in a small region around $\Gamma$. This has the effect that the level lines of the level set function $\phi$ stay constant with respect to time in that region. As the 0-level set initially is not in this region it cannot enter it during the evolution. Thus, it is kept away from the arc $\Gamma$. The process of masking is illustrated in Figure 5.30 for the case of the artificial 'boundary' velocity given by the cosine function.

This masking process is very easy and efficient as long as the masked area remans simple. For the masking of more complicated geometries one can also use level set functions in combination with the Heavyside function as a mask. This leads to the masking of a domain which is defined via the usual definition of a domain in the context of Level Sets.

After these preparatory steps we now present the results for the Geometric Opti- 

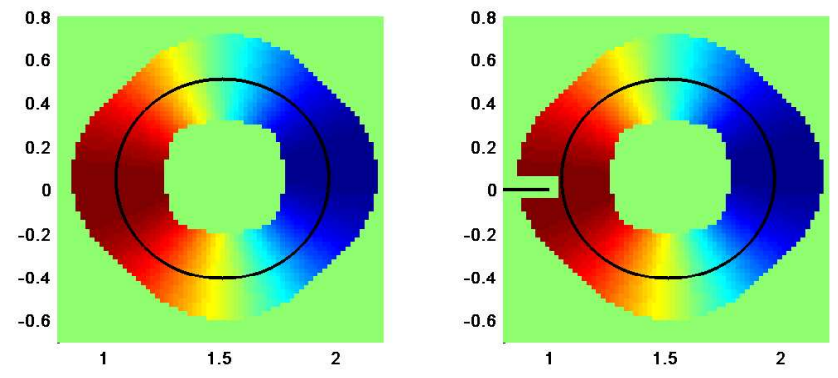

Figure 5.30: Example for the masking of the extended normal velocity. The artificial 'boundary' velocity is given by a ccosine function, the positions of $\Gamma$ and the 0-level set are marked again by the black lines. The left-hand side graph shows the unmasked velocity, the graph on the right-hand side shows the masked velocity.

mization Problem using the Level Set Approach. The example we present is the analogue to the symmetric example from Section 5.2. The domain $D$ is given by two ellipse-shaped components that are placed symmetrically at both endpoints of the arc $\Gamma$, which is again a line segment parallel to the $x_{1}$-axis with the enpoints being at $( \pm 1,0)$. The mesh width of the the level set grid is $h=0.05$, the masked area is a rectangle of width $W=2.042$ and height $H=0.102$ being placed symmetrically with respect to the origin. The step size for the level set evolution is chosen adaptively according to the Courant-Friedrichs-Levy condition (4.61). The physical parameters are chosen as usual. We have set $I=1$, and $\mu_{e}=1, \mu_{1,2}=50$. The arc $\Gamma$ is discretized using 129 collocation points. The geometric evolution of the system is depicted in Figure 5.31, the corresponding evolution of the cost functional is displayed in Figure 5.32.

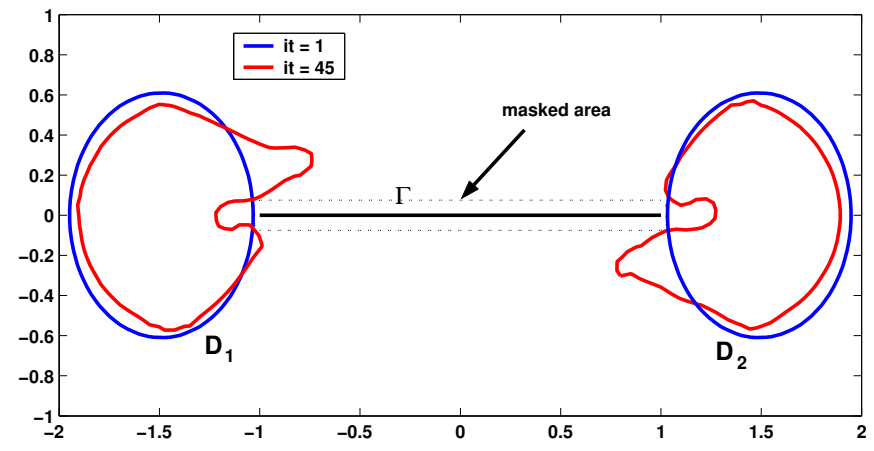

Figure 5.31: Optimal shape for the Geometric Optimization Problem using the Level Set Approach.

Let us first examine the evolution of the cost functional from Figure 5.32. We see that the decrease in the cost functional is not as steady as in the Steepest Descent 


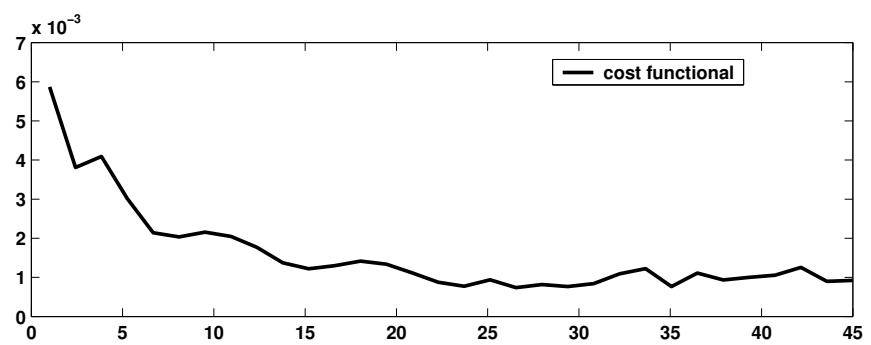

Figure 5.32: Evolution of the cost functional for the geometric evolution via the Level Set Approach given in Figure 5.31.

Approach. Nevertheless, the values of the cost functional are comparable to the values for the Steepest Descent Approach given in Figure 5.20. As the functional is not monotonically decreasing, we have to be careful when to stop the iteration. In the example above we have stopped as soon as the differences in the means of the values of the cost functional fell below a certain threshold.

Turning to Figure 5.31, we see that contrary to the Steepest Descent Approach a non-symmetric solution for each component of $D$ evolves. Still, viewing the two components together we also observe that one is the rotation by $180^{\circ}$ of the other. The reason for this is that in an early period non-symmetrical features appear in $D_{2}$, that we judge to be the effect of numerical errors in the Fréchet derivative due to the irregular spacing of the boundary points addressed above. This is displayed in Figure 5.33.

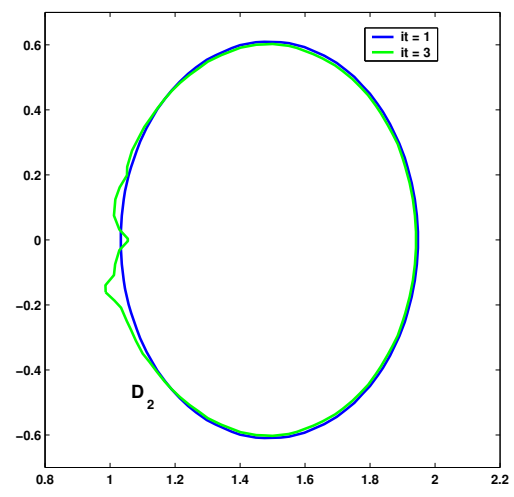

Figure 5.33: Non-symmetric geometric evolution in the Level Set Approach due to nummerical errors.

Here we see, how on the left-hand side of $D_{2}$ the symmetry is locally disturbed. The disturbances are limited to one or two boundary points which supports our impression. In the course of the iteration the system is not able to recover this error. Consequently also the other component builds out the same non-symmetric feature to restore the symmetry of the whole system. This phenomenon indicates that symmetric configurations as considered in the previous section are unstable, 
although we have experienced no such instabilities to occur in the framework of the Steepest Descent Approach. The reasons for this are not fully clear. We think that the numerical errors are weighted stronger in the Level Set Approach as the basis functions only have local support.

Turning back to the optimal shape in Figure 5.31 we also notice a second difference to the optimal shape from the Steepest Descent Approach in Figure 5.19. In particular, we observe that the boundaries of $D$ have not evolved towards the endpoint of $\Gamma$, but away from it. Here again, we can only speculate about the reason. First, we note that the optimal shape in Figure 5.19 has been computed only with a lower bound on the distance. Hence, the system there also had the possibility of separating the $D$ and $\Gamma$ further. As this has not happened, the only reason we can adduce is again the global support of the basis functions, in the sense that the tendency to form a cavity around $\Gamma$ is so strong that it dominates the tendency of separating $D$ and $\Gamma$ at the endpoint. Now the local support of the basis functions in the Level Set Approach is able to distinguish between these two tendencies. Furthermore, the conformal mapping example from Figure 4.3 supports this idea, as it also shows that in forming the cavity, the boundary $D$ is drawn away from $\Gamma$ at the endpoints.

Finally, we consider the current distributions for the initial and the optimal shape. They are displayed in Figure 5.34.
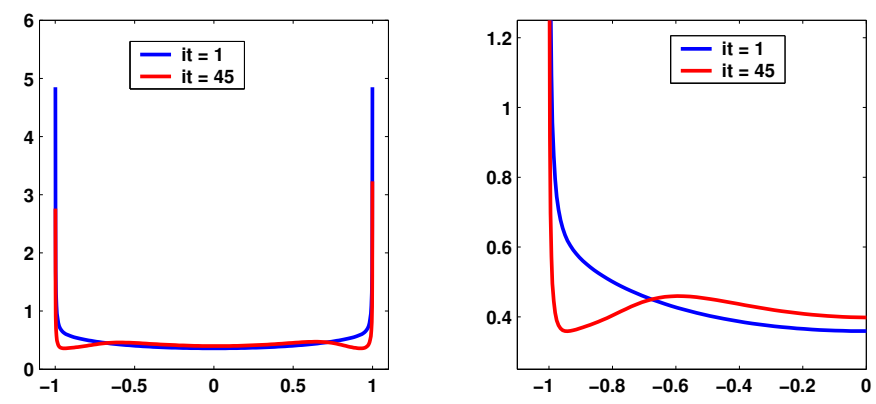

Figure 5.34: Current distribtions for the initial and optimal shape of Figure 5.31.

Here, we see that the behaviour of the current density is similar to the ones obtained by the Steepest Descent Approach which are given in Figure 5.21. We see in the zoom-in on the right-hand side, that the general behaviour of the initial distribution and the optimal distribution coincide in the centre. Furthermore, the formation of the peak is unaffected although a suppression of the peak is clearly visible. Again, the interesting feature is the local maximum of the current distribution near $x=-0.6$ and the strong decrease until $x \approx-0.95$. These features show again that the Geometric Optimization Problem succeeds in homogenizing the current distribution and thus allows a transports of transport currents that are several times larger than for an unshielded superconducting film. 


\section{Chapter 6}

\section{Discussion and Outlook}

This final chapter is devoted to a comparison of the numerical approximation schemes that we have presented in Section 4.2 and Section 4.3, and for which we have given numerical examples in the corresponding sections of Chapter 5 . We will also indicate how the ideas of this thesis can be built upon and extended.

\subsection{Discussion}

The previous chapter has given ample opportunity to compare the performance of the two approaches for a numerical approximation to the Geometric Optimization Problem that have been presented in Section 4.2 and Section 4.3. It is the the purpose of this finishing section to contrast both approaches reviewing their advantages and drawbacks.

We start our considerations by a closer look at the Level Set Algorithm. Already from the introduction the clear and easy conceptual nature of level sets has become apparent. The embedding of the information on the domain and its boundary into a higher-dimensional function has made it easy to extract also additional information as the normal direction and the curvature (see (4.44)) from this higher dimensional function.

Furthermore, singular behaviour in the evolution of the domain such as a splitting of the domain or the merging of two disconnected subdomains is covered in the smooth evolution of the higher dimensional function. In this way, the Level Set Approach is most appropriate if topological changes in the geometry are expected or at least if they cannot be ruled out.

We have experienced that it is extremely simple to implement early, crude Level Set Algorithms such that the general scheme is easily accessible, both from a conceptual and a computational point of view.

The methodology has also proved to be much more versatile than explicit boundary representations in the sense that it allows wider classes of geometric setups. 
We focus here on two particular features. On the one hand the realization of domains with an arbitrary finite number of connected components is easy and can be handled adaptively. On the other hand the restriction to starlike shapes is not present. In particular, the creation of a horseshoe-shaped boundary is completely generic in the context of the Level Set Approach.

The example from Section 5.3 has shown another very strong point of the Level Set Approach. It is this appealing way how separation conditions or forbidden areas are incorporated. The velocity field is simply set to zero in these regions with the effect that the level set function stays constant with respect to time, i.e. the 0-level set stays fixed in such a region or it does not propagate into it if it has not been there initially. We mention here that there exist also other approaches for topology-preserving level set methods in the literature (see [4]).

In the course of the discussion of the numerical scheme of the Steepest Descent Algorithm we have concerned ourselves also with the suppression of oscillatory behaviour of the boundary. We note that there exist various approaches for smoothing the boundary in Level Set Methods, although we have employed none of them in our studies. We pick out two approaches that are representatives for their specific class of methods. The first approach uses the information that is inherent in the level set function. The boundary is smoothed in the evolution by adding a curvature dependent term to the level set equation (4.39). Such a term is diffusive in its nature. Hence, it acts as a counterpart to the formation of small-scale oscillations or corners. The second approach is indirect, as smoothes the evolution of the 0-level set by smoothing the velocity field in each time step. In particular, the velocity field is regularized using the $H^{1}$ Sobolew-norm of the velocity field (see [5]).

Nevertheless, the Level Set Approach does not only have advantages. In the context of the problem we have been studying in this thesis, we have made the observation that implicit boundary representations do not really fit to the boundary-oriented integral equation method which is at the heart of the Boundary Value Problem. So, the ideas presented in Section 4.3 should rather be viewed as a first step towards the development of tools that efficiently and reliably combine boundary integral methods and level set methods. Therefore, we consider the existing drawbacks in detail.

The first drawback is immediately obvious from the Level Set Algorithm 4.18. We need two additional steps, the contouring and the extension, to incorporate the 'boundary' velocity, which we have derived in Chapter 3, into the level set framework.

In these steps even more drawbacks are contained. We have seen in the numerical experiments that the approximation points for the boundary that are obtained by the contouring algorithm are not smoothly spaced. The has a strongly negative effect on the integral equation method with which we solve the Boundary Value Problem and compute the 'boundary' velocity. Consequently, also the extension 
algorithm suffers from this drawback. At the same time the finite difference approximations to the gradient and to the curvature have only low order accuracy which additionally contributes to the effect.

The geometric accuracy of the Level Set Approach is naturally limited by the resolution of the discrete grid, which is two-dimensional. Hence, a fine resolution affects the computation time and the storage requirements much more heavily than a fine discretization of a basically one-dimensional boundary parametrization.

Finally, the 'boundary' velocity that is computed for the Level Set Approach is somehow the opposite extreme to the approximation of the radial function by trigonometric polynomials. The basis functions we have used in (4.42) to define the 'boundary' velocity are more or less a Lagrange basis for the normal field on the 0-level set, for which we know nothing more than the Lagrange property. In this sense, the 'boundary' velocity decouples, as it does not incorporate information from a basis function $\alpha_{j} N$ at the point $x_{i}$, although $\alpha_{j} N$ should influence the value of the 'boundary' velocity via its derivatives. The effect of this is that the approach does not make up for an oscillatory Fréchet derivative. But this has to be taken into account due to drawbacks described above.

Hence, we may summarize that the Level Set Approach displays several appealing and desirable features as a general concept for geometric optimization that let it appear to be superior to an explicit boundary representation. Nevertheless, one has to keep in mind that there also exist various, partly severe, drawbacks. With the tools we have employed in this thesis, these drawbacks still lead to the consequence that implicit boundary representations are not yet a perfect match for boundary integral methods. We will present some additional ideas in the outlook that may help improving the connection between Level Set Methods and boundary integral equations. If the ideas presented there can be successfully incorporated into the general scheme, then the combination of both approaches is expected to render a powerful tool in geometric optimization as it complements the efficiency and the rapid convergence of boundary integral methods with the versatility and conceptual simplicity of Level Set Methods.

We contrast the features of the Level Set Approach in the following by the a corresponding analysis for the Steepest Descent Approach. Here we begin by presenting the drawbacks that exist, and for many of which we have presented strategies in Section 4.2 how these drawbacks can be dealt with adequately.

The central point of the approach via explicit boundary representation is the idea to split the parametrization of the boundary into an angular and a radial part. Then the optimization is carried out with respect to the radial part only, where the admissible set of radial functions is a subset of a finite-dimensional space. This already discloses many drawbacks of the method. Firstly, an admissible radial function has to be positive, while the finite dimensional approximation space naturally allows also negative functions. Hence, special care has to be taken 
within an implementation to ensure the positivity for each iterate. Secondly, the approximation space allows on its own account arbitrary large coefficients for any basis function. This induces additional problems such as separation problems or oscillatory behaviour. Thirdly, the resulting domains are always starlike. Geometries such as horseshoe-shaped domains that may be of particular interest from an applicational point of view cannot be realized in the framework of this approach.

Furthermore, the approximation spaces presented in Section 4.2 have either global or mildly localized support. This leads to more or less global perturbations of the domain, when claculating the Fréchet derivative. The numerical examples from Chapter 5 have shown, that contrarily the model requires local perturbations of the domain. This is understood in the sense that the side of the domain $D$ that is not facing $\Gamma$ only gives a marginal contribution to the behaviour of the functional and the current distribution.

In order to overcome what we have phrased above as separation problems, namely securing $\Gamma \cap D=\emptyset$, we have introduced the Augmented Lagrangian Approach, where we have prescribed a hard constraint on the position of the boundary $\partial D$ for a certain fixed angular direction. The idea of enforcing a separation of $D$ and $\Gamma$ in this way goes further than what is originally required by the problem. It arbitrarily fixes a distance between $D$ and $\Gamma$ for one particular direction leaving all other directions as well as the distance itself out of account. For arbitrarily placed centres the approach is hence not appropriate for ensuring $D \cap \Gamma=\emptyset$.

Finally, we observe from the Steepest Descent Algorithm 4.11 directly that the case of finitely many connected components for $D$ cannot be handled as easily as in the case of the Level Set Approach. Moreover, we see that adaptive strategies cause even more severe problems. This is understood in the sense that the merging of two starlike domains cannot be expressed in the same framework. Similarly, the framework itself contains no mechanism that indicates when a splitting for a domain should take place. Hence, the Steepest Descent Approach is topologically stiff, with the number of connected components for $D$ being an input parameter that is given a priori and which stays fixed throughout the algorithm.

Some of the drawbacks discussed above have been addressed in the course of the Steepest Descent Approach, and we have been able to suggest regularization terms to tackle these problems at least for the situations that are relevant from an applicational perspective. Other deficiencies like the topological stiffness are an inherent property of the approach via explicit boundary representations itself and hence cannot be resolved in this framework.

The drawbacks mentioned above are contrasted by advantages of the approach via explicit boundary representations, which turn out to be of decisive quality. The most important point is that the Steepest Descent Approach is the perfect match for the boundary integral equation method for the Boundary Value Problem. The sensitivity analysis as well as the Steepest Descent Approach are for- 
mulated in exactly the same language as the boundary integral equation method. Hence, the evaluation of the Fréchet derivative is achieved without much further work. Moreover, it has a natural interpretation for updating the boundary of $D$. This leads to an appealing simple structure of the basic iterative procedure and makes it extremely simple to use.

As a second argument we adduce that it is conceptually easy to incorporate schemes which either suppress oscillatory behaviour in the boundary, or which are employed for securing $\Gamma \cap D=\emptyset$. This can be seen from the regularization terms $G_{c}$ and $G_{\vartheta, d}$ from Section 4.2. Although we have argued above that the Augmented Lagrangian Approach is not effective when viewed as a general concept, we point out that the regularization term $G_{\vartheta, d}$ works extremely efficiently in the cases that are relevant from an applicational point of view. The regularization term $G_{c}$ that suppresses oscillations in the radial function serves several purposes at the same time. Firstly, it can be viewed as a design parameter that influences the cavity which the domain is forming around the arc. Secondly, it leads to essentially non-oscillatory boundary curves in regions that are not facing the arc. And thirdly, it can also be regarded as a stability parameter as it helps ensuring the positivity of the radial function. These advantages of the Steepest Descent Approach are complemented by a rapid convergence of the boundary integral equation method as we have smooth boundary representations which allow comparatively coarse discretizations of the boundary.

We conclude that, as far as this thesis is concerned, the approach via explicit boundary representations is better suited for the Geometric Optimization Problem than the Level Set Approach. From the side of the Steepest Descent Approach the reason is that we have been able to introduce regularization terms that deal efficiently and satisfactorily with the most immediate drawbacks for the situations which are considered the most relevant from an applicational perspective. From the point of view of Level Set Methods the reason is the desired versatility and adaptivity that we have gained with the Level Set Approach does not play an important role in the problem at hand. In particular we have never experienced the tendency in the geometric evolution for a merging or splitting to take place. It is our personal view that this is due to the local nature of the homogenization process. Merging can only occur if the two domains being situated at the opposing endpoints of the arc evolve across the entire length of the arc. This would require a stronger dependence of the domain boundary on the current distribution on the other endpoint of the arc - a behaviour which is not supported by the examples of the previous chapter. As the crucial advantages of the Level Set Approach have turned out to be of minor importance for the problem at hand, the approach is in an inferior position to the Steepest Descent Approach, as its drawbacks gain weight. Nevertheless the approach via implicit boundary representations has proved to be a powerful alternative provided the versatility and adaptivity play a decisive role in an optimization problem. 


\subsection{Outlook}

We end our considerations of the shape optimization problem in superconductivity by giving ideas how the work compiled in this thesis can be dwelled upon.

Let us first of all mention that the work at hand is by no means meant to be complete or comprehensive. In nearly every part of this thesis there are possibilities to extend or modify the presented material.

As a first issue we consider the mathematical model that leads to the Boundary Value Problem from Chapter 2. The experiments from Chapter 5 have shown that the optimized geometries of the magnetic environment for a single superconducting film can reduce the peak in the current distribution by a factor of up to ten. This is a strong result on its own account as it allows roughly speaking up to ten times stronger currents to be carried in a superconducting film that is magnetically shielded by magnets as compared to the same film without shielding. On the other hand the total currents that are transported are still comparatively small such that the result on its own does not make superconductivity competitive. Nevertheless, by combining several superconducting films one might be able to attain strengths for the total current that are interesting for applications.

In this context one can pose the question whether the fields that are generated by the currents in these films may not themselves serve as shieldings for the other films. For the mathematical model this leads to the study of the interactions of several disjoint open arcs, in combination with additional permanent magnets. In such a problem also an appropriate distribution of the total current to each film becomes a relevant question as it may heavily influence the result.

Turning back to the setup from this thesis, we have to mention that the characterization of the current distribution from Theorem 2.15 is not completely satisfactory. We have been able to prove in Section 2.4 that the current distribution for the arc $\Gamma$ either vanishes at the endpoints of $\Gamma$ or develops singularities of at most square root type there. Contrary to this result, there is no occasion in our experience where either a singularity of lower order or even a non-singular current distribution has occured. This experience is coherent with the result of Hayashi [34], where it was proven that the solution to a Dirichlet problem for the Helmholtz equation in the exterior of an open arc always develops square root singularities. Hence we propose

Conjecture 6.1. Let $(\Gamma, D)$ be an admissible geometry, and let $I \neq 0$. Then the current distribution for $\Gamma$ is always singular at the endpoints $z_{ \pm 1}$ of $\Gamma$, and the singularity is of square root type. In other words, we have

$$
\left[\frac{\partial u}{\partial \nu_{\Gamma}}\right](x)=\left(\left|x-z_{ \pm 1}\right|\right)^{-\frac{1}{2}} f_{ \pm 1}(x), \quad \Gamma \ni x \rightarrow z_{ \pm 1},
$$

where the functions $f_{ \pm 1}$ are bounded in neighbourhoods of $z_{ \pm 1}$, and are different from zero at $z_{ \pm 1}$. 
Strongly related to a comlete characterization of the current distribution are modifications that might be applied to the Geometric Optimization Problem in general. In Lemma 1.15 we have seen how delicate the behaviour of functions in $C^{*}(\Gamma)$ is with respect to the endpoints. The weight function in the objective functional (3.4) complements this behaviour. In this thesis, we have made a particular choice (3.5) for the weight function, that was motivated from the simplicity that the functional thereby obtains, but it is by no means clear whether this choice is optimal. Further studies in this direction seem appropriate as the weight function should not disguise prominent features of the current distribution.

Turning to the approximation schemes for the Geometric Optimization Problem we find several starting points for possible extensions.

So, the choices for the finite-dimensional approximation spaces used in the Steepest Descent Approach have been either basic or extremely design specific. It may be well worth studying other approximation spaces that have either compact support or at least a localized support with strong single peaks. The aim should be to obtain smaller opening angles for the cavity of the domain $D$ around $\Gamma$ in combination with an essentially non-oscillatory boundary curve in all other regions of the boundary of $D$. We have to add that such approaches have to be carried out rather cautiously, because the approximation scheme for the Boundary Value Problem requires balancing the distance between the boundary curves and the level of discretization for each boundary.

A second issue within the framework of the Steepest Descent Approach, which can lead to improvements, is to think about alternatives to the Augmented Lagrangian Approach. We have already pointed out in Section 4.2, where the weak points of the Augmented Lagrangian Approach are. As a possible remedy we have suggested penalization strategies with a penalty term of the form of (4.31). We judge further investigations in that direction as rather promising, in particular as one might be able to exploit the insights gained there for a more localized approach for curvature regularization. The basic rule here could be the following.

Whenever $\partial D$ and $\Gamma$ are close, we want to punish oscillations less, but penalize the too small distance heavier. In areas where the distance between $\partial D$ and $\Gamma$ is large we do not want to penalize the distance, but want to suppress oscillations comparatively strongly.

Nevertheless, it will be quite tricky to come up with a penalty term which realizes the above defined goal satisfactorily.

The third point we would like to address here, is the development of a more sophisticated line search. In cases where the evaluation of the Fréchet derivative is much more costly than solving an additional Boundary Value Problem, it is desirable and worth the effort to determine an optimal step size for the Steepest Descent Algorithm. We have used the line search only in a rather crude way in Section 4.2. On the one hand we have used the approach for regularization by halving step size until the positivity of the radial function is restored, on the other 
hand we have suggested to sample over a fixed set of step sizes to determine the optimal step size out of these choices. We think that the use of Wolfe's condition will lead to improved performance of the line search.

The Level Set Approach still leaves the most room for improvements. We have seen that although the approach is conceptually simple and straightforward, the numerical results can not compete with the results obtained by the Steepest Descent Algorithm. The possible reasons for this have been discussed in detail in the first part of this chapter. We point out that up to the present there is no agreed way how to combine the two conflicting approaches of either explicit or implicit boundary representation efficiently and satisfactorily. Consequently, two different paths become apparent how to proceed.

A first idea would be to exploit the geometric information contained in the discrete version of the level set function even more. This would mean to employ spatial and temporal discretizations of higher accuracy to handle the rapidly changing velocity field more adequately. Based on these more accurate information about the shape of the 0-level set, one can also think of interpolating additional boundary points in order to obtain an approximately equidistantly spaced representation of the 0-level set.

A second idea is of a more conservative nature and follows the approach of Ferrayé et. al (see [26, 27, 28, 29]). It restricts the information extracted from the level set function to the position of the 0-level set using the extrapolation as in Section 4.3. All other required quantities are then obtained after a second approximation step. In this step we use tools from approximation theory to generate a smooth parametrized curve which approximates the 0-level set based on the discrete information we have from the first step.

Finally, also the context in which we view the physical application can be modified and extended. On the one hand, a generalization to the three-dimensional case seems interesting. Here, one is not so much interested in transport currents, but in so-called magnetization experiments, where an externally generated magnetic field induces a circular shielding current in the superconducting medium. In this context one might seek to extend the mathematical model to the case of twodimensional planar cracks, and develop an analogous analysis as has been done here. On the other hand, one might want to view the existing two-dimensional problem in the context of alternating currents instead of direct currents. This would then lead to time-harmonic magnetic fields, which can be modelled using the Helmholtz equation. Here, an ansatz similar to the one presented in this thesis seems possible, both for the Boundary Value Problem and for the Geometric Optimization Problem. 


\section{Bibliography}

[1] D. Adalsteinsson, J. A. Sethian: A fast level set method for propagating interfaces, J. Comp. Phys. 118, 269 - 277, (1995)

[2] D. Adalsteinsson, J. A. Sethian: A unified level set approach to etching, deposition and lithography I: Algorithms and two-dimensional simulations, J. Comp. Phys. 120, 128 - 144, (1995)

[3] D. Adalsteinsson, J. A. Sethian: The fast construction of extension velocities in level set methods, J. Comp. Phys. 148, 2 - 22, (1999)

[4] O. Alexandrov, F. Santosa: A topology-preserving level set method for shape optimization, J. Comp. Phys. 204, 121 - 130, (2005)

[5] G. Allaire, F. Jouve, A.-M. Toader: Structural optimization using sensitivity analysis and level set methods, J. Comp. Phys. 194, 363 - 393, (2004)

[6] H. Ben Ameur, M. Burger, B. Hackl: Level set methods for geometric inverse problems in linear elasticity, Inv. Probl. 20, 673 - 696, (2004)

[7] M. S. Berger: Nonlinearity and Functional Analysis, Academic Press, New York, 1977

[8] G. Blatter, M. V. Feigelman, V. B. Geshkenbein, A. L. Larkin, V. M. Vinokur: Vortices in high-temperature superconductors, Rev. Mod. Phys. 66, 1125, (1994).

[9] M. Bochniak, F. Cakoni: Domain sensitivity analysis of the acoustic far-field pattern, Math. Meth. Appl. Sci., 25, 595 - 613, (2002)

[10] V. Born, K. Guth, H. C. Freyhardt, Ch. Jooss, Self-enhanced flux penetration into small-angle grain boundaries in $\mathrm{YBaCuO}$ films, Supercond. Sci. Technol. 17, 380 - 385, (2004)

[11] W. Buckel: Supraleitung: Grundlagen und Anwendungen, 5. Aufl., VCH, 1994 
[12] M. Burger: A framework for the construction of level set methods for shape optimization and reconstruction, Interf. Free Bound. 5, 301 - 330, (2003)

[13] M. Burger: A level set method for inverse problems, Inv. Probl. 17, 1327 $1355,(2001)$

[14] T. F. Chan, X.-C. Tai: Level set and total variation regularization for elliptic inverse problems with discontinuous coefficients, J. Comp. Phys. 193, 40 $66,(2004)$

[15] S. Chen, B. Merriman, S. J. Osher, P. Smereka: A simple level set method for solving Stefan problems J. Comp. Phys. 135, 8 - 29, (1997)

[16] L. T. Cheng, R. P. Fedkiw, F. Gibou, M. Kang: A Symmetric Method for Implicit Time Discretization for the Stefan Problem CAM Report 00-07, University of California, Los Angeles, 2000

[17] E. T. Chung, T. F. Chan, X.-C. Tai: Electrical impedance tomography using level set representation and total variational regularization, J. Comp. Phys. 205, $357-372,(2005)$

[18] D. Colton, R. Kress: Inverse Acoustic and Electromagnetic Scattering Theory, 2nd ed., Springer, Berlin, 1998

[19] R. Courant, D. Hilbert: Methoden der mathematischen Physik, 2. Aufl., Springer-Verlag, Berlin, 1968

[20] M. G. Crandall, L. C. Evans, P.-L. Lions: Some Properties of Viscosity Solutions of Hamilton-Jacobi Equations, Trans. Amer. Math. Soc. 282, 487 - 502, (1984)

[21] M. G. Crandall, P.-L. Lions: Viscosity Solutions of Hamilton-Jacobi Equations, Trans. Amer. Math. Soc. 277, 1 - 42, (1983)

[22] M. C. Delfour, J.-P. Zolesio: Shapes and Geometries, SIAM Advances in Design and Control, Philadelphia, 2001

[23] P. Deuflhard, A. Hohmann: Numerische Mathematik I, 3. Aufl., Walter de Gruyter, Berlin, 2002

[24] K. Eriksson, D. Estep, P. Hansbo, C. Johnson: Computational Differential Equation, Cambridge University Press, Cambridge, 1996

[25] L. C. Evans: Partial Differential Equations, Amer. Math. Soc., Providence, 1998 
[26] R. Ferrayé, J.-Y. Dauvignac, C. Pichot: A boundary-oriented inverse scattering method based on contour deformations by means of a level set method for radar imaging, Int. J. Appl. Electrom. Mech. 15, 213 - 218, (2002)

[27] R. Ferrayé, J.-Y. Dauvignac, C. Pichot: An accurate boundary-oriented method for transverse magnetic scattering by perfectly conducting and/or dielectric cylinders of arbitrary cross-section, Conference on Antennas and Propagation AP2000 (Davos, Switzerland), Abstracts, Vol. 2, 332; Full papers, CD-ROM, paper p0248.pdf (4 pages), (2000).

[28] R. Ferrayé, J.-Y. Dauvignac, C. Pichot: An inverse scattering method based on contour deformations by means of a level set method using frequency hopping technique, IEEE Transact. Antennas Prop. 51 (5), 1100 - 1113, (2003)

[29] R. Ferrayé, J.-Y. Dauvignac, C. Pichot: Reconstruction of complex and multiple shape object contours using a level set method J. Electrom. Waves Appl. 17 (2), 153 - 181, (2003)

[30] Yu. A. Genenko, A. Snezhko, H. C. Freyhardt: Overcritical states of a superconductor strip in a magnetic environment, Phys. Rev. B 62, 3453 - 3472, (2000)

[31] D. Gilbarg, N. S. Trudinger: Elliptic Partial Differential Equations of Second Order, 2nd ed., Springer, Berlin, 1998

[32] M. Gyure, C. Ratsch, B. Merriman, R. E. Caflisch, S. J. Osher, J. Zinck, D. Vvedensky: Level set methods for the simulation of epitaxial phenomena, Phys. Rev. E 59, R6927, (1998)

[33] J. Haslinger, R. A. E. Mäkinen: Introduction to Shape Optimization, SIAM Advances in Design and Control, Philadelphia, 2003

[34] Y. Hayashi: The Dirichlet Problem for the Two-Dimensional Helmholtz Equation for an Open Boundary, J. Math. Anal. Appl., 44, 489 - 530, (1973)

[35] H. Heese: An integral equation method for a boundary value problem in superconductivity, Math. Meth. Appl. Sci. 27, 1725 - 1743, (2004)

[36] H. Heese: Integralgleichungsmethode für ein Randwertproblem aus der Theorie der Supraleitung, Diplomarbeit, NAM, 2003

[37] F. Hettlich: Fréchet derivatives in inverse obstacle scattering, Inv. Prob. 11, $371-382,(1995)$

[38] F. Hettlich: Erratum: Fréchet derivatives in inverse obstacle scattering, Inv. Prob. 14, 209 - 210, (1998) 
[39] T. Hohage: Iterative methods in inverse obstacle scattering: Regularization theory and nonlinear exponentially ill-posed problems, Universitätsverlag Rudolf Trauner, Linz, 1999

[40] J. D. Jackson: Klassische Elektrodynamik, 3. Aufl., De Gruyter, Berlin 2002

[41] H. Jarzina, Ch. Jooss, H. C. Freyhardt: Overcritical Meissner current densities in $\mathrm{YBaCuO}$ thin films in soft magnetic environments, J. Appl. Phys. 91, 3775, (2002)

[42] J. Jost: Postmodern Analysis, 2nd ed., Springer, Berlin, 2003

[43] Ch. Jooss, E. Brinkmeier, H. Heese: Combined experimental and theoretical study of field and current conditioning in magnetically shielded superconducting films, Phys. Rev. B 72, 144516, (2005)

[44] A. Kirsch: The domain derivative and two applications in inverse scattering theory, Inv. Prob. 9, 81 - 96, (1993)

[45] R. Kress: Ein ableitungsfreies Restglied für die trigonometrische Interpolation periodischer analytischer Funktionen, Numer. Math. 16, 389 - 396, (1971)

[46] R. Kress: Inverse scattering from an open arc, Math. Meth. Appl Sci. 18, $267-293,(1995)$

[47] R. Kress: Linear Integral Equations, 2nd ed., Springer, New York, 1999

[48] O. A. Ladyzhenskaya: The Boundary Value Problem of Mathematical Physics, Springer, New York, 1985

[49] S. Langer: Integralgleichungsmethode für das Neumann Problem in einem Gebiet mit Schlitz, Diplomarbeit, NAM, 2003

[50] S. G. Mikhlin: Mathematical Physics, an Advanced Course, North-Holland, Amsterdam, 1970

[51] L. Mönch: On the numerical solution of the direct scattering problem for an open sound-hard arc, J. Comp. Appl. Math., 71, 343 - 356, (1996)

[52] H. Multhopp: Die Berechnung der Auftriebsverteilung von Tragflügeln, Luftfahrt-Forschung 4, 153 - 169 (1938)

[53] N. I. Muskelishvili: Singular Integral Equations, Noordhoff, Groningen, 1953

[54] S. J. Osher, R. P. Fedkiw: Level Set Methods and Dynamic Implicit Surfaces, Springer-Verlag, New York, 2003 
[55] S. J. Osher, F. Santosa: Level set methods for optimization problems involving geometry and constraints I. Frequencies of a two-density inhomogeneous drum, J. Comp. Phys. 171, 272 - 288, (2001)

[56] S. J. Osher, J. A. Sethian: Fronts propagating with curvature-dependent speed: algorithms based on Hamilton-Jacobi formulations, J. Comp. Phys. 79, 12 - 49, (1988)

[57] O. Pironneau: Optimal Shape Desgin for Elliptic Systems, Springer-Verlag, New York, 1984

[58] R. Potthast: Fréchet differentiability of boundary integral operators in inverse acoustic scattering, Inv. Prob. 10, 431 - 447, (1994)

[59] S. Prössdorf, B. Silbermann: Numerical Analysis for Integral and Related Operators, Akademie-Verlag, Berlin 1991, and Birkhäuser-Verlag, Basel 1991

[60] A. Roger: Reciprocity theorem applied to the computation of functional derivatives of the scattering matrix, Electromagn. 2, 69 - 83, (1982)

[61] E. Rouy, A. Tourin: A viscosity solutions approach to shape-from-shading, SIAM J. Num. Anal. 29, 867 - 884, (1992)

[62] L. Rudin, S. J. Osher, E. Fatemi: Nonlinear total variation based noise removal algorithms, Physica D 60, 259 - 268, (1992)

[63] F. Santosa: A level set approach for inverse problems involving obstacles, ESAIM Contr. Optim. 1, 17 - 33, (1996)

[64] J. A. Sethian: A fast marching level set method for monotonically advancing fronts, Proc. Nat. Acad. Sci. 93(4), 1591 - 1595, (1996)

[65] J. A. Sethian: An Analysis of Flame Propagation, Ph.D. Thesis, Universit of California, Berkeley, 1982

[66] J. A. Sethian: Levet Set Methods and Fast Marching Methods, 2nd ed., Cambridge University Press, Cambridge, 1999

[67] J. A. Sethian, J. Strain: Chrystal growth and dendritic solidification, J. Comp. Phys. 98, 231 - 253 (1992)

[68] J. A. Sethian, A. Wiegmann: Structural Boundary Design via Level Set and Immersed Interface Maethods, J. Comp. Phys. 163, 489 - 528, (2000)

[69] J. Simon: Differentiation with respect to the domain in boundary value problems, Numer. Funct. Anal. Optim. 2, 649 - 687, (1980) 
[70] J. Sokolowski, J.-P. Zolesio: Introduction to Shape Optimization, SpringerVerlag, Berlin, 1992

[71] M. Sussman, P. Smereka, S. J. Osher: A level set approach for computing solutions to incompressible two-phase flow, J. Comp. Phys. 114, 146 - 159, (1994)

[72] X.-C. Tai, T. F. Chan: A survey on multiple level set methods with applications for identifying piecewise constant functions, Int. J. Num. Anal. Mod. $\mathbf{1}(1), 25-47,(2004)$

[73] J. Tsitsiklis: Efficient Algorithms for globally optimal trajectories, IEE Trans. Autom. Contr. 40, 1528 - 1538, (1995)

[74] S. S. Vinogradov, P. D. Smith, E. D. Vinogradova: Canonical Problems in Scattering and Potential Theory PartI: Canonical Structures in Potential Theory, Chapman \& Hall / CRC, Boca Raton, 2001

[75] J. Werner: Optimization. Theory and Applications, Vieweg, Braunschweig, 1984

[76] Y. Yan, I. H. Sloan: On integral equations of the first-kind with logarithmic kernels, J. Int. Eq. Appl. 1, 549579, (1988)

[77] H.-K. Zhao, T. Chan, B. Merriman, S. J. Osher: A variational level set approach to multiphase motion, J. Comp. Phys. 127, 179 - 195, (1996) 


\section{Curriculum vitae - Lebenslauf}

$\begin{array}{ll}\text { Name: } & \text { Harald Heese } \\ \text { geboren: } & \text { 1. August } 1977 \\ \text { Geburtsort: } & \text { Kassel } \\ \text { Familienstand: } & \text { ledig } \\ \text { Staatsangehörigkeit } & \text { deutsch } \\ \text { Wohnsitz: } & \text { Göttingen }\end{array}$

\section{Akademische Ausbildung und Tätigkeiten}

$06 / 97$

$10 / 98-07 / 03$

$07 / 03$

seit $08 / 03$

06/2006
Abitur am Engelsburggymnasium Kassel

Studium der Mathematik an der Georg-August-Universität Göttingen

Diplomprüfung an der Mathematischen Fakultät der Georg-August-Universität Göttingen

Wissenschaftlicher Mitarbeiter am Institut für NAM der Georg-August-Universität Göttingen und Promotionsstudium an der Fakultät

Einreichung der Dissertation 